\title{
FORMULAS AND TABLES FOR THE CALCULATION OF MUTUAL AND SELF-INDUCTANCE
}

\author{
By Edward B. Rosa and Frederick W. Grover
}

\section{CONTENTS}

(See also Index)

INTRODUCTION. .

I. MUTual, Inductance of Two Coaxial Circles:

(a) Formulas-

I, 2, Maxwell's formulas in elliptic integrals............

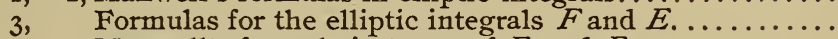

Maxwell's formula in terms of $F_{0}$ and $E_{0} \ldots \ldots \ldots \ldots \ldots$

Maxwell's formula expressed as a $k$ series.............

Maxwell's formula expressed as series in $k_{1} \ldots \ldots \ldots \ldots$

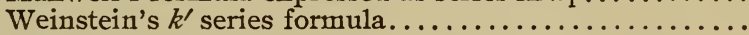

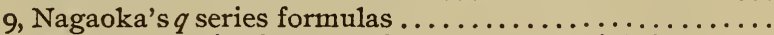

Io, II, Maxwell's series formulas for any two coaxial circles....

I2, Maxwell's series formula for circles of equal radii.......

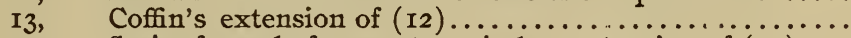

I4, Series formula for any two circles, extension of (Io)....

I5, Formula for coplanar circles, derived from (r4).......

I6, Havelock's formula (extended) for circles near together.

I7, Havelock's formula, for circles far apart.............

I8, Mathy's formula (corrected)....................

I9, Simple form of Mathy's formula for the case $r_{1}^{2}=2 r_{2}^{2} \ldots$

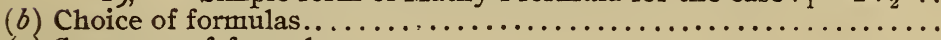

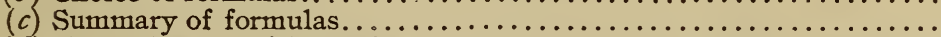

(d) Examples I-I9, illustrating the formulas for the mutual inductance

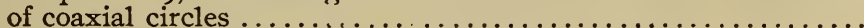

2. Mutual Inductance of TWo Coaxial CoIIs:

(a) Formulas-

2O, Rowland's formula, equal radii, but unequal section....

2I, Rowland's formula, equal radii and equal section .......

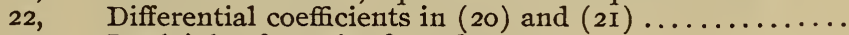

23, Rayleigh's formula of quadratures..................

24, Same for coils of equal radii and equal section ...........

25,26 , Formulas for errors of formulas $(2 \mathrm{I})$ and $(24) \ldots \ldots \ldots 36,37$

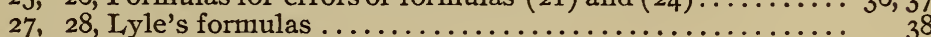

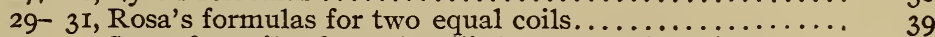

32, Same for coils of equal radii, but unequal sections....... 39

33, The Rosa-Weinstein formula.................. 40

34, Formula in terms of self-inductance ................ 4 I

35, Geometric mean-distance formula ................. 42

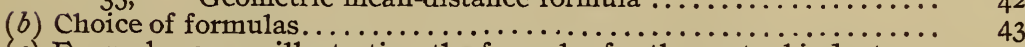

(c) Examples $20-33$, illustrating the formulas for the mutual inductance

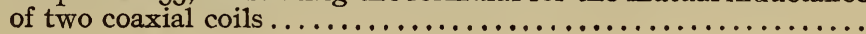

.

9

2
3




\section{Mutual Inductance of Coaxial Solenoids:}

(a) Formulas--

36, 37, Maxwell's formulas for two concentric solenoids of

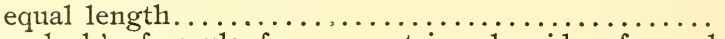

Havelock's formula for concentric solenoids of equal

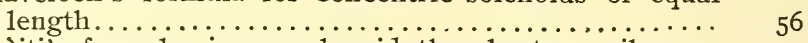

Ròiti's formula, inner solenoid the shorter, coils con-

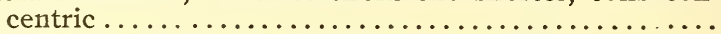

40, Gray's formula, coaxial coils not concentric.............

4I, Formula for the Gray electrodynamometer..............

42, Searle and Airey's formula, inner coil the shorter, coils

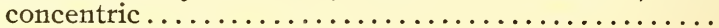

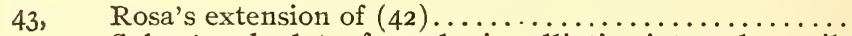

44, Cohen's absolute formula in elliptic integrals, coils

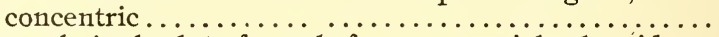

45, Nagaoka's absolute formula for any coaxial solenoids. .

46,47 , Russell's formulas ...................... 67,68

(b) Mutual inductance of a short secondary, on the outside of a long

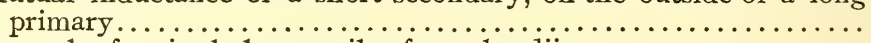

(c) Formulas for single layer coils of equal radii-

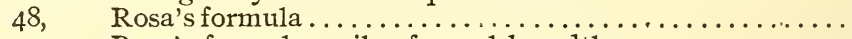

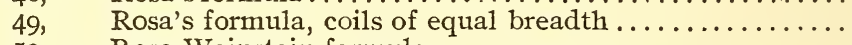

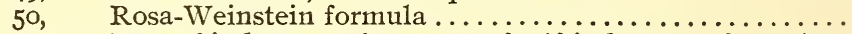

$5 \mathrm{I}, 52$, Mutual inductance by means of self-inductance formulas.

(d) Other formulas and Olshausen's absolute formula ..............

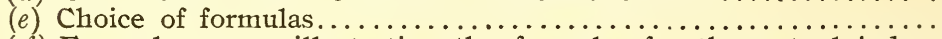

$(f)$ Examples $34-47$, illustrating the formulas for the mutual induc-

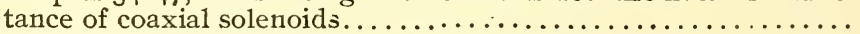

4. Mutual, Inductance of a Circle and a Coaxial, Single-Iayer CoIl:

(a) Formulas-

53, Lorenz's series formula ................... 98

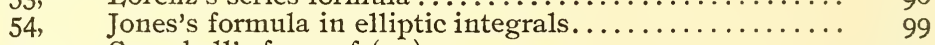

$55, \quad$ Campbel1's form of $(54) \ldots \ldots \ldots \ldots \ldots \ldots \ldots \ldots \ldots \ldots \ldots \ldots \ldots \ldots$ IOO

56,57 , Rosa's series formulas ................................

(b) Examples, 48-5I, illustrating the formulas for the mutual inductance of a circle and a coaxial single-layer coil ..............

5. The Seiff-Inductance of a Circular Ring of Circular Section:

(a) Formulas-

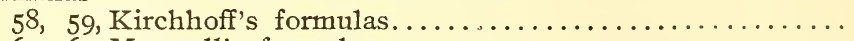

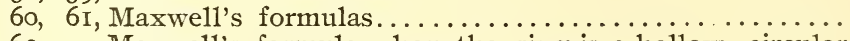

62, Maxwell's formula when the ring is a hollow, circular

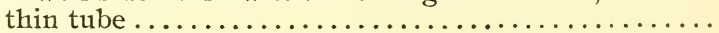

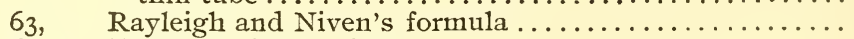

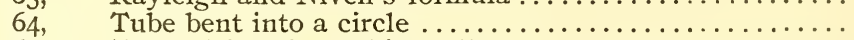

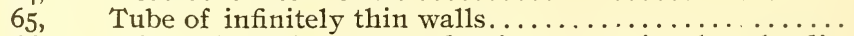

66, Wien's formula, current density proportional to the dis-

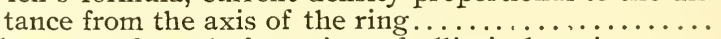

67 , Thomson's formula for a ring of elliptical section......

(b) Examples 52-56, illustrating the formulas for the self inductance of

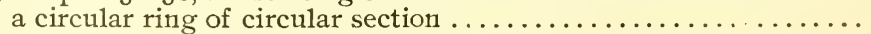

6. THE SELF-INDUCTANCE OF A SINGLE IAYER COIL OR SOLENOID:

(a) Formulas-

68, Formula for infinitely long solenoid ........... II6

69,70, Rayleigh and Niven's formulas ...................... II I 7

$7 \mathrm{r}, \quad$ Coffin's series formula, extension of $(69) \ldots \ldots \ldots \ldots \ldots \ldots \ldots \ldots \ldots \ldots \ldots$ I 7

72,73 , Lorenz's absolute formula in elliptic integrals......... II8

74 , Formula for use with Table IV, derived from $(73) \ldots \ldots$ in

$75-78$, Nagaoka's $q$ series formulas .................... II9-I2I

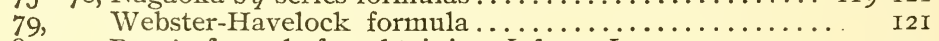

So, $\quad$ Rosa's formula for obtaining $L$ from $L_{s} \ldots \ldots \ldots \ldots \ldots \ldots$ I22

8I, Summation formula........................ I23 
6. The Self-INDUCTANCE OF a Single LAyer CoIl or SolenNoId-Continued.

(a) Formulas-Continued.

83, Formula for $L_{s}$ of a toroidal coil of rectangular section.

84, Rosa's formula for obtaining $L$ from $L_{s}$ for a toroidal

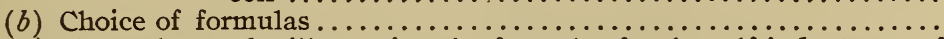

(c) Examples 57-63, illustrating the formulas for the self-inductance of

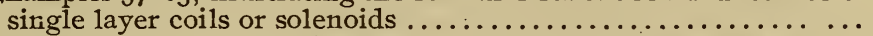

7. The SelfF-INDUCTance of a Circular Corl of Rectangular SeCTION:

(a) Formulas-

85,86, Maxwell's approximate formulas.............. I35, I36

87 , Perry's approximate formula................... I36

88, 89, Weinstein's formulas for $L_{u} \ldots \ldots \ldots \ldots \ldots \ldots \ldots \ldots \ldots$ I37

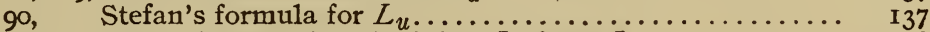

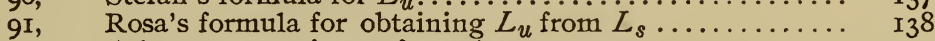

92, Cohen's approximate formula................. I40

93, Maxwell's formula (revised) for obtaining $L$ from $L_{u}$. . I40

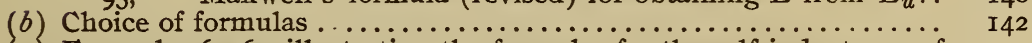

(c) Examples $64-69$, illustrating the formulas for the self-inductance of

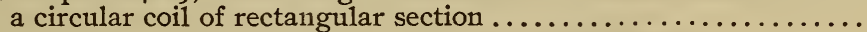

8. SELF AND MUTUAL INDUCTANCE OF IINEAR CONDUCTORS:

(a) Formulas -

94- 97, Self-inductance of a straight cylindrical wire....... I50, I5I

98, 99, Mutual inductance of two parallel wires .............. I5I

IOO, IOI, Self-inductance of a return circuit of two parallel con-

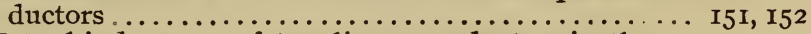

I02, Mutual inductance of two linear conductors in the same straight line .......................... ${ }_{152}$

I03, I04, Self-inductance of a straight rectangular bar .......... I52, I53

I05, I06, Self-inductance of a square, circular section.......... ${ }_{54}$

I07, Self-inductance of a rectangle, circular section ........... I55

Io8, Self-inductance of a rectangle, rectangular section ......

Iog, I Io, Self-inductance of a square, rectangular section....... I55

III, II2, Mutual inductance of two equal, parallel rectangles or

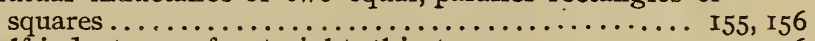

II $3, I_{4}$, Self-inductance of a straight, thin tape ........... I56

II5, Mutual inductance of parallel tapes in the same plane .. $\quad$ I57

II6, Self-inductance of return circuit of two parallel tapes in

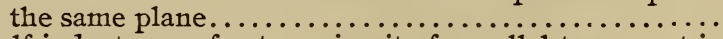

II 7, II8, Self-inductance of return circuit of parallel tapes, not in

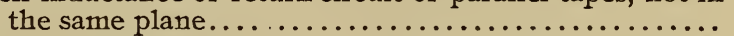

II9, I20, Self-inductance of a return circuit of two concentric conductors............................. 158, 159

I2I, I22, Self-inductance of multiple conductors ............. I59

(b) Examples 7o-8I, illustrating the formulas for the self and mutual

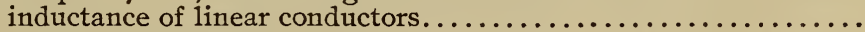

9. FORMULAS FOR GEOMETRICAI AND ARITHMETICAL MEAN DISTANCES:

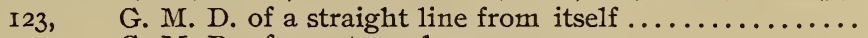

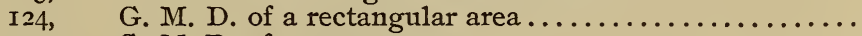

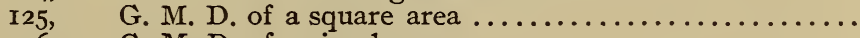

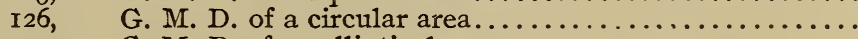

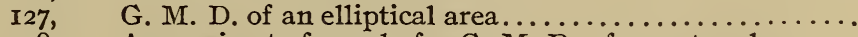

I28, Approximate formula for G. M. D. of a rectangle.....

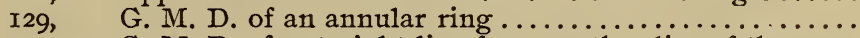

I30, I3I, G. M. D. of a straight line from another line of the same

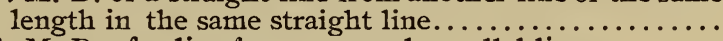

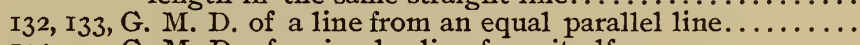

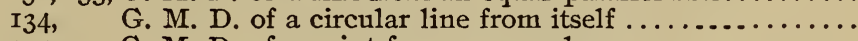

I35, G. M. D. of a point from an annular area ............... 
9. FORMULAS FOR GEOMETRICAI, AND ARITHMETTCAL, MEAN DISTANCESContinued.

I36, G. M. D. of two circular areas from one another........

I37,

I 38 ,

A. M. D. of a line from itself.

I39, I40, A. M. D. and A. M. S. D. of a circular line from itself .

I4I, A. M. D. of an external point from the circumference of a circle

I42, A. M. D. of an external point from a circular area......

IO. HIGH-FREQUENCY FORMULAS AND EXAMPLES:

(a) Formulas-

I43, I44, Kelvin's formula for the resistance and inductance of straight cylindrical wires $\ldots \ldots \ldots \ldots \ldots \ldots \ldots \ldots \ldots$

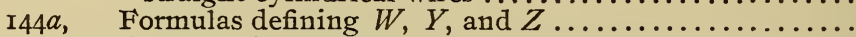

I45-I47, Formulas for the absolute and fractional change of inductance of straight wires with frequency............

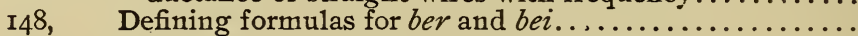

149, Formulas for $W, Y$, and $Z$, small argument...........

150, Savidge's asymptotic formulas for $W, Y$, and $Z \ldots \ldots$.

r5I, Rayleigh's formulas for straight wires, low frequency..

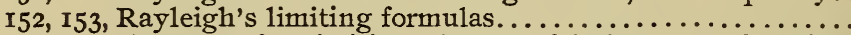

154, 155, Formulas for limiting change of inductance of straight

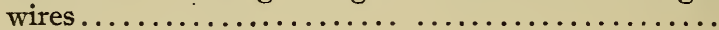

156, Formula for the argument $x \ldots \ldots \ldots \ldots \ldots \ldots \ldots \ldots \ldots \ldots \ldots \ldots \ldots$ I57-r6o, Resistance and inductance of return circuit of two parallel wires . . . . . . . . . . . . . . . . . . . .

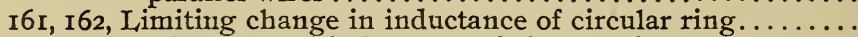
163-166, Resistance and inductance of circular ring ............

(b) Examples 82-85, illustrating the formulas for inductance and re-

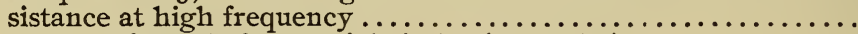

NOTE: Recent formula for coaxial circles (Nagaoka)..............

\section{APPENDIX}

TABLES OF CONSTANTS AND FUNCTIONS USEFUL, IN THE CALCULATION OF MUTUAL, AND SELF-INDUCTANCE

I. Maxwell's table of $\frac{M}{4 \pi \sqrt{A a}}=\left[\left(\frac{2}{k}-k\right) F-\frac{2}{k} E\right]$, for formula (I)..

II. $\log F$ and $\log E$ as functions of $\tan \gamma \ldots \ldots \ldots \ldots \ldots \ldots \ldots \ldots$

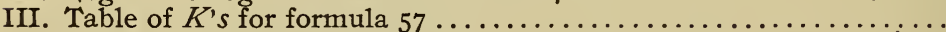

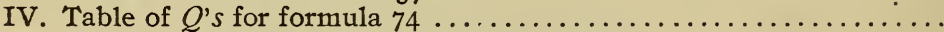

V. Table of constants $A$ and $B$ for formula $82 \ldots \ldots \ldots \ldots \ldots \ldots$

VI. Table of constants $y_{1}$ and $y_{2}$ for formula $90 \ldots \ldots \ldots \ldots \ldots \ldots \ldots$

VII. Table of constants $A$ for formula $80 \ldots \ldots \ldots \ldots \ldots \ldots \ldots \ldots$

VIII. Table of constants $B$ for formula $80 \ldots \ldots \ldots \ldots \ldots \ldots \ldots \ldots \ldots \ldots$

IX. Table of constants $A_{s}$ for formula $91 . \ldots \ldots \ldots \ldots \ldots \ldots \ldots \ldots \ldots$

X. Table of constants $B_{s}$ for formula $9 \mathrm{I} \ldots \ldots \ldots \ldots \ldots \ldots \ldots \ldots \ldots \ldots \ldots$

XI. Table of Napierian Logarithms for numbers $\mathrm{r}-\mathrm{IOO} \ldots \ldots \ldots \ldots \ldots$

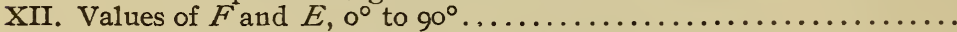

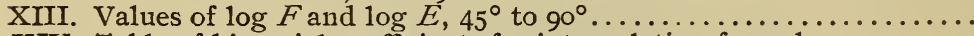

XIV. Table of binomial coefficients for interpolation formula ..........

$\mathrm{XV}$. Values of $q, q_{1}$, and $\epsilon$ for use in formulas $8,9,45,76,77$, and $78 \ldots$

XVI. Values of $\epsilon_{1}$ and $\epsilon_{1}^{\prime}$ as function of $q_{1}$, for use with formulas 6 and $6 a$.

XVII. Coefficients in hypergeometric series of formula $18 . \ldots \ldots \ldots \ldots \ldots$.

XVIII. Location and magnitude of maxima and minima of coefficients in Gray's and Searle and Airey's formulas ..................................

XIX. Values of coefficients in Gray's and Searle and Airey's formulas 40,

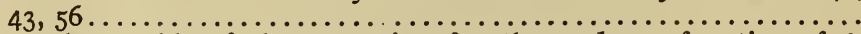

$\mathrm{XX}$. Nagaoka's table of the correction for the ends, as function of $\theta$,

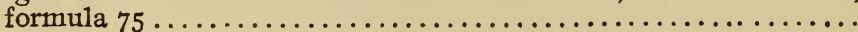


APPENDIX-Continued

TABLES OF CONSTANTS AND FUNCTIONS, ETC.-continued.

XXI. Nagaoka's table of the correction for the ends as function of diameter

length

Page

XXII. Values of $\frac{W}{Y}, \frac{x}{2} \frac{W}{Y}, \frac{Z}{Y}$ and $\frac{4}{x} \frac{Z}{Y}$ for use in formulas for high fre-

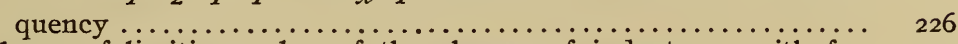

XXIII. Values of limiting value of the change of inductance with fre-

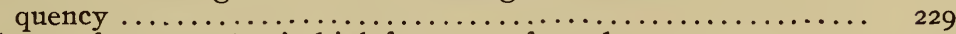

XXIV. Values of argument $x$ in high frequency formulas $\ldots \ldots \ldots \ldots \ldots \ldots . .2 \%$

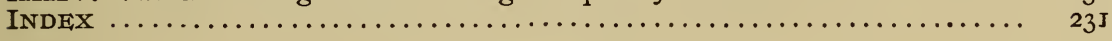

\section{INTRODUCTION}

A great many formulas have been given for calculating the mutual and self-inductance of the various cases of electrical circuits occuring in practice. Some of these formulas have subsequently been shown to be wrong, and of those which are correct and applicable to any given case there is usually a choice, because of the greater accuracy or greater convenience of one as compared with the others. For the convenience of those having such calculations to make we have brought together in this paper all the formulas with which we are acquainted which are of value in the calculation of mutual and self-inductance, particularly in nonmagnetic circuits where the frequency of the current is low enough to assure sensibly uniform distribution of current. In the last section some formulas are given for the variation of the self-inductance and resistance with the frequency. A considerable number of formulas which have been shown to be unreliable or which have been replaced by others that are less complicated or more accurate have been omitted, although in most cases we have given references to such omitted formulas. Where several formulas are applicable to the same case we have pointed out the especial advantage of each and indicated which one is best adapted to precision work.

In the second part of each section of the paper we give a number of examples to illustrate and test the formulas. We have given the work in many cases in full to serve as a guide in such calculations in order to make the formulas as useful as possible to students and others not familiar with such calculations, and also to facilitate the work of checking up the results by anyone going over the subject. We have been impressed with the importance of this in reading the work of others. 
In the appendix to the paper are a number of tables that will be found useful in numerical calculations of inductance.

In most cases we have given the name of the author of a formula in connection with the formula. This is not merely for the sake of historical interest, or to give proper credit to the authors, but also because we have found it helpful to distinguish in this way the various formulas instead of denoting each merely by a number. The formulas of sections 8 and 9, which are taken largely from a paper by one of the present authors, ${ }^{1}$ are, however, not so designated, although the authorship of those that are not new is indicated where known.

This paper includes practically all the matter contained in the I907 paper under the same title by Rosa and Cohen, but in addition to a thorough revision in which some errors are corrected and some formulas extended, a large amount of new matter has been added both in the body of the paper and in the tables. We shall be grateful to anyone detecting any errors either in formulas or tables if he communicates the same to us.

\section{MUTUAL INDUCTANCE OF TWO COAXIAL CIRCLES}

\section{MAXWELL'S FORMULAS IN ELLIPTIC INTEGRALS}

The first and most important of the formulas for the mutual inductance of coaxial circles is the formula in elliptic integrals given by Maxwell: ${ }^{2}$

$$
M=4 \pi \sqrt{A a}\left\{\left(\frac{2}{k}-k\right) F-\frac{2}{k} E\right\}
$$

in which $A$ and $a$ are the radii of the two circles, $d$ is the distance between their centers, and

$$
k=\frac{2 \sqrt{A a}}{\sqrt{(A+a)^{2}+d^{2}}}=\sin \gamma=\frac{\sqrt{r_{1}^{2}-r_{2}^{2}}}{r_{1}}
$$

$F$ and $E$ are the complete elliptic integrals of the first and second kind, respectively, to modulus $k$. Their values may be obtained from the tables of Legendre (see Tables XII and XIII in the Appendix), or the values of $M \div 4 \pi \sqrt{A a}$ may be obtained from Table $I$ in the appendix of this paper, the values of $\gamma$ being the argument.

1 This Bulletin, 4, p 30I; I907.

${ }^{2}$ Electricity and Magnetism, Vol. II, 8 7or. 
The notation of Maxwell is slightly altered in the above expressions in order to bring it into conformity with the formulas to follow.

Formula (I) is an absolute one, giving the mutual inductance of two coaxial circles of any size at any distance apart. If the two circles have equal or nearly equal radii, and are very near each other, the quantity $k$ will be very nearly equal to unity and $\gamma$ will be near to $90^{\circ}$. Under these circumstances it may be difficult to obtain a sufficiently exact value of $F$ and $E$ from the tables, as the quantities are varying rapidly and the tabular differences are very large. Under such circumstances the following formula, also given by Maxwel1 ${ }^{2}$ (derived by means of Landen's transformation), is more suitable:

$$
M=8 \pi \frac{\sqrt{A a}}{\sqrt{k_{1}}}\left\{F_{1}-E_{1}\right\}
$$

in which $F_{1}$ and $E_{1}$ are complete elliptic integrals to modulus $k_{1}$, and

$$
k_{1}=\frac{r_{1}-r_{2}}{r_{1}+r_{2}}=\sin \gamma_{1}=\frac{4 A a}{\left(r_{1}+r_{2}\right)^{2}}
$$

$r_{1}$ and $r_{2}$ are the greatest and least distances of one circle from the other (Fig. I); that is,

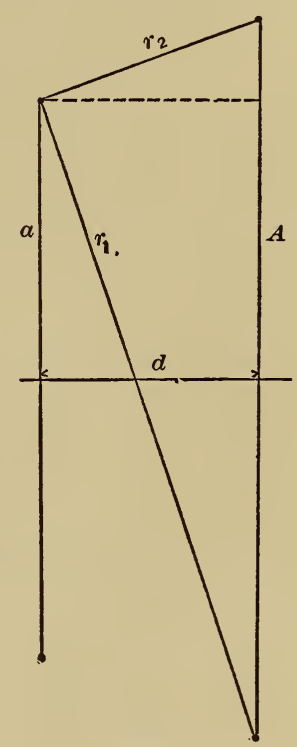

Fig. 1

$$
\begin{aligned}
& r_{1}=\sqrt{(A+a)^{2}+d^{2}} \\
& r_{2}=\sqrt{(A-a)^{2}+d^{2}}
\end{aligned}
$$

The new modulas $k_{1}$ differs from unity more than $k$, hence $\gamma_{1}$ is not so near to $90^{\circ}$ as $\gamma$ and the values of the elliptic integrals can be taken more easily from the tables than when using formula (I) and the modulus $k$.

Another way of avoiding the difficulty when $k$ is nearly unity is to calculate the integrals $F$ and $E$ directly, and thus not use the 
tables of elliptic integrals, expanding $F$ and $E$ in terms of the complementary modulus $k^{\prime}$, where $k^{\prime}=\sqrt{\mathrm{I}-k^{2}}$. $\quad k^{\prime}$ may usually be more accurately calculated by the formula $k^{\prime}=\frac{r_{2}}{r_{1}}$. The expressions for $F$ and $E$ are very convergent when $k^{\prime}$ is small.

$$
\begin{aligned}
& F=\log \frac{4}{k^{\prime}}+\frac{\mathrm{I}^{2}}{2^{2}} k^{\prime 2}\left(\log \frac{4}{k^{\prime}}-\frac{2}{\mathrm{I} \cdot 2}\right) \\
& +\frac{\mathrm{I}^{2}}{2^{2}} \frac{3^{2}}{4^{2}} k^{\prime 4}\left(\log \frac{4}{k^{\prime}}-\frac{2}{\mathrm{I} \cdot 2}-\frac{2}{3 \cdot 4}\right) \\
& +\frac{\mathrm{I}^{2}}{2^{2}} \frac{3^{2}}{4^{2}} \frac{5^{2}}{6^{2}} k^{\prime 6}\left(\log \frac{4}{k^{\prime}}-\frac{2}{\mathrm{I} \cdot 2}-\frac{2}{3 \cdot 4}-\frac{2}{5 \cdot 6}\right) \\
& +\frac{\mathrm{I}^{2}}{2^{2}} \frac{3^{2}}{4^{2}} \frac{5^{2}}{6^{2}} \frac{7^{2}}{8^{2}} k^{\prime 8}\left(\log \frac{4}{k^{\prime}}-\frac{2}{\mathrm{I} .2}-\frac{2}{3 \cdot 4}-\frac{2}{5 \cdot 6}-\frac{2}{7 \cdot 8}\right) \\
& + \\
& E=I+\frac{I}{2} k^{\prime 2}\left(\log \frac{4}{k^{\prime}}-\frac{I}{I .2}\right) \\
& +\frac{\mathrm{I}^{2}}{2^{2}} \frac{3}{4} k^{\prime 4}\left(\log \frac{4}{k^{\prime}}-\frac{2}{\mathrm{I} \cdot 2}-\frac{\mathrm{I}}{3 \cdot 4}\right) \\
& +\frac{\mathrm{I}^{2}}{2^{2}} \frac{3^{2}}{4^{2}} \frac{5}{6} k^{\prime 6}\left(\log \frac{4}{k^{\prime}}-\frac{2}{\mathrm{I} \cdot 2}-\frac{2}{3 \cdot 4}-\frac{\mathrm{I}}{5 \cdot 6}\right) \\
& +\frac{\mathrm{I}^{2}}{2^{2}} \frac{3^{2}}{4^{2}} \frac{5^{2}}{6^{2}} \frac{7}{8} k^{\prime 8}\left(\log \frac{4}{k^{\prime}}-\frac{2}{\mathrm{I} \cdot 2}-\frac{2}{3 \cdot 4}-\frac{2}{5 \cdot 6}-\frac{\mathrm{I}}{7 \cdot 8}\right) \\
& +
\end{aligned}
$$

The equations (3) are very convergent for $k^{\prime}<$ o.I, $(k \geq 0.995)$, and satisfactory accuracy will be attained down to $k=0.985$, thus covering the range of values for which interpolation in Legendre's tables becomes difficult.

For values of $k$ near 0.985 it is perhaps more accurate to calculate $M$ from elliptic integrals $F_{0}$ and $E_{0}$ with a modulus $k_{0}$ greater than $k$. The modulus $k_{0}^{\prime}$ which is complementary to $k_{0}$ is smaller than $k^{\prime}$, and the values of $F_{0}$ and $E_{0}$ calculated from the series formulas (3) putting $k_{0}^{\prime}$ in place of $k^{\prime}$ converge more rapidly than the values of $F$ and $E$ when calculated by the same series formulas. The formula for making the transformation is not quite so simple as (2). It is most conveniently written 


$$
\begin{aligned}
M & =4 \pi \sqrt{A a}\left[\frac{F_{0}}{k(\mathrm{I}+k)}-\frac{(\mathrm{I}+k)}{k} E_{0}\right] \\
k_{0}^{\prime} & =\frac{\mathrm{I}-k}{\mathrm{I}+k}=\frac{k^{\prime 2}}{(\mathrm{I}+k)^{2}} \\
k & =\frac{2 \sqrt{A a}}{\sqrt{(A+a)^{2}+d^{2}}} \quad k^{\prime}=\frac{\sqrt{(A-a)^{2}+d^{2}}}{\sqrt{\left(A+a^{2}\right)+d^{2}}}
\end{aligned}
$$

When the distance between the circles is large, formula (I) becomes unsuitable for calculation for two reasons, $(a)$ because $\gamma$ falls outside the range of Table XIII and $(b)$ because the quantity $\left(\frac{2}{k}-k\right) F-\frac{2}{k} E$ comes out as the small difference of two large quantities. The use of formula (4) overcomes the first objection, but makes the matter still worse as far as the second is concerned. We may, however, express (I) in terms of a series by means of the well k^own expressions of Wallis ${ }^{3}$

$$
\begin{aligned}
& \left.F=\frac{\pi}{2}\left[\mathrm{I}+\sum_{\mathrm{I}}^{\infty}\left\{\frac{\mathrm{I} \cdot 3 \cdot 5 \cdots \cdot(2 n-\mathrm{I})}{2 \cdot 4 \cdot 6 \cdots(2 n}\right\}^{2} k^{2 n}\right\}\right] \\
& \left.E=\frac{\pi}{2}\left[\mathrm{I}-\sum_{\mathrm{I}}^{\infty}\left\{\frac{\mathrm{I} \cdot 3 \cdot 5 \cdots(2 n-\mathrm{I})}{2 \cdot 4 \cdot 6 \cdots \cdots}\right\}^{2}\right\}^{2 n} \frac{k^{2 n}}{(2 n-\mathrm{I})}\right]
\end{aligned}
$$

Substituting these values in (I) we find

$$
M=\frac{\pi^{2} k^{3}}{4} \sqrt{A a}\left[\mathrm{I}+\frac{3}{4} k^{2}+\frac{75}{\mathrm{I} 28} k^{4}+\frac{245}{5 \mathrm{I} 2} k^{6}+\cdots\right]
$$

the general term in the brackets being

$$
\left(\frac{3 \cdot 5 \cdot 7 \cdots(2 n+I)}{4 \cdot 6 \cdot 8 \cdot \cdots(2 n+2)}\right)^{2} \frac{(2 n+2)}{(2 n-I)} k^{2 n}=\left[\frac{3 \cdot 5 \cdot 7 \cdot(2 n+I)}{4 \cdot 6 \cdot 8 \cdot \cdots 2 n}\right]^{2} \frac{k^{2 n}}{(2 n-I)(2 n+2)}
$$

For values of $k$ up to o.I $\left(\gamma=5^{\circ} \cdot 7\right)$ the series $(5)$ is very convergent, and may be used for values of $k$ up to $0.2(\gamma=\mathrm{II} \cdot 5)$ without serious labor. In the latter case and for still larger values of $k$, we may calculate $M$ in terms of the smaller modulus $k_{1}$ of formula (2). This last expression becomes on expansion 


$$
M=2 \pi^{2} k_{1}^{\frac{3}{2}} \sqrt{A a}\left[\mathrm{I}+\frac{3}{8} k_{1}{ }^{2}+\frac{\mathrm{I} 5}{64} k_{1}{ }^{4}+\frac{\mathrm{I} 75}{\mathrm{IO} 24} k_{1}{ }^{6}+\cdots .\right]
$$

the general term in the brackets being

$$
\left(\frac{n+\mathrm{I}}{2 n+\mathrm{I}}\right)\left[\frac{3 \cdot 5 \cdot 7 \cdots(2 n+\mathrm{I})}{4 \cdot 6 \cdot 8 \cdots \cdot(2 n+2)}\right]^{2} k^{2 n}
$$

The series (6) converges more rapidly than (5), and may be used with ease for values of $k_{1}$ as great as $\frac{I}{4},\left(\gamma_{1}=I 4 \cdot 5\right)$, which corresponds to $k=0.8,\left(\gamma=53^{\circ} \cdot 2\right)$.

To recapitulate-

(I) For values of $k$ between zero and 0.2 use (5).

(2) For values of $k$ a little larger and up to 0.8 use (6).

(3) For values of $k$ between about 0.7 and 0.985 the elliptic integrals in (I) may be conveniently taken by interpolation from Legendre's tables or from Table XIII.

(4) For values of $k$ greater than about 0.7 we may use (4).

(5) For values of $k$ greater than about 0.985 we may use (3).

It will be thus seen that the formulas overlap, so that it will be possible in every case to calculate the mutual inductance by at least two different formulas, the less accurate serving as a check on the more accurate.

The choice of formulas is considered more in detail on page I9.

\section{WEINSTEIN'S FORMULA}

Weinstein ${ }^{4}$ gives an expression for the mutual inductance of two coaxial circles, in terms of the complementary modulus $k^{\prime}$ used in the preceding series (3). Substituting in equation (I) the values of $F$ and $E$ given above we have Weinstein's equation, which is as follows:

$$
\begin{aligned}
M=4 \pi \sqrt{A a} & \left\{\left(\mathrm{I}+\frac{3}{4} k^{\prime 2}+\frac{33}{64} k^{\prime 4}+\frac{\mathrm{IO} 7}{25^{6}} k^{\prime 6}+\frac{59 \mathrm{I} 3}{\mathrm{I} 6384} k^{\prime 8}+\cdot\right)\left(\log \frac{4}{k^{\prime}}-\mathrm{I}\right)\right. \\
& \left.-\left(\mathrm{I}+\frac{\mathrm{I} 5}{\mathrm{I} 28} k^{\prime 4}+\frac{\mathrm{I} 85}{\mathrm{I} 536} k^{\prime 6}+\frac{7465}{65536} k^{\prime 8}+\cdots\right)\right\}
\end{aligned}
$$


This expression is rapidly convergent when $k^{\prime}$ is small, and hence will give an accurate value of $M$ when the circles are near each other. Otherwise formula (I) may be more suitable.

\section{NAGAOKA'S FORMULAS}

Nagaoka ${ }^{5}$ has given formulas for the calculation of the mutual inductance of coaxial circles, without the use of tables of elliptic integrals. These formulas make use of Jacobi's $q$-series, which is very rapidly convergent. The first is to be used when the circles are not near each other, the second when they are near each other. Either may be employed for a considerable range of distances between the extremes, although the first is more convenient. The first formula is as follows:

$$
\begin{aligned}
M & =\mathrm{I} 6 \pi^{2} \sqrt{A a} q^{\frac{3}{2}}(\mathrm{I}+\epsilon) \\
& =4 \pi \sqrt{A a}\left\{4 \pi q^{\frac{3}{2}}(\mathrm{I}+\epsilon)\right\}
\end{aligned}
$$

where $A$ and $a$ are the radii of the two circles. The correction term $\epsilon$ can be neglected when the circles are quite far apart.

$$
\begin{aligned}
& \epsilon=3 q^{4}-4 q^{6}+9 q^{8}-\mathrm{I} 2 q^{10}+\cdots \\
& q=\frac{l}{2}+2\left(\frac{l}{2}\right)^{5}+\mathrm{I}_{5}\left(\frac{l}{2}\right)^{9}+\cdots \\
& \iota=\frac{\mathrm{I}-\sqrt{k^{\prime}}}{\mathrm{I}+\sqrt{k^{\prime}}} \quad k^{\prime}=\frac{r_{2}}{r_{1}}=\frac{\sqrt{(A-a)^{2}+d^{2}}}{\sqrt{(A+a)^{2}+d^{2}}}
\end{aligned}
$$

$d$ being the distance between the centers of the circles, and $k^{\prime}$ the complementary modulus occurring in equations (3) and (7).

Nagaoka's second formula is as follows :

$$
\begin{aligned}
M & =4 \pi \sqrt{A a} \cdot \frac{\mathrm{I}}{2\left(\mathrm{I}-2 q_{1}\right)^{2}}\left\{\left[\mathrm{I}+8 q_{1}\left(\mathrm{I}-q_{1}+4 q_{1}^{2}-\cdots\right)\right] \log \frac{\mathrm{I}}{q_{1}}-4\right\}[9] \\
& =4 \pi \sqrt{A a} \cdot \frac{\mathrm{I}}{2\left(\mathrm{I}-2 q_{1}\right)^{2}}\left\{\left[\mathrm{I}+8 q_{1}-8 q_{1}^{2}+\epsilon_{1}\right] \log \frac{\mathrm{I}}{q_{1}}-4\right\} \\
q_{1} & =\frac{l_{1}}{2}+2\left(\frac{l_{1}}{2}\right)^{5}+\mathrm{I} 5\left(\frac{l_{1}}{2}\right)^{9}+\cdots \\
l_{1} & =\frac{\mathrm{I}-\sqrt{k}}{\mathrm{I}+\sqrt{k}} \quad k=\frac{2 \sqrt{A a}}{\sqrt{(A+a)^{2}+d}} \\
\epsilon_{1} & =32 q_{1}^{3}-40 q_{1}{ }^{4}+48 q_{1}^{5}-\cdots \\
-\epsilon_{1}{ }^{5} & =8 q_{1}{ }^{2}-\epsilon_{1}
\end{aligned}
$$

${ }^{5}$ Phil. Mag., 6, p. I9; I903. Recently a third expression has been found by Nagaoka (Tokyo Math. Phys. Soc., 6, p. Io; I9II). (See p. I87 below.) 
$k$ is the modulus of equation (I), but is employed here to obtain the value of the $q$-series instead of the values of the elliptic integrals employed in ( $\mathrm{I}$ ). This formula is ordinarily simpler in use than it appears, because some of the terms in the expressions above are usually negligible. For a third formula see page I87.

Nagaoka has recently published ${ }^{6}$ tables which materially reduce the labor of calculation with these formulas. These are reproduced as Tables XV and XVI of the appendix. From Table XV we obtain directly the small difference $q-\frac{l}{2}$ or $q_{1}-\frac{l_{1}}{2}$ with $q$ or $q_{1}$ as argument. The same table gives also the corresponding values of $\epsilon$ and $\log _{10}(\mathrm{I}+\epsilon)$ for use in the formula (8).

To calculate $q$ or $q_{1}$ we enter the table with $\frac{l}{2}$ or $\frac{l_{1}}{2}$ as argument. The difference corresponding in the table when added to $\frac{l}{2}$ or $\frac{l_{1}}{2}$ gives the value of $q$ or $q_{1}$ to a first approximation. This will be sufficient except for the larger values of $q$ or $q_{1}$ which are tabulated here. For these it is sometimes necessary to use this first approximation as argument to obtain a more accurate value of $q$ or $q_{1}$.

Table XVI gives the values of $\epsilon_{1}$ and $-\epsilon_{1}{ }^{\prime}$ for different values of $q_{1}$ and is useful in calculations with formula (9).

For circles at some distance from one another $q$ becomes small, and the expression for $l$ given above becomes inconvenient, because $k^{\prime}$ is so nearly equal to unity. In this case we may calculate $l$ from the somewhat more complicated expression

$$
l=\frac{k^{2}}{\left(\mathrm{I}+k^{\prime}\right)\left(\mathrm{I}+\sqrt{k^{\prime}}\right)^{2}}
$$

the values of $k$ and $k^{\prime}$ being calculated from the formulas already given. The same applies to the calculation of $l_{1}$ in formula ( $\left.9 a\right)$, when the circles are very near together, and consequently $q_{1}$ is very small. For this case we use the expression

$$
l_{1}=\frac{k^{\prime 2}}{(\mathrm{I}+k)(\mathrm{I}+\sqrt{k})^{2}}
$$

${ }^{6}$ Jour. of Coll. of Sci. Tokyo, vol. 27, art. 6; 1909. 


\section{MAXWELL'S SERIES FORMULA}

Maxwe $11^{7}$ obtained an expression for the mutual inductance between two coaxial circles in the form of a converging series which is often more convenient to use than the elliptical integral formula, and when the circles are nearly of the same radii and relatively near each other the value given is generally sufficiently exact. In the following formula $a$ is the smaller of the two radii, $c$ is their difference, $A-a, d$ is the distance apart of the circles as before, and $r=\sqrt{c^{2}+d^{2}}$. The mutual-inductance is then

$$
\begin{aligned}
M= & 4 \pi \alpha\left\{\log \frac{8 a}{r}\left(\mathrm{I}+\frac{c}{2 a}+\frac{c^{2}+3 d^{2}}{\mathrm{I} 6 a^{2}}-\frac{c^{3}+3 c d^{2}}{32 a^{3}}+. . .\right)\right. \\
& \left.-\left(2+\frac{c}{2 a}-\frac{3 c^{2}-d^{2}}{\mathrm{I} 6 a^{2}}+\frac{c^{3}-6 c d^{2}}{48 a^{3}}-. . .\right)\right\}
\end{aligned}
$$

When $c$ and $d$ are small compared with $a$, we have for an approximate value of the mutual inductance the following simple expression: ${ }^{8}$

$$
M=4 \pi a\left\{\log \frac{8 a}{r}-2\right\}[\mathrm{II}]
$$

When the two radii are equal, as is often the case in practice, the equation (IO) is somewhat simplified, as follows:

$$
M=4 \pi a\left\{\log \frac{8 a}{d}\left(\mathrm{I}+\frac{3 d^{2}}{\mathrm{I} 6 a^{2}}\right)-\left(2+\frac{d^{2}}{\mathrm{I} 6 a^{2}}\right)\right\}
$$

The above formulas (IO) and (I2) are sufficiently exact for very many cases, the terms omitted in the series being unimportant when $\frac{c}{a}$ and $\frac{d}{a}$ are small. For example, if

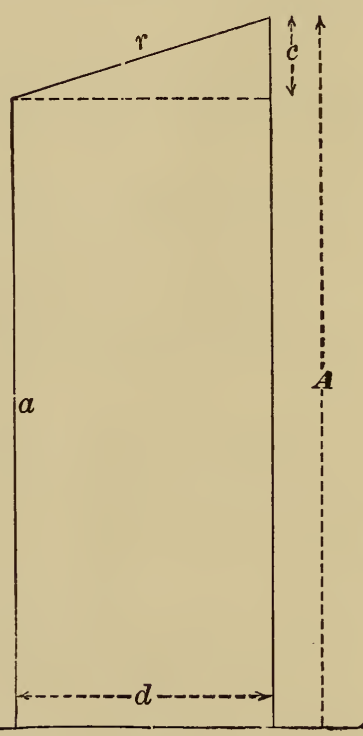

Fig. 2 $\frac{d}{d}$ is o.I, the largest term neglected in (I 2) is less than two parts in a million. If, however, $d=a$, this term will be more than one per cent, and the formula will be quite inexact.

${ }^{7}$ Electricity and Magnetism, Vol. II, \& 705.

8 This is equivalent to the approximate formula given by Wiedemann, $M=4 \pi a\left\{\log \frac{2 l}{c}-2.45\right\}$, where $l$ is the circumference of the smaller circle and $c$ is the same as $r$ above. 
Coffin ${ }^{9}$ has extended Maxwell's formula (I2) for two equal circles by computing three additional terms for each part of the expression. This enables the mutual inductance to be computed with considerable exactness up to $d=\alpha$. Formula ( $\mathrm{I}$ ) is exact, as stated above, for all distances, and either it or (8) should be used in preference to (I3) when $d$ is large. Coffin's formula is as follows:

$$
\begin{aligned}
M= & 4 \pi a\left\{\log \frac{8 a}{d}\left(\mathrm{I}+\frac{3 d^{2}}{\mathrm{I} 6 a^{2}}-\frac{\mathrm{I} 5 d^{4}}{8 \times \mathrm{I} 28 a^{4}}+\frac{35 d^{6}}{1228^{2} a^{6}}-\frac{\mathrm{I} 575 d^{8}}{2 \times 128^{3} a^{8}}+\cdots\right)\right. \\
& \left.-\left(2+\frac{d^{2}}{\mathrm{I} 6 a^{2}}-\frac{3 \mathrm{I} d^{4}}{\mathrm{I} 6 \times \mathrm{I} 28 a^{4}}+\frac{247 d^{6}}{6 \times{\mathrm{I} 28^{2} a^{6}}^{6}}-\frac{7795 d^{8}}{8 \times \mathrm{I} 28^{3} a^{8}}+\cdots\right)\right\}[\mathrm{I} 3]
\end{aligned}
$$

We have extended Maxwell's formula (Io) for unequal circles as follows: ${ }^{10}$

$$
\begin{aligned}
M= & 4 \pi a\left\{\operatorname { l o g } \frac { 8 a } { r } \left(\mathrm{I}+\frac{c}{2 a}+\frac{c^{2}+3 d^{2}}{\mathrm{I} 6 a^{2}}-\frac{c^{3}+3 c d^{2}}{32 a^{3}}+\frac{\mathrm{I} 7 c^{4}+42 c^{2} d^{2}-\mathrm{I} 5 d^{4}}{\mathrm{IO} 24 a^{4}}\right.\right. \\
& \left.-\frac{\mathrm{I} 9 c^{5}+30 c^{3} d^{2}-45 c d^{4}}{2048 a^{5}}+\cdots\right)-\left(2+\frac{c}{2 a}-\frac{3 c^{2}-d^{2}}{\mathrm{I} 6 a^{2}}+\frac{c^{3}-6 c d^{2}}{48 a^{3}}\right. \\
& \left.\left.+\frac{\mathrm{I} 9 c^{4}+534 c^{2} d^{2}-93 d^{4}}{6 \mathrm{I} 44 a^{4}}-\frac{379 c^{5}+3030 c^{3} d^{2}-\mathrm{I} 845 c d^{4}}{6 \mathrm{I} 440 a^{5}}\right)\right\}
\end{aligned}
$$

Nagaoka ${ }^{11}$ has confirmed this extension by expanding formula (9). He carried out the expansion, however, no further than terms in $\frac{c^{4}}{a^{4}}$ and $\frac{d^{4}}{a^{4}}$.

When $c=0$, this gives the first part of series (I 3 ). When $d=0$, the case of two circles in the same plane, with radii $a$ and $a+c$, we have

$$
\begin{array}{r}
M=4 \pi a\left\{\log \frac{8 a}{c}\left(\mathrm{I}+\frac{c}{2 a}+\frac{c^{2}}{\mathrm{I} 6 a^{2}}-\frac{c^{3}}{32 a^{3}}+\frac{\mathrm{I} 7 c^{4}}{\mathrm{IO} 24 a^{4}}-\frac{\mathrm{I} 9 c^{5}}{2048 a^{5}}+\cdots\right)\right. \\
\left.-\left(2+\frac{c}{2 a}-\frac{3 c^{2}}{\mathrm{I} 6 a^{2}}+\frac{c^{3}}{48 a^{3}}+\frac{\mathrm{I} 9 c^{4}}{6 \mathrm{I} 44 a^{4}}-\frac{379 c^{5}}{6 \mathrm{I} 440 a^{5}}+\cdots\right)\right\} \quad[\mathrm{I} 5]
\end{array}
$$

${ }^{9}$ J. G. Coffin, this Bulletin, 2, p. I I3; 1906.

${ }_{10}$ This Bulletin, 2, p. 364 ; 1906.

${ }^{11}$ Loc. cit., p. II. 
These formulas (14) and (I5) give the mutual inductance with great precision when the circles are not too far apart. The degree of convergence, of course, indicates approximately in any case the accuracy of the result.

\section{HAVELOCK'S FORMULA}

In 1908 Havelock $^{12}$ published a paper in which the calculation of mutual and self-inductance is made to depend on the evaluation of certain definite integrals of Bessel functions of the form $\int_{0}^{\infty} e^{-p \mu} J_{1}(\mu) J_{1}(\lambda \mu) \mu^{-n} d \mu$. These he expands in the form of series, which fall into two classes, those suitable for sinall values of $p$, and those suitable for large values of $p$. In the case of the latter, he gives the expressions for the general terms of the series, so that these may be extended as far as desired. In the case of the former only a few terms are given, and the derivation of further terms is very tedious.

He considers first the mutual inductance of two coaxial circles, and points out that the solution may be made to depend on either of two of his integrals. He does not, however, write out the formulas. It is a simple matter to carry out the necessary substitutions, and we find for circles near one another

$$
\begin{gathered}
M=4 \pi \sqrt{A a}\left\{\left[\mathrm{I}+\frac{3}{\mathrm{I} 6}\left(\frac{r^{2}}{A a}\right)-\frac{\mathrm{I} 5}{\mathrm{IO} 24}\left(\frac{r^{2}}{A a}\right)^{2}+\cdots \cdot\right] \log \frac{8 \sqrt{A a}}{r}\right. \\
\left.-\left(2+\frac{\mathrm{I}}{\mathrm{I} 6}\left(\frac{r^{2}}{A a}\right)-\frac{3 \mathrm{I}}{2048}\left(\frac{r^{2}}{A a}\right)^{2}+\cdots \cdot\right)\right\}
\end{gathered}
$$

This expression bears some resemblance to Maxwell's series formula (ro); it is, however, simpler for use in calculation. To obtain the coefficients of further terms by Havelock's process would require a good deal of labor. We notice, however, that, putting $A=a$, the formula becomes the same as Coffin's formula (I 3 ) for equal circles. We may evidently, therefore, use the coefficients of the higher order terms in Coffin's formula to obtain an extension of the above, and find the expression 
$\begin{array}{cc}M=4 \pi \sqrt{A \alpha}\left\{\left[\mathrm{I}+\frac{3}{\mathrm{I} 6} \alpha-\frac{\mathrm{I} 5}{\mathrm{IO} 24} \alpha^{2}+\frac{35}{\mathrm{I} 28^{2}} \alpha^{3}-\frac{\mathrm{I} 575}{2 \times \mathrm{I}_{28}^{3}} \alpha^{4}+\cdots\right] \log \frac{8 \sqrt{A \alpha}}{r}\right. \\ \left.-\left(2+\frac{\mathrm{I}}{\mathrm{I} 6} \alpha-\frac{3 \mathrm{I}}{2048} \alpha^{2}+\frac{247}{6 \times \overline{\mathrm{I} 28^{8}} \alpha^{3}}-\frac{7795}{8 \times \overline{\mathrm{I} 28^{3}}} \alpha^{4}+\cdots\right)\right\} & {[\mathrm{I} 6]}\end{array}$

where

$$
r^{2}=c^{2}+d^{2} \quad \alpha=\frac{a}{A} \frac{r^{2}}{a^{2}}
$$

The expression thus extended ${ }^{13}$ gives very accurate results for values of $d$ almost as great as the radius $a$. For a given degree of convergence it requires only half as many terms to be calculated as does formula (I4), and is much easier to calculate.

The second formula derived from Havelock's paper is not so generally useful, being rapidly convergent only for values of $d$ greater than about $5 A$. It is

$$
\begin{aligned}
M=\frac{2 \pi^{2} a^{2} A^{2}[}{d^{3}}[\mathrm{I} & -\frac{3}{2}\left(\mathrm{I}+\frac{a^{2}}{A^{2}}\right) \frac{A^{2}}{d^{2}}+\frac{\mathrm{I} 5}{8}\left(\mathrm{I}+3 \frac{a^{2}}{A^{2}}+\frac{a^{4}}{A^{4}}\right) \frac{A^{4}}{d^{4}} \\
& -\frac{35}{\mathrm{I} 6}\left(\mathrm{I}+6 \frac{a^{2}}{A^{2}}+6 \frac{a^{4}}{A^{4}}+\frac{a^{6}}{A^{6}}\right) \frac{A^{6}}{d^{6}} \\
& +\frac{3 \mathrm{I} 5}{\mathrm{I} 28}\left(\mathrm{I}+\mathrm{I0} \frac{a^{2}}{A^{2}}+20 \frac{a^{4}}{A^{4}}+\mathrm{IO} \frac{a^{6}}{A^{6}}+\frac{a^{8}}{A^{8}}\right) \frac{A^{8}}{d^{8}} \\
& \left.-\frac{693}{256}\left(\mathrm{I}+\mathrm{I} 5 \frac{a^{2}}{A^{2}}+50 \frac{a^{4}}{A^{4}}+50 \frac{a^{6}}{A^{6}}+\mathrm{I} 5 \frac{a^{8}}{A^{8}}+\frac{a^{10}}{A^{10}}\right) \frac{A^{10}}{d^{10}}+\cdots\right]
\end{aligned}
$$

For the case of $d=\operatorname{IO} A$ and $a$ as great as $A$, only three terms have to be calculated to obtain $M$ to about one part in a million, and for a smaller value of $\frac{a}{A}$ the convergence would be more rapid still.

\section{MATHY'S FORMULA}

In an interesting paper in the Journal de Physique for I90I, ${ }^{14} \mathrm{E}$. Mathy obtained a formula for the mutual inductance of two circles,

${ }^{13} \mathrm{Mr}$. T. J. Bromwich, of Cambridge, England, has recently communicated to us the same formula, without giving the proof, including however terms no higher than those in $\alpha^{3}$.

${ }^{14}$ Jour. de Phys., 10, p. 33; rgor. 
in which the elliptic integral of the third kind, on which the mutual inductance depends, is expanded in a manner still different from that adopted in any of the preceding cases. It is expressed in terms of hypergeometric series involving the absolute invariant $J$ of the Weierstrassian $\mathbf{p}$ function. The final expression as found by Mathy is incorrect as regards the coefficients of the hypergeometric series. The corrected expression, ${ }^{15}$ using the notation of this paper, is as follows :

$$
\begin{gathered}
\frac{M}{4 \pi}=\left[\frac { x ^ { 2 } } { ( x ^ { 4 } + \mathrm { I } 2 A ^ { 2 } a ^ { 2 } ) ^ { \frac { 1 } { 4 } } } \left(\frac{P}{\sqrt[4]{27}} F\left(\frac{\mathrm{I}}{\mathrm{I} 2}, \frac{5}{\mathrm{I} 2}, \frac{\mathrm{I}}{2}, \frac{J-\mathrm{I}}{J}\right)-\frac{Q}{6 \sqrt[4]{3}} \sqrt{\frac{J-\mathrm{I}}{J}}\right.\right. \\
\left.F\left(\frac{7}{\mathrm{I} 2}, \frac{\mathrm{II}}{\mathrm{I} 2}, \frac{3}{2}, \frac{J-\mathrm{I}}{J}\right)\right\}-\left(x^{4}+\mathrm{I} 2 A^{2} a^{2}\right)^{\frac{1}{4}}\left\{\frac{Q}{\sqrt[4]{3}} F\left(-\frac{\mathrm{I}}{\mathrm{I} 2}, \frac{7}{\mathrm{I} 2}, \frac{\mathrm{I}}{2}, \frac{J-\mathrm{I}}{J}\right)\right. \\
\left.\left.+\frac{P}{6 \sqrt[4]{27}} \sqrt{\frac{J-\mathrm{I}}{J}} F\left(\frac{5}{\mathrm{I} 2}, \frac{\mathrm{I}}{\mathrm{I} 2}, \frac{3}{2}, \frac{J-\mathrm{I}}{J}\right)\right\}\right]
\end{gathered}
$$

where

$$
\begin{aligned}
x^{2} & =a^{2}+A^{2}+d^{2} \\
P & =\mathrm{I} .3 \mathrm{II02} 8777 \ldots . \\
Q & =0.599070 \mathrm{II} 7 \ldots . \\
\sqrt{\frac{J-\mathrm{I}}{J}} & =\frac{\mathrm{I}-36\left(\frac{a A}{x^{2}}\right)^{2}}{\left[\mathrm{I}+\mathrm{I} 2\left(\frac{a A}{x^{2}}\right)^{2}\right]^{\frac{3}{2}}}
\end{aligned}
$$

and

$$
\begin{aligned}
F(\alpha, \beta, \gamma, z)=\mathrm{I} & +\frac{\alpha \beta}{\mathrm{I} \cdot \gamma} z+\frac{\alpha(\alpha+\mathrm{I}) \cdot \beta(\beta+\mathrm{I})}{\mathrm{I} \cdot 2 \cdot \gamma(\gamma+\mathrm{I})} z^{2} \\
& +\frac{\alpha(\alpha+\mathrm{I})(\alpha+2) \cdot \beta(\beta+\mathrm{I})(\beta+2)}{\mathrm{I} \cdot 2 \cdot 3 \cdot \gamma(\gamma+\mathrm{I})(\gamma+2)} z^{3}+\cdots
\end{aligned}
$$

This formula is by no means so formidable to use as might be expected, since the constants which enter and the coefficients in the hypergeometric series may be calculated once for all. Using seven place logarithms we find 


$$
\begin{array}{ll}
\log _{10} \frac{P}{\sqrt[4]{27}}=9.75977 \mathrm{r} 2 & \log _{10} \frac{P}{6 \sqrt[4]{27}}=8.9816199 \\
\log _{10} \frac{Q}{\sqrt[4]{3}}=9.6581974 & \log _{10} \frac{Q}{6 \sqrt[4]{3}}=8.880046 \mathrm{I}
\end{array}
$$

The coefficients $a_{1}, a_{2}, a_{3}$ in each of the four series are given in Table XVII. For practical purposes the formula should be used only for values of $\sqrt{\frac{J-I}{J}}$ smaller than about 0.2.

For the special case $\sqrt{\frac{J-1}{J}}=0$, it is of interest to note that the mutual inductance is given by the simple expression

$$
\frac{M}{4 \pi}=\left[\frac{x^{2}}{\left(x^{4}+\mathrm{I} 2 A^{2} a^{2}\right)^{\frac{1}{4}}} \cdot \frac{P}{\sqrt[4]{27}}-\left(x^{4}+\mathrm{I} 2 A^{2} a^{2}\right)^{\frac{1}{4}} \cdot \frac{Q}{\sqrt[4]{3}}\right]
$$

which, remembering that $x^{4}=36 A^{2} a^{2}$ in this case, becomes

$$
\begin{aligned}
M & =4 \pi \sqrt{A a}(P-2 Q)=4 \pi(\text { O.I } 2888542) \sqrt{A a} \\
& =1.418599262 \sqrt{A a}
\end{aligned}
$$

If we introduce the distances $r_{1}$ and $r_{2}$ (Fig. I) into the formula for $\sqrt{\frac{J-I}{J}}$, we see that the necessary and sufficient condition that this remarkably simple formula ${ }^{15 a}$ may be used is that $r_{1}{ }^{2}=2 r_{2}{ }^{2}$, or $k^{\prime}=k=\frac{\mathrm{I}}{\sqrt{2}}$. That is, the greatest distance between the two circumference must be $\sqrt{2}$ times the shortest distance between them. The most important cases satisfying this condition are

$$
\begin{array}{ccl}
\frac{a}{A} & d & \\
\mathrm{I} & 2 A & \text { Equal circles. } \\
3-2 \sqrt{2} & 0 & \text { Circles in the same plane. } \\
\frac{\mathrm{I}}{2} & \frac{\mathrm{I}}{2} \sqrt{7} A &
\end{array}
$$

${ }^{15 a}$ Nagaoka has recently shown (Tokyo Math. Phys. Soc., 6, p. IO; I9II) that formula (I9) may be derived from Maxwell's formula (I). 
The convergence of the formula (I8) will of course be satisfactory for moderate deviations on the either side of the ideal ratio of $\frac{d}{A}$, but the formula must be regarded as of more limited application than most of those above. It gives, however, a very rapid and accurate means of checking other formulas, since in the ideal case the mutual inductance can be calculated by (I9) to any number of decimal places desired, according to the number of figures retained in Stirling's constants $P$ and $Q$.

\section{CHOICE OF FORMULAS}

With so many to choose among, it is possible to select a favorable formula for any individual case. For this purpose $r_{1}$ and $r_{2}$, the longest and shortest distances between the circles, need to be considered, since on their relative values the convergence or convenience of the various formulas for calculation depends. The following table gives roughly the range of values of the ratio $\frac{r_{2}}{r_{1}}$ within which the different formulas are capable of giving the best results. Since, however, the determination of such limits is somewhat arbitrary, the values given here should not be regarded as more than a guide. In the case of those formulas which occur in the form of a series the limiting value of the ratio $\frac{r_{2}}{r_{1}}$ has been calculated which makes the last term included not greater than one ten-thousandth of the whole. The values of $\frac{r_{2}}{r_{1}}$ for Nagaoka's formulas have been calculated for the limits of his correction tables.

\section{SUMMARY OF FORMULAS FOR CIRCLES}

Formula

Weinstein's

Maxwell's

"

"6

"6

6
Range of values

$$
\text { of } \frac{r_{2}}{r_{1}}
$$

$\begin{array}{ll}0 & \text { to } 0.25 \\ 0 & \text { to } 0.02 \\ 0 & \text { to } 0.14 \\ 0 & \text { to } 0.22 \\ 0 & \text { to } 0.2 \\ 0.02 & \text { to } 0.20\end{array}$

o to 0.25

0 to 0.02

o to 0.14

$\mathrm{O}$ to 0.22

0.02 to 0.20
Most favorable values of $\frac{d}{A}$ for equal circles

$\begin{array}{ll}0 & \text { to } 0.5 \\ 0 & \text { to } 0.04 \\ 0 & \text { to } 0.3 \\ 0 & \text { to } 0.45 \\ 0 & \text { to } 0.4 \\ 0.04 \text { to } 0.4\end{array}$


Formula

Havelock's

Coffin's

Nagaoka's

Maxwell's

"،

Mathy

"6

Nagaoka's

Maxwell's

Havelock's

Maxwell's
Range of values

$$
\text { of } \frac{r_{2}}{r_{1}}
$$

(5) o to 0.4

0.04 to 0.4

o to 0.75

0.2 to 0.7

0.65 to 0.75

$$
\frac{I}{2} \sqrt{2}
$$

0.3 to $\mathrm{I}$

0.6 to $\mathrm{I}$

0.9 to $I$

0.98 to I
Most favorable values of $\frac{d}{A}$ for equal circles

o to 0.9

o to 0.9

0.08 to 0.9

o to 2.25

0.4 to 2

I. 7.5 to 2.25

2

greater than 0.6

" " " I.5

" 14

"6 6 IO

EXAMPLES TO ILLUSTRATE AND TEST THE FORMULAS EXAMPLE 1. MAXWELL'S FORMULA (1). FOR ANY COAXIAL CIRCLES

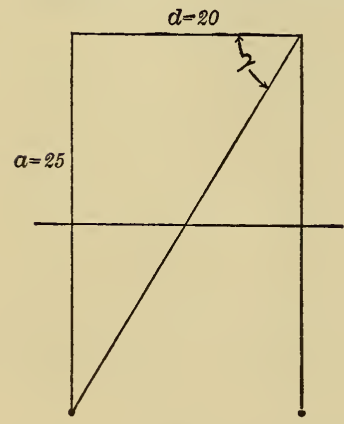

Fig. 3
Let $a=A=25 \mathrm{~cm}$, Fig. 3 , $d=20 \mathrm{~cm}$.

$$
\begin{aligned}
& k=\frac{50}{\sqrt{2500+400}}=0.9284766=\sin \gamma \\
& \gamma=68^{\circ} \mathrm{II}^{\prime} 54^{\prime \prime} 88=68 .{ }^{\circ} \mathrm{I} 98578 .
\end{aligned}
$$

From Legendre's tables, we obtain $\begin{aligned} & \log F=0.3852191 \\ & \log E=0.0547850\end{aligned} \quad\left(\frac{2}{k}-k\right) F-\frac{2}{k} E=0.5318500$ $4 a=100$ $\therefore \quad M=167.08562 \mathrm{~cm}$.

To facilitate calculations in such problems as this, we have prepared Table II, which gives $F$ and $\log F, E$ and $\log E$, as functions of $\tan \gamma$. In the above case $\tan \gamma=\frac{50}{20}=2.5$, and from Table II we can take the values of $\log F$ and $\log E$ directly, avoiding the calculation of $\gamma$ and the interpolation for $\log F$ and $\log E$ in Legendre's tables (or Table XIII). This is only applicable for circles of equal radii, and is especially advantageous when $\tan \gamma$ is one of the values given in the table, when interpolation is unnecessary. 
The above problem may also be calculated by means of Table I, taken from Maxwell, as follows :

$$
\begin{aligned}
\log _{10} \frac{M}{4 \pi a} \text { for } 68: \mathrm{I} & =\overline{\mathrm{I}} \cdot 7230634 \\
\text { for } 68: 2 & =\underline{\overline{\mathrm{I}} .725828 \mathrm{I}} \\
\text { for } 68: \mathrm{I} 98578 & =\overline{\mathrm{I}} \cdot 7257888=\log \frac{M}{4 \pi a}
\end{aligned}
$$

$\therefore M=167.08546 \mathrm{~cm}$, agreeing almost exactly with the above value.

The calculation of mutual inductance by the above methods is simplest for circles not near each other, as then the values of $\log F$, $\log E$, and $\log \frac{M}{4 \pi \sqrt{A a}}$ are very exact when taken by simple interpolation. When $\gamma$ is nearly $90^{\circ}$, however, second and third differences have to be used in interpolation.

EXAMPLE 2. MAXWELL'S SECOND EXPRESSION (2). FOR CIRCLES NEAR EACH OTHER

$$
\text { Let } a=A=25 \mathrm{~cm}, d=\mathrm{I} \mathrm{cm}
$$

In this case $k=\sin \gamma=\frac{50}{\sqrt{25 \mathrm{OI}}}=0.9998002 \quad \gamma=88^{\circ} 5^{\mathrm{I}^{\prime}} \mathrm{I}^{\prime \prime}$

This value of $\gamma$ is so nearly $90^{\circ}$ that it is difficult to obtain accurate values of $F$ and $E$ frum tables of elliptic integrals, or of $\frac{M}{4 \pi a}$ from Maxwell's table.

We may therefore use formula (2) instead of (I).

$$
\begin{aligned}
r_{1} & =\sqrt{2501}=50.01 \text { nearly, } r_{2}=1.0 \\
\therefore k_{1} & =\sin \gamma_{1}=\frac{4 A a}{\left(r_{1}+r_{2}\right)^{2}}=0.9607920 \\
\gamma_{1} & =73^{\circ} 54^{\prime} 9 .^{\prime \prime} 67=73^{\circ} \cdot 902687
\end{aligned}
$$

From Legendre's tables for $\gamma_{1}=73^{\circ} \cdot 902687, F_{1}=2.7024545$ or Table XIII, $\} \quad E_{1}=\underline{\text { I.08 }} 52$ I 70

$$
F_{1}-E_{1}=\overline{\mathrm{I} .6 \mathrm{I} 72375}
$$

$$
\frac{8 \pi \sqrt{A a}}{\sqrt{k_{1}}}=\frac{200 \pi}{\sqrt{.9607920}} \therefore \frac{8 \pi \sqrt{A a}}{\sqrt{k_{1}}}\left(F_{1}-E_{1}\right)=M=1036.6664 \mathrm{~cm} .
$$


EXAMPLE 3. FORMULA (3). SERIES FOR F AND E, CIRCLES NEAR EACH OTHER

Suppose that, in the last example, we calculate $F$ and $E$ by means of formula (3), instead of taking them from Table XIII.

$$
\begin{gathered}
A=a=25, d=\mathrm{I} . \\
k^{\prime 2}=\mathrm{I}-k^{2}=\mathrm{I}-\frac{2500}{25 \mathrm{OI}}=\frac{\mathrm{I}}{25 \mathrm{OI}} \\
\therefore F=5.298947 \mathrm{I} E=\mathrm{I} .0009594
\end{gathered}
$$

If these values of $F$ and $E$ be substituted in formula (I), $k$ being 0.9998002 , we obtain $M=1036.6652$, which is very closely the same value as by formula (2).

EXAMPLE 4. FORMULA (3). SECOND CASE, CIRCLES NOT NEAR

$$
\begin{gathered}
A=25, a=20, d=\text { IO cm. (See Fig. I.) } \\
k^{2}=\frac{4 \times 20 \times 25}{(45)^{2}+(\mathrm{IO})^{2}}=\frac{\mathrm{I} 6}{\mathrm{I} 7} \therefore k^{\prime 2}=\frac{\mathrm{I}}{\mathrm{I} 7}
\end{gathered}
$$

$\log \frac{4}{k^{\prime}}=\frac{\mathrm{I}}{2} \log (\mathrm{I} 6 \times \mathrm{I} 7)=\frac{\mathrm{I}}{2} \log _{e} 272$

$=2.8029010$

$$
\begin{aligned}
\frac{k^{\prime 2}}{4}\left(\log \frac{4}{k^{\prime}}-\mathrm{I}\right) & =.0265 \mathrm{I} 32 \\
\frac{9 k^{\prime 4}}{64}\left(\log \frac{4}{k^{\prime}}-\frac{7}{6}\right) & =.0007962 \\
\frac{25 k^{\prime 6}}{25^{6}}\left(\log \frac{4}{k^{\prime}}-\frac{\mathrm{III}}{90}\right) & =.00003 \mathrm{I} 2 \\
\frac{\mathrm{I} 225^{\prime 8}}{\mathrm{I} 6384}\left(\log \frac{4}{k^{\prime}}-\mathrm{I} .27\right) & =.00000 \mathrm{I} 4 \\
\mathrm{I}+\frac{k^{\prime 2}}{2}\left(\log \frac{4}{k^{\prime}}-\frac{\mathrm{I}}{2}\right) & =2.8302430 \\
\frac{3 k^{\prime 4}}{\mathrm{I} 6}\left(\log \frac{4}{k^{\prime}}-\frac{\mathrm{I} 3}{\mathrm{I} 2}\right) & =.00677324 \\
\frac{\mathrm{I} 5 k^{\prime 6}}{\mathrm{I} 28}\left(\log \frac{4}{k^{\prime}}-\mathrm{I} .20\right) & =.000038 \mathrm{II} 56 \\
\frac{\mathrm{I} 75^{\prime 8}}{2048}\left(\log \frac{4}{k^{\prime}}-\mathrm{I} .25\right) & =.00000 \mathrm{I} 7 \\
\therefore E & =\mathrm{I} .0688878
\end{aligned}
$$


To find the value of $M$ we now use equation (I).

$$
\left\{\left(\frac{2}{k}-k\right) F-\frac{2}{k} E\right\}=0.885388
$$

Multiplying by $4 \pi \sqrt{A a}=4 \pi \sqrt{500}$ gives

$$
M=248.7875 \mathrm{~cm} \text {. }
$$

EXAMPLE 5. FORMULA (4). CIRCLES NEAR TOGETHER

$$
\begin{gathered}
A=a=25 \quad d=4 \\
k=\frac{2 \sqrt{A a}}{\sqrt{(A+a)^{2}+d^{2}}}=\frac{50}{\sqrt{25 \mathrm{I} 6}}=0.9968 \mathrm{I} 54 \\
k^{\prime 2}=\frac{(A-a)^{2}+d^{2}}{(A+a)^{2}+d^{2}}=\frac{\mathrm{I} 6}{25 \mathrm{I} 6}=0.00635930 \mathrm{I} 5 \\
k_{0}^{\prime}=\frac{k^{\prime 2}}{(\mathrm{I}+k)^{2}} \quad=0.0015949004 \\
\log _{e} \frac{4}{k_{0}^{\prime}}=\log _{e} 2507.9937=7.8272373 \\
\frac{k_{0}^{\prime 2}}{4}\left(\log _{e} \frac{4}{k_{0}^{\prime}}-\mathrm{I}\right) \quad=\frac{0.0000043}{7.82724 \mathrm{I} 6}=F_{0} \\
\mathrm{I}+\frac{k_{0}^{\prime 2}}{2}\left(\log _{e} \frac{4}{k_{0}^{\prime}}-\frac{\mathrm{I}}{2}\right)=\mathrm{I} .0000093=E_{0} \\
\frac{F_{0}}{k(\mathrm{I}+k)}=3.9323856 \\
\frac{E_{0}}{k}(\mathrm{I}+k)=\frac{2.0032 \mathrm{I} 34}{\mathrm{I} .929 \mathrm{I} 722}
\end{gathered}
$$

Multiplying by $4 \pi \sqrt{A a}$ gives

$$
M=606.0674 \mathrm{~cm} \text {. }
$$

If we calculate $M$ by formula (3) we find that, to obtain the same precision, terms in $k^{\prime 4}$ in the series for $F$ and $E$ have to be included, and we find

$$
M=606.0678^{\circ} \mathrm{cm}
$$


EXAMPLE 6. FORMULA (5). CIRCLES FAR APART

$$
\begin{array}{rl}
A=a=\mathrm{IO} & d=\mathrm{IOO} \\
k=\frac{2 \mathrm{O}}{\sqrt{\mathrm{IO} 4 \mathrm{OO}}}=\frac{\mathrm{I}}{\sqrt{26}} & =0 . \mathrm{I} 96 \mathrm{II} 6 \mathrm{I} 5 \\
\mathrm{I}+\frac{3}{4} k^{2} & =\mathrm{I} .028846 \mathrm{I} 6 \\
\frac{75}{\mathrm{I} 28} k^{4} & =0.00086684 \\
\frac{245}{5^{\mathrm{I} 2}} k^{6} & =0.00002723 \\
\frac{66 \mathrm{I} 5}{\mathrm{I} 28^{2}} k^{8} & =0.00000088 \\
\mathrm{Sum} & =\mathrm{I} .02974 \mathrm{III} \\
\log \mathrm{sum} & =0.012728 \mathrm{I} \\
\log k^{3} & =\overline{3} .8775400 \\
\log \frac{\pi^{2} \sqrt{A a}}{4} & =\underline{\mathrm{I} .3922398} \\
\log M & =\overline{\mathrm{I}} .2825079 \\
M & =0.19 \mathrm{I} 64962 \mathrm{~cm} .
\end{array}
$$

If formula ( $\mathrm{\tau}$ ) be used, and the values of $F$ and $E$ be taken by interpolation from Table XII, the value $M=0.19 I 643$ is found, which is in error by more than 5 parts in roooo. Using the formula (6) terms in $k_{1}^{2}$ only need be calculated, and we find $M=0.1916495^{8}$, which differs by only one part in five million from the value given by (5).

EXAMPLE 7. FORMULA (6). CIRCLES NOT NEAR TOGETHER

$$
\begin{aligned}
& A=25 \quad a=20 \quad d=40 \\
& r_{1}=\sqrt{3625} \quad r_{2}=\sqrt{\mathrm{I} 625} \\
& k_{1}=\frac{4 A a}{\left(r_{1}+r_{2}\right)^{2}}=0.19793905
\end{aligned}
$$




$$
\begin{array}{rrr}
\mathrm{I}+\frac{3}{8} k_{1}{ }^{2}=\mathrm{I} .0 \mathrm{I} 469245 & \log \text { sum }=0.0064929 \\
\frac{\mathrm{I} 5}{64} k_{1}{ }^{4}=0.00035978 & \log k_{1}{ }^{\frac{3}{2}}=\overline{2} .9447972 \\
\frac{\mathrm{I} 75}{\mathrm{IO} 24} k_{1}{ }^{6}=0.0000 \mathrm{IO} 28 & \log \frac{\pi}{2} \sqrt{A a}=\underline{\mathrm{I} .5456049} \\
\frac{\mathrm{I} 225}{\mathrm{I} 28^{2}} k_{1}{ }^{8}=0.00000032 & \log \frac{M}{4 \pi}=0.4968950 \\
\text { Sum }=\mathrm{I} .0150628 &
\end{array}
$$$$
\therefore \frac{M}{4 \pi}=3.1397496 \mathrm{~cm}
$$

By formula (8) $\quad \frac{M}{4 \pi}=3.1397486$

If formula (I) is used and the elliptic integrals be taken from Table XII by interpolation the value $\frac{M}{4 \pi}=3 \cdot 1397656$ is found, which is only five in a million in error.

\section{EXAMPLE 8. WEINSTEIN'S FORMULA (7). FOR ANY COAXIAL CIRCLES} NOT TOO FAR APART

Take the same circles as in example 4 .

$$
\begin{aligned}
& A=25, a=20, c=5, d=\mathrm{IO} \\
& k^{\prime 2}=\frac{\mathrm{I}}{\mathrm{I} 7}, \log \frac{4}{k^{\prime}}-\mathrm{I}=\mathrm{I} .80290 \mathrm{I} \\
& \mathrm{I}+\frac{3}{4} k^{\prime 2}=\mathrm{I} .044 \mathrm{II} 76 \\
& \mathrm{I}+\frac{\mathrm{I} 5}{\mathrm{I} 28} k^{\prime 4}=\quad \mathrm{I} .0004053 \\
& \frac{33}{64} k^{\prime 4}=.0017842 \\
& \frac{\mathrm{I} 85}{\mathrm{I} 536} k^{\prime 6}=\quad .0000245 \\
& \frac{\mathrm{IO} 7}{256} k^{\prime 6}=.000085 \mathrm{I} \\
& \frac{5913}{16384} k^{\prime 8}=.0000042 \\
& \frac{7465}{65536} k^{\prime 8}=.0000012 \\
& \mathrm{I} .00043 \mathrm{IO}=C \\
& \text { Sum }=\mathrm{I} .04599 \mathrm{II}=B \\
& B \log \left(\frac{4}{k^{\prime}}-\mathrm{I}\right)=\mathrm{I} .885^{8184} ;\left\{B \log \left(\frac{4}{k^{\prime}}-\mathrm{I}\right)-C\right\}=0.8853874
\end{aligned}
$$

Multiplying by $4 \pi \sqrt{500}$ gives $M=248.7873 \mathrm{~cm}$, agreeing almost exactly with the value previously found, example 4 . 
EXAMPLE 9. NAGAOKA'S FORMULA (8). CIRCLES NOT NEAR TOGETHER

$$
\begin{aligned}
A & =a=25 \quad d=20 \quad \text { (See Fig. 3.) } \\
\sqrt{k^{\prime}} & =\left(\frac{20}{\sqrt{2900}}\right)^{\frac{1}{2}}=0.6094 \mathrm{I} 83 \\
\frac{l}{2}=\frac{\mathrm{I}-\sqrt{k^{\prime}}}{2\left(\mathrm{I}+\sqrt{k^{\prime}}\right)} & =\frac{\mathrm{I}}{2} \frac{0.39058 \mathrm{I} 7}{\mathrm{I} .6094 \mathrm{I} 83}=0 . \mathrm{I} 2 \mathrm{I} 34250
\end{aligned}
$$

From Table XV, $q-\frac{l}{2}=0.00005269$

$$
\begin{aligned}
\therefore q & =\overline{0.12139519} \\
\frac{3}{2} \log q & =\overline{2} .6263022
\end{aligned}
$$

From Table XV, log $(\mathrm{I}+\epsilon)=0.0002775$

$$
\begin{aligned}
\log 400 \pi^{2} & =3.5963598 \\
\log M & =2.2229395 \\
\therefore M & =167.08577 \mathrm{~cm}
\end{aligned}
$$

or about one in a million higher than the value found for the same circles in example $\mathrm{I}$.

EXAMPLE 10. NAGAOKA'S FORMULA (8). CIRCLES FAR APART

$$
\begin{array}{r}
A=a=\mathrm{IO} \quad d=\mathrm{IOO} \\
k^{\prime}=\frac{\mathrm{IOO}}{\sqrt{\mathrm{IO} 400}}=0.98058073 \\
k=\frac{20}{\sqrt{\mathrm{IO} 400}}=0 . \mathrm{I} 96 \mathrm{II} 6 \mathrm{I} 5 \\
\mathrm{I}+\sqrt{k^{\prime}}=\mathrm{I} .9902427 \\
\frac{l}{2}=\frac{\mathrm{I}}{2} \frac{k^{2}}{\left(\mathrm{I}+k^{\prime}\right)\left(\mathrm{I}+\sqrt{k^{\prime}}\right)^{2}}=0.00245 \mathrm{I} 2756
\end{array}
$$

The differences $q-\frac{l}{2}$ and $\epsilon$ are negligible, so that we have

$$
M=\mathrm{I} 6 \pi^{2} \sqrt{A a}\left(\frac{l}{2}\right)^{\frac{3}{2}}=0.19164966 \mathrm{~cm}
$$


which is in very close agreement with the valves found by formulas (5) and (6) and Havelock's formula (I7) for the same pair of circles. If we calculate $\frac{l}{2}$ by the formula $\frac{\mathrm{I}}{2} \frac{\left(\mathrm{I}-\sqrt{k^{\prime}}\right)}{\left(\mathrm{I}+\sqrt{k^{\prime}}\right)}$ instead we find difficulty in obtaining $\left(\mathrm{I}-\sqrt{k^{\prime}}\right)$ with sufficient precision. The value of $M$ found by using this formula for $\frac{l}{2}$ and with seven place logarithms is in this case $M=0.19164980$, or about one part in a million different.

EXAMPLE 11. NAGAOKA'S SECOND FORMULA (9). FOR CIRCLES NEAR EACH OTHER

$$
\begin{gathered}
A=a=25 \quad d=4 \\
k=\sin \gamma=\frac{50}{\sqrt{25^{\mathrm{I} 6}}}=0.9968 \mathrm{I} 535 \quad \sqrt{k}=0.99840640 \\
k^{\prime 2}=\frac{\sqrt{(A-a)^{2}+d^{2}}}{\sqrt{(A+a)^{2}+d^{2}}}=\frac{4}{\sqrt{25^{\mathrm{I} 6}}}=0.0063593014 \\
\frac{l_{1}}{2}=\frac{k^{\prime 2}}{(\mathrm{I}+k)\left(\mathrm{I}+{\sqrt{k})^{2}}^{2}\right.}=0.00039872542=q_{1} \\
\text { as }\left(\frac{l_{1}}{2}\right)^{5} \text { and higher powers can be neglected. } \\
\log _{e}\left(\frac{\mathrm{I}}{q_{1}}\right)=\log _{e} 2507.9919=7.8272376
\end{gathered}
$$

From Table XVI

$$
-\epsilon_{1}{ }^{\prime}=0.00000128
$$

$$
8 q_{1}+\epsilon_{1}^{\prime}=0.00318852
$$

$$
\begin{aligned}
{\left[\mathrm{I}+8 q_{1}+\epsilon_{1}^{\prime}\right] \log _{e}\left(\frac{\mathrm{I}}{q_{1}}\right)-4 } & =3.8521929=P \\
\frac{\mathrm{I}}{2\left(\mathrm{I}-2 q_{1}\right)^{2}} & =0.50079850=Q \\
\therefore M=4 \pi \sqrt{A a} \cdot P Q & =606.0674 \mathrm{~cm}
\end{aligned}
$$

which is exactly the same value as was found for the same circles in example 5 . 
Using Table II for the above problem, where $\tan \gamma=\mathrm{I} 2.5$, we have $\log F=0.5932708$ and $\log E=0.0047004$. Using these values in formula (I) we obtain for the mutual inductance

$$
M=606.0666 \mathrm{~cm}
$$

which differs from the value by Nagaoka's formula by I part in a million.

EXAMPLE 12. MAXWELL'S SERIES FORMULA (10). FOR ANY TWO COAXIAL CIRCLES NEAR EACH OTHER

$$
A=26 \quad a=25 \quad d=\mathrm{I} \quad c=\mathrm{I} \quad r=\sqrt{2}
$$

$$
\begin{aligned}
& \text { Since } r=\sqrt{2}, \quad \log _{e} \frac{8 a}{r}=\log _{e} \frac{200}{\sqrt{2}}=4.951743^{8} \\
& \mathrm{I}+\frac{c}{2 a}=\mathrm{I} .0200000 \quad 2+\frac{c}{2 a}=2.0200000 \\
& \frac{c^{2}+3 d^{2}}{\mathrm{I} 6 a^{2}}=.0004000 \quad-\frac{3 c^{2}-d^{2}}{\mathrm{I} 6 a^{2}}=-.0002000 \\
& -\frac{c^{3}+3 c d^{2}}{32 a^{3}}=-\frac{.0000080}{1.0203920}=B \quad+\frac{c^{3}-6 c d^{2}}{48 a^{3}}=-\frac{.0000067}{2.0197933}=C \\
& B \log \frac{8 a}{r}=5.0527192 \\
& C=\underline{2.0197933} \\
& \left\{B \log \frac{8 a}{r}-C\right\}=3.0329259 \text { Multiply by } 4 \pi a=\mathrm{IOO} \pi \text { and } \\
& M=952.82 \mathrm{I} 8 \mathrm{~cm} \text {. }
\end{aligned}
$$

This formula would be less accurate for the circles of problem 4 , but is accurate for circles close together, as this problem shows.

EXAMPLE 13. MAXWELL'S FORMULA (12). FOR CIRCLES OF EQUAL RADII NEAR EACH OTHER

$$
\begin{aligned}
A=a=25 \quad d=\mathrm{I} & \\
\frac{8 a}{d}=200 \quad \log _{e} 200 & =5.2983 \mathrm{I} 7 \\
\log _{e} \frac{8 a}{d}\left(\mathrm{I}+\frac{3 d^{2}}{\mathrm{I} 6 a^{2}}\right)=\mathrm{I} .000300 & \times 5.2983 \mathrm{I} 7=5.29990 \\
\left(2+\frac{d^{2}}{\mathrm{I} 6 a^{2}}\right) & =\frac{2.000 \mathrm{IO}}{3.29980} \\
M & =\mathrm{I0} 36.663 \mathrm{~cm}
\end{aligned}
$$

nearly agreeing with the more exact value found under problem 2. 
This is a very simple and convenient formula for equal circles and gives approximate results for circles still farther apart than in this problem.

EXAMPLE 14. HAVELOCK'S FORMULA (16). FOR CIRCLES AT MODERATE DISTANCES

$$
\begin{aligned}
A=25 \quad a=20 \quad d & =\mathrm{IO} \quad \therefore c=5 \\
r=5 \sqrt{5} \quad \alpha=\frac{r^{2}}{A \alpha} & =\frac{\mathrm{I}}{4} \quad \frac{8 \sqrt{A a}}{r}=\mathrm{I} 6 \\
\log _{e} \mathrm{I} 6 & =2.7725887 \\
\mathrm{I}+\frac{3}{\mathrm{I} 6} \alpha & =\mathrm{I.0468750} \\
-\frac{\mathrm{I} 5}{\mathrm{IO} 24} \alpha^{2} & =-0.0009 \mathrm{I} 55 \\
\frac{35}{\mathrm{I} 28^{2}} \alpha^{3} & =0.0000334 \\
-\frac{\mathrm{I} 575}{2 . \mathrm{I} 28^{3}} \alpha^{4} & =-\frac{0.00000 \mathrm{I} 5}{\mathrm{~S} .04599 \mathrm{I} 4} \\
\text { Sum } & =\quad 2.900 \mathrm{IO} 37=B
\end{aligned}
$$

$$
\begin{aligned}
2+\frac{\mathrm{I}}{\mathrm{I} 6} \alpha & =2.0156250 \\
-\frac{3 \mathrm{I}}{2048} \alpha^{2} & =-0.000946 \mathrm{I} \\
\frac{247}{6.128} \alpha^{3} & =0.0000393 \\
-\frac{7795}{8 . \overline{\mathrm{I} 28}{ }^{3}} \alpha^{4} & =-\underline{0.00000 \mathrm{I} 8} \\
\text { Sum } & =2.0147164=C \\
B-C & =0.8853873
\end{aligned}
$$

Multiplied by $4 \pi \sqrt{A a}=248.7873 \mathrm{~cm}=M$

which agrees exactly with the value found in example 8 . $21674^{\circ}-\mathrm{r} 2-3$ 
If the example I 2 be calculated by this formula, no terms of order higher than $\alpha^{2}$ need be calculated, and

$$
M=952.8221 \mathrm{~cm}
$$

Formula (IO) $M=952.82$ I 8

Formula (3) $M=952.8219$

EXAMPLE 15. COFFIN'S FORMULA (13). EXTENSION OF FORMULA (12) FOR CIRCLES OF EQUAL RADII

$$
\begin{aligned}
A=a=25 \quad d & =\mathrm{I} 6 \\
\frac{8 a}{d}=\mathrm{I} 2.5 \quad \log _{e} \mathrm{I} 2.5 & =2.5257286
\end{aligned}
$$

First series of terms $=B=\mathrm{I} .074478$

Second series of terms $=C=2.023220$

$$
\begin{gathered}
\therefore\left\{B \log \frac{8 a}{d}-C\right\}=0.690620 \\
4 \pi a=100 \pi \quad \therefore M=216.9647 \mathrm{~cm} .
\end{gathered}
$$

This agrees with the value given by formula (I) within I part in 200,000. As the distance apart of the circles increases the accuracy by this formula of course gradually decreases.

EXAMPLE 16. FORMULA (14). EXTENSION OF MAXWELL'S FORMULA (10) FOR CIRCLES OF UNEQUAL RADII

$$
\begin{aligned}
A=25 \quad a=20 \quad c=5 \quad d= & \text { I0 } \\
r=\sqrt{c^{2}+d^{2}}=5 \sqrt{5} \quad \log _{e} \frac{8 a}{r}=\log _{e} \frac{32}{\sqrt{5}} & =2.66 \text { I0I69 } \\
\text { First series of terms }=B \log _{e} \frac{8 a}{r} & =3.112060 \\
\text { Second series of terms }=C \quad & =\frac{2.122114}{0.989946} \\
\text { multiplying by } 4 \pi a=80 \pi \quad M & =248.8006 \mathrm{~cm} .
\end{aligned}
$$

This result is correct to I part in 19,000 (see examples 4,8 , and I4). Using only the first three terms for $B$ and $C$ (that is, formula IO), the result would be too large by I part in 1750 .

EXAMPLE 17. HAVELOCK'S FORMULA (17). CIRCLES FAR APART

$$
\begin{array}{rlrl}
a & =\mathrm{IO}=A & d & =\mathrm{IOO} \\
\frac{a}{A} & =\mathrm{I} & \frac{A}{d} & =\mathrm{O} . \mathrm{I}
\end{array}
$$




$$
\begin{aligned}
I-\frac{3}{2} \cdot 2 \cdot \frac{A^{2}}{d^{2}} & =0.9700000 \\
\frac{I 5}{8} \cdot 5 \cdot \frac{A^{4}}{d^{4}} & =0.0009375 \\
-\frac{35}{I 6} \cdot I 4 \cdot \frac{A^{6}}{d^{6}} & =-0.0000306 \\
\frac{3 I 5}{I 28} \cdot 42 \cdot \frac{A^{8}}{d^{8}} & =\frac{0.0000010}{\mathrm{Sum}}=0.9709079
\end{aligned}
$$

Multiplied by $\frac{2 \pi^{2} A^{2} a^{2}}{d^{3}}=0.1916495^{8} \mathrm{~cm}=M$

which is in exact agreement with the value found by formula (6).

EXAMPLE 18. MATHY'S FORMULA (18)

\begin{tabular}{|c|c|c|c|}
\hline I.0022006 & I.OII 2962 & 0.9969192 & I.0095357 \\
\hline 0.0000357 & 0.0002173 & -0.0000473 & 0.000I784 \\
\hline 0.0000008 & 0.0000049 & - o.0000010 & 0.0000040 \\
\hline$\overline{1.002237 I}$ & $\overline{\text { I.OII5I } 84}$ & 0.9968709 & 1.009718 \\
\hline
\end{tabular}

$$
\begin{aligned}
& A=25 \quad a=20 \quad d=40 \\
& x^{2}=625+400+\mathrm{r} 600=2625 \\
& \frac{A a}{x^{2}}=\frac{500}{5625}=\frac{4}{2 \mathrm{I}} \\
& x^{4}+12 A^{2} a^{2}=9890625 \\
& \frac{\mathrm{I}}{4} \log \left(x^{4}+\mathrm{I2} A^{2} \alpha^{2}\right)=\mathrm{I} .7488059 \\
& \log \left[\frac{x^{2}}{\left(x^{4}+12 A^{2} a^{2}\right)^{\frac{1}{4}}}\right]=1.6703234 \\
& \mathrm{I}-36 \frac{A^{2} a^{2}}{x^{4}}=-0.306 \mathrm{r} 225 \\
& \mathrm{I}+\mathrm{I} 2 \frac{A^{2} a^{2}}{x^{4}}=\mathrm{I} .4353742
\end{aligned}
$$

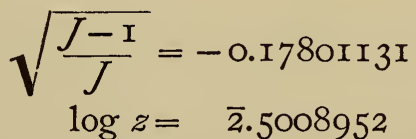

Using the constants in Table XVII we calculate the four series

$$
F\left(\frac{\mathrm{I}}{\mathrm{I} 2}, \frac{5}{\mathrm{I} 2}, \frac{\mathrm{I}}{2}, z\right) F\left(\frac{7}{\mathrm{I} 2}, \frac{\mathrm{II}}{\mathrm{I} 2}, \frac{3}{2}, z\right) \quad F\left(-\frac{\mathrm{I}}{\mathrm{I} 2}, \frac{7}{\mathrm{I} 2}, \frac{\mathrm{I}}{2}, z\right) F\left(\frac{5}{\mathrm{I} 2}, \frac{\mathrm{I}}{\mathrm{I} 2}, \frac{3}{2}, z\right)
$$


From these, using the values of the constants already calculated, we find the four terms in the formula for $M$

$$
\begin{array}{rrr}
C= & 26.981438 & G=25.447327 \\
D=-0.639427 & H=-0.9662 \mathrm{II} \\
C-D=\frac{H}{27.620865} & G-\bar{H}=24.48 \mathrm{III2} \\
\frac{M}{4 \pi}=27.620865-24.48 \mathrm{III} 2=3 . \mathrm{I} 39753
\end{array}
$$

By Nagaoka's formula (8) we find $\frac{M}{4 \pi}=3 \cdot 1397496$

$$
\text { " “ (6) “ “ } \frac{M}{4 \pi}=3 \cdot \text { I } 397486
$$

Mathy's formula suffers here under the inconvenience that $M$ is given as the difference of two quantities considerably larger than itself.

EXAMPLE 19. FORMULA (19). FOR CIRCLES SATISFYING THE CONDI-

$$
\begin{gathered}
\text { TION } \mathrm{r}_{1}{ }^{2}=2 \mathrm{r}_{2}{ }^{2} \text { OR } \mathrm{k}^{\prime}=\mathrm{k}=\frac{1}{\sqrt{2}} \\
A=a=25 \quad d=50 \\
M=\mathrm{I} .4 \mathrm{I} 859262 \cdots \cdots \sqrt{A a} \\
=35.46498 \mathrm{I} 6 \cdots \cdots \mathrm{cm} . \\
\text { By Nagaoka's formula (8), } M=35.464975
\end{gathered}
$$

$\begin{array}{lll}\text { " } & \text { " } \quad(6), M=35.46498 \mathrm{I} \\ & \text { " } \quad(\mathrm{I}), M=35.4648 \mathrm{I}\end{array}$

We see that the formulas (8) and (6) here give an accuracy limited only by that of the logarithm tables. The result found by formula (I), using Table XIII, is, however, affected by the fact that the value of the quantity in the parentheses (I.4239I67-I.3IIO287), is only about a tenth as large as the numbers of which it is the difference. 


\section{MUTUAL INDUCTANCE OF TWO COAXIAL COILS}

\section{R.OWLAND'S FORMULA}

Let there be two coaxial coils of mean radii $A$ and $a$, axial breadth of coils $b_{1}$ and $b_{2}$, radial depth $c_{1}$ and $c_{2}$, and distance apart of their mean planes $d$. Suppose them uniformly wound with $n_{1}$ and $n_{2}$ turns of wire. The mutual inductance $M_{0}$ of the two central turns of the coils (Fig. 4), will be given by formula (I) or (7), or any one of the foregoing formulas for the mutual inductance of coaxial circles adapted to the particular case may be used, and the mutual inductance $M$ of the two coils of $n_{1}$ and $n_{2}$ turns will then be, to a first approximation,

$$
M=n_{1} n_{2} M_{0}
$$

The following second approximation was obtained by Rowland by means of Taylor's theorem, following Maxwell, $\S 700$ :

$$
\begin{aligned}
\frac{M}{n_{1} n_{2}} & =M_{0}+\frac{I}{24}\left\{\left(b_{1}{ }^{2}+b_{2}{ }^{2}\right) \frac{d M_{0}}{d x^{2}}\right. \\
& \left.+c_{1}{ }^{2} \frac{d^{2} M_{0}}{d a^{2}}+c_{2}{ }^{2} \frac{d^{2} M_{0}}{d A^{2}}\right\}
\end{aligned}
$$

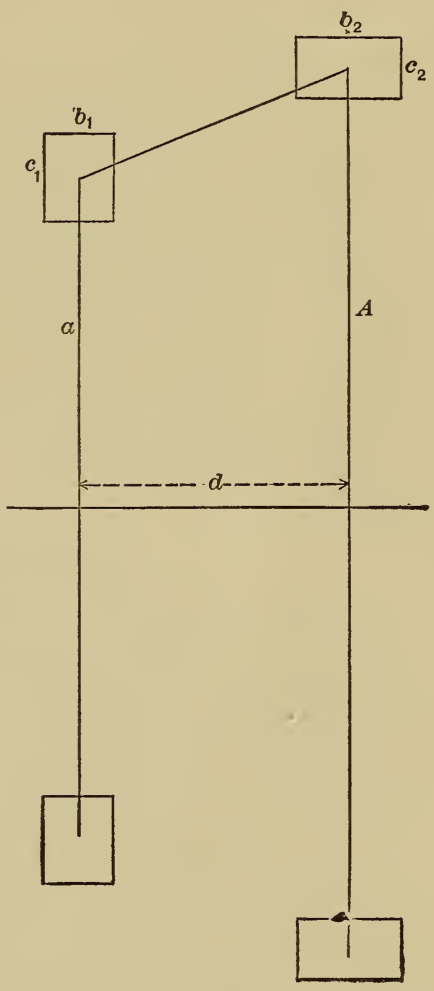

Fig. 4

If the two coils are of equal radii but unequal section,

$$
\frac{M}{n_{1} n_{2}}=M_{0}+\frac{\mathrm{I}}{24}\left\{\left(b_{1}{ }^{2}+b_{2}^{2}\right) \frac{d^{2} M_{0}}{d x^{2}}+\left(c_{1}^{2}+c_{2}^{2}\right) \frac{d^{2} M_{0}}{d a^{2}}\right\}
$$

If the two coils are of equal radii and equal section, this becomes

$$
\frac{M}{n_{1} n_{2}}=M_{0}+\frac{\mathrm{I}}{\mathrm{I} 2}\left\{b^{2} \frac{d^{2} M_{0}}{d x^{2}}+c^{2} \frac{d^{2} M_{0}}{d a^{2}}\right\}
$$


The correction terms will be calculated by means of the following:

$$
\begin{aligned}
& \frac{d^{2} M_{0}}{d a^{2}}=\pi \frac{k}{a}\left\{\left(2-k^{2}\right) F-\left(2-k^{2} \frac{I-2 k^{2}}{\mathrm{I}-k^{2}}\right) E\right\} \\
& \frac{d^{2} M_{0}}{d x^{2}}=\pi \frac{k^{3}}{a}\left\{F-\frac{\mathrm{I}-2 k^{2}}{\mathrm{I}-k^{2}} E\right\}
\end{aligned}
$$

The equation (2I) is equivalent to Rowland's equation, where $2 \xi$ and $2 \eta$ are the breadth and depth of the section of the coil, instead of $b$ and $c$, except that there is an error in the formula as printed in Rowland's ${ }^{16}$ paper, $\xi$ and $\eta$ being interchanged. The equations (22) are equivalent to those given by Rowland, being somewhat simpler. ${ }^{17}$

Formula (2I) gives a very exact value for the mutual inductance of two coils, provided the cross sections are relatively small and the distance apart $d$ is not too small. But when $b$ or $c$ is large or $d$ is small the fourth differential coefficients which have been neglected become appreciable and the expression may not be sufficiently exact.

\section{RAYLEIGH'S FORMULA}

Maxwell ${ }^{18}$ gives a formula, suggested by Rayleigh, for the mutual inductance of two coils, which has a very different form from Rowland's, but is nearly equivalent to it when the coils are not near each other. It has been used by Rayleigh in calculating the mutual inductance of a Lorenz apparatus and by Glazebrook (Phil. Trans., I883) in calculating the mutual inductance of parallel coils of rectangular section employed in a determination of the ohm. It may also be employed in calculating the attraction between two coils. ${ }^{19}$ It is sometimes called the formula of quadratures, and is as follows: ${ }^{20}$

$$
M=\frac{I}{6}\left(M_{1}+M_{2}+M_{3}+M_{4}+M_{5}+M_{6}+M_{7}+M_{8}-2 M_{0}\right)
$$

${ }^{16}$ Collected Papers, p. I62. Am. Jour. Sci. [3], XV, I878.

${ }^{17}$ Gray, Absolute Measurements, Vol. II, Part II, p. 322.

${ }^{18}$ Electricity and Magnetism, Vol. II, Appendix II, Chapter XIV.

${ }^{19}$ Gray, Absolute Measurements, Vol. II, Part II, p. 403.

${ }^{20}$ This Bulletin, 2, p. 370-372; I906. 
where $M_{1}$ is the mutual inductance of the circle $\mathrm{O}_{2}$ and a circle through the point I of radius $A-\frac{c_{1}}{2}$, and similarly for the others, Fig. 5.

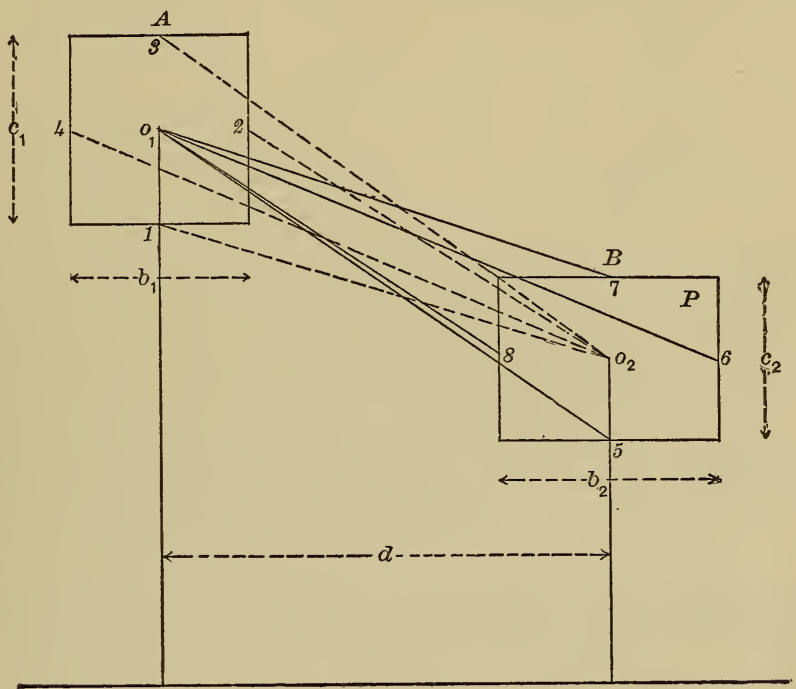

Fig. 5

For two coils of equal radii and equal section this becomes

$$
M=\frac{\mathrm{I}}{3}\left(M_{1}+M_{2}+M_{3}+M_{4}-M_{0}\right)
$$

Equation (23) is Rayleigh's formula, or the formula of quadratures. Instead of computing the correction to $M_{0}$ by means of the differential coefficients (2O), eight additional values are computed, corresponding to the mutual inductances of the single turns at the eight numbered points indicated in Fig. 5, each with reference to the central turn of the other coil. These $M^{\prime}$ 's may be computed by any of the formulas for the mutual inductance of coaxial circles which may be best adapted to the particular case, and the values of the constants for the case of two coils of equal radii are given in the following table, the radius being $a$ in every case. 


\section{Axial distance}

Using (IO)

《

《

《

Using (I 2)

"

"

《 $d$

$d$

$d$

$d$

$d-b_{1} / 2$

$d+b_{1} / 2$

$d+b_{2} / 2$

$d-b_{2} / 2$
Radial distance

$-\frac{c_{1}}{2}$

$+\frac{c_{1}}{2}$

$-\frac{c_{2}}{2}$

$+\frac{c_{2}}{2}$

$\mathrm{O}$

$\mathrm{O}$

$\mathrm{O}$

$\mathrm{O}$

\section{MAGNITUDE OF THE ERRORS IN ROWLAND'S AND RAYLEIGH'S FORMULAS}

The error $\epsilon_{1}$ in equation (24), for two coils of equal radii $a$, distance between centers being $d$, and section $b \times c$ (Fig. 6), depends on the dimensions of the coil in a manner shown by the following expression: ${ }^{21}$

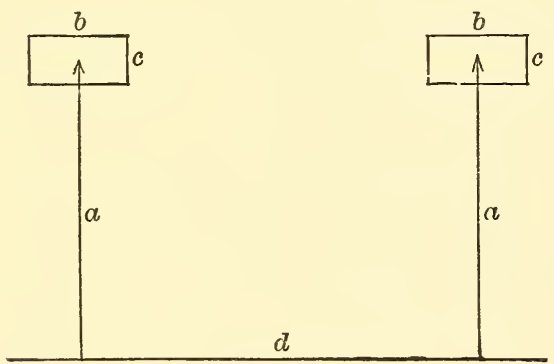

Fig. 6

$$
\epsilon_{1} \propto 4 \pi a\left\{\frac{3 b^{4}+3 c^{4}-20 b^{2} c^{2}}{480 d^{4}}\right\}
$$

For a square coil the correction is a negative quantity, showing that $M$ by equation (24) is too large, and the error is proportional to the fourth power of $\frac{I}{d}$, the reciprocal of the

distance between the mean planes of the coils. For a rectangular coil in which $b$ is greater than $c$ the correction is negative so long as $b$ is not more than 2.5 times $c$. When $b$ is still larger with respect to $c$ the correction becomes plus, the value of $M$ by (24) being too small. 
Thus, for a coil of cross section 4 sq. cm, we get the following values of the numerator of (25) as we vary the shape of cross section keeping $b c=4$.

$\begin{array}{ccc}\text { Dimensions of coil } & \text { Error proportional to- } \\ b=2 & c=2 & -224 \\ b=2.5 & c=\mathrm{I} .6 & -\mathrm{I} 83 \\ b=3 & c=\mathrm{I} .33 & -67.5 \\ b=4 & c=\mathrm{I} & +45 \mathrm{I} \\ b=8 & c=0.5 & +\mathrm{II}, 988\end{array}$

Thus we see that the value of $M$ as given by the formula of quadratures may be too large or too small according to the shape of the section, and that the error is proportional directly to the fourth power of the dimensions of the section and inversely to the fourth power of the distance between the mean planes of the coils. When the section is small and $d$ large the error will become negligible.

The error by Rowland's formula is- ${ }^{22}$

$$
\epsilon_{2} \propto 4 \pi a \frac{6}{d^{4}}\left\{\frac{b^{4}+c^{4}}{360}-\frac{b^{2} c^{2}}{I_{44}}\right\} \propto 4 \pi a\left\{\frac{8 b^{4}+8 c^{4}-20 b^{2} c^{2}}{480 d^{4}}\right\}
$$

This is negative for a square coil, but smaller than $\epsilon_{1}$. For a coil of section such that $b=c \sqrt{2}$, the error is zero, and for sections such that $\frac{b}{c}>\sqrt{2}$, the error is positive. Thus, for a coil of cross section 4 sq. cm, we get the following values of the numerator of (26) which is proportional to the error by Rowland's formula.

$$
\begin{array}{ll}
\text { Dimensions of coil } \\
b=2 & c=2 \\
b=2.5 & c=\mathrm{I} .6 \\
b=3 & c=\mathrm{I} .33 \\
b=4 & c=\mathrm{I} \\
b=8 & c=0.5
\end{array}
$$

Error proportional to-

$$
\begin{array}{rr}
-\quad 64 \\
+\quad 45 \\
+\quad 353 \\
+\quad 1,736 \\
+32,448
\end{array}
$$

Thus the error is smaller by Rowland's formula for coils having square or nearly square section, but larger for coils having rectangular sections not nearly square. 


\section{LYLE'S FORMULA}

Professor Lyle ${ }^{23}$ has recently proposed a very convenient method for calculating the mutual inductance of coaxial coils, which gives very accurate results for coils at some distance from each other.

The mutual inductance is calculated from formula (I) or any other formula for two coaxial circles, using, however, a modified radius $r$ instead of the mean radius $a, r$ being given by the following equation when the section is square, $b$ being the side of the square section :

$$
r=a\left(\mathrm{I}+\frac{b^{2}}{24 a^{2}}\right)
$$

If the coil has a rectangular section not square, it can be replaced by two filaments (Fig. 7) the distance apart of the filaments being called the equivalent breadth or the equivalent depth of the coil.

$$
\begin{aligned}
& \beta^{2}=\frac{b^{2}-c^{2}}{\mathrm{I} 2}, 2 \beta \text { is the equivalent breadth of } \mathrm{A} \\
& \delta^{2}=\frac{c^{2}-b^{2}}{\mathrm{I} 2}, 2 \delta \text { is the equivalent depth of } \mathrm{B}
\end{aligned}
$$

The equivalent radius of $\mathrm{A}$ is given by the same expression which holds for a square coil, viz:

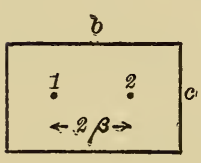

$A$

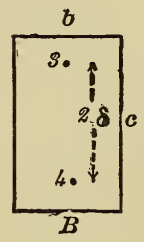

Fig 7.

$$
r=a\left(\mathrm{I}+\frac{c^{2}}{24 a^{2}}\right)
$$

In the coil $\mathrm{B}$ the equivalent filaments have radii $r+\delta$ and $r-\delta$, respectively, where

$$
r=a\left(\mathrm{I}+\frac{b^{2}}{24 a^{2}}\right)
$$

The mutual inductance of two coils may now be readily calculated. If each has a square section, it is necessary only to calculate the mutual inductance of the two equivalent filaments. For coils of rectangular sections, as A, B, the mutual inductance will be the sum of the mutual inductances of the two filaments of $A$ on the two

${ }^{23}$ Phil. Mag., 3, p. 3 Io; Igo2. Also this Bulletin, 2, pp. 374-378; 1906. 
filaments of $\mathrm{B}$, counting $n / 2$ turns in each. Or, it is $n_{1} n_{2}$ times the mean of the four inductances $M_{13}, M_{14}, M_{23}, M_{24}$, where $M_{13}$ is the mutual inductance of filament I on filament 3 , etc.

Lyle's method is of special value in computing mutual inductances because it applies to coils of unequal as well as of equal radii.

\section{ROSA'S FORMULA ${ }^{24}$}

Writing the mutual inductance of two coaxial coils of equal radii and equal section as $\frac{M}{n_{1} n_{2}}=M_{0}+\Delta M$, where $M_{0}$ is the mutual inductance of the central circles of the two equal coils of sections $b \times c$, Fig. 5 , and $\Delta M$ is the correction for the section of the coil, the value of $\Delta M$ is as follows:

$$
\begin{gathered}
\Delta M=4 \pi a\left\{\frac{3 b^{2}+c^{2}}{96 a^{2}} \cdot \log \frac{8 a}{d}-\frac{\mathrm{II} b^{2}-3 c^{2}}{\mathrm{I} 92 a^{2}}+\frac{b^{2}-c^{2}}{\mathrm{I} 2 d^{2}}+\frac{2 b^{4}+2 c^{4}-5 b^{2} c^{2}}{\mathrm{I} 20 d^{4}}\right. \\
+\frac{6 b^{4}+6 c^{4}+5 b^{2} c^{2}}{5760 a^{2} d^{2}}+\frac{3 b^{6}-3 c^{6}+\mathrm{I} 4 b^{2} c^{4}-\mathrm{I} 4 b^{4} c^{2}}{504 d^{6}}+\frac{7 c^{2} d^{2}}{\mathrm{IO} 24 a^{4}}\left(\log \frac{8 a}{d}-\frac{\mathrm{I} 63}{84}\right) \\
\left.-\frac{\mathrm{I} 5 b^{2} d^{2}}{\mathrm{IO} 24 a^{4}}\left(\log \frac{8 a}{d}-\frac{97}{60}\right)\right\}
\end{gathered}
$$

For a square section, when $b=c$, this becomes

$$
\Delta M=\frac{\pi b^{2}}{6 a}\left\{\log \frac{8 a}{d}-\mathrm{I}-\frac{a^{2} b^{2}}{5 d^{4}}-\frac{3 d^{2}}{16 a^{2}}\left(\log \frac{8 a}{d}-\frac{4}{3}\right)+\frac{\mathrm{I} 7 b^{2}}{240 d^{2}}\right\} \quad \text { [30] }
$$

The last two terms of equation (30) are relatively small, so that we may write, approximately:

$$
\Delta M=\frac{\pi b^{2}}{6 a}\left\{\log \frac{8 a}{d}-\mathrm{I}-\frac{a^{2} b^{2}}{5 d^{4}}\right\}
$$

For coils of equal radii but unequal sections, the formula is, neglecting differentials of sixth order

$$
\begin{aligned}
& \Delta M=4 \pi a\left\{\frac{3\left(b_{1}^{2}+b_{2}^{2}\right)+\left(c_{1}^{2}+c_{2}^{2}\right)}{\operatorname{Ig} 2 a^{2}} \log \frac{8 a^{d}}{d}-\frac{\operatorname{II}\left(b_{1}{ }^{2}+b_{2}{ }^{2}\right)-3\left(c_{1}^{2}+c_{2}^{2}\right)}{384 a^{2}}\right. \\
& +\frac{\left(b_{1}^{2}+b_{2}^{2}\right)-\left(c_{1}^{2}+c_{2}^{2}\right)}{24 d^{2}} \\
& \left.+\frac{\left(3 b_{1}^{4}+\operatorname{IO}_{1}{ }^{2} b_{2}{ }^{2}+3 b_{2}{ }^{4}\right)+\left(3 c_{1}^{4}+\operatorname{IO}_{1}{ }^{2} c_{2}{ }^{2}+3 c_{2}^{4}\right)-\operatorname{IO}\left(b_{1}{ }^{2}+b_{2}{ }^{2}\right)\left(c_{1}{ }^{2}+c_{2}{ }^{2}\right)}{960 d^{4}}\right\}
\end{aligned}
$$

${ }^{24}$ This Bulletin, 4, p. 348 , equations (38) and (39). 
These expressions for $\Delta M$ are very exact where the coils are near together or even where they are separated by a considerable distance, but become less exact as $d$ is greater. They are therefore most reliable where formulas (2I), (24), and (27) are least reliable. As formula (3I) is exact enough for most purposes, it affords a very easy method of getting the correction for equal coils of square section.

Stefan's formula for the mutual inductance of two equal coaxial coils (originally published ${ }^{25}$ without demonstration) is incorrect and is not given here. It resembles equation (29), but is seriously in error for coils at considerable distances.

\section{THE ROSA-WEINSTEIN FORMULA}

Weinstein's formula ${ }^{26}$ for the mutual inductance of equal coaxial coils has been revised and corrected by Rosa, and the value of $\Delta M$, the correction for section, expressed separately. The expression for $\Delta M$ is as follows: ${ }^{26 a}$

$$
\Delta M=4 \pi \alpha \sin \gamma\left\{(F-E)\left(A+\frac{c^{2}}{24 \alpha^{2}}\right)+E B\right\}
$$

where $F$ and $E$ are the complete elliptic integrals to modulus $\sin \gamma$, Fig. 8 (as in equation I),

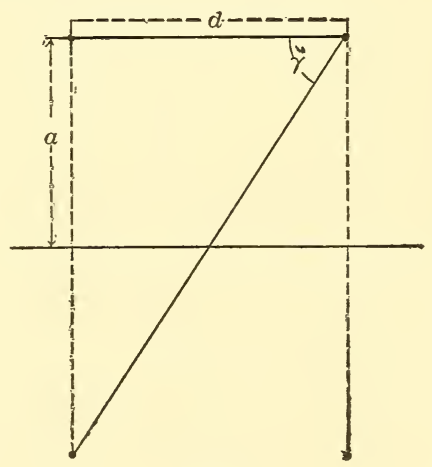

Fig. 8

${ }^{25}$ Wied. Annalen, 22, p. I07; I884.

${ }^{26}$ Wied. Annalen, 21, p. 350; I884.

${ }^{26 a}$ This Bulletin, 4, p. 342, equation (20); 1907. 
and

$$
\begin{aligned}
& A=\frac{\cos ^{2} \gamma}{\mathrm{I} 2 d^{2}}\left(\alpha_{1}-\alpha_{2}-\alpha_{3}+\left(2 \alpha_{2}-3 \alpha_{3}\right) \cos ^{2} \gamma+8 \alpha_{3} \cos ^{4} \gamma\right) \\
& B=\frac{\sin ^{2} \gamma}{\mathrm{I} 2 d^{2}}\left(\alpha_{1}+\frac{\alpha_{2}}{2}+2 \alpha_{3}+\left(2 \alpha_{2}+3 \alpha_{3}\right) \cos ^{2} \gamma+8 \alpha_{3} \cos ^{4} \gamma\right)
\end{aligned}
$$

The values of $\alpha_{1}, \alpha_{2}$, and $\alpha_{3}$ are as follows:

$$
\begin{aligned}
& \alpha_{1}=b^{2}-c^{2}+\frac{c^{4}}{30 a^{2}} \quad \text { For square section: } \alpha_{1}=\frac{b^{4}}{30 a^{2}} \\
& \alpha_{2}=\frac{5 b^{2} c^{2}-4 c^{4}}{60 a^{2}} \quad \text { " } \quad \text { " } \quad \alpha_{2}=\frac{b^{4}}{60 a^{2}} \\
& \alpha_{3}=\frac{2 b^{4}+2 c^{4}-5 b^{2} c^{2}}{20 d^{2}} \quad \text { " } \quad \text { " } \quad \alpha_{3}=-\frac{b^{4}}{20 d^{2}}
\end{aligned}
$$

Formula (33) is a very exact formula for all positions of the two coils, except when they are very close together.

Weinstein's original formula, ${ }^{27}$ which is much less accurate than (33) for coils relatively near together, is not here given.

\section{USE OF FORMULAS FOR SELF-INDUCTANCE IN CALCULATING MUTUAL}

\section{INDUCTANCE}

One can sometimes obtain the mutual inductance of adjacent coils, or of coils at a distance from one another, by means of a formula for the self-inductance of coils. Thus, suppose we have a coil of rectangular section, which we subdivide into three equal parts, I, 2, 3, Fig. 9. Let $L$ be the self-inductance of the whole coil, $L_{1}$ be the self-inductance of any one of the three equal smaller coils, and $L_{2}$ be the selfinductance of two adjacent coils taken together. Also let $M_{12}$ be the mutual inductance of coil I on coil 2 , or of coil 2 on coil 3 , and $M_{13}$ be the mutual inductance of coil I on coil 3. Then,

$$
\begin{aligned}
L_{1} & =3 L_{1}+4 M_{12}+2 M_{13} \\
\text { Also, } L_{2} & =2 L_{1}+2 M_{12} \\
\therefore M_{12} & =\frac{L_{2}-2 L_{1}}{2} \\
\text { and } M_{13} & =\frac{L+L_{1}-2 L_{2}}{2}
\end{aligned}
$$

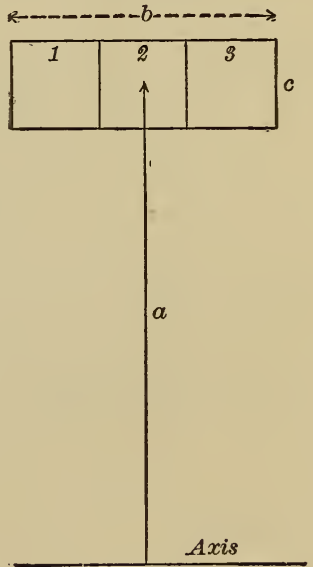

Fig. 9 
Formula (34) will thus enable us to find the mutual inductance of two coils of equal radii adjacent or near each other by the calculation of self-inductances from such formulas as those of Weinstein (88) and Stefan (90). These latter formulas are not, however, exact enough when the section is large to permit us to apply them to coils at any considerable distance from one another.

\section{GEOMETRIC MEAN DISTANCE FORMULA}

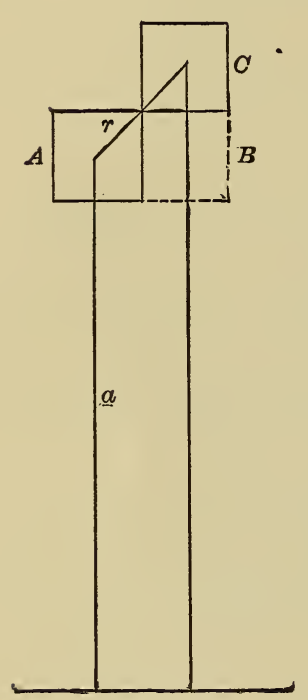

Fig. 10

The mutual induictance of two coaxial coils adjacent or very near can sometimes be obtained by means of the geometric mean distances. This method is accurate only when the sections are very small relatively to the radius. It can often be used to advantage in testing other formulas, but not often in determining the mutual inductance of actual coils.

Formula (IO) gives the mutual inductance of two very near coaxial coils in terms of the geometric mean distance, if $r$ be replaced by $R$, the geometric mean distance of the two sections. Formula (IO) gives $M_{0}$ if $r$ be used, where $r$ is the distance between centers. Thus,

$$
\Delta M=4 \pi a\left(\mathrm{I}+\frac{c}{2 a}\right) \log \frac{r}{R}
$$

For coils $\mathrm{A}$ and $\mathrm{C}$ (Fig. Io), $R<r$ and $\Delta M$ is positive; $R=0.99770 r$ " " A " $\mathrm{B}, R>r$ and $\Delta M$ is negative; $R=1.00655 r$

The same formula may also be used for squares not adjacent, but only when quite near. ${ }^{28}$

For illustrations and tests of the above formulas, see examples 20-33, pages 44-52.

${ }^{28}$ For other values of the geometric mean distances of squares in a plane see this Bulletin, 3, p. I; I907. 


\section{CHOICE OF FORMULAS}

(a) For coils of equal radii and equal cross section (29) should be used if the coils are rather near together. If the cross section is square (29) takes the more simple form (30), and in some cases this may be used in its abbreviated form (3I). For coils at all distances, except near together, (33) gives very good precision; (24) and (2I) are not so accurate as this last, but give good results if the coils are far apart and their cross sections are not too large.

(b) For coils of equal radii but unequal section (32) is accurate for coils not too far away from one another. For coils farther separated (20), (23) or (28) may be used.

(c) For coils of unequal radii (23), (24), (27), and (28) apply, but unfortunately they are not as accurate as some of the others. except when the coils are relatively distant or have very small cross sections. The difficulty can be overcome by subdividing each of the two coils into two, four, or more equal parts, and taking the sum of the mutual inductances of all of the parts of one on all the parts of the other. This is a laborious operation, but in important cases it should be done. As the subdivision is carried further the results will approach a final value,

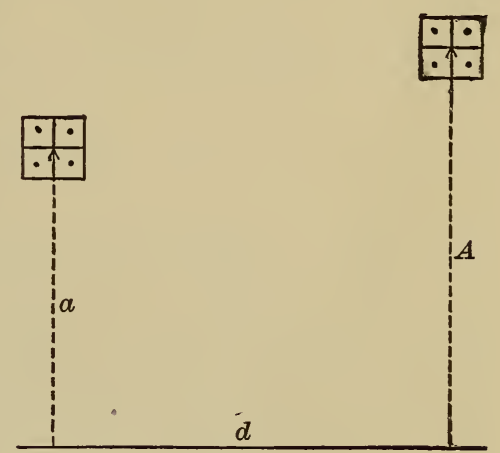

Fig. 11 and hence the results themselves show when the subdivision has been carried far enough.

Thus, suppose two coils A, B (Fig. II) of square section are subdivided into four equal parts and by the method of Lyle, formula (27), the mutual inductance of the whole of $B$ is computed on each of the four parts of $A$. If the sum differs appreciably from the result obtained by taking $A$ and $B$ as wholes in one calculation, then the four parts of B may be taken separately with respect to the separate parts of A. If one is doubtful whether this is sufficiently accurate, one of the sections of A may be subdivided further and calculated with respect to one section of $B$, to see whether there is any appreciable 
difference due to this further subdivision. For coils of equal radii very accurate results for near coils can be obtained much more easily by using some of the other formulas.

\section{EXAMPLES TO ILLUSTRATE THE FORMULAS FOR THE MUTUAL INDUCTANCE OF COILS OF RECTANGULAR SECTION}

EXAMPLE 20. ROWLAND'S FORMULA (21). FOR COAXIAL COILS OF EQUAL RADII

$$
A=a=25 \quad b=c=2 \mathrm{~cm} \quad d=\mathrm{IO}
$$

The mutual inductance of the two coils is $\frac{M}{n_{1} n_{2}}=M_{0}+\Delta M$.

We find $M_{0}$ by formula I, 8, or I 3 , and $\Delta M$ by $2 \mathrm{I}$ and 22.

$$
M_{0}=\mathrm{I0} 7 \cdot 4885 \pi
$$

$$
\begin{aligned}
k=\sin \gamma=\frac{50}{\sqrt{2600}} & =0.9805807 \\
k^{2} & =0.9615383 \\
\log _{10} F & =0.4821754 \\
\log _{10} E & =0.0207625
\end{aligned}
$$

By Table II, since $\tan \gamma=5, \log F=0.4821752$ and $\log E=$ 0.0207626 . These slight differences in the logarithms obtained in the two different ways amount to scarcely one part in two million of $F$ and $E$, respectively, and may usually be neglected. If more accurate values are required they may be obtained by carrying the interpolations further in Legendre's table, provided the angle $\gamma$ is obtained with sufficient accuracy.

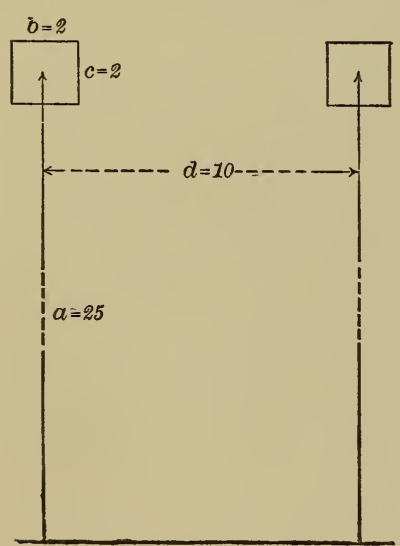

Fig. 12

Substituting these values in formula (22) we obtain

$$
\begin{aligned}
& \frac{d^{2} M}{d a^{2}}=-0.908 \mathrm{I} \pi \\
& \frac{d^{2} M}{d x^{2}}=+\mathrm{I} .0639 \pi \quad b^{2}=c^{2}=4
\end{aligned}
$$

Substituting these values in formula (2I) we obtain

$$
\begin{aligned}
\Delta M & =.05194 \pi \\
\therefore \frac{M}{n_{1} n_{2}}=M_{0}+\Delta M & =(107.4885+0.05 \mathrm{I} 9) \pi \\
& =337.848 \mathrm{I} \mathrm{cm} .
\end{aligned}
$$


The correction $\Delta M$ thus amounts to about I part in 2000 of $M$. At a distance $d=20 \mathrm{~cm}$, the correction is over I part in Iooo. For a coil of section $4 \times 4 \mathrm{~cm}$ at $d=10, \Delta M$ would be four times as large as the value above, or about one part in five hundred, and at $20 \mathrm{~cm}$ one part in two hundred and fifty.

EXAMPLE 21. ROWLAND'S FORMULA (20). FOR COILS OF EQUAL RADII BUT UNEQUAL SECTION

Let us take $a=25, d=$ Io as in the preceding example, but instead of supposing the sections of the coils to be equal let us take

$$
\begin{array}{ll}
b_{1}=4 & b_{2}=2 \\
c_{1}=I & c_{2}=2
\end{array}
$$

The values of $\frac{d^{2} M_{0}}{d x^{2}}$ and $\frac{d^{2} M_{0}}{d a^{2}}$ will be the same as in the preceding example. Substituting these in (20) we find $\Delta M=0.6974 \pi$

$$
\begin{aligned}
M_{0} & =107.4885 \pi \\
\Delta M & =\frac{0.6974 \pi}{\therefore \frac{M}{n_{1} n_{2}}}=\frac{108 . \mathrm{I} 859 \pi}{}=339.876 \mathrm{I} \mathrm{cm} .
\end{aligned}
$$

The correction here is fourteen times as great as in the previous example, although the areas of the cross sections of the two coils are the same as in the preceding case.

EXAMPLE 22. RAYLEIGH'S FORMULA (24). FOR COAXIAL COILS OF EQUAL RADII

$$
A=a=25 \quad b=4 \quad c=\mathrm{I} \quad d=\text { IO }
$$

We now find by formula (I) in accordance with formula (24) the mutual inductance of the following pairs of circles (Fig. I3):

$\mathrm{O}$, I when $a=25, A=25.5, d=10 ; \mathrm{O}, 4$ when $a=25, A=24.5$, $d=\mathrm{IO} ; \mathrm{O}, 2$ when $a=A=25$ and $d=8 ; \mathrm{O}, 3$ when $A=a=25, d=\mathrm{I} 2$ and finally $\mathrm{O}, \mathrm{O}^{\prime}$ when $A=a=25, d=$ IO. Thus:

$$
21674^{\circ}-12-4
$$




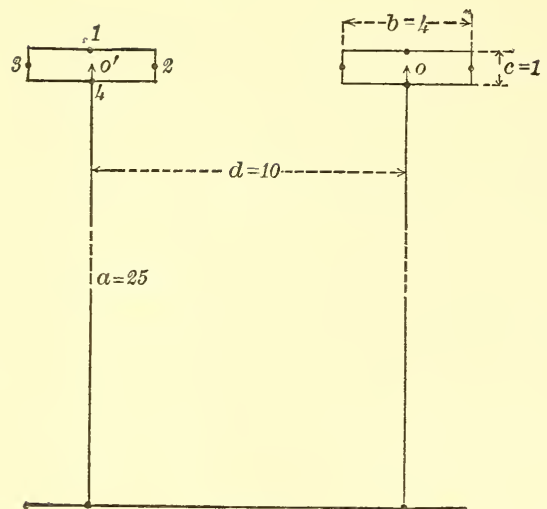

Fig. 13

$$
\begin{aligned}
M_{1} & =\mathrm{IO} 0.32 \mathrm{I} 7 \pi \\
M_{4} & =\mathrm{IO} 5.4287 \pi \\
M_{2} & =\mathrm{I} 27.3949 \pi \\
M_{3} & =\frac{9 \mathrm{I} .9206 \pi}{434.0659 \pi} \\
M_{0} & =\frac{\mathrm{IO} 7.4885 \pi}{326.5774 \pi} \\
\therefore M & =\mathrm{IO} 8.859 \mathrm{I} \pi \\
M_{0} & =\frac{\mathrm{IO} 7.4885 \pi}{\mathrm{I} .3706 \pi} \mathrm{cm} . \\
\Delta M & =
\end{aligned}
$$

EXAMPLE 23. RAYLEIGH'S FORMULA (23). COILS OF UNEQUAL RADI Let

\section{AND UNEQUAL SECTION}

$$
\begin{aligned}
& A=25 \quad b_{1}=4 \quad c_{1}=\mathrm{I} \quad d=\mathrm{IO} \\
& a=20 \quad b_{2}=2 \quad c_{2}=3
\end{aligned}
$$

We have then to calculate the mutual inductances of the following pairs of circles:

\begin{tabular}{rllrlllr} 
& \multicolumn{1}{c}{$A$} & $a$ & $d$ & & $A$ & $a$ & $d$ \\
$M_{1}$ & 24.5 & 20 & IO & $M_{6}$ & 25 & 20 & II \\
$M_{2}$ & 25 & 20 & 8 & $M_{7}$ & 25 & $2 \mathrm{I} .5$ & IO \\
$M_{3}$ & 25.5 & 20 & IO & $M_{8}$ & 25 & 20 & 9 \\
$M_{4}$ & 25 & 20 & I 2 & $M_{0}$ & 25 & 20 & IO \\
$M_{5}$ & 25 & I8.5 & IO & & & &
\end{tabular}

These have been calculated by means of Havelock's formula (I6), with the following results:

$$
\begin{aligned}
M_{1} & =248.4 \mathrm{I} 280 \\
M_{2} & =28, .04 \mathrm{O} 27 \\
M_{3} & =r 8.77440 \quad M_{0}=248.7873 \\
M_{4} & =2 \mathrm{I} 4.75755 \\
M_{5} & =2 \mathrm{I} 6.60 \mathrm{I} 85 \\
M_{6} & =23 \mathrm{I} .04386 \\
M_{7} & =279.8 \mathrm{I} 4 \mathrm{I} 7 \\
M_{8} & =268.094 \mathrm{IO} \\
\mathrm{Sum} & =\frac{\mathrm{I} 996.5390}{2 \mathrm{M}}=497.5746 \\
\mathrm{Diff} & =\mathrm{I} 498.9644 \\
\frac{\mathrm{I}}{6} \mathrm{Diff.} & =249.8272=\frac{M}{n_{1} n_{2}}
\end{aligned}
$$


EXAMPLE 24. LYLE'S FORMULA (27). FOR COLLS OF SQUARE SECTION

$$
A=a=25 \mathrm{~cm} \quad b=c=2 \mathrm{~cm} \quad d=10 \mathrm{~cm} .
$$

The equivalent radius $r=a\left(x+\frac{b^{2}}{24 a^{2}}\right)$

$$
r=25\left(I+\frac{4}{I 5000}\right)=25.00667 \mathrm{~cm} \text {. }
$$

$M$ is now found by using formula $\mathrm{I}, 8$, or $\mathrm{I} 3$, employing $r$ in place of $a$ as the radius.

The result is $M=337.8475$, agreeing very closely with the result found under example 20.

$$
M-M_{0}=\Delta M=.0517 \pi
$$

EXAMPLE 25. LYLE'S FORMULA (28). FOR COILS OF RECTANGULAR SECTION

$$
\begin{gathered}
A=a=25 \quad b=4 \quad c=\mathrm{I} \quad d=\mathrm{IO} \\
r=25\left(\mathrm{I}+\frac{\mathrm{I}}{\mathrm{I} 5000}\right)=25.00167
\end{gathered}
$$

$\beta^{2}=\frac{b^{2}-c^{2}}{\mathrm{I} 2}=\frac{\mathrm{I} 5}{\mathrm{I} 2}=\mathrm{I} .25,2 \beta=2.236 \mathrm{~cm}$, the distance apart of the two filaments which replace the coil (Fig. I4). We now find by formula (I), (8), or (I 3 ) the mutual inductances of two circles I, 2 on the two circles 3,4 , where $a=25.00167$ and $d$ is 7.764 , Io and I $2.236 \mathrm{~cm}$, respectively. Thus :

$$
\begin{aligned}
2 M_{13} & =2 \text { I } 5.00228 \pi \\
M_{14} & =90.3 \mathrm{I} 304 \pi \\
M_{23} & =\text { I } 30.14060 \pi \\
4 M & =435.45592 \pi \\
\therefore M & =\mathrm{I} 08.8640 \pi \\
M_{0} & =\frac{107.4885 \pi}{\mathrm{I} .37 .35 \pi} \\
\Delta M & =
\end{aligned}
$$

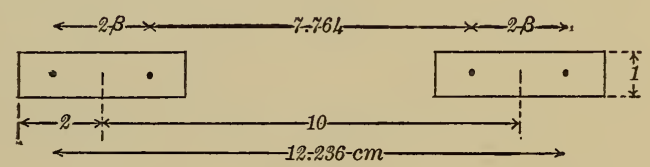

Fig. 14

$\Delta M=$ the correction for section of the coils whose dimensions are given above. These values of $M$ and $\Delta M$ agree nearly with the results obtained in example 22 above. 
EXAMPLE 26. LYLE'S FORMULA (28). FOR UNEQUAL COILS OF RECTANGULAR SECTION

Let us take the same coils as in example 23

$$
\begin{array}{rll}
A=25 & b_{1}=4 & c_{1}=\mathrm{I}
\end{array} \quad d=\mathrm{IO}
$$

For the first coil we find

$$
\begin{aligned}
& r=25\left(I+\frac{c_{1}^{2}}{24 A^{2}}\right)=25.001667 \mathrm{~cm} . \\
& \beta=\text { I.II } 8034 \mathrm{~cm}
\end{aligned}
$$

For the second coil

$$
\begin{aligned}
r & =20\left(\mathrm{I}+\frac{b_{1}^{2}}{24 a^{2}}\right)=20.008333 \mathrm{~cm} \\
\delta & =0.645497 \\
r+\delta & =20.653830 \\
r-\delta & =\mathrm{I} 9.362836
\end{aligned}
$$

We then calculate the mutual inductance of the following pairs of circles:

$\begin{array}{cccc} & A & a & d \\ M_{13} & 25.00 \mathrm{I} 667 & 20.653830 & \mathrm{II} \text { II } 8 \mathrm{O} 34 \\ M_{14} & \text { " } & \mathrm{I} 9.362836 & \mathrm{II} \text { II } 8034 \\ M_{23} & \text { " } & 20.653830 & 8.88 \mathrm{I} 966 \\ M_{24} & \text { " } & \mathrm{I} 9.362836 & 8.88 \mathrm{Ig} 66\end{array}$

The results by Havelock's formula (I6) were

$$
\begin{aligned}
M_{13} & =24 \mathrm{I} .29369 \\
M_{14} & =2 \mathrm{I} 6.9 \mathrm{I} 3 \mathrm{O} 2 \\
M_{23} & =286 . \mathrm{I} 349 \mathrm{O} \\
M_{24} & =255.0347 \mathrm{I} \\
\mathrm{Sum} & =\overline{999.37632} \\
\frac{\mathrm{I}}{4} \mathrm{Sum} & =249.844 \mathrm{I}=\frac{M}{n_{1} n_{2}}
\end{aligned}
$$

which differs from the value by Rayleigh's formula (23) by six or seven in a hundred thousand.

A more accurate value would, in each case, be found if each coil were subdivided and the formulas applied to each of the components as described on page 43 . Such a proceeding is, however, rather tedious, although necessary in precise work. 
EXAMPLE 27. ROSA'S FORMULA (29). FOR COILS OF EQUAL RADII

$$
\begin{aligned}
& A=a=25 \quad b=4 \quad c=\mathrm{I} \quad d=\mathrm{IO} \\
& \text { (same coils as examples 22, 25). } \\
& \log _{e} \frac{8 a}{d}=\log _{e} 20=2.9957 \\
& \frac{3 b^{2}+c^{2}}{96 a^{2}} \cdot \log _{e} \frac{8 a}{d}=\frac{49 \times 2.9957}{60000}=.0024465 \\
& \frac{b^{2}-c^{2}}{\mathrm{I} 2 d^{2}}=\frac{\mathrm{I} 5}{\mathrm{I} 200}=.0125000 \\
& \frac{2 b^{4}+2 c-5 b^{2} c^{2}}{\mathrm{I} 2 \mathrm{O} d^{4}}=\frac{434}{\mathrm{I} 200000}=.00036 \mathrm{I} 7 \\
& \frac{3 b^{6}-3 c^{6}+14 b^{2} c^{4}-14 b^{4} c^{2}}{504 d^{6}}=\frac{8925}{504 \times 10^{6}}=.0000177 \\
& \frac{6 b^{4}+6 c^{4}+5 b^{2} c^{2}}{5760 a^{2} d^{2}}=\frac{1622}{360 \times 10^{6}}=.0000045 \\
& \frac{7 c^{2} d^{2}}{1024 a^{4}}\left(\log _{e} \frac{8 a}{d}-\frac{\mathrm{I} 63}{84}\right) \quad=.0000018 \quad .0153322 \\
& -\frac{\mathrm{II} b^{2}-3 c^{2}}{\mathrm{I} 92 a^{2}}=-\frac{\mathrm{I} 73}{\mathrm{I} 20000}=-.00144 \mathrm{I} 7
\end{aligned}
$$

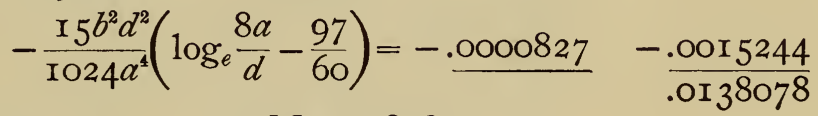

$$
\begin{aligned}
& 4 a=\mathrm{IOO}, \therefore \Delta M=\mathrm{I} .3808 \pi \mathrm{cm} \text {. }
\end{aligned}
$$

This is a little larger value than found by formulas (24) and (28), and we shall see later that it is more nearly correct than either of the other values.

EXAMPLE 28. ROSA'S FORMULAS (30) AND (31). FOR COILS OF EQUAL RADII AND SQUARE SECTION

$$
\begin{aligned}
& A=a=25 \quad b=c=2 \quad d=\mathrm{IO} \\
& \log _{e} \frac{8 a}{d}-\mathrm{I}=2.9957-\mathrm{I}=\mathrm{I} .9957 \\
& \frac{\mathrm{I} 7 b^{2}}{240 d^{2}}=\frac{68}{24000}=\underline{.0028} \quad \mathrm{I.9985} \\
& \frac{-a^{2} b^{2}}{5 d^{4}}=-\frac{2500}{50000}=-.0500 \\
& \frac{-3 d^{2}}{\mathrm{I} 6 a^{2}}\left(\log _{e} \frac{8 a}{d}-\frac{4}{3}\right)=-\frac{300 \times \mathrm{I} .6624}{\mathrm{I} 0000}=-.0499-\frac{.0999}{\mathrm{I} .8986}
\end{aligned}
$$




$$
\frac{b^{2}}{6 a}=\frac{4}{150} \quad \therefore \quad \Delta M=.05063 \pi
$$

The approximate formula (3I) would have given .05I9 (agreeing with formulas 2 I and 27), which would be amply accurate for any experimental purpose. When the section is larger these small terms are, however, more important.

EXAMPLE 29. SECOND EXAMPLE BY FORMULA (30)

$$
\begin{aligned}
& A=a=25 \quad b=c=5 \quad d=10 \\
& \log _{e} \frac{8 a}{d}-\mathrm{I}=\mathrm{I} .9957 \\
& \frac{\mathrm{I} 7 b^{2}}{240 d^{2}}=\underline{.0177} \quad 2.0134 \\
& \frac{-a^{2} b^{2}}{5 d^{4}} \cdot=-.3 \text { I } 25 \\
& \frac{-3 d^{2}}{\mathrm{I} 6 a^{2}}\left(\log _{e} \frac{8 a}{d}-\frac{4}{3}\right)=-. .0499-\frac{.3624}{\mathrm{I} .65 \mathrm{IO}} \\
& \frac{b^{2}}{6 a}=\frac{25}{\mathrm{I}_{50}} \\
& \therefore \Delta M=0.2752 \pi \\
& M_{0}=107.4885 \pi \text { (see example } 20 \text { ) } \\
& \therefore \frac{M}{n_{1} n_{2}}=107.7637 \pi \mathrm{cm} \text {. }
\end{aligned}
$$

This is a very simple formula for computing $\Delta M$, and within a considerable range (i. e., $d$ not larger than $a$ and yet the coils not in contact) it is very accurate.

EXAMPLE 30. FORMULA (32). COILS OF EQUAL RADII, BUT UNEQUAL SECTION

For this we will take the coils of example $2 \mathrm{I}$

$$
\begin{array}{ll}
a=25 & d=\text { IO } \\
b_{1}=4 & b_{2}=2 \\
c_{1}=\mathrm{I} & c_{2}=2
\end{array}
$$




$$
\begin{aligned}
\text { Ist term } & =0.0016227 \\
2 \mathrm{~d} " & =-0.0008542 \\
3 \mathrm{~d} " & =0.0062500 \\
4^{\text {th }} \text { " } & =\frac{0.0000570}{0.0070755} \\
\text { Sum } & =0.70755 \pi \\
\therefore \Delta M & =0.4885 \pi \\
M_{0} & =107.40 .1960 \pi \\
\text { Sum } & =108.9078 \mathrm{~cm} .
\end{aligned}
$$

This example shows that the fourth differentials neglected in (20) here amount to one part in ten thousand.

EXAMPLE 31. ROSA-WEINSTEIN FORMULA (33). FOR COILS OF EQUAL RADII AND EQUAL SECTION

$$
\begin{aligned}
& a=25 \quad b=4 \quad c=\mathrm{I} \quad d=\mathrm{IO} \\
& \alpha_{1}=I_{5} .0000533 \quad \sin ^{2} \gamma=\frac{2500}{2600}=\frac{25}{26} \\
& \alpha_{2}=0.0020267 \quad \cos ^{2} \gamma=\frac{100}{2600}=\frac{I}{26} \\
& \alpha_{3}=0.2170000 \quad \frac{c^{2}}{24 a^{2}}=.0000667 \\
& \alpha_{1}-\alpha_{2}-\alpha_{3}+\left(2 \alpha_{2}-3 \alpha_{3}\right) \cos ^{2} \gamma+8 \alpha_{3} \cos ^{4} \gamma=\mathrm{I} 4.7587 \mathrm{I} 20 \\
& \alpha_{1}+\frac{\alpha_{2}}{2}+2 \alpha_{3}+\left(2 \alpha_{2}+3 \alpha_{3}\right) \cos ^{2} \gamma+8 \alpha_{3} \cos ^{4} \gamma=\mathrm{I}_{5} 4628292 \\
& A=0.0004730 \quad \text { Also } F=3.035 \text { II } 68 \\
& B=0.0123901 \quad E=1.0489686 \\
& (F-E)\left(A+\frac{c^{2}}{24 a^{2}}\right)=0.0010719 \\
& E B=0.0129968 \\
& \mathrm{Sum}=0.0140687 \\
& 4 \pi a \sin \gamma=100 \pi \sqrt{\frac{25}{\mathrm{I} 6}} \therefore \Delta M=\mathrm{I} \cdot 3795 \pi \mathrm{cm} .
\end{aligned}
$$

This is not as simple to calculate as (29) and when $d$ is less than $a / 2$ is less accurate than (29). But for $d=a$ or greater it is more accurate than (29), and indeed the most accurate of all the formulas. 
EXAMPLE 32. FORMULA (34). MUTUAL INDUCTANCE IN TERMS OF SELFINDUCTANCE. FOR COILS RELATIVELY NEAR

For $a=25, b=\mathrm{I}, c=\mathrm{I}$, we have, $n$ being the number of turns in one of the two equal coils,

For $b=2, c=\mathrm{I}$,

$$
L_{1}=4 \pi a n^{2}(4 \cdot \operatorname{I0} 38 \mathrm{I} 6)
$$

For $b=3, c=\mathrm{I}$,

$$
L_{2}=4 \pi a n^{2}(4 \times 3.698695)
$$

$$
L=4 \pi a n^{2}(9 \times 3.4 \operatorname{II} 766)
$$

Then the mutual inductance of I on 3 is by formula (34)

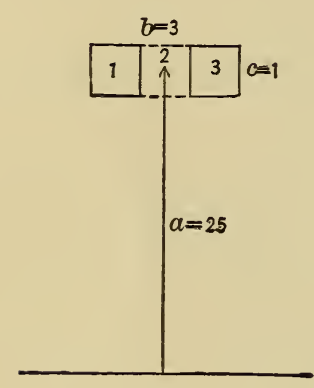

Fig. 15

$$
\begin{aligned}
M & =4 \pi a n^{2}\left[\frac{L+L_{1}-2 L_{2}}{2}\right] \\
& =4 \pi a n^{2}\left[\frac{30.705894+4.103816-29.589560}{2}\right] \\
& =4 \pi a n^{2} \times 2.610075 \\
& =819.979 n^{2} \mathrm{~cm} . \\
\text { If } & n=100,
\end{aligned}
$$

$$
M=8.19979 \text { millihenrys, }
$$

as the mutual inductance of coil I on coil 3 , Fig. I5.

EXAMPLE 33. FORMULA (35). MUTUAL INDUCTANCE BY GEOMETRICAL MEAN DISTANCE

$$
\begin{aligned}
A & =25.1 \\
a & =25.0 \\
b & =c=0.1 \mathrm{~cm} \\
d & =0.1 \mathrm{~cm} .
\end{aligned}
$$

The geometrical mean distance of two coils, corner to corner, as in Fig. IO, is 0.99770I, and $\log \frac{r}{R}=0.002302$

$$
\begin{aligned}
\therefore \Delta M & =100 \times 0.002302(\mathrm{I} .002) \pi \\
& =0.2307 \pi \mathrm{cm} .
\end{aligned}
$$

\section{MUTUAL INDUCTANCE OF COAXIAL SOLENOIDS}

There are several formulas for the calculation of the mutual inductance of coaxial solenoids. Although few of these formulas 
are exact, the approximate formulas often permit inductances to be calculated with very great accuracy by using a sufficient number of terms of the series by which they are expressed.

\section{CONCENTRIC, COAXIAL SOLENOIDS OF EQUAL LENGTH}

MAXWELL'S FORMULA 29

The mutual inductance $M$ of two coaxial solenoids of equal length (Fig. I6) is given by the following expression, due to Maxwell, where $A$ and $a$ are the radii of the outer and inner solenoids, respectively, $l$ is the common length, and $n_{1}$ and $n_{2}$ the number of turns of wire per $\mathrm{cm}$ on the single layer winding of the outer and inner solenoids, respectively:

$$
M=4 \pi^{2} a^{2} n_{1} n_{2}[l-2 A \alpha]
$$

where

$$
\begin{aligned}
& r=\sqrt{l^{2}+A^{2}} \\
& \alpha=\frac{A-r+l}{2 A}-\frac{a^{2}}{\mathrm{I} 6 A^{2}}\left(\mathrm{I}-\frac{A^{3}}{r^{3}}\right)-\frac{a^{4}}{64 A^{4}}\left(\frac{\mathrm{I}}{2}+2 \frac{A^{5}}{r^{5}}-\frac{5}{2} \frac{A^{7}}{r^{7}}\right) \\
& -\frac{35}{2048} \frac{a^{6}}{A^{6}}\left(\frac{1}{7}-\frac{8}{7} \frac{A^{7}}{r^{7}}+4 \frac{A^{9}}{r^{9}}-3 \frac{A^{11}}{r^{11}}\right) \\
& -\frac{53}{2.128} \frac{a^{8}}{A^{8}}\left(\frac{5}{9}+\frac{64}{9} \frac{A^{9}}{r^{9}}-48 \frac{A^{11}}{r^{11}}+88 \frac{A^{13}}{r^{13}}-\frac{\mathrm{I} 43}{3} \frac{A^{15}}{r^{15}}\right) \\
& -\frac{23 \mathrm{I}}{5^{\mathrm{I} 2}{ }^{2}} \frac{a^{10}}{A^{10}}\left(\frac{7}{\mathrm{II}}-\frac{\mathrm{I} 28}{\mathrm{II}} \frac{A^{11}}{r^{11}}+\mathrm{I} 28 \frac{A^{13}}{r^{13}}-4 \mathrm{I} 6 \frac{A^{15}}{r^{15}}+520 \frac{A^{17}}{r^{17}}-22 \mathrm{I} \frac{A^{19}}{r^{19}}\right) \\
& -\frac{429}{2.1024} \frac{a^{12}}{A^{12}}\left(\frac{2 \mathrm{I}}{\mathrm{I} 3}+\frac{5^{\mathrm{I} 2}}{\mathrm{I} 3} \frac{A^{13}}{r^{13}}-640 \frac{A^{15}}{r^{15}}+3200 \frac{A^{17}}{r^{17}}-6800 \frac{A^{19}}{r^{19}}\right. \\
& \left.+6460 \frac{A^{21}}{r^{21}}-226 \mathrm{I} \frac{A^{23}}{r^{23}}\right) \\
& -\frac{6435}{8 \mathrm{I} 92^{2}} \frac{a^{14}}{A^{14}}\left(\frac{\mathrm{II}}{5}-\frac{\mathrm{IO} 24}{\mathrm{I} 5} \frac{A^{15}}{r^{15}}+\mathrm{I} 536 \frac{A^{17}}{r^{17}}-\mathrm{IO} 880 \frac{A^{19}}{r^{19}}+\frac{\mathrm{IO} 3360}{3} \frac{A^{21}}{r^{21}}\right. \\
& \left.-54264 \frac{A^{23}}{r^{23}}+\frac{208012}{5} \frac{A^{25}}{r^{25}}-\frac{37 \text { I } 45}{3} \frac{A^{27}}{r^{27}}\right)-
\end{aligned}
$$

${ }^{29}$ Electricity and Magnetism, Vo1. II, \& 678. 
Putting

$$
M=M_{0}-\Delta M
$$

$M_{0}=4 \pi^{2} a^{2} n_{1} n_{2} l$ is the mutual inductance of an infinite outer solenoid and the finite inner solenoid, while $\Delta M$ is the correction due to the ends.

Equation (36) is Maxwell's expression, except that we have carried it out much further than Maxwell did. We would, however, emphasize that in the great majority of cases only three or four terms need be calculated in $\alpha$, and in these only the first few terms in each parenthesis, to obtain a satisfactory accuracy.

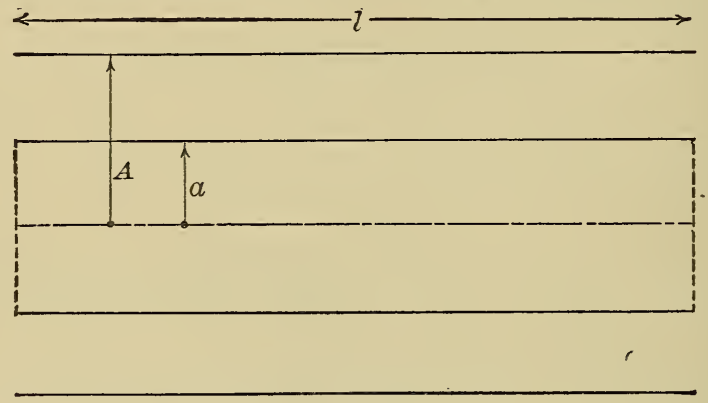

Fig. 16

Since, however, this formula is the most valuable single expression known for the case of solenoids of equal length, it has seemed advisable to extend the series far enough to take care of the most unfavorable cases, which may arise in practice. At the same time the extra terms found have proved of use in checking our extension of Ròiti's formula below.

It should be noticed that the algebraic sums of the coefficients in each of the parentheses is equal to zero. For very long coils $\left(\frac{A}{r}\right.$ small $)$ the quantities in the parentheses are sensibly equal to the absolute term inside. For very short coils the parentheses are a little larger, reaching a maximum in the region $\frac{A}{r}=0.9$, but falling abruptly to zero at the limit $\frac{A}{r}=\mathrm{I}$. The expression for $\alpha$ is 
therefore rapidly convergent for coils of all lengths, even when the inner radius is nearly as great as the outer radius. In such cases the number of terms to be calculated in the above formula may become considerable, but even then it is simpler to use this series than to make the calculation with an absolute formula, such as those of Cohen or Nagaoka.

Equation (36) shows that the mutual inductance is proportional to $l-2 A \alpha$; or the length $l$ must be reduced by $A \alpha$ on each end. When $\alpha$ is small and $l$ is large, $\alpha$ is $I / 2$ approximately. That is, the length $l$ is reduced by $A$, the radius of the outer solenoid.

For the case of two coils each of more than one layer the above formula may be used, $A$ and $a$ being the mean radii, and $n_{1}$ and $n_{2}$ the total number of turns per $\mathrm{cm}$ in all the layers. The result will be only approximate, but usually less in error than if one uses the formula of Maxwell $\S 679$ quoted by Mascart and Joubert. ${ }^{30}$

When the solenoids are very long in comparison with the radii, formula (36) may be simplified by omitting the terms in $A / l, A^{3} / r^{3}$, $A^{5} / \gamma^{5}$, etc. The expression for $\alpha$ then becomes

$$
\alpha=\frac{\mathrm{I}}{2}-\frac{a^{2}}{\mathrm{I} 6 A^{2}}-\frac{a^{4}}{\mathrm{I} 28 A^{4}}-\frac{5 a^{6}}{2048 A^{6}}-\ldots
$$

Heaviside ${ }^{31}$ gives an extension of formula (37), but as it neglects $\frac{A}{l}, \frac{A^{3}}{r^{3}}$, etc., the additional terms are of no importance, being smaller than the terms already neglected in (37).

\section{HAVELOCK'S FORMULA ${ }^{32}$}

This formula for coaxial, concentric solenoids of equal length bears a close resemblance to the preceding, the main difference being that here $l$ enters in place of the quantity $r=\sqrt{l^{2}+A^{2}}$ in

${ }^{30}$ Electricity and Magnetism, Vol. I, p. 533.

31 There are some misprints in Heaviside, 2, p. 277. The radius of the inner solenoid should be $c_{2}$, of the outer $c_{1}$, and $\rho$ is $c_{2}^{2} / c_{1}^{2}$.

32 Phil. Mag., 15, p. 339; I908. There is a misprint in Havelock's equation (25). In the factor outside the brackets, read $a^{2}$ instead of $a$. 
equation (36). Using the same notation as in the latter this formula reads:

$$
M=4 \pi^{2} a^{2} n_{1} n_{2}[l-2 A \beta]
$$

where

$$
\begin{aligned}
\beta=\left[\frac{\mathrm{I}}{2}-\right. & \frac{\mathrm{I}}{\mathrm{I} 6} \frac{a^{2}}{A^{2}}-\frac{\mathrm{I}}{\mathrm{I} 28} \frac{a^{4}}{A^{4}}-\frac{5}{2048} \frac{a^{6}}{A^{6}}-\frac{35}{32768} \frac{a^{8}}{A^{8}}-\cdots \\
& -\frac{\mathrm{I}}{4} \frac{A}{l}+\frac{\mathrm{I}}{\mathrm{I} 6}\left(\mathrm{I}+\frac{a^{2}}{A^{2}}\right)\left(\frac{A}{l}\right)^{3}-\frac{\mathrm{I}}{32}\left(\mathrm{I}+\frac{3 a^{2}}{A^{2}}+\frac{a^{4}}{A^{4}}\right)\left(\frac{A}{l}\right)^{5} \\
& \left.+\frac{5}{25^{6}}\left(\mathrm{I}+6 \frac{a^{2}}{A^{2}}+6 \frac{a^{4}}{A^{4}}+\frac{a^{6}}{A^{6}}\right)\left(\frac{A}{l}\right)^{7}-\cdots \cdot\right]
\end{aligned}
$$

Havelock gives the expressions for the general terms in $\frac{A}{a}$ and $\frac{A}{l}$, so that the computation of $\beta$ may be carried out so as to include terms of higher order when necessary. These expressions are

$$
-\frac{(2 n-\mathrm{I})[\mathrm{I} \cdot 3 \cdot 5 \cdots \cdot(2 n-3)]^{2}}{2^{2 n+1} n !(n+\mathrm{I}) !}\left(\frac{a}{A}\right)^{2 n}
$$

and

$$
\frac{(-\mathrm{I})^{s}(2 s) ! F\left(-s-\mathrm{I},-s, 2, \frac{a^{2}}{A^{2}}\right)}{2^{2 s+2} s !(s+\mathrm{I}) !}\left(\frac{A}{l}\right)^{2 s+1}
$$

where $F$ is.a hypergeometric series in $\frac{a^{2}}{A^{2}}$, all of whose terms after that in $\left(\frac{a}{A}\right)^{2 s}$ are zero.

$$
\begin{aligned}
F(\alpha, \beta, \gamma, z)=\mathrm{I}+\frac{\alpha \beta}{\mathrm{I} \cdot \gamma} z & +\frac{\alpha(\alpha+\mathrm{I}) \beta(\beta+\mathrm{I})}{\mathrm{I} \cdot 2 \cdot \gamma(\gamma+\mathrm{I})} z^{2} \\
& +\frac{\alpha(\alpha+\mathrm{I})(\alpha+2) \beta(\beta+\mathrm{I})(\beta+2)}{\mathrm{I} \cdot 2 \cdot 3 \cdot \gamma(\gamma+\mathrm{I})(\gamma+2)} z^{3}+\cdots
\end{aligned}
$$

Formula (38) may be regarded as intermediate between $\left(j^{6}\right)$ and (37), being applicable only to coils whose length is greater than the radius of the larger coil. In such cases, however, it furnishes a valuable check on Maxwell's formula. 
CONCENTRIC COAXIAL SOLENOIDS, INNER COIL SHORTER THAN THE OUTER

\section{RÒITI'S FORMULA}

For a pair of concentric, coaxial solenoids of which the inner solenoid is shorter than the outer, we have the following: ${ }^{33}$

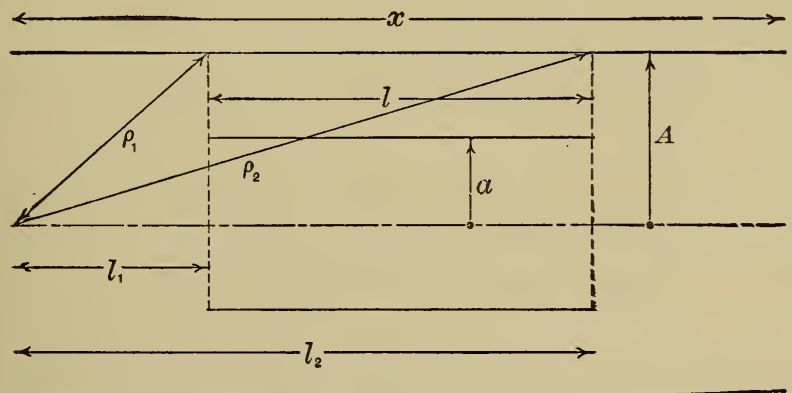

Fig. 17

$$
\begin{aligned}
& M=4 \pi^{2} a^{2} n_{1} n_{2}\left[\rho_{2}-\rho_{1}+\frac{a^{2} A^{2}}{8}\left(\frac{\mathrm{I}}{\rho_{1}^{3}}-\frac{\mathrm{I}}{\rho_{2}^{3}}\right)-\frac{a^{4} A^{2}}{\mathrm{I} 6}\left(\frac{\mathrm{I}}{\rho_{1}^{5}}-\frac{\mathrm{I}}{\rho_{2}^{5}}\right)\right. \\
&+\frac{5}{64} a^{4} A^{4}\left(\mathrm{I}+\frac{\mathrm{I}}{2} \frac{a^{2}}{A^{2}}\right)\left(\frac{\mathrm{I}}{\rho_{1}^{7}}-\frac{\mathrm{I}}{\rho_{2}^{7}}\right) \\
& \quad-\frac{35}{256} a^{6} A^{4}\left(\mathrm{I}+\frac{\mathrm{I}}{5} \frac{a^{2}}{A^{2}}\right)\left(\frac{\mathrm{I}}{\rho_{1}^{9}}-\frac{\mathrm{I}}{\rho_{2}^{9}}\right) \\
&+\frac{\mathrm{IO} 5}{\mathrm{IO} 24} a^{6} A^{6}\left(\mathrm{I}+\frac{9}{5} \frac{a^{2}}{A^{2}}+\frac{\mathrm{I}}{5} \frac{a^{4}}{A^{4}}\right)\left(\frac{\mathrm{I}}{\rho_{1}^{11}}-\frac{\mathrm{I}}{\rho_{2}^{11}}\right) \\
&-\frac{693}{2048} a^{8} A^{6}\left(\mathrm{I}+\frac{2}{3} \frac{a^{2}}{A^{2}}+\frac{\mathrm{I}}{2 \mathrm{I}} \frac{a^{4}}{A^{4}}\right)\left(\frac{\mathrm{I}}{\rho_{1}^{13}}-\frac{\mathrm{I}}{\rho_{2}^{13}}\right) \\
&+\frac{3003}{\mathrm{I} 6384} a^{8} A^{8}\left(\mathrm{I}+4 \frac{a^{2}}{A^{2}}+\frac{\mathrm{IO}}{7} \frac{a^{4}}{A^{4}}+\frac{\mathrm{I}}{\mathrm{I} 4} \frac{a^{6}}{A^{6}}\right)\left(\frac{\mathrm{I}}{\rho_{1}^{15}}-\frac{\mathrm{I}}{\rho_{2}^{15}}\right)-. .
\end{aligned}
$$

in which (see Fig. I7)

${ }^{33}$ For the derivation and method of extension of this formula see this Bulletin, $\mathbf{3}$, pp. 309-310. Recently we have carried it out still further to include the case of coils of moderate length. This formula was originally given (without proof and including the main term in $\left(\frac{\mathrm{I}}{\rho_{i}^{7}}-\frac{\mathrm{I}}{\rho_{2}^{7}}\right)$ only) in this Bulletin, 2, p. I30; I906. 


$$
\begin{aligned}
& \rho_{1}=\sqrt{l_{1}^{2}+A^{2}} \text { where } l_{1}=\frac{x-l}{2} \\
& \rho_{2}=\sqrt{l_{2}^{2}+A^{2}} \quad \text { " } \quad l_{2}=\frac{x+l}{2}
\end{aligned}
$$

$l=l_{2}-l_{1}=$ length of inner solenoid.

$x=$ length of outer solenoid and $A$ and $a$ the radii.

When $\frac{l}{x}$ is small (case of short inner coil), $\left(\rho_{2}-\rho_{1}\right)$ is most accurately calculated by the exact formula $\left(\rho_{2}-\rho_{1}\right)=\frac{x l}{\rho_{1}+\rho_{2}}$, the denominator being calculated from the above expressions for $\rho_{1}$ and $\rho_{2}$.

For long coils $\left(\frac{2 A}{x}\right.$ small $)$ the above formula is rapidly convergent, especially if the inner coil is considerably shorter than the outer. This formula may also be used for short coils $\left(\frac{x}{2 A}\right.$ small $)$, the convergence being most rapid when the radius of the inner coil is small in comparison with that of the outer. For very short coils, we have expanded formula (39) in a series in ascending powers of $\frac{a^{2}}{A^{2}}$. This formula is, however, not so accurate, nor so simple to use as that of Searle and Airey, and has not been included in this collection.

A peculiarity of Ròiti's formula is that the successive terms, especially in the case of short coils, are nearly equal in pairs. Thus the terms in $\left(\frac{I}{\rho_{1}^{5}}-\frac{I}{\rho_{2}^{5}}\right)$ and $\left(\frac{I}{\rho_{1}^{7}}-\frac{I}{\rho_{2}^{7}}\right)$ are of the same order of magnitude, but of opposite sign; similarly for the terms involving the ninth and eleventh powers of $\rho_{1}$ and $\rho_{2}$, and so on. For the limiting case $x=l$, Ròiti's formula goes over into Maxwell's (36), as would be expected, since both are derived by integration of the same original expression between appropriate limits. To obtain, however, the same precision, twice as many terms have to be calculated in Ròiti's formula as in Maxwell's. We see from these considerations, that in using Ròiti's formula, the inner coil need not be very different in length from the outer coil, although in general the convergence is better with a relatively short inner solenoid. 


\section{GRAY'S FORMULA}

Gray $^{34}$ gives a general expression for the mutual kinetic energy of two solenoidal coils which may or may not be concentric, and their axes may be at any angle $\phi$. The most important case in practice is when the two coils are coaxial. In that case the zonal harmonic factors in each term reduce to unity, and half the terms become zero. Putting the current in each equal to unity, the mutual kinetic energy becomes the mutual inductance $M$.

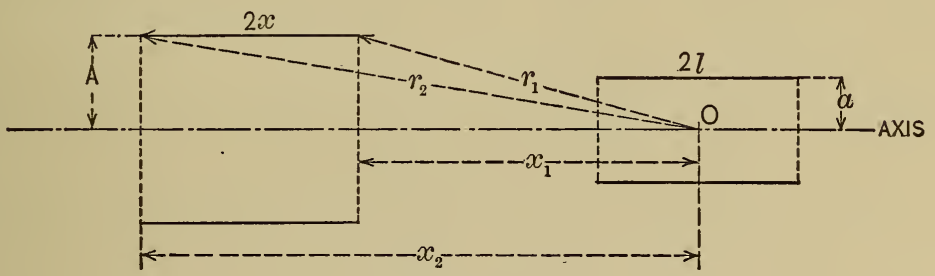

Fig. 18

Let $2 x, A, n_{1}$ be respectively the length, radius, and number of turns per $\mathrm{cm}$ of one of the coils, and $2 l, a, n_{2}$ be the corresponding quantities for the other solenoid. Let, further, $x_{1}$ and $x_{2}$ be the distances, along the axis, between the center of the coil with radius $a$ and the nearer and further end planes, respectively, of the coil with radius $A$, and let $r_{1}$ and $r_{2}$ be the diagonals (Fig. I8).

$$
r_{1}=\sqrt{x_{1}^{2}+A^{2}} \quad r_{2}=\sqrt{x_{2}^{2}+A^{2}}
$$

Gray's expression with these changes becomes

$$
M=\pi^{2} a^{2} A^{2} n_{1} n_{2}\left[K_{1} k_{1}+K_{3} k_{3}+K_{5} k_{5}+\cdots \cdot\right]
$$

where $K_{1}, K_{3}$, etc., are functions of $x$ and $A$, and $k_{1}, k_{3}$, etc., are functions of $l$ and $a .^{35}$

${ }^{34}$ Absolute Measurements, 2, Part I, p. 274, equation 53.

${ }^{35}$ Rosa, this Bulletin, 3, p. 22I; I907. 


$$
\begin{aligned}
& K_{1}=\frac{2}{A^{2}}\left(\frac{x_{2}}{r_{2}}-\frac{x_{1}}{r_{1}}\right) \\
& K_{3}=\frac{\mathrm{I}}{2}\left(\frac{x_{1}}{r_{1}^{5}}-\frac{x_{2}}{r_{2}^{5}}\right) \\
& K_{5}=-\frac{A^{2}}{8}\left\{\frac{x_{1}}{r_{1}^{9}}\left(3-4 \frac{x_{1}^{2}}{A^{2}}\right)-\frac{x_{2}}{r_{2}^{9}}\left(3-4 \frac{x_{2}^{2}}{A^{2}}\right)\right\} \\
& =-\frac{A^{2}}{8}\left\{\frac{x_{1}}{r_{1}^{9}} X_{2}^{\prime}-\frac{x_{2}}{r_{2}^{9}} X_{2}^{\prime \prime}\right\} \\
& K_{7}=\frac{A^{4}}{8}\left\{\frac{x_{1}}{r_{1}^{13}}\left(\frac{5}{2}-\text { Io } \frac{x_{1}^{2}}{A^{2}}+4 \frac{x_{1}^{4}}{A^{4}}\right)-\frac{x_{2}}{r_{2}^{13}}\left(\frac{5}{2}-\text { Io } \frac{x_{2}^{2}}{A^{2}}+4 \frac{x_{2}^{4}}{A^{4}}\right)\right\} \\
& =\frac{A^{4}}{8}\left\{\frac{x_{1}}{r_{1}^{13}} X_{4}^{\prime}-\frac{x_{2}}{r_{2}^{13}} X_{4}^{\prime \prime}\right\} \\
& k_{1}=2 l \\
& k_{3}=-a^{2} l\left(3-4 \frac{l^{2}}{a^{2}}\right)=-a^{2} l L_{2} \\
& k_{5}=a^{4}<\left(\frac{5}{2}-10 \frac{l^{2}}{a^{2}}+4 \frac{l^{4}}{a^{4}}\right)=a^{4} l L_{4} \\
& k_{7}=-a^{6} l\left(\frac{35}{\mathrm{I} 6}-\frac{35}{2} \frac{l^{2}}{a^{2}}+2 \mathrm{I} \frac{l^{4}}{a^{4}}-4 \frac{l^{6}}{a^{6}}\right)=-a^{6} l L_{6}
\end{aligned}
$$

This formula is simple and convenient for calculation, if only a few terms need be evaluated. This is the case when $r_{1}$ and $r_{2}$ are large (coils relatively far apart). The coefficients $L_{2 n}, X^{\prime}{ }_{2 n}$ and $X^{\prime \prime}{ }_{2 n}$ are derived from the same polynomial $S_{2 n}$ by substituting $\frac{l^{2}}{a^{2}} \frac{x_{1}^{2}}{A^{2}}$, and $\frac{x_{2}^{2}}{A^{2}}$, respectively.

For short coils relatively far apart these polynomials are all small. Table XVIII gives values of the polynomial $S_{2 n}$ with varying argument, to aid in calculations where great accuracy is not desired, or to aid in making preliminary calculations to see whether the convergence will be satisfactory in any particular case.

If the coils are concentric, and the ratio of the length of the winding of the outer coil to the radius is $\sqrt{3}$ to $\mathrm{I}, K_{5}=\mathrm{O}$, and if the same condition holds for the inner coil, $k_{3}=0$. If in addition $a$ is considerably smaller than $A$, the terins of higher order become negligible and (40) reduces to

$$
M=\frac{2 \pi^{2} a^{2} N_{1} N_{2}}{d}
$$


where $d$ is half the diagonal of the outer coil, $=\sqrt{x^{2}+A^{2}}$. When the dimensions depart slightly from these theoretical ratios the small correction terms to (4I) can be calculated..$^{35 a}$ The general case for concentric coils is treated in the next section.

\section{SEARLE AND AIREY'S FORMULA}

The following expression for the mutual inductance of two concentric, coaxial solenoidal coils (Fig. I9) has been given by Searle and Airey : ${ }^{36}$

$$
\begin{aligned}
M & =g_{1} G_{1}+g_{3} G_{3}+g_{5} G_{5}+g_{7} G_{7}+\ldots . \\
& =\frac{2 \pi^{2} a^{2} N_{1} N_{2}}{d}\left[\mathrm{I}-\frac{A^{2}}{2 d^{4}} \frac{4 l^{2}-3 a^{2}}{4}-\frac{A^{2}\left(4 x^{2}-3 A^{2}\right)}{8 d^{8}} \cdot \frac{8 l^{4}-20 l^{2} a^{2}+5 a^{4}}{8}\right. \\
& \left.-\frac{A^{2}\left(8 x^{4}-20 x^{2} A^{2}+5 A^{4}\right)}{\mathrm{I} 6 d^{12}} \cdot \frac{\left(64 l^{6}-336 l^{4} a^{2}+280 l^{2} a^{4}-35 a^{6}\right)}{64}-\ldots\right]
\end{aligned}
$$

The notation of (42) differs slightly from that used by Searle and Airey.

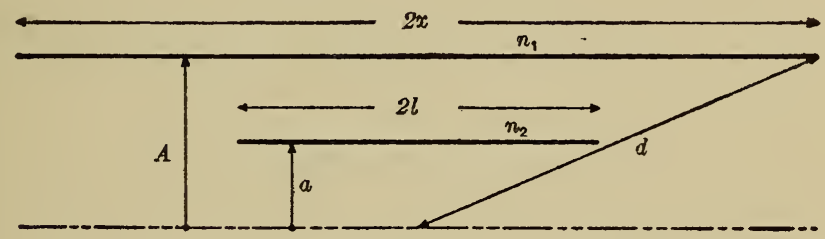

Fig. 19

Equation (42) has been extended and put for greater convenience in calculation into the form ${ }^{37}$ shown on next page.

${ }^{35} a$ Rosa, this Bulletin, 3, p. 22I; 1907.

${ }^{36}$ The Electrician (London), 56, p. 318 ; 1905.

${ }^{37}$ Rosa, this Bulletin, 3, p. 224; 1907.

$2 \mathrm{I} 674^{\circ}-\mathrm{I} 2-5$ 
$M=\frac{2 \pi^{2} a^{2} N_{1} N_{2}}{d}\left[\mathrm{I}+\frac{A^{2} a^{2}}{8 d^{4}} L_{2}+\frac{A^{4} a^{4}}{32 d^{8}} X_{2} L_{4}\right.$

$$
\begin{aligned}
& +\frac{A^{6} a^{6}}{32 d^{12}} X_{4} L_{6}+\frac{A^{8} a^{8}}{32 d^{16}} X_{6} L_{8}+\ldots . \\
& \left.+\frac{\mathrm{I}}{32}\left(\frac{A a}{d^{2}}\right)^{2 n} X_{2 n-2} L_{2 n}+\ldots . .\right]
\end{aligned}
$$

where

$$
\begin{array}{rlrl}
X_{2} & =3-4 \frac{x^{2}}{A^{2}} & L_{2} & =3-4 \frac{l^{2}}{a^{2}} \quad d=\sqrt{x^{2}+A^{2}} \\
X_{4} & =\frac{5}{2}-\mathrm{IO} \frac{x^{2}}{A^{2}}+4 \frac{x^{4}}{A^{4}} & L_{4} & =\frac{5}{2}-\mathrm{IO} \frac{l^{2}}{a^{2}}+4 \frac{l^{4}}{a^{4}} \\
X_{6} & =\frac{35}{\mathrm{I} 6}-\frac{35}{2} \frac{x^{2}}{A^{2}}+2 \mathrm{I} \frac{x^{4}}{A^{4}}-4 \frac{x^{6}}{A^{6}} & L_{6} & =\frac{35}{\mathrm{I} 6}-\frac{35}{2} \frac{l^{2}}{a^{2}}+2 \mathrm{II} \frac{l^{4}}{a^{4}}-4 \frac{l^{6}}{a^{6}} \\
X_{8} & =\frac{63}{32}-\frac{\text { IO } 5}{4} \frac{x^{2}}{A^{2}}+63 \frac{x^{4}}{A^{4}} & L_{8} & =\frac{63}{32}-\frac{\text { IO } 5}{4} \frac{l^{2}}{a^{2}}+63 \frac{l^{4}}{a^{4}}-36 \frac{l^{6}}{a^{6}}+4 \frac{l^{8}}{a^{8}} \\
- & 36 \frac{x^{6}}{A^{6}}+4 \frac{x^{8}}{A^{8}} & L_{10} & =\frac{23 \mathrm{I}}{\mathrm{I} 28}-\frac{\mathrm{II} 55}{32} \frac{l^{2}}{a^{2}}+\frac{\text { II } 55}{8} \frac{l^{4}}{a^{4}}-\mathrm{I} 65 \frac{l^{6}}{a^{6}} \\
& +55 \frac{l^{8}}{a^{8}}-4 \frac{l^{10}}{a^{10}}
\end{array}
$$

$N_{1}=2 x n_{1}$ and $N_{2}=2 \ln _{2}$ are the total number of turns on the two solenoids. This formula reduces to (4I) when the terms after the first are negligible, as they are when the conditions assumed for (4I) are fulfilled. The above expressions for $L_{2}, X_{2}$ show what these conditions are in order to make the second and third terms zero. If $l^{2} / a^{2}$ is slightly more or less than $3 / 4,(43)$ gives the value of the second term which is neglected in (4I), etc.

The degree of convergence of Searle and Airey's formula depends primarily on the magnitude of the quantity $\frac{A^{2} a^{2}}{d^{4}}$; in certain cases, however, the values of the coefficients become of equal importance, making it necessary to examine carefully into the degree of convergence of the formula, since the terms of higher order are sometimes larger than those immediately preceding. Since the $X$ and $L$ 
coefficients are polynomials in $\frac{l^{2}}{a^{2}}$ and $\frac{x^{2}}{A^{2}}$, each one will have a finite number of roots depending on the degree of the polynomial. The values of these coefficients will therefore, with increasing $\frac{l}{a}$ or $\frac{x}{A}$, oscillate between positive and negative values, each maximum or minimum being greater than that preceding, until, for values of the argument greater than the largest root, the values of the functions increase indefinitely without limit.

For short coils $\left(\frac{x}{A}\right.$ and $\frac{l}{a}$ small $)$ the coefficients will evidently be confined to moderate values, and if, further, the inner radius is small relatively to the outer, the convergence will be very rapid. For longer coils the coefficients may attain very large values, and the convergence become very unsatisfactory, in spite of the fact that $\frac{A^{2} a^{2}}{d^{4}}$ is, for given radii, smaller with long coils than with short coils. The conditions are so complicated that we have calculated (Table XIX) certain values of the coefficients to aid in deciding whether, in any given case, the convergence will be satisfactory or not. The values given for $\frac{x}{A}$ and $\frac{l}{a}$ less than unity will also be found useful in calculations of the mutual inductance of short coils by Searle and Airey's formula, when the highest precision is not required. Coefficients of higher order than those given above are calculated by the formula

$L_{2 n}=\sum_{p=0}^{p=n} \frac{(-\mathrm{I})^{n-p}(2 n+\mathrm{I}) 2 n(2 n-\mathrm{I}) \cdots \cdot[2 n-(2 p-2)]}{\left(\frac{p+\mathrm{I}}{4}\right) 2^{2} \cdot 4^{2} \cdot 6^{2} \cdots \cdots(2 p)^{2}}\left(\frac{l}{a}\right)^{2 n-2 p}$

$X_{2 n}$ is calculated by the same expression in $\frac{x}{A}$ instead of $\frac{l}{a}$.

Table XVIII includes all the positive and negative maxima as well as the zero points of the coefficients up to and including $L_{14}$ or $X_{14}$, together with the values at a number of intermediate points. Although, from the nature of the case, a table to serve as the basis of accurate calculations would be somewhat bulky, those given should suffice to simplify the use of this valuable formula. 
COHEN'S FORMULA ${ }^{38}$ FOR ANY TWO COAXIAL, CONCENTRIC SOLENOIDS

This is an absolute formula for two coaxial, concentric solenoids of lengths $2 l_{1}$ and $2 l_{2}$, Fig. 20 .

$$
\begin{aligned}
M=4 \pi n_{1} n_{2}\left(V-V_{1}\right) \\
V=-\left(A^{2}-a^{2}\right) c\left[F\left\{F\left(k^{\prime}, \theta\right)-E\left(k^{\prime}, \theta\right)\right\}-E F\left(k^{\prime}, \theta\right)\right] \\
+\frac{c^{4}-\left(A^{2}-6 A a+a^{2}\right) c^{2}-2\left(A^{2}-a^{2}\right)^{2}}{3 \sqrt{(A+a)^{2}+c^{2}}} \cdot F \\
+\frac{2\left(A^{2}+a^{2}\right)-c^{2}}{3} \sqrt{(A+a)^{2}+c^{2}} \cdot E-c\left(A^{2}-a^{2}\right) \frac{\pi}{2}
\end{aligned}
$$

$V_{1}$ is obtained from $V$ by replacing $c$ by $c_{1}$,

$$
c=l_{1}+l_{2} \quad c_{1}=l_{1}-l_{2},
$$

$F$ and $E$ are the complete elliptic integrals of the first and second kind to modulus $k$, where $k^{2}=\frac{4 A a}{(A+a)^{2}+c^{2}}$

$F\left(k^{\prime}, \theta\right)$ and $E\left(k^{\prime}, \theta\right)$ are the incomplete elliptic integrals of modulus $k^{\prime}$ and amplitude $\theta$,

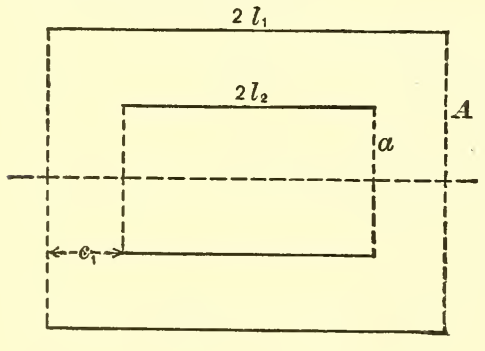

$$
\begin{aligned}
k^{\prime 2} & =\mathrm{I}-k^{2}=\mathrm{I}-\frac{4 A a}{(A+a)^{2}+c^{2}} \\
& =\frac{(A-a)^{2}+c^{2}}{(A+a)^{2}+c^{2}} \\
\sin ^{2} \theta & =\frac{\left(A^{2}-a^{2}\right)^{2}+c^{2}(A-a)^{2}}{\left(A^{2}-a^{2}\right)^{2}+c^{2}(A+a)^{2}}
\end{aligned}
$$

Fig. 20

\section{NAGAOKA'S FORMULA FOR ANY COAXIAL SOLENOIDS}

Nagaoka has recently given ${ }^{39}$ an absolute formula for the mutual inductance of two coaxial solenoids, whether concentric or not, and

${ }^{38}$ This Bulletin, 3, p. 30r; I907.

${ }^{39}$ Jour. Coll. Sci., Tokyo, 27, art. 6; 1909. There are a number of misprints in Nagaoka's article, which we have detected and corrected by a careful check on the derivation of the formulas. 
has expanded this in $q$ functions in a form suitable for calculation. In the notation of Fig. I8,

$$
M=4 \pi n_{1} n_{2} A a\left(I_{1}-I_{2}-I_{3}+I_{4}\right)
$$

where $I_{1}, I_{2}, I_{3}$, and $I_{4}$ are the values of the integral $I$, given below with the arguments

$$
c=d+(x+l), d+(x-l), d-(x-l) \text { and } d-(x+l)
$$

respectively, where $d$ is the distance between centers.

The expression for $I$ is, in the Weierstrassian notation,

$$
I=2\left\{\left(\frac{g_{2}}{6}-\mathrm{p}^{2} v\right) \omega_{1}+\mathrm{p} v \cdot \eta_{1}+\frac{\mathbf{p}^{\prime} v}{2}\left(\eta_{1} v-\omega_{1} \frac{\sigma^{\prime}}{\sigma}(v)\right)\right\}
$$

where $v$ is an auxiliary quantity, and $\omega_{1}$ and $g_{2}$ are respectively the real semiperiod and invariant of the Weierstrassian function $\mathrm{p} u$.

To calculate $I$, Nagaoka divides it in two parts

$$
\begin{aligned}
& I^{\prime}=\left(\frac{g_{2}}{6}-\mathrm{p}^{2} v\right) \omega_{1}+\mathrm{p} v \cdot \eta_{1} \\
& I^{\prime \prime}=\frac{\mathrm{p}^{\prime} v}{2}\left(\eta_{1} v-\omega_{1} \frac{\sigma^{\prime}}{\sigma}(v)\right)
\end{aligned}
$$

We then calculate the following auxiliary quantities

$$
\begin{aligned}
& \alpha=\left(\frac{2}{A \alpha}\right)^{\frac{1}{3}} \\
& P_{1}=\left(\mathrm{p} v-e_{1}\right)=-\frac{c^{3}}{2 A a \alpha} \\
& P_{2}=\left(\mathrm{p} v-e_{2}\right)=\frac{(A-a)^{3}}{2 A a \alpha} \\
& P_{3}=\left(\mathrm{p} v-e_{3}\right)=\frac{(A+a)^{2}}{2 A a \alpha}
\end{aligned}
$$

and thence $\left(e_{1}-e_{2}\right),\left(e_{1}-e_{3}\right)$, and $\left(e_{2}-e_{3}\right)$, which with the relation $\left(e_{1}+e_{2}+e_{3}\right)=0$ enable us to find $\mathrm{p} v$.

The very small quantity $q$ is found, as in formula (8), by the relations (see also Table $\mathrm{XV}$ )

$$
\begin{gathered}
q=\frac{l}{2}+2\left(\frac{l}{2}\right)^{5}+\mathrm{I} 5\left(\frac{l}{2}\right)^{9}+\cdots \\
k^{2}=\frac{e_{2}-e_{3}}{e_{1}-e_{3}} \quad k^{\prime 2}=\frac{e_{1}-e_{2}}{e_{1}-e_{3}} \quad l=\frac{\mathrm{I}-\sqrt{k^{\prime}}}{\mathrm{I}+\sqrt{k^{\prime}}}=\frac{k^{2}}{\left(\mathrm{I}+k^{\prime}\right)\left(\mathrm{I}+\sqrt{k^{\prime}}\right)^{2}}
\end{gathered}
$$


and $\omega_{1}$ may be calculated by any one of the following equations (the other two serving as checks):

$$
\begin{aligned}
& \omega_{1}=\frac{2 \pi \sqrt{q}}{\sqrt{e_{2}-e_{3}}}\left(\mathrm{I}+q^{2}+q^{6}+q^{12}+\cdots \cdot\right)^{2} \\
& \omega_{1}=\frac{\pi}{2 \sqrt{e_{1}-e_{3}}}\left(\mathrm{I}+2 q+2 q^{4}+2 q^{9}+\cdots \cdot\right)^{2} \\
& \omega_{1}=\frac{\pi}{2 \sqrt{e_{1}-e_{2}}}\left(\mathrm{I}-2 q+2 q^{4}-2 q^{9}+\cdots \cdot\right)^{2}
\end{aligned}
$$

The term $I^{\prime}$ is now given by either of the following two formulas, which give a check on the calculation:

$$
\begin{aligned}
& I^{\prime}=-\left\{\frac{P_{1}\left(P_{2}+P_{3}\right)}{2}+\frac{\left(P_{1}+2 P_{2}\right)\left(e_{2}-e_{3}\right)}{6}\right\} \omega_{1}-\frac{\mathrm{p} v}{4 \omega_{1}} \frac{\theta_{1}^{\prime \prime}{ }_{3}(\mathrm{o})}{\theta_{3}(\mathrm{o})} \\
& I^{\prime}=-\left\{\frac{P_{1}\left(P_{2}+P_{3}\right)}{2}-\frac{\left(P_{1}+2 P_{3}\right)\left(e_{2}-e_{3}\right)}{6}\right\} \omega_{1}-\frac{\mathrm{p} v}{4 \omega_{1}} \frac{\theta_{0}{ }^{\prime \prime}(\mathrm{o})}{\theta_{0}(\mathrm{o})}
\end{aligned}
$$

The quotients of the $\theta$ functions are easily calculated from the known value of $q$ and the relations

$$
\begin{aligned}
& \frac{\theta_{3}^{\prime \prime}(\mathrm{o})}{\theta_{3}(\mathrm{o})}=-\frac{8 \pi^{2}\left(q+4 q^{4}+9 q^{9}+\cdots \cdot\right)}{\mathrm{I}+2 q+2 q^{4}+2 q^{9}+\cdots \cdot} \\
& \frac{\theta_{0}^{\prime \prime}(\mathrm{o})}{\theta_{0}(\mathrm{o})}=\frac{8 \pi^{2}\left(q-4 q^{4}+9 q^{9}-\cdots \cdot \cdot\right)}{\mathrm{I}-2 q+2 q^{4}-2 q^{9}+\cdots \cdot \cdot}
\end{aligned}
$$

To calculate $I^{\prime \prime}$ we have

$$
I^{\prime \prime}=-\frac{\pi c}{8 A a}\left(A^{2}-a^{2}\right)\left[\sqrt{\frac{b-\mathrm{I}}{b+\mathrm{I}}}+4 q^{2} \sqrt{b^{2}-\mathrm{I}}\left\{\mathrm{I}-q^{2}(2 b-\mathrm{r})\right\}\right]
$$

the expression in the brackets being nearly equal to unity. The quantity $b$ is calculated from the equations

$$
\begin{aligned}
-b & =\cos 2 \pi w=\frac{s}{q}\left(\mathrm{I}+2 q^{4} \cos 4 \pi w+\cdots\right) \\
s & =\frac{\mathrm{I} \sqrt[4]{e_{1}-e_{3}} \sqrt{P_{2}}-\sqrt[4]{e_{1}-e_{2}} \sqrt{P_{3}}}{2 \sqrt[4]{e_{1}-e_{3}} \sqrt{P_{2}}+\sqrt[4]{e_{1}-e_{2}} \sqrt{P_{3}}} .
\end{aligned}
$$


first putting $\cos 2 \pi w$ equal to its approximate value $\frac{s}{q}$, and then computing the small correction in $\cos 4 \pi w$ from $\cos 2 \pi w$, remembering that $w$ is a pure imaginary. The correction to $b$ thus found is often negligible.

The term $I^{\prime \prime}$ becomes less important as the difference of the radii of the solenoids becomes small, and vanishes for equal radii. If, further, the lengths of the solenoids be equal also, $I_{2}=I_{3}$, and we have only three of the integrals to evaluate, and only the first term $I^{\prime}$ in each of these.

For concentric, coaxial solenoids $d=0$, and consequently $I_{1}-I_{2}=I_{1}-I_{3}$, so that only two integrals must be calculated.

On account of the number of auxiliary quantities involved, Nagaoka's formula should not be employed except when the various series formulas given in this section are all shown to be inadequate. It is, however, simpler to use Nagaoka's formula than the elliptic integral formula from which it is derived, or any other expression in incomplete integrals yet derived, even supposing Legendre's table of incomplete integrals to be available.

\section{RUSSELL'S FORMULAS ${ }^{40}$}

Russell's formula for coaxial solenoids in the notation of this paper is

$$
\begin{aligned}
M=4 \pi^{2} a^{2} n_{1} n_{2}\left[R _ { 1 } \left\{\mathrm{I}-\frac{\mathrm{I}}{2} q_{2} k_{1}{ }^{2}-\frac{\mathrm{I}}{2} \cdot \frac{\mathrm{I}}{4} q_{3} k_{1}{ }^{4}-\frac{\mathrm{I} \cdot \mathrm{I} \cdot 3}{2 \cdot 4 \cdot 6} q_{4} k_{1}{ }^{6}\right.\right. & \\
& \left.-\frac{\mathrm{I} \cdot \mathrm{I} \cdot 3 \cdot 5}{2 \cdot 4 \cdot 6 \cdot 8} q_{5} k_{1}{ }^{8}-\frac{\mathrm{I} \cdot \mathrm{I} \cdot 3 \cdot 5 \cdot 7}{2 \cdot 4 \cdot 6 \cdot 8 \cdot \mathrm{IO}} q_{6} k_{1}{ }^{10}-\cdots \cdot\right\} \\
& \left.-R_{2}\left\{\mathrm{I}-\frac{\mathrm{I}}{2} q_{2} k_{2}{ }^{2}-\frac{\mathrm{I}}{2} \cdot \frac{\mathrm{I}}{4} q_{3} k_{2}{ }^{4}-\text { terms with above coefs. }\right\}\right][46]
\end{aligned}
$$

where

$$
\begin{array}{ll}
R_{1}{ }^{2}=(A+a)^{2}+\left(l_{1}+l_{2}\right)^{2} & k_{1}{ }^{2}=\frac{4 A a}{R_{1}{ }^{2}} \\
R_{2}{ }^{2}=(A+a)^{2}+\left(l_{1}-l_{2}\right)^{2} & k_{2}{ }^{2}=\frac{4 A a}{R_{2}{ }^{2}}
\end{array}
$$




$$
\begin{aligned}
& q_{n}=\frac{(A+a)^{2}}{4 A a} q_{n-1}-\frac{\mathrm{I} \cdot 3 \cdot 5 \ldots 2 n-3}{n 24 \cdot 6 \ldots 2 n-2} \frac{A}{a} \\
& q_{2}=\frac{(A+a)^{2}}{4 A a}-\frac{\mathrm{I} \mathrm{I}}{22} \frac{A}{a} \\
& q_{3}=\frac{(A+a)^{2}}{4 A a} q_{2}-\frac{\mathrm{I} \mathrm{I} \cdot 3 \frac{A}{32 \cdot 4}}{a}
\end{aligned}
$$

etc.

$A$ and $a$ are the radii of the outer and inner cylinders respectively, $2 l_{1}$ and $2 l_{2}$ their lengths, Fig. 20, and $n_{1}, n_{2}$ the number of turns of wire per $\mathrm{cm}$ in the two windings. This formula applies only when the inner coil is shorter than the outer. For two coils of equal length the second part of the above formula is not convergent, and hence it must be replaced by an expression in elliptic integrals. The formula thus becomes (equation 42 in Russell's paper)

$$
\begin{gathered}
M=4 \pi^{2} a^{2} n_{1} n_{2}\left[R_{1}\left\{\mathrm{I}-\frac{\mathrm{I}}{2} q_{2} k_{1}^{2}-\frac{\mathrm{I}}{8} q_{3} k_{1}^{4}-\cdots \text { as above }\right\}\right] \\
+\frac{32 \pi A a}{3(A+a)} n_{1} n_{2}\left[\left(A^{2}+a^{2}\right)(F-E)-2 A a F\right]
\end{gathered}
$$

the modulus of the elliptic integrals being $k_{2}=\frac{2 \sqrt{A a}}{A+a}$

This formula gives an accurate result for equal solenoids of considerable length, but Maxwell's formula (36) is just as accurate and much more convenient.

For short coils neither (46) nor (47) will apply, but for that case as well as other cases Russell's general formula may be used. As the latter is equivalent to (44) it is not here given.

\section{MUTUAL INDUCTANCE OF A SHORT SECONDARY ON THE OUTSIDE OF A LONG PRIMARY}

This is an important case in practice. Havelock ${ }^{41}$ has shown that the mutual inductance of two such solenoids is the same as that of two coils with the same radii and lengths, but with the shorter coil inside. That is, the mutual inductance of a coil of length $l$ and radius $A$ outside of a coil of length $x$ and radius $a$ is the same as 
the mutual inductance of a coil of length $x$ and radius $A$ outside of a coil of length $l$ and radius $a$.

The series formulas already given for the latter case may therefore be applied to the present case directly if the quantities $l$ and $x$, or $l_{1}$ and $l_{2}$, be interchanged.

In Ròiti's formula we put, therefore, $l_{1}=\frac{l-x}{2}$ instead of $\frac{x-l}{2}$. The values of $\rho_{1}$ and $\rho_{2}$ are, however, unchanged and the formula. may be used just as it stands.

Russell's formula being symmetrical in $l_{1}$ and $l_{2}$ requires no change whatever.

In Searle and Airey's formula we have to put

$$
\begin{array}{ll}
d=\sqrt{l^{2}+A^{2}} & L_{2}=3-4 \frac{x^{2}}{a^{2}} \\
X_{2}=3-4 \frac{l^{2}}{A^{2}} & \\
& L_{4}=\frac{5}{2}-10 \frac{x^{2}}{a^{2}}+4 \frac{x^{4}}{a^{4}}
\end{array}
$$

etc.

Cohen's and Nagaoka's formulas apply without change as would be expected.

\section{ROSA'S FORMULAS FOR SINGLE LAYER COILS OF EQUAL RADII}

The mutual inductance of two coaxial single layer coils of equal radii is given by the following expression:

$$
\frac{M}{N_{1} N_{2}}=M_{0}+\Delta M
$$

where $M_{0}$ is the mutual inductance of the two parallel circles at the centers of the coils and $\Delta M$ is given by the following expression: ${ }^{42}$ 


$$
\begin{aligned}
& \Delta M=4 \pi a\left[\frac { \mathrm { I } } { 2 4 } \frac { b _ { 1 } { } ^ { 2 } + b _ { 2 } ^ { 2 } } { d ^ { 2 } } \left\{\mathrm{I}+\frac{3}{8} \frac{d^{2}}{a^{2}}\left(\log \frac{8 a}{d}-\frac{\mathrm{II}}{6}\right)-\frac{45}{25^{6}} \frac{d^{4}}{a^{4}}\left(\log \frac{8 a}{d}-\frac{97}{60}\right)\right.\right. \\
& \left.+\frac{\mathrm{I0} 50}{\mathrm{I}^{2} 8^{2}} \frac{d^{6}}{a^{6}}\left(\log \frac{8 a}{d}-\frac{54}{35}\right)-\frac{44 \mathrm{IOO}}{\mathrm{I}_{28} 8^{3}} \frac{d^{8}}{a^{8}}\left(\log \frac{8 a}{d}-\frac{3793}{2520}\right)+\cdots\right\} \\
& +\frac{\left(b_{1}{ }^{4}+b_{2}{ }^{4}+\frac{\mathrm{IO}}{3} b_{1}{ }^{2} b_{2}{ }^{2}\right)}{3^{20} d^{4}}\left\{\mathrm{I}+\frac{\mathrm{I}}{\mathrm{I} 6} \frac{d^{2}}{a^{2}}-\frac{\mathrm{I} 5}{25^{6}} \frac{d^{4}}{a^{4}}\left(\log \frac{8 a}{d}-\frac{\mathrm{I} 87}{60}\right)\right. \\
& \left.+\frac{2100}{128^{2}} \frac{d^{6}}{a^{6}}\left(\log \frac{8 a}{d}-\frac{893}{420}\right)-\ldots .\right\} \\
& +\frac{b_{1}{ }^{6}+b_{2}{ }^{6}+7\left(b_{1}{ }^{4} b_{2}{ }^{2}+b_{1}{ }^{2} b_{2}^{4}\right)}{2688 d^{6}}\left\{\mathrm{I}+\frac{3}{\mathrm{I} 6 \mathrm{o}} \frac{d^{2}}{a^{2}}-\frac{3}{1024} \frac{d^{4}}{a^{4}}+\cdots\right\} \\
& \left.+\frac{\left(b_{1}^{8}+b_{2}{ }^{8}\right)+\mathrm{I} 2\left(b_{1}{ }^{8} b_{2}{ }^{2}+b_{1}{ }^{2} b_{8}{ }^{6}\right)+\frac{\mathrm{I} 26}{5} b_{1}{ }^{4} b_{2}^{4}}{\mathrm{I} 843^{2} d^{8}}\left\{\mathrm{I}+\frac{\mathrm{I}}{\mathrm{II} 2} \frac{d^{8} a^{8}}{}+\cdots\right\}\right]
\end{aligned}
$$

For coils of equal breadth and equal radii (Fig. $2 \mathrm{I}) b_{1}=b_{2}=b$ and we may write the equation (48) as follows:

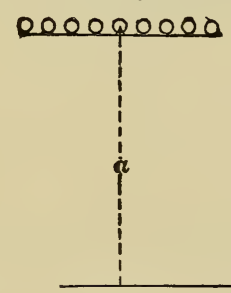

\section{0}

\section{a}

Fig. 21

$$
\begin{aligned}
\Delta M= & 4 \pi a\left[\frac{b^{2}}{\mathrm{I} 2 d^{2}} \mid \mathrm{I}+\frac{3}{8} \frac{d^{2}}{a^{2}}\left(\log \frac{8 a}{d}-\frac{\mathrm{II}}{6}\right)-\frac{45}{256} \frac{d^{4}}{a^{4}}\left(\log \frac{8 a}{d}-\frac{97}{6 \mathrm{O}}\right)\right. \\
& \left.+\frac{\mathrm{IO} 50}{\mathrm{I} 28^{2}} \frac{d^{6}}{a^{6}}\left(\log \frac{8 a}{d}-\frac{54}{35}\right)-\frac{44 \mathrm{IOO}}{\mathrm{I} 28^{3}} \frac{d^{8}}{a^{8}}\left(\log \frac{8 a}{d}-\frac{3793}{2520}\right)+\cdots\right\} \\
+ & \frac{\mathrm{I}}{6 \mathrm{O}} \frac{b^{4}}{d^{4}}\left\{\mathrm{I}+\frac{\mathrm{I}}{\mathrm{I} 6} \frac{d^{2}}{a^{2}}-\frac{\mathrm{I} 5}{25^{6}} \frac{d^{4}}{a^{4}}\left(\log \frac{8 a}{d}-\frac{\mathrm{I} 87}{60}\right)\right. \\
& \left.+\frac{2 \mathrm{IOO}}{\overline{\mathrm{I} 28^{2}}} \frac{d^{6}}{a^{6}}\left(\log \frac{8 a}{d}-\frac{893}{420}\right)-\cdots\right\} \\
+ & \left.\frac{\mathrm{I}}{\mathrm{I} 68} \frac{b^{6}}{d^{6}}\left\{\mathrm{I}+\frac{3}{\mathrm{I} 60} \frac{d^{2}}{a^{2}}-\frac{3}{\mathrm{IO} 24} \frac{d^{4}}{a^{4}}+\cdots\right\}+\frac{\mathrm{I}}{360} \frac{b^{8}}{d^{8}}\left\{\mathrm{I}+\frac{\mathrm{I}}{\mathrm{II} 2} \frac{d^{2}}{a^{2}}-\cdots\right\}\right]
\end{aligned}
$$


This expression will give a very accurate value of $\Delta M$ for two coils not nearer together than their breadth if $a$ is considerably greater than $b$, the breadth of the coil.

For coils which are not so near together the Rosa-Weinstein formula $^{43}$ may be used.

$$
\Delta M=4 \pi a \sin \gamma[(F-E) P+E Q]
$$

where

$$
\begin{aligned}
& P=\frac{\cos ^{2} \gamma}{24 d^{2}}\left[\alpha_{1}-\alpha_{3}-3 \alpha_{3} \cos ^{2} \gamma+8 \alpha_{3} \cos ^{4} \gamma\right] \\
& Q=\frac{\sin ^{2} \gamma}{24 d^{2}}\left[\alpha_{1}+2 \alpha_{3}+3 \alpha_{3} \cos ^{8} \gamma+8 \alpha_{3} \cos ^{4} \gamma\right] \\
& \alpha_{1}=\left(b_{1}{ }^{2}+b_{2}{ }^{2}\right) \\
& \alpha_{3}=\frac{3\left(b_{1}^{4}+b_{2}^{4}\right)+10 b_{1}^{2} b_{2}{ }^{2}}{80 d^{2}} \\
& \sin ^{2} \gamma=\frac{4 a^{2}}{4 a^{2}+d^{2}} \\
& \cos ^{2} \gamma=\frac{d^{2}}{4 a^{2}+d^{2}}
\end{aligned}
$$

and $F$ and $E$ are the complete elliptic integrals of the first and second kinds with modulus $k=\sin \gamma$.

When the coils have equal breadth $b_{1}=b_{2}=b$ and $\alpha_{1}=b^{2}, \alpha_{3}=\frac{b^{4}}{5^{d^{2}}}$.

If the lengths of the coils are not very small in comparison with $d$ a greater precision may be attained by adding to (50) the last two terms of (48) or (49) which depend on differentials of the sixth and eighth order.

\section{MUTUAL INDUCTANCE BY MEANS OF SELF-INDUCTANCE FORMULA}

The mutual inductance of two coils having the same radii and the same number of turns per unit of length may be calculated with great accuracy from a knowledge of several self-inductances.

If the two coils be designated as $\mathrm{A}$ and $\mathrm{B}$ and a coil $\mathrm{C}$ having the same radius and number of turns per unit length be imagined to exactly fill up the space between $A$ and $B$, the self-inductance of coils $A, B$ and $C$ in series will be

$$
L_{\mathrm{ABC}}=L_{\mathrm{A}}+L_{\mathrm{B}}+L_{\mathrm{C}}+2 M_{\mathrm{AC}}+2 M_{\mathrm{BC}}+2 M_{\mathrm{AB}}
$$


Similarly the self-inductances of the coils $\mathrm{A}$ and $\mathrm{C}$ in series, and of $\mathrm{B}$ and $\mathrm{C}$ in series are given by the equations.

$$
\begin{aligned}
& L_{\mathrm{AC}}=L_{\mathrm{A}}+L_{\mathrm{C}}+2 M_{\mathrm{AC}} \\
& L_{\mathrm{BC}}=L_{\mathrm{B}}+L_{\mathrm{C}}+2 M_{\mathrm{BC}}
\end{aligned}
$$

Eliminating $M_{\mathrm{AC}}$ and $M_{\mathrm{BC}}$ in the equation above we find

$$
2 M_{\mathrm{AB}}=\left(L_{\mathrm{ABC}}+L_{\mathrm{C}}\right)-\left(L_{\mathrm{AC}}+L_{\mathrm{BC}}\right)
$$

The self-inductances may be calculated with all the accuracy desired by Lorenz's or Nagaoka's formulas. Formula (5I) is of especial value in testing new formulas and in the case where the two coils are in contact. In the latter case the formula becomes

$$
2 M_{\mathrm{AB}}=L_{\mathrm{AB}}-\left(L_{\mathrm{A}}+L_{\mathrm{B}}\right)
$$

\section{OTHER FORMULAS}

Himstedt has given several formulas for different cases of coaxial solenoids. The first ${ }^{44}$ is for the case of a short secondary on the outside of a long primary. The formula is very complicated, and the calculation tedious. The formulas of Roiti and Searle and Airey may be used to much better advantage.

Himstedt's second expression is for the case of two coaxial solenoids not concentric, the distance between their mean planes having any value; the radius of one is supposed to be considerably smaller than the other. This also is a very complicated formula, involving second and fourth derivatives of expressions containing the elliptic integrals $F$ and $E$. Gray's general equation is much simpler to calculate. This is not, however, an important case in practice, and we do not therefore give Himstedt's equation. Himstedt's third equation is general and applies to two coaxial solenoids of nearly equal or very different radii, which may or may not be concentric. This expression of Himstedt's consists of four terms, each of which is a somewhat complicated expression involving both complete and incomplete elliptic integrals. A less inconvenient general expression for $M$ in elliptic integrals is given above (44).

Havelock ${ }^{45}$ gave a formula for the mutual inductance of two coaxial, concentric solenoids, which resembles somewhat the formula

${ }^{44}$ Wied. Annalen, 26, p. 55 I ; 1885.

${ }_{45}^{4}$ Phil. Mag., 15, p. 342 ; 1908. 
of Ròiti. It is, however, not so convergent as the latter, except when one coil is very short in comparison with the other.

After the present work had gone to press a valuable article appeared $^{45 a}$ by Olshausen, in which the author derived a general absolute expression for the mutual inductance of two coaxial solenoids. Adopting the same nomenclature as in Nagaoka's formula (45), the integral $I$ is in this case given by

$I=m^{\frac{3}{2}} \sqrt{2 A a}\left[\left(\frac{g_{2}}{6}-\mathbf{p}^{2} v\right) \omega_{1}+\mathbf{p} v \cdot \eta_{1}+\frac{\mathbf{p}^{\prime} v}{2}\left\{\eta_{1} v-\omega_{1} \frac{\sigma_{1}}{\sigma}(v)+n \pi \imath\right\}\right] \quad[52 a]$

Here $m$ is a parameter, which is to be arbitrarily assigned, and consequently $(52 a)$ may be put into various special forms depending on the value assumed for $m$. The integer $n$, which may be positive or negative, enters because of the many-valuedness of a logarithm, and is to be found from the equation defining $v$.

If we place $m=\left(\frac{2}{A a}\right)^{\frac{1}{3}}$ and let $n=0$, the result is Nagaoka's equation (45). The author shows further that by expressing the quantities in (52a) in terms of the elliptic integrals of Legendre, Cohen's absolute formula (44) may be shown to be a special case of the general equation $(52 a)$.

As a third example, the author shows that the absolute formula of Kirchhoff, published for the first time by Coffin ${ }^{45 b}$ in a form subsequently shown by Cohen ${ }^{450}$ to be in error, is included in $(52 a)$, and the correct expression is given in Olshausen's equation (38).

Olshausen showed further that if the value $\frac{(A+a)^{2}+c^{2}}{2 A a}$ be assigned to $m$, the expressions for some of the auxiliary quantities become very simple. For the details of calculation as arranged by him we refer to the original article.

\section{CHOICE OF FORMULAS}

I. Coaxial solenoids, not concentric.-(a) For the general case, if the greatest precision is required, Nagaoka's absolute formula (45) should be used. Since, however, the mutual inductance of such 
coils will not in general be needed with extreme accuracy, it will usually be found sufficient to apply Gray's formula (40), taking the precaution to determine by a rough preliminary calculation, whether or not the terms of higher order will have an appreciable effect in the case at hand. For this purpose Table XVIII will be found of material aid.

If the convergence is not satisfactory, or if more than three or four terms must be calculated, it will be found advantageous to subdivide one or both of the coils, and to apply Gray's formula to the calculation of the mutual inductance of the several pairs of sections; for these the convergence will be more rapid. For example, if coil $\mathrm{A}$ be divided into two parts, $\mathrm{C}$ and $\mathrm{D}$, and the coil $\mathrm{B}$ into sections $\mathrm{E}$ and $\mathrm{F}$, then the mutual inductance of $\mathrm{A}$ or $\mathrm{B}$ will be given by the relation

$$
M_{\mathrm{AB}}=M_{\mathrm{CE}}+M_{\mathrm{CF}}+M_{\mathrm{DE}}+M_{\mathrm{DF}}
$$

It may be stated as a general criterion for the rapid convergence of Gray's formula, that the distance between the coils should be great relatively to the radii, and that the coils should not be very long. With long coils it is necessary to carry the subdivision further than with short coils, with a corresponding increase in the number of terms to be calculated, but even then the labor will generally be much less than in using Nagaoka's formula.

If the coils be relatively far apart, and great precision is not desired, the formula of quadratures (23) may be adapted to this case, by making the radial dimension of the cross section of the coils in Fig. 4 equal to zero. We have then

$$
M_{1}=M_{3}=M_{5}=M_{7}=M_{0}
$$

and the formula of quadratures becomes

$$
M=\frac{\mathrm{I}}{6}\left(2 M_{0}+M_{2}+M_{4}+M_{6}+M_{8}\right)
$$

It is, therefore, only necessary to calculate, by an appropriate formula or formulas, the mutual inductances of the five pairs of circles, and to take the weighted mean indicated. This formula is more accurate, the shorter the axial lengths of the solenoids in comparison with their distance apart, and the process of subdivision above described will be, in general, necessary. Gray's formula is, however, to be preferred. 
(b) An important case in practice is that of solenoids of equal radii. If the coils be in contact or very near together the formulas $\left(5^{2}\right)$ or $\left(5^{I}\right)$, respectively, should be employed.

If the solenoids be separated so that the distance between their medial planes is greater than the axial length of either, the mutual inductance may be calculated from the mutual inductance of the two circles at their centers, a correction being applied to take account of the lengths of the coils. For this purpose formula (48) should be used for coils relatively near together and (50) for coils farther apart. The corresponding formulas, for coils of equal radii and equal length are (49) and (50).

2. Coaxial, concentric solenoids of equal length.-If the solenoids be long relatively to their radii, Havelock's formula (38) will be found to be very accurate. Maxwell's formula (36), however, is applicable to both long and short solenoids, provided the radii are not too nearly equal, and should be given the preference, using Havelock's, when desired, as a check on the result. It may'be necessary in rare cases to use the absolute formulas of Nagaoka or Cohen. One should also bear in mind that Ròiti's and Searle and Airey's formulas also hold for equal length solenoids, and may be used in checking the results.

3. Coaxial, concentric solenoids-Inner coil the shorter.-For relatively long coils Ròiti's formula (39) will give very accurate values, whatever the length of the inner solenoid, provided the radius of the inner coil is not closely equal to that of the outer. Ròiti's formula is also applicable to short solenoids in case the inner radius is considerably smaller than the outer. For short solenoids, however, Searle and Airey's formula (43) is preferable, and gives a very rapidly converging value unless the inner radius be nearly equal to the outer. Russell's formula (46) is most convergent for long solenoids, of which the inner one is a good deal shorter than the outer one.

In those cases for which none of the above formulas converge rapidly, and great precision is desired, Nagaoka's or Cohen's absolute formula should be used.

4. Coaxial, concentric solenoids-Outer coil the shorter.-The formulas of the preceding section are to be used interchanging $x$ and $l$, or $l_{1}$ and $l_{2}$ as the case may be. The formulas of Ròiti, 
Russell, Cohen, and Nagaoka are unchanged in form; Searle and Airey's is slightly changed as regards the coefficients $L$ and $X$.

Usually, it will be found that more than one formula will apply to a given case. The advantage of such a check can not be overestimated.

For illustrations and tests of the above formulas, see examples 34-47.

In taking the dimensions of coils where an accurate value of the mutual inductance is sought it should be borne in mind that the above formulas have been derived on the supposition that the current is uniformly distributed over the length of the coaxial solenoids; in other words, these formulas are all current-sheet formulas. Hence, for coils made up of many turns of wire we must meet the conditions imposed by current-sheet formulas. In calculating self-inductances, this requires an accurate determination of the size of the wire and of the distance between the axes of successive wires, from which we can calculate two correction terms to be combined with the value of $L$ given by the current-sheet formulas. ${ }^{46}$

In the case of mutual inductances, however, there are no correction terms to calculate; but we must take the dimensions of the current sheets that are equivalent to the coils of wire; that is, the radius of each coil will be the mean distance to the center of the wire, and the length of each will be the over-all length, including the insulation, when the coil is wound of insulated wire in contact, or the length from center to center of a winding of $n+\mathrm{I}$ turns, where $n$ is the whole number of turns used. ${ }^{47}$ It is also very important that the winding on both coils shall be uniform, ${ }^{48}$ and that the leads shall be brought out so that there shall be no mutual inductance due to them.

The mutual inductance will of course be different at high frequencies from its value at low frequencies. We assume, however, that for all purposes for which an extremely acturate mutual inductance is desired the frequency of the current would be low, say,

${ }^{46}$ Rosa, this Bulletin, 2, p. I8I; I906.

${ }^{47}$ Rosa, this Bulletin, 2, p. I6I, I906; and 3, p. I; 1907.

${ }^{48}$ Searle and Airey, Electrician (London), 56, p. 318; 1905. 
not more than a few hundred per second. If the value at very high frequency is desired the coil should be wound with stranded wire, each strand of which is separately insulated.

EXAMPLES ILLUSTRATING THE FORMULAS FOR THE MUTUAL INDUCTANCE OF COAXIAL SOLENOIDS

EXAMPLE 34. MAXWELL'S FORMULA (36) AND COHEN'S (44)

Two solenoids, Fig. 22, of equal length, $200 \mathrm{~cm}$, each wound with a single layer coil.

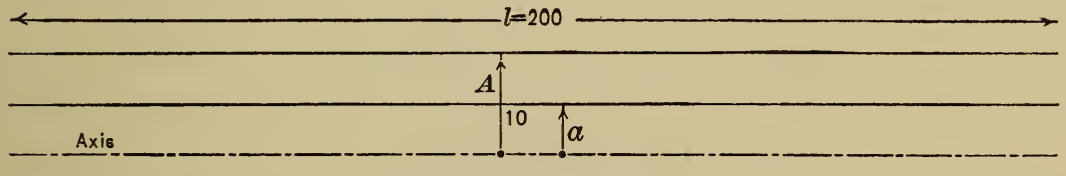

Fig. 22

$$
\begin{aligned}
& A=\text { IO }=\text { radius of outer. } \\
& a=5=\text { radius of inner. }
\end{aligned}
$$

Substituting in (36) for $\alpha$ we have the following:

$$
\begin{aligned}
\alpha & =0.487508-\frac{\mathrm{I}}{\mathrm{I} 6} \frac{a^{2}}{A^{2}}(0.999875)-\frac{\mathrm{I}}{64} \frac{a^{4}}{A^{4}}(0.50000 \mathrm{I})-\frac{35}{2048} \frac{a^{6}}{A^{6}}\left(\frac{\mathrm{I}}{7}\right) \\
& =0.487508-0.015623-0.000488-0.000038 \\
& =0.47 \mathrm{I} 359 \\
\therefore M & =4 \pi^{2} a^{2} n^{2}(200-9.427 \mathrm{I} 8) \\
M & =19057.28 \pi^{2} n^{2}
\end{aligned}
$$

If $n=$ Io turns per $\mathrm{cm}, M=\frac{100 \pi^{2} \times 19057.28}{10^{9}}$ henry

$$
=0.01880878 \text { henry. }
$$

The effect of the next term in the series for $\alpha$ beyond those calculated is to raise the value of $M$ by only one part in five million.

By the approximate formula of Maxwell (37)

$$
\begin{gathered}
\begin{aligned}
2 \alpha=\mathrm{I} & -\frac{\mathrm{I}}{8 \times 4}-\frac{\mathrm{I}}{64 \times \mathrm{I} 6}-\frac{\mathrm{I}}{\mathrm{I02} 4 \times 64}-\cdots \\
& =0.96773 \\
\therefore M & =0.018784 \text { henry. }
\end{aligned} \\
21674^{\circ}-\mathrm{I} 2-6
\end{gathered}
$$


This example by Heaviside's extension of Maxwell's formula (see p. 55) has exactly the same value of $M$; that is, the added terms do not amount to as much as a millionth of a henry in this particular case.

To show that the result by Maxwell's formula (36) is very accurate for this case we may now calculate $M$ by Cohen's absolute formula:

$$
M=4 \pi n^{2}\left(V-V_{1}\right)
$$

Substituting in (44) for $V$ we have the following terms:

$$
\begin{aligned}
V & =7863.79+4200532.04-4 \mathrm{I} 69106.25-2356 \mathrm{I} .95 \\
& =\mathrm{I} 5727.63 \\
V_{1} & =\mathrm{I} 392.18-632.16=760.02 \\
\therefore M & =4 \pi n^{2}(\mathrm{I} 5727.63-760.02)=4 \pi n^{2}(14967.6 \mathrm{I}) \\
& =\mathrm{I} 9057.36 \pi^{2} n^{2} \\
M & =0.01880886 \text { henry. }
\end{aligned}
$$

This agrees with the result by Maxwell's formula to within five parts in a million, the value by Maxwell's formula being more nearly correct, as is shown in the next example.

The example by Cohen's formula illustrates the disadvantage of that formula for numerical calculations. Aside from the fact that it is complicated, and involves the use of both complete and incomplete elliptic integrals, the value of $M$ depends on the difference between very large positive and negative terms, so that in order to get a value of $M$ correct to one part in one hundred thousand it is necessary in the above example to calculate the large terms to one part in twenty-five million. As a means of testing other formulas, however, this absolute formula is of great value.

\section{EXAMPLE 35. HAVELOCK'S FORMULA (38)}

We will take the same problem as in the preceding example:

$$
\begin{aligned}
& a=5 \quad A=\text { I0 } \quad l=200 \\
& \frac{\mathrm{I}}{2}-\frac{\mathrm{I}}{\mathrm{I} 6} \frac{a^{2}}{A^{2}}=0.484375 \\
& \text {-. } \frac{\mathrm{I}}{\mathrm{I} 28} \frac{a^{4}}{A^{4}}=-0.000488
\end{aligned}
$$




$$
\begin{array}{r}
-\frac{5}{2048} \frac{a^{6}}{A^{6}}=-0.000038 \\
-\frac{\mathrm{I}}{4} \frac{A}{l}=-0.012500 \\
\frac{\mathrm{I}}{\mathrm{I} 6}\left(\mathrm{I}+\frac{a^{2}}{A^{2}}\right) \frac{A^{3}}{l^{3}}=\frac{+0.000010}{\mathrm{Sum}}=\beta=\frac{0.47 \mathrm{I} 359}{}
\end{array}
$$

which is exactly the same as the value of $\alpha$ found by Maxwell's formula in the preceding example. The value of the mutual inductance agrees, therefore, exactly to seven significant figures with the value given by Maxwell's formula. For this example, accordingly, we see that Maxwell's and Havelock's formulas give a more accurate value than Cohen's formula, unless the quantities in the latter are carried out to a greater number of places of decimals. This was pointed out by Havelock. ${ }^{49}$

EXAMPLE 36. MAXWELL'S FORMULA (36). FOR EQUAL SHORT SOLENOIDS

$$
\begin{aligned}
& a=5 \quad A=\text { IO } \quad l=2 \\
& r=\sqrt{104} \quad \frac{A}{r}=0.9805808 \quad \frac{a}{A}=\frac{I}{2} \\
& \frac{A-r+l}{2 A}=0.09009805 \\
& -\frac{\mathrm{I}}{\mathrm{I} 6} \frac{a^{2}}{A^{2}}\left(\mathrm{I}-\frac{A^{3}}{r^{3}}\right)=-0.0008927 \mathrm{I} \\
& -\frac{\mathrm{I}}{64} \frac{a^{4}}{A^{4}}\left(\frac{\mathrm{I}}{2}+2 \frac{A^{5}}{r^{5}}-\frac{5}{2} \frac{A^{7}}{r^{7}}\right)=-0.000 \mathrm{r} 3073 \\
& -\frac{35}{2048}(0.080378) \frac{a^{6}}{A^{6}}=-0.00002146 \\
& -\frac{63}{2 . \mathrm{I} 28^{2}}(0.5079) \frac{a^{8}}{A^{8}}=-0.0000038 \mathrm{I} \\
& -\frac{23^{I}}{5^{I^{2}}} \quad(0.788) \frac{a^{10}}{A^{10}}=-0.00000068 \\
& -\frac{429}{2 . \overline{\mathrm{IO}}^{2}} \quad(2.43) \quad \frac{a^{12}}{A^{12}}=-0.00000012 \\
& \mathrm{Sum}=\alpha=0.08904854
\end{aligned}
$$




$$
\begin{aligned}
2 A \alpha & =\quad 1.7809708 \\
l-2 A \alpha & =0.2190292 \\
\frac{\therefore M}{4 \pi n_{1} n_{2}} & =\quad
\end{aligned}
$$

The formula is not so favorable in this case as for long coils, since the quantity $2 A \alpha$ is nearly equal to $l$. Further, the quantities involved in the parentheses are rather large, although their sum is in only one case greater than unity. There is, however, no diffculty in obtaining these factors with all the accuracy required. We have carried out the calculation with this formula further than would in practice be desired, in order to test the formula. We find that to get the same order of accuracy by Searle and Airey's formula terms including the product $X_{12} L_{14}$ must be calculated. The result found was

$$
\frac{M}{4 \pi n_{1} n_{2}}=\mathrm{I} 7.20252
$$

or only one part in two million different. We have also calculated the mutual inductance of these coils by Nagaoka's formula and obtained a value not very different, but this is a very unfavorable case for this formula, no great accuracy being obtainable using seven-place logarithms.

EXAMPLE 37. RÒITI'S FORMULA (39) COMPARED WITH SEARLE AND AIREY'S (43)

We will now calculate the example, Fig. 23 (originally given by Searle and Airey ${ }^{50}$ ), by Ròiti's formula, and also by the formula of Searle and Airey.

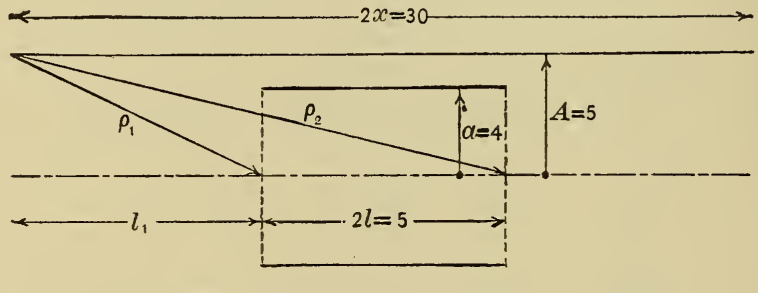

Fig. 23

${ }^{50}$ Electrician (London), 56, p. 319; 1905. 


$$
\begin{aligned}
& 30 \mathrm{~cm}=\text { length of outer solenoid. }
\end{aligned}
$$

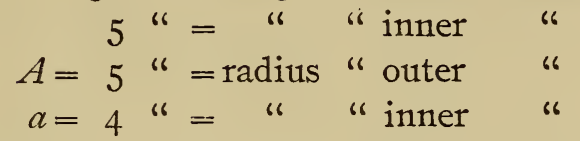

$$
\begin{aligned}
& N_{1}=300 \text { turns } \therefore n_{1}=\frac{300}{30}=\text { IO per cm } \\
& N_{2}=200 \quad \text { " } \quad n_{2}=\frac{200}{5}=40 \text { per } \mathrm{cm} \\
& l_{1}=\mathrm{I} 2.5 \\
& l_{2}=\mathrm{I} 7 \cdot 5 \\
& \rho_{1}=\sqrt{\mathrm{I2.5^{2 } + 2 5}}=\text { I3.4629I2 } \\
& \rho_{2}=\sqrt{I^{I 7 \cdot 5^{2}}+25}=\underline{I 8.200275} \\
& \therefore \rho_{2}-\rho_{1}=4.737363 \\
& \rho_{1}+\rho_{2}=3 \mathrm{I} .663 \mathrm{I} 87
\end{aligned}
$$

It is more accurate to calculate $\left(\rho_{2}-\rho_{1}\right)$ by the formula

$$
\rho_{2}-\rho_{1}=\frac{x l}{\rho_{1}+\rho_{2}} .
$$

This gives $\left(\rho_{2}-\rho_{1}\right)=4.7373620$, which value will be used in the calculation of $M$.

$$
\begin{aligned}
& \rho_{2}-\rho_{1}=4.7373620 \\
& \frac{A^{2} a^{2}}{8}\left(\frac{\mathrm{I}}{\rho_{1}^{3}}-\frac{\mathrm{I}}{\rho_{2}^{3}}\right)=+.0121975 \\
& -\frac{A^{4} a^{2}}{I 6}\left(\frac{I}{\rho_{1}^{5}}-\frac{I}{\rho_{2}^{5}}\right)=-.000704 I \\
& \frac{5}{64} A^{4} a^{4}\left(\mathrm{I}+\frac{\mathrm{I}}{2} \frac{a^{2}}{A^{2}}\right)\left(\frac{\mathrm{I}}{\rho_{1}^{7}}-\frac{\mathrm{I}}{\rho_{2}^{7}}\right)=+.000 \mathrm{I} 808 \\
& -\frac{35 A^{4} a^{6}}{256}\left(\mathrm{I}+\frac{\mathrm{I}}{5} \frac{a^{2}}{A^{2}}\right)\left(\frac{\mathrm{I}}{\rho_{1}{ }^{9}}-\frac{\mathrm{I}}{\rho_{2}{ }^{9}}\right)=-.0000254 \\
& +\frac{\mathrm{IO} 5 A^{6} a^{6}}{\mathrm{IO} 24}\left(\mathrm{I}+\frac{9}{5} \frac{a^{2}}{A^{2}}+\frac{\mathrm{I}}{5} \frac{a^{4}}{A^{4}}\right)\left(\frac{\mathrm{I}}{\rho_{1}^{11}}-\frac{\mathrm{I}}{\rho_{2}{ }^{11}}\right)=+.0000054 \\
& -\frac{693 A^{6} a^{8}}{2048}\left(I+\frac{2}{3} \frac{a^{2}}{A^{2}}+\frac{I}{2 I} \frac{a^{4}}{A^{4}}\right)\left(\frac{I}{\rho_{1}{ }^{13}}-\frac{I}{\rho_{2}{ }^{13}}\right)=-.00000 I 0 \\
& \frac{3003 A^{8} a^{8}}{\mathrm{I}^{6} 34}\left(\mathrm{I}+4 \frac{a^{2}}{A^{2}}+\frac{\text { IO }}{7} \frac{a^{4}}{A^{4}} \cdot \frac{\mathrm{I}}{\mathrm{I} 4} \frac{a^{6}}{A^{6}}\right)\left(\frac{\mathrm{I}}{\rho_{1}{ }^{15}}-\frac{\mathrm{I}}{\rho_{2}{ }^{15}}\right)=+.0000002 \\
& \text { Sum }=\overline{4.7490149}
\end{aligned}
$$




$$
\begin{aligned}
4 \pi^{2} a^{2} n_{1} n_{2} & =25600 \pi^{2} \\
\therefore M & =\frac{25600 \pi^{2} \times 4.7490149}{10^{9}} \text { henry }
\end{aligned}
$$

or $M=0.0011998950$ henry.

The sum of the next two terms in the series is equal to about one part in ten million. The value of the mutual inductance is therefore given with great precision by this formula. If the inner radius had been relatively smaller, the convergence would have been more rapid. We have, however, carried the computation much further than would in practice be necessary.

Calculating the same problem by Searle and Airey's formula we have

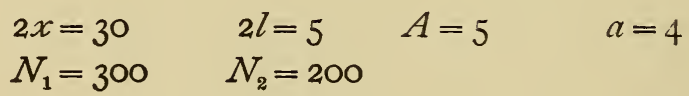

$$
\begin{aligned}
& d=\sqrt{250} \quad \frac{A^{2} a^{2}}{d^{4}}=\frac{4}{625} \\
& X_{2}=-33.00 \quad L_{2}=1.4375^{\circ} \\
& X_{4}=236.5 \quad L_{4}=-0.7959 \\
& X_{6}=-\mathrm{I} 370 \quad L_{6}=-\mathrm{I} .7 \mathrm{I} \\
& X_{8}=4869 \quad L_{8}=-0.72 \\
& \mathrm{I}+\frac{A^{2} a^{2}}{d^{4}} \cdot \frac{L_{2}}{8}=\text { I.00II } 500 \\
& \frac{A^{4} a^{4}}{d^{8}} \frac{L_{4} X_{2}}{32}=0.0000344 \\
& \frac{A^{6} a^{6}}{d^{12}} \frac{L_{6} X_{4}}{32}=-0.0000033 \\
& \text { Sum }=\text { I.00II8II } \\
& \frac{2 \pi^{2} a^{2} N_{1} N_{2}}{d}=\text { I } 998480.5 \\
& \therefore M=0.001 \text { r998957 henry. }
\end{aligned}
$$

The terms neglected are less than one part in ten million. The value of the mutual inductance found is only six parts in ten million greater than that found by Ròit's formula, and for this problem the convergence of Searle and Airey's formula is the more satisfactory. 
The same problem by Russell's formula (46) (extended to include six terms in each part of the formula) gives

$$
M=0.001 \mathrm{I} 9989 \text { henry. }
$$

Of the three formulas Searle and Airey's is for this case the most convergent, and Russell's the least convergent. If the ratio of the radii was still more nearly equal to unity, Searle and Airey's formula would still be satisfactory; the convergence of Ròiti's formula would, however, become poorer.

If in the above problem the length $2 l$ of the inner coil be increased without changing the radii, the quantities $L_{2 n}$ in Searle and Airey's formula would become rapidly larger, and the convergence would become poorer. Ròiti's formula also becomes less satisfactory as $l$ is increased. For $2 l=24$, however, Searle and Airey's formula will still give the correct result to about one part in rooooo, but Ròiti's formula in this case converges very slowly. On the other hand, if the radius of the inner coil were smaller in the latter case, Ròiti's formula could be used, but Searle and Airey's would not converge rapidly enough. This is shown in the next example.

EXAMPLE 38. RÒITI'S FORMULA COMPARED WITH SEARLE AND AIREY'S FORMULA. COILS OF NEARLY EQUAL LENGTH

Length of outer solenoid $=30 \mathrm{~cm}$

$$
\begin{array}{ccc}
\text { inner } & =24 & \\
a=2 & A=5 & n_{1}=\text { IO } \quad n_{2}=40
\end{array}
$$

In Ròiti's formula :

$$
\begin{aligned}
\rho_{2}-\rho_{1} & =2 \mathrm{I} .628108 \\
\text { 2d term } & =+0.062447 \\
3 \text { d } " & =-0.003707 \\
4 \text { th } " & =+0.003682 \\
5 \text { th } " & =-0.000724 \\
6 \text { th } & =+0.000501 \\
7 \text { th } " & =-0.000170 \\
8 \text { th } " & =+\frac{0.000159}{21.690296} \\
\text { Sum } & =6400 \pi^{2} \\
4 \pi^{2} a^{2} n_{1} n_{2} & =600137008 \text { henry. } \\
\therefore M & =0.0013
\end{aligned}
$$


In Searle and Airey's formula

$$
\begin{aligned}
& X_{2}=-33 \quad L_{2}=- \text { I } 4 \text { I } \\
& X_{4}=236.5 \quad L_{4}=4826.5 \\
& X_{6}=-\mathrm{I} 370.3 \quad L_{6}=-\mathrm{I} 60036 \\
& X_{8}=4869 \quad L_{8}=5.019 \times 10^{7} \\
& X_{10}=\text { I } 5746 \quad L_{10}=- \text { I.57 I } \times \text { I0 }^{8} \\
& L_{12}=4.483 \times 10^{9} \\
& d^{2}=250 \\
& 2 \mathrm{~d} \text { term }=-0.028200 \\
& 3 \mathrm{~d} "=-0.012742 \\
& 4 \text { th " }=-0.004845 \\
& 5^{\text {th }} "=-0.001409 \\
& \text { 6th " }=-0.00025 \mathrm{I} \\
& 7^{\text {th }} "=+0.000037 \\
& \mathrm{Sum}=\overline{0.95^{2} 590} \\
& \frac{2 \pi^{2} a^{2} N_{1} N_{2}}{d}=\frac{2304000 \pi^{2}}{d} \\
& \therefore M=0.00136999 \text { henry. }
\end{aligned}
$$

\section{I. .000000}

In this case we see that the higher order terms in Roiti's formula arrange themselves in pairs of nearly equal values with opposite signs. The convergence is, therefore, better than appears at first sight, and the terms here neglected do not amount to more than one part in a million in $M$. Searle and Airey's formula does not converge so rapidly, the eighth and still higher order terms being appreciable. If the length of the inner solenoid were made still greater the $L$ coefficients would become even larger than they are here, and the convergence would become unsatisfactory.

\section{EXAMPLE 39. RÒITI'S AND SEARLE AND AIREY'S FORMULAS IN THE} CASE OF SHORT COILS

Length of the outer solenoid $=5 \mathrm{~cm}$

$$
\text { " " " inner " } \begin{gathered}
=2 \\
a=2
\end{gathered}
$$

In Ròiti's formula:

$$
\begin{aligned}
& \rho_{1}=\operatorname{IO} \operatorname{III} 873 \\
& \rho_{2}=\operatorname{I0.594808}
\end{aligned}
$$


which used in the formula $\frac{x l}{\rho_{1}+\rho_{2}}$ give a more accurate value of $\rho_{2}-\rho_{1}$, viz:

$$
\begin{aligned}
& \rho_{2}-\rho_{1}=0.4829359 \\
& 2 \mathrm{~d} \text { term }=0.0063160 \\
& 3 \mathrm{~d} "=-0.0001968 \\
& 4^{\text {th }} "=0.0003286 \\
& 5^{\text {th }} "=-0.0000274 \\
& \text { 6th " }=0.0000250 \\
& 7 \text { th " }=-0.0000035 \\
& \text { 8th " = } 0.0000023 \\
& \text { Sum }=0.4893^{801} \\
& 4 \pi^{2} a^{2}=\quad 16 \pi^{2} \\
& \therefore \frac{M}{n_{1} n_{2}}=77.2798 \mathrm{I}
\end{aligned}
$$

In Searle and Airey's formula

$$
\begin{aligned}
& X_{2}=2.75 \\
& L_{2}=2 \\
& X_{4}=\frac{12 I}{64} \\
& L_{4}=\frac{I}{4} \\
& X_{6}=\frac{\mathrm{I} 203}{\mathrm{IO} 24} \\
& L_{6}=-\frac{I 5}{I 6} \\
& X_{8}=\frac{9265}{16384} \\
& L_{8}=-\frac{77}{64} \\
& d^{2}=\mathrm{I} 06.25 \\
& L_{10}=-\frac{9}{\mathrm{I} 6} \\
& \text { I. } 0000000 \\
& \text { Ist term }=0.008858 \mathrm{I} \\
& 2 \mathrm{~d} "=0.0000270 \\
& 3 \mathrm{~d} \text { " }=-0.0000025 \\
& \text { 4th " }=-0.0000001 \\
& \text { Sum }=\overline{\mathrm{I} .0088825} \\
& \frac{2 \pi^{2} a^{2} N_{1} N_{2}}{d}=\frac{8 \mathrm{o} \pi^{2} n_{1} n_{2}}{d} \\
& \therefore \frac{M}{n_{1} n_{2}}=77.27980
\end{aligned}
$$

The neglected terms in Searle and Airey's forumula are entirely inappreciable. The convergence of Ròiti's formula is not quite so 
good. The sum of the next two terms is such as to reduce $M$ by three units in the last place, but the following terms do not decrease very rapidly. We may evidently regard the use of either formula as entirely justified in the problem. If the radius of the outer coil had been only one-half as great, the lengths of the two coils and the radius of the inner remaining unchanged, the value found by Ròiti's formula would be in error by more than one part in ten thousand; the convergence of Searle and Airey's would, however, be satisfactory in this case. This formula would, on the other hand, be less convergent when the length of the inner coil is nearly as great as that of the outer coil. In general, it will be found that these two formulas between them cover sufficiently well the cases which may arise in practice.

\section{EXAMPLE 40. GRAY'S FORMULA (41) COMPARED WITH RÒITIS (39)}

Let the winding be 20 turns per $\mathrm{cm}$ on each coil (Fig. 24), $n_{1}=n_{2}=20$.

$$
\begin{array}{ll}
A=25 \mathrm{~cm} & N_{1}=n_{1} A \sqrt{3} \\
a=10 \mathrm{~cm} & N_{2}=n_{2} a \sqrt{3}
\end{array} \quad \therefore N_{1} N_{2}=3 n_{1} n_{2} A a
$$

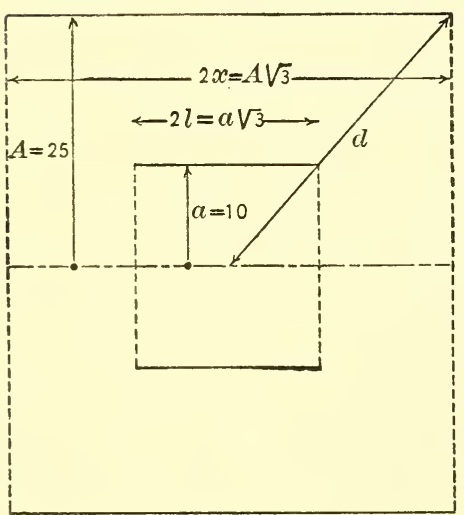

Fig. 24

$$
\begin{gathered}
d=\sqrt{x^{2}+A^{2}}=\frac{-}{2} \sqrt{7} \\
\therefore \quad M=\frac{2 \pi^{2} a^{2} N_{1} N_{2}}{d}=4 \pi^{2} a^{2} n_{1} n_{2}\left[\frac{\overline{3^{a}}}{\sqrt{7}}\right] \\
M=\text {. OI } 79057 \text { henry. }
\end{gathered}
$$

We have also calculated the mutual inductance for these coils by Ròiti's formula (39).

The value is, $M=.017905^{8}$, which is practically identical with the value by Gray's formula.

When $A=25 \mathrm{~cm}$ and $a=10 \mathrm{~cm}, N_{1}=20 A \sqrt{3}=866.025$ and $N_{2}=$ $20 a \sqrt{3}=346.4$. As there must be an integral number of turns, let us suppose the winding is exactly 20 turns per cm on each coil and the lengths therefore $43.3 \mathrm{~cm}$ and $I 7.3 \mathrm{~cm}$, respectively. Then $d=\sqrt{x^{2}+A^{2}}=\sqrt{625+\left(\frac{43 \cdot 3}{2}\right)^{2}}=33.07$ I $5 \mathrm{~cm}$. This does not exactly 
conform to the condition imposed in deriving the simple formula (4I) of Gray used above. Hence (4I) will not be as exact with these slightly altered dimensions, and we must calculate at least one correction term to get an accurate value of $M$.

By Gray's formula ( $4 \mathrm{I}), M=\frac{2 \pi^{2} \mathrm{IOO} \times 866 \times 346}{33.07 \mathrm{I} 5 \times 10^{9}}=.0178842$ henry.

The first correction term in (43) increases this value to .oI 78854 henry.

We will now calculate the mutual inductance of these coils by Ròiti's formula (39) :

$$
\begin{aligned}
& \begin{array}{lll}
A=25 & 2 x=43.3 & l_{1}=\mathrm{I} 3.0 \mathrm{~cm} \\
a=\mathrm{IO} & 2 l=\mathrm{I} 7.3 & l_{2}=30.3 \mathrm{~cm}
\end{array} \\
& M=\frac{4 \pi^{2} a^{2} n_{1} n_{2} \times \mathrm{II} \cdot 32596}{\mathrm{IO}^{9}} \text { henry, } \\
& =.0178853 \text { henry. } \\
& \begin{aligned}
\rho_{1} & =28.17800 \\
\rho_{2} & =\frac{39.282 \mathrm{I} 8}{\text { II.IO4I8 }} \\
\rho_{2}-\rho_{1} & =.22030 \\
\text { 2nd term } & =+ \\
3 \text { rd } & =-.0178 \mathrm{I} \\
4 \text { th } & =+.01952 \\
5 \text { th } & =+.00156 \\
6 \text { th } & =-.00453 \\
7 \text { th } & =+.00274 \\
\text { Sum } & =\frac{\text { II.32596 }}{\text { SI. }}
\end{aligned}
\end{aligned}
$$

This differs from the result by Gray's formula by only I part in I 78000 .

\section{EXAMPLE 41. GRAY'S FORMULA (40) COMPARED WITH NAGAOKA'S} FORMULA (45)

We will next consider a practical problem suggested by Prof. Nasmyth.

$$
\begin{aligned}
& 2 x=20.55 \quad A=6.44 \quad N_{1}=\mathrm{I} 5 \text { turns. } \\
& 2 l=27.3^{8} \quad a=4.435 \quad N_{2}=75 \quad \text { " }
\end{aligned}
$$

The distance between the adjacent ends of the two solenoids was $7.2 \mathrm{~cm}$. From this we find

$$
\begin{aligned}
& n_{1}=0.7296 \text { turns per cm } \\
& n_{2}=2.737 \\
& x_{1}=20.89 \\
& x_{2}=4 \mathrm{I} .44
\end{aligned}
$$

$$
\begin{aligned}
k_{1} K_{1} & =0.042937 \\
k_{3} K_{3} & =0.018274 \\
k_{5} K_{5} & =0.005193 \\
k_{7} K_{7} & =0.001423 \\
k_{9} K_{9} & =0.000116 \\
\mathrm{Sum} & =0.067943 \\
\therefore \mathrm{M} & =1092.3 \mathrm{~cm} .
\end{aligned}
$$


It is evident that the convergence is not rapid enough to give a very precise value for the mutual inductance. We next divide the longer coil $\mathrm{S}$ into two sections $\mathrm{C}$ and $\mathrm{D}$, such that $\mathrm{C}$ has 37 turns and $\mathrm{D}$ has 38 turns, $\mathrm{C}$ being the section nearer the other coil $\mathrm{R}$. The axial lengths of these sections are, respectively, I $3.5 \mathrm{I}$ and $I 3.87 \mathrm{~cm}$. It would be just as well, if not better, to divide the coil into two equal sections of $37 \mathrm{I} / 2$ turns each. The division chosen was for greater convenience in the solution of the same problem by the method of quadratures. (Example 42.)

We now proceed to find $M_{\mathrm{RC}}$ and $M_{\mathrm{RD}}$. The $L$ coefficients are much smaller than before on account of the ratio $\frac{l}{a}$ being now smaller than was previously the case, and the convergence is much more satisfactory. These coefficients would be still smaller if we had divided coil $\mathrm{R}$ instead of $\mathrm{S}$ into two sections, measuring the $x$ 's from the center of $\mathrm{R}$ instead of using $\mathrm{S}$ for the reference coil as is done here. This advantage would, nevertheless, be in large measure offset by the smaller values of the distances $r_{1}$ and $r_{2}$.

We find for $M_{\mathrm{RC}}$

$$
\begin{aligned}
k_{1} K_{1} & =0.048894 \\
k_{3} K_{3} & =0.006520 \\
k_{5} K_{5} & =0.00005 \mathrm{I} \\
\text { Sum } & =0.055465 \\
\therefore M_{\mathrm{RC}} & =89 \mathrm{I} .7 \mathrm{~cm}
\end{aligned}
$$

and for $M_{\mathrm{RD}}$

$$
\begin{aligned}
k_{1} K_{1} & =0.0 \mathrm{II} 549 \\
k_{3} K_{3} & =0.0006 \mathrm{I} 3 \\
k_{5} K_{5} & =0.000004 \\
\text { Sum } & =0.012 \mathrm{I} 66 \\
\therefore M_{\mathrm{RD}} & =195.6 \mathrm{~cm} .
\end{aligned}
$$

Consequently $M=M_{\mathrm{RC}}+M_{\mathrm{RD}}=1087.3 \mathrm{~cm}$.

To test the correctness of this value, the coil $\mathrm{R}$ was divided into two sections $A$ and $B$ ( $B$ being the section nearer to $S$ ), and the four mutual inductances between these sections and the two sections $\mathrm{C}$ and $\mathrm{D}$ of the coil $\mathrm{S}$ were calculated. 


$$
\begin{array}{rccc}
M_{\mathrm{AC}} & M_{\mathrm{BC}} & M_{\mathrm{AD}} & M_{\mathrm{BD}} \\
k_{1} K_{1}=0.012024 & 0.036869 & 0.003893 & 0.007657 \\
k_{3} K_{3}=0.000863 & 0.005654 & 0.00014 \mathrm{I} & 0.00047 \mathrm{I} \\
k_{5} K_{5}=\frac{0.000004}{0.01289 \mathrm{I}} & \frac{0.000047}{0.042570} & \frac{0.00000 \mathrm{I}}{0.004035} & \frac{0.000004}{0.008 \mathrm{I} 32} \\
M_{\mathrm{AC}}=207.2 & M_{\mathrm{BC}}=684.4 & M_{\mathrm{AD}}=64.9 & M_{\mathrm{BD}}=\mathrm{I} 30.7 \\
M=\mathrm{sum}=\mathrm{I0} 87.2 \mathrm{~cm} . &
\end{array}
$$

The only component for which the convergence was not entirely satisfactory was $M_{\mathrm{BC}}$. Here the sections are relatively near together and the coefficients $L$ and $X$ are not very favorable. Accordingly $M_{\mathrm{BC}}$ was calculated by two other methods $(a)$ by dividing $\mathrm{B}$ into two sections, $\mathrm{H}$ and $\mathrm{J}$, and by calculating $M_{\mathrm{HC}}$ and $M_{\mathrm{JC}},(b)$ by dividing $\mathrm{C}$ into two sections, $\mathrm{F}$ and $\mathrm{G}$, and by calculating $M_{\mathrm{BF}}$ and $M_{\mathrm{BG}}$. The first procedure, on aceount of the relatively smaller values of $r_{1}$ and $r_{2}$, did not give a satisfactory degree of convergence. The latter, however, is better, the values found being

$$
\dot{M}_{\mathrm{BC}}=463.8+220.0=683.8
$$

Using this value instead of the above the value of $M$ is 1086.6 $\mathrm{cm}$.

As the final check we have calculated the mutual inductance by Nagaoka's formula (45). The entire calculation has to be carried through for four different values of $c$, viz, 55. I3, 34.58, 27.75, and 7.20. The corresponding values of $I$ are

$$
\begin{aligned}
& I_{1}=60.04 \mathrm{I} 8 \mathrm{O} 2 \\
& I_{2}=38.047638 \\
& I_{3}=30.8 \mathrm{II} 676 \\
& I_{4}=10.333503
\end{aligned}
$$

and $\left(I_{1}+I_{4}\right)-\left(I_{2}+I_{3}\right)=70.375305-68.8593$ I 4

$$
=\mathrm{I} .5^{\mathrm{I}} 599 \mathrm{I}
$$

$$
\therefore M=4 \pi n_{1} n_{2} A a(\mathrm{I} .5 \mathrm{I} 599 \mathrm{I})=1086.55 \mathrm{~cm} \text {. }
$$

An inspection of the various details of the calculation shows that the last figure may be in error by several units, although the utmost precision of which the seven-place logarithms are capable was striven 
for. Of course by carrying the computation of the various quantities to a still greater number of decimal places, the accuracy of the result would be enhanced. Similarly the component values of the mutual inductance by Gray's formula would have been more accurate if we had not stopped with $k_{5} K_{5}$. Since the dimensions of such coils would not ordinarily be obtained with greater precision than the accuracy here attained by Gray's formula, it is evident that the latter is for such cases much to be preferred to Nagaoka's formula, and the same would be true if the number of components were considerably increased. Nagaoka's formula has nevertheless the advantage in checking other formulas.

\section{EXAMPLE 42. FORMULA OF QUADRATURES}

The problem treated in the preceding example may also be solved by the formula of quadratures, using formula (8) for the calculation of the mutual inductance of the various pairs of circles. In general the method is not so accurate as that in the preceding example, and no time is saved. Only the results are here given, together with those by Gray's formula for comparison.

$$
\begin{aligned}
& \text { Single coils Two sections Four sections } \\
& M=964 . \mathrm{I} \quad M_{\mathrm{RC}}=848 . \mathrm{I} \quad M_{\mathrm{AC}}=205.7 \quad M_{\mathrm{BF}}=504.5 \\
& \begin{array}{llll}
M_{\mathrm{RD}}=193.7 & \frac{M_{\mathrm{BC}}=669.5}{M=104 \mathrm{I} .8} & 875.2 & \frac{M_{\mathrm{BG}}=182.2}{M_{\mathrm{RC}}=} \\
M_{\mathrm{BC}}=686.7
\end{array} \\
& M_{\mathrm{AD}}=66.5 \\
& M_{\mathrm{BD}}=\mathrm{I} 30.4 \\
& M_{\mathrm{RD}}=\underline{I 96.9} \\
& M=\overline{\mathrm{IO}} 2 . \mathrm{I}
\end{aligned}
$$

Using the value of $M_{\mathrm{BC}}$ in the last column, $M=\mathrm{I0} 99 \cdot 3$.

By Gray's formula:

$$
\begin{aligned}
& \text { Single coils Two sections Four sections } \\
& M=1092.3 \quad M_{\mathrm{RC}}=89 \mathrm{I} .7 \quad M_{\mathrm{AC}}=207.2 \\
& \frac{M_{\mathrm{RD}}=195.6}{M=1087.3} \quad \frac{M_{\mathrm{BC}}=684.4}{M_{\mathrm{RC}}=} \quad 891.6 \quad \frac{M_{\mathrm{CJ}}=220.0}{M_{\mathrm{BC}}=683.8} \\
& M_{\mathrm{AD}}=64.9 \\
& M_{\mathrm{BD}}=\mathrm{I} 30.7 \\
& M_{\mathrm{RD}}=\frac{\mathrm{I} 95.6}{M=1087.2}
\end{aligned}
$$

Using the value of $M_{\mathrm{BC}}$ in the last column, $M=$ Io86.6. 


\section{EXAMPLE 43. MUTUAL INDUCTANCE OF CONCENTRIC COAXIAL SOLENOIDS BY NAGAOKA'S FORMULA (45)}

$$
\begin{aligned}
2 x & =200 \mathrm{~cm} \\
2 l & =20 \\
A & =\mathrm{I} 5 \\
a & =\mathrm{IO}
\end{aligned}
$$

This pair of coils was used by Cohen ${ }^{51}$ in testing his absolute formula. He gave as the result $M=4 \pi n_{1} n_{2}(62$ r 3.4$)$. The same problem was worked out by Nagaoka ${ }^{52}$ as an illustration of the use of his formula, the value of $M$ found by him being $M=4 \pi n_{1} n_{2}$ (62I3.5I). We have repeated his calculation, which was given only in abbreviated form, and agree substantially with his result, the value found being $M=4 \pi n_{1} n_{2}(6213.52)$. Using seven-place logarithms it is very difficult to be sure of the last place of decimals given here. On the other hand, we find with Ròiti's formula, only three terms being necessary $M=4 \pi n_{1} n_{2}$ (62r3.509), and the same number of terms in Searle and Airey's formula give $M=4 \pi n_{1} n_{2}$ (62I3.5IO) with no uncertainty greater than one unit in the last place given. Olshausen found for the same coils the values $4 \pi n_{1} n_{2}$ (62 I3.77), and $\left.4 \pi n_{1} n_{2}(62)_{3} \cdot 63\right)$, using two methods of calculation in his formulas (2I) and (6I) and with $m=\frac{(A+a)^{2}+c^{2}}{2 a A}$. By the Kirchhoff formula he found $4 \pi n_{1} n_{2}(62$ I2.9). This is, however, an unfavorable case for this formula, since the angles $\phi$ and $\theta$, on which the incomplete integrals $E\left(\varphi, k^{\prime}\right)$ and $F\left(\varphi, k^{\prime}\right)$ depend, are too near $90^{\circ}$ to allow of accurate interpolation in Legendre's tables.

These differing results by the various absolute formulas, which arise from the fact, that in all of them the auxiliary quantities must be calculated with a considerably greater degree of accuracy than that desired in the result, serve to emphasize the advantage of the series formulas. In the great majority of practical cases the values, found by the use of series formulas, are not only obtained with a much smaller expenditure of labor, but are more accurate than when an absolute formula is used.

${ }^{52}$ Jour. Tokyo, Math. Phys. Soc. (2), 4, p. 284; 1908. 
We reproduce below the principal results in the calculation of this problem by Nagaoka's formula.

$$
\begin{aligned}
& A+a=25 \quad A-a=5 \quad A^{2}-a^{2}=125 \\
& (A+a)^{2}=625 \quad(A-a)^{2}=25
\end{aligned}
$$

The argument of $I_{1}=-I_{3}$ is $c=$ IIO; that of $I_{2}=-I_{4}$ is $c=90$.

$$
\begin{aligned}
& \mathrm{C}=110 \\
& \log \alpha=\quad \overline{\mathrm{I}} \cdot 3749796 \\
& \log 2 \text { Aa }=\quad \text { I.8521009 } \\
& P_{1}=\mathrm{p} v-e_{1}=-\mathrm{I} 70.09225 \\
& P_{2}=\mathrm{p} v-e_{2}=+0.3514302 \\
& P_{3}=\mathrm{p} \tau^{\prime}-e_{3}=+8.785755 \mathrm{I} \\
& \mathrm{p} v=\frac{\mathrm{I}}{3}\left(P_{1}+P_{2}+P_{3}\right)=-53.65 \mathrm{I} 687 \\
& e_{2}-e_{3}=8.4343249 \\
& e_{1}-e_{3}=\mathrm{I} 78.878 \mathrm{OI} \\
& e_{1}-e_{2}=\text { I70.44368 } \\
& k^{\prime}=\sqrt{\frac{e_{1}-e_{2}}{e_{1}-e_{3}}}=0.9761398 \\
& \mathrm{I}+\sqrt{k^{\prime}}=\quad \mathrm{I} .9879979 \\
& \log k^{2}=\quad \overline{2} .6734934 \\
& q=0.0030186570 \\
& \log \omega_{1}(\text { ist equat. })=\quad \overline{\mathrm{I}} .0750696 \\
& \text { " }(2 \mathrm{~d} \text { " })=\overline{\mathrm{I}} .0750696 \\
& \text { " }(3 \mathrm{~d} \text { " })=\overline{\mathrm{I}} .0750696 \\
& P_{2}+P_{3}=\text { 9.1 } 37 \text { I } 853 \\
& P_{1}+2 P_{2}=-169.38939 \\
& P_{1}+2 P_{3}=-\mathrm{I} 52.52074 \\
& \frac{P_{1}}{2}\left(P_{2}+P_{3}\right)=-777.08214 \\
& \frac{\left(e_{2}-e_{3}\right)}{6}\left(P_{1}+2 P_{2}\right)=-238.11479 \\
& - \text { Sum }=+ \text { IOI } 5.19693 \\
& \times \omega_{1}=\quad \mathrm{I} 20.67564 \\
& \frac{\mathrm{p} v}{4 \omega_{1}} \frac{\theta_{3}{ }^{\prime \prime}(\mathrm{o})}{\theta_{3}(\mathrm{o})}=26.73272 \\
& \text { Diff. }=I^{\prime}=\quad 93.94292 \\
& \text { (other equat. })=\quad \text { 93.94292 I } \\
& \mathrm{C}=90 \\
& \text { I. } 3749796 \\
& \text { I. } 8521009 \\
& \text { - II } 3.86339 \\
& +\quad 0.3514302 \\
& +8.785755^{1} \\
& \text { - } 34.908733 \\
& 8.4343249 \\
& \text { I } 22.64916 \\
& \text { II } 4.2 \text { I } 483 \\
& 0.9650037 \\
& \text { I. } 982346 \mathrm{x} \\
& \overline{2} .8373857 \\
& 0.00445280 \text { ro } \\
& \text { I.I } 594886 \\
& \text { I.I } 594886 \\
& \text { I.I } 594887 \\
& \text { 9. } 137 \text { I } 853 \\
& \text { - II } 3.16053 \\
& \text { - } 96.29188 \\
& -520.19542 \\
& \text { - I } 59.07205 \\
& 679.26747 \\
& 98.068477 \\
& 21.064838 \\
& 77.003639 \\
& \underline{77.003659}
\end{aligned}
$$




$$
\begin{aligned}
& s=-0.33164947 \quad-0.33084473 \\
& -\frac{s}{q}=\operatorname{Iog} .86656 \\
& \mathrm{I}+2 q^{4} \cos 4 \pi \mathrm{r}=\quad \mathrm{I} .0000042 \quad \mathrm{I} .0000087 \\
& b=109.86702 \quad 74.301000 \\
& \sqrt{\frac{b-I}{b+I}}=\quad 0.99093909 \quad 0.98663068 \\
& 4 q^{2} \sqrt{b^{2}-\mathrm{I}}\left[\mathrm{I}-q^{2}(2 b-\mathrm{I})\right]=\quad 0.0039964 \mathrm{I} \quad 0.00587502 \\
& \mathrm{Sum}=\quad 0.9949355^{\circ} \quad 0.99250570 \\
& I^{\prime \prime}=-35.815107 \quad-29.231710 \\
& \frac{I}{2}=I^{\prime}+I^{\prime \prime}=\quad \text { 58.I278I4 47.77I939 }
\end{aligned}
$$

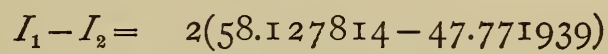

$$
\begin{aligned}
& =2(\text { I0.355875) } \\
& M=4 \pi n_{1} n_{2} A a \cdot 2\left(I_{1}-I_{2}\right) \\
& =4 \pi n_{1} n_{2}(62 \mathrm{I} 3.52)
\end{aligned}
$$

In the calculation of $I$ we have used the second value found for $I^{\prime}$ with $c=$ IIO and the mean of the two values for $I^{\prime}$ with $c=90$.

EXAMPLE 44. SHORT SECONDARY ON THE OUTSIDE OF A LONG

\section{PRIMARY}

Length of primary $=200 \mathrm{~cm}$

" " secondary $=5$

Radius of primary $=4=a$

" " secondary $=5=A$

$$
n_{1}=\mathrm{IO} \quad n_{2}=40
$$

In Ròiti's formula:

$$
\begin{aligned}
\rho_{1} & =97.628 \mathrm{II} \\
\rho_{2} & =\mathrm{I} 02.62 \mathrm{I} 88 \\
\rho_{2}-\rho_{1}=\frac{x l}{\rho_{1}+\rho_{2}} & =4.9937586 \\
\frac{A^{2} a^{2}}{8}\left(\frac{\mathrm{I}}{\rho_{1}^{3}}-\frac{\mathrm{I}}{\rho_{2}^{3}}\right) & =\frac{0.0000075}{\mathrm{Sum}}=\frac{4.993766 \mathrm{I}}{4 \pi^{2} a^{2} n_{1} n_{2}}=25600 \pi^{2} \\
\therefore M & =0.00 \mathrm{I} 26 \mathrm{I} 7342 \text { henry. }
\end{aligned}
$$


In Searle and Airey's formula:

$$
\begin{aligned}
& d^{2}=10025 \quad N_{1} N_{2}=400000 \\
& \frac{l}{A}=20 \quad \frac{x}{a}=\frac{5}{8} \\
& X_{2}=\text { - I } 597 \quad L_{2}=\quad \text { I.4375 } \\
& L_{4}=-0.796
\end{aligned}
$$

\section{I.00000000}

in exact agreement with the above. Both formulas are very rapidly convergent, and give as nearly the same value for $M$ as can be cal-

\begin{tabular}{|c|c|c|c|}
\hline $\begin{array}{l}\text { Ist term } \\
2 \text { d term } \\
\text { 3d term } \\
\text { 4th term }\end{array}$ & \begin{tabular}{rr} 
& \multicolumn{1}{c}{ First } \\
$=$ & 0.088055 \\
$=$ & -0.012694 \\
$=$ & 0.001232 \\
$=$ & -0.000104 \\
\end{tabular} & $\begin{array}{c}\text { Second } \\
\text { o.oI } 562 \\
0.00126 \\
0.00130\end{array}$ & $\begin{array}{r}\text { Third } \\
0.0047 \\
-0.0002\end{array}$ \\
\hline $\mathrm{am}$ & $=0.076489$ & 0.01818 & 0.0045 \\
\hline
\end{tabular}
culated with seven-place logarithms.

EXAMPLE 45. COIIS OF EQUAL RADII NEAR TOGETHER, BY FORMULA (48)

Lengths of the coils $4 \mathrm{~cm}$ and $6 \mathrm{~cm}=b_{1}$ and $b_{2}$ Radius " " " $20 \mathrm{~cm}=a$

Distance between centers Io $\mathrm{cm}=d$

The calculation of the quantities in the parentheses is as follows:

The expression for $\Delta M$ then gives:

$$
\begin{aligned}
& \text { First term }=0.0233240 \\
& \text { Second " }=0.0011047 \\
& \text { Third " }=0.0000973 \\
& \text { Fourth " }=0.0000113 \\
& \text { Sum } \quad=0.0245373
\end{aligned}
$$


The value of $M_{0}$ calculated for two circles of radius $20 \mathrm{~cm}$ and at a distance apart = Io $\mathrm{cm}$ was found by (4) and checked by (I) to be

$$
\begin{aligned}
\frac{M_{0}}{N_{1} N_{2}} & =4 \pi a(0.8853877) \\
\frac{\Delta M_{0}}{N_{1} N_{2}} & =4 \pi a(0.0245373) \\
\therefore \quad \frac{M}{N_{1} N_{2}} & =4 \pi a(0.9099250)
\end{aligned}
$$

This was checked by means of (5I) with the result, (assuming one turn per $\mathrm{cm}$ of the length of the coils)

$$
\begin{aligned}
& L_{\mathrm{ABC}}=4 \pi a(430.339736) \\
& L_{\mathrm{C}}=\frac{4 \pi a(74.324564)}{\text { Sum }}=4 \pi a(504.664300) \\
& L_{\mathrm{AO}}=4 \pi a(194.2 \mathrm{IOI} 35) \\
& L_{\mathrm{BC}}=4 \pi a(266.777705) \\
& \text { Sum }=4 \pi a(460.987840) \\
& \frac{\mathrm{I}}{2} \text { Diff. }=4 \pi a \quad(2 \mathrm{I} .838230)
\end{aligned}
$$

Dividing by 24 , the product of the number of turns assumed in calculating the self-inductances,

$$
\frac{M}{N_{1} N_{2}}=4 \pi a(0.9099264)
$$

which agrees with the value by (48) to about one and a half in a million.

For these coils, therefore, (48) is adequate to give a high degree of precision. If the distance between the same coils were, however, smaller, or if the lengths of the coils were greater the accuracy would not be so great, and it might be necessary to use (5I). The latter, should, however, not be used when (48) converges well, since to get the same accuracy the calculation of the four self-inductances must be carried out to a greater number of decimal places than appear in the value of $M$. For the rapid convergence of (48) the ratios $\frac{b_{1}}{d}, \frac{b_{2}}{d}$ and $\frac{d}{a}$ should all be small.

For the more unfavorable case $b_{1}=6, b_{2}=\mathrm{IO}, d=\mathrm{IO}, \alpha=2 \mathrm{O}$ the value of $\Delta M$ comes out too small by three parts in ten thousand. 


\section{EXAMPLE 46. ROSA-WEINSTEIN FORMULA (50). FOR COILS FARTHER} APART

As a rather unfavorable case we may take

$$
\begin{aligned}
& b_{1}=\mathrm{IO} \quad b_{2}=20 \quad d=50 \quad a=25 \\
& k=\quad \sin \gamma=\frac{50}{\sqrt{5000}}=\frac{I}{\sqrt{2}}=\cos \gamma \\
& \frac{\cos ^{2} \gamma}{24 d^{2}}=\frac{\sin ^{2} \gamma}{24 d^{2}}=\frac{\mathrm{I}}{\mathrm{I} 20000} \\
& \alpha_{1}=500 \\
& -\alpha_{3}=-4.55 \\
& -3 \alpha_{3} \cos ^{2} \gamma=-6.825 \\
& 8 \alpha_{3} \cos ^{4} \gamma=+ \text { 9.10 } \\
& \text { Sum }=497.725 \\
& P=.0041477 \\
& F=\mathrm{I} .854075 \\
& E=\quad \mathrm{I} .350644 \\
& F-E=\quad 0.50343 \mathrm{I} \\
& (F-E) P+E Q=0.0079974 \\
& \therefore \Delta M=1.7766
\end{aligned}
$$

Terms in the 6 th and 8 th differentials $=0.0016$

$$
\mathrm{Sum}=\overline{\mathrm{I} \cdot 7782}
$$

From formula (19) which applies to the two circles at the centers of these coils

$$
\begin{aligned}
M_{0} & =\mathrm{I} .4 \mathrm{I} 8599 \times 25=35.4650 \\
\therefore \frac{M}{N_{1} N_{2}} & =35.4650+\mathrm{I} .7782=37.2432
\end{aligned}
$$

If we calculate the mutual inductance by formula (5I) we find

$$
\begin{array}{rlr}
L_{\mathrm{ABC}} & =3792.226 \mathrm{I} & L_{\mathrm{AC}}=2350.4870 \\
L_{\mathrm{C}} & =\frac{\mathrm{I} 667.7268}{5459.9529} & L_{\mathrm{BC}}=\frac{3062.0405}{54 \mathrm{I} 2.5275} \\
\frac{\mathrm{I}}{2} \text { Diff. }=\quad 23.7 \mathrm{I} 27 &
\end{array}
$$

Dividing by $200=0.1185635=\frac{M}{4 \pi \alpha N_{1} N_{2}}$

$$
\therefore \frac{M}{N_{1} N_{2}}=37.2478
$$


which is more than one in ten thousand greater than the value by (50). If the coils had been shorter and their diameter had been greater than the distance between their medial planes, the quantities $P$ and $Q$ in (50) would have been more convergent and the value of $\Delta M$ would have been more nearly correct. The accuracy here obtained would, however, suffice in many cases.

This formula when applied to the coils in the preceding problem gives a very accurate result viz, $\frac{M}{N_{1} N_{2}}=4 \pi a(0.909932)$, or about six in a million too large. (The terms in the sixth and eight order differentials as calculated by (48) are taken into account in this result.)

The mutual inductance of the coils in this example could also be calculated with a good degree of accuracy by Gray's formula.

\section{EXAMPLE 47. METHOD OF OBTAINING THE DIMENSIONS OF THE EQUIVALENT CURRENT SHEETS}

Suppose it is desired to obtain the mutual inductance of two solenoids, whose measured dimensions are as follows:

Coil I is wound with roo turns of insulated wire of $0.15 \mathrm{~cm}$ covered diameter, the successive turns being in contact. The measured external diameter of the coil is $50.4 \mathrm{~cm}$.

Coil II is wound with 50 turns of bare wire, O.I cm in diameter, in a thread of $2 \mathrm{~mm}$ pitch. The diameter measured over the wire is $10.25 \mathrm{~cm}$.

Then the mean radius of coil $I$, to the center of the wire, is equal to $\frac{I}{2}(50.4-0.15)$ or $25.125 \mathrm{~cm}$. The length of the equivalent current sheet will be the distance between the center of the first and the one hundred and first wire, or one hundred times the covered diameter of the wire; that is, $5 \mathrm{~cm}$. Since the turns are in contact, the equivalent length may, in this case, also be found by measuring the over-all length of the winding, including the insulation. Both these methods are equivalent to taking one hundred times the pitch of the winding, which, in this case, is equal to the covered diameter of the wire. 
For coil II, the equivaleut radius is $\frac{\mathrm{I}}{2}($ IO.25-O.I $)=5.075 \mathrm{~cm}$. The equivalent length is fifty times $0.2=10 \mathrm{~cm}$.

The dimensions found in this way for coils I and II are to be used in the appropriate current sheet formula. (See p. 73.)

\section{THE MUTUAL INDUCTANCE OF A CIRCLE AND A COAXIAL SINGLE-LAYER COIL}

\section{LORENZ'S FORMULA}

The problem of finding the mutual inductance of a circle and a coaxial single layer winding was first solved by Lorenz. ${ }^{53}$ Assuming that the current was uniformly distributed over the surface of the cylinder, forming a current sheet, he integrated over the length of the cylinder the expression for the mutual inductance of a circular element and the given circle. This expression is an elliptic integral. Lorenz reduced the integrated form to a series and gave the following formula for the mutual inductance of the disk and solenoid of what is now called the Lorenz apparatus. He called it, however, the constant of the apparatus instead of mutual inductance, and denoted it by $C$. It is of course the whole number of lines of magnetic force passing through the disk due to unit current in the surrounding solenoid.

$$
\begin{aligned}
M & =\frac{\pi q r^{2}}{d}\left[Q\left(\alpha_{1}\right)+Q\left(\alpha_{2}\right)\right] \\
Q(\alpha) & =2 \pi q \sqrt{\frac{\alpha-\mathrm{I}}{\alpha}\left[\mathrm{I}+\frac{3}{8} \frac{q^{2}}{\alpha^{2}}+\frac{5}{\mathrm{I} 6} \frac{q^{4}}{\alpha^{4}}\left(\frac{7}{4}-\alpha\right)\right.} \\
& +\frac{35}{\mathrm{I} 28} \frac{q^{6}}{\alpha^{6}}\left(\frac{33}{8}-\frac{9}{2} \alpha+\alpha\right)+\cdots .
\end{aligned}
$$

$\rho=$ radius of the disk, Fig. 25 .

$r=$ radius of the solenoid.

$2 x=$ length of winding of solenoid.

$q=\rho / r=$ ratio of the two radii.

$d=\frac{2 x}{n}=$ distance between centers of successive turns of wire.

$\alpha=\frac{x^{2}+r^{2}}{r^{2}}$

${ }^{53}$ Wied. Annalen, 25, p. I; i885. Oeuvres Scientifiques, 2, p. I62. 
If the disk be not exactly in the mean plane of the solenoid, and $x_{1}$ be the distance from the plane of the disk to one end of the solenoid and $x_{2}$ to the other,

$$
\alpha_{1}=\frac{x_{1}^{2}+r^{2}}{r^{2}} \quad \alpha_{2}=\frac{x_{2}^{2}+r^{2}}{r^{2}}
$$

Then $Q\left(\alpha_{1}\right)$ is found by substituting the values of $\alpha_{1}$ in equation (53) above, and $Q\left(\alpha_{2}\right)$ by using the value of $\alpha_{2}$ for $\alpha$ in the same equation. The sum of these two quantities multiplied by $\frac{\pi q r^{2}}{d}$ gives the constant of the

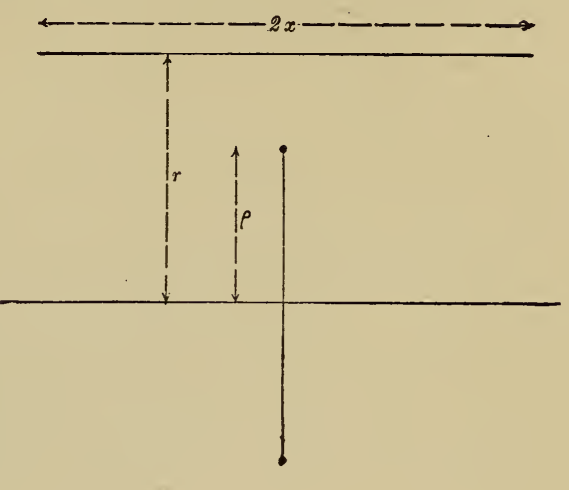
instrument; that is, the mutual inductance sought.

As Lorenz gave the expression Fig: 25 for the general term of (53), his equation can be extended. The following is the general term:

$$
Q(\alpha)=2 \pi \sum_{m=0}^{m=\infty} q^{2 m+1} \frac{\mathrm{I} \cdot 3 \cdots 2 m-\mathrm{I}}{2.4 \cdots 2 m} \cdot \frac{\mathrm{I}}{\mathrm{I} .2 \cdots(m+\mathrm{I})} \cdot \frac{a^{m}}{d a^{m}}\left(\frac{\alpha-\mathrm{I}}{\alpha}\right)^{m+\frac{1}{3}}
$$

\section{JONES'S FORMULAS}

Two solutions of the above problem were given by Jones, ${ }^{54}$ both

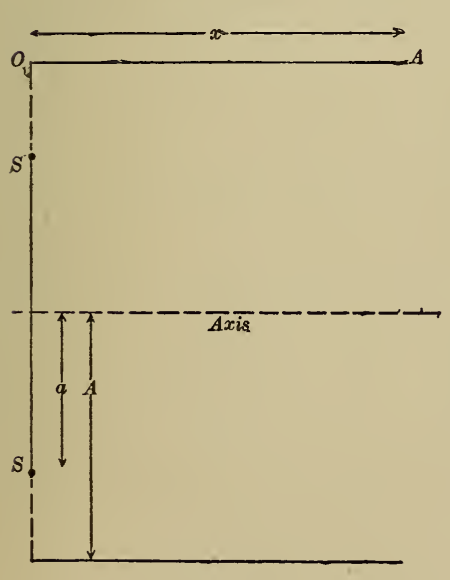

in terms of elliptic integrals. The current was considered to flow not in a current sheet, but along a spiral winding or helix. The first solution was in the form of a series, convergent only when $\mathrm{O}_{1} \mathrm{~A}$, Fig. 26, is less than the difference in the radii of inner and outer coils; that is, when $\mathrm{O}_{1} \mathrm{~A}$ is less than $A-a$. As this is a serious limitation, and the formula is a laborious one to use, it is not here given. The second solution is exact and general, and is in terms of elliptic integrals of all three kinds.

Fig. 26 The second formula is as follows:

$$
M_{\theta}=\Theta(A+a) c k\left\{\frac{F-E}{k^{2}}+\frac{c^{\prime 2}}{c^{2}}(F-\Pi)\right\}
$$


$M_{\theta}=$ mutual inductance of helix $\mathrm{O}_{1} \mathrm{~A}$, Fig. 26, with respect to the disk $\mathrm{S}$ in the plane of one end.

$\Theta=2 \pi n, I / n=$ pitch of winding, $\Theta=$ whole angle of winding. $F, E$, and $\Pi$ are the complete elliptic integrals to modulus $k$, where

$$
k^{2}=\frac{4 A a}{(A+a)^{2}+x^{2}}=\sin ^{2} \gamma, c^{2}=\frac{4 A a}{(A+a)^{2}}, c^{\prime 2}=\mathrm{I}-c^{2} .
$$

$\Pi$, the complete elliptic integral of the third kind, can be expressed in terms of incomplete integrals of the first and second kinds, and the value of $M_{\theta}$ can then be calculated by the help of Legendre's tables. (See example 50.) The calculation is, however, extremely tedious, especially when the value is to be determined with high precision.

Campbell has given Jones's formula (54) a slightly different form, ${ }^{55}$ somewhat more convenient in calculation, as follows:

$$
M=2 \pi n_{1} n_{2}(A+a)\left\{\frac{c}{k}(F-E)+\frac{A-a}{b} \psi\right\}
$$

where $n_{1}$ is the same as $n$ above, the number of turns per $\mathrm{cm}$ on the solenoid, $n_{2}$ is the number of turns in the secondary coil (in the

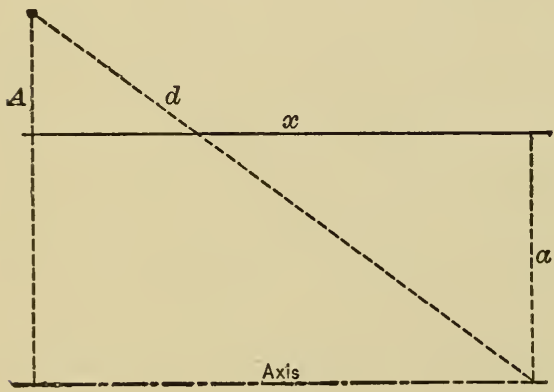

Fig. 27 above case it was taken as one), $A$ is the greater and $a$ the less of the two radii (in the above case $A$ was the radius of the solenoid and $a$ of the circle within), and

$$
\begin{aligned}
& \psi=F(k) E\left(k^{\prime}, \beta\right)- \\
& {[F(k)-E(k)] H\left(k^{\prime}, \beta\right)-\frac{\pi}{2}}
\end{aligned}
$$

where $F(k)$ and $E(k)$ are the complete elliptic integrals to modulus $k$, and $F\left(k^{\prime}, \beta\right)$ and $E\left(k^{\prime}, \beta\right)$ are the incomplete elliptic integrals to modulus $k^{\prime}$ and amplitude $\beta ; k^{\prime}=\cos \gamma, \sin \beta=c^{\prime} \mid k^{\prime} ; k, c$, and $c^{\prime}$ are given above. If a second-

${ }^{55}$ A. Campbell, Proc. Roy. Soc, A, 79, p. 428; 1907. There is a misprint in the formula as given in Campbell's paper. It was, however, used correctly in the numerical calculations given in the paper. 
ary circle or coil has a radius greater than that of the solenoid, the same formula can be used if $A$ is taken for the radius of the larger secondary and $a$ is the radius of the solenoid (Fig. 27).

\section{ROSA'S FORMULA ${ }^{56}$}

The following formula gives the mutual inductance of a single layer coil of length $x$ and a coaxial circle of radius $a$ in the plane of one end of the coil, as shown in Fig. 26. It is the same quantity represented by $M$ of equations (53) and (55) and $M_{\theta}$ of (54).

$$
\begin{array}{r}
M_{\mathrm{OA}}=\frac{2 \pi^{2} a^{2} N}{d}\left[\mathrm{I}+\frac{3}{8} \frac{a^{2} A^{2}}{d^{4}}+\frac{5}{64} \frac{a^{4} A^{4}}{d^{8}} X_{2}+\frac{35}{5 \mathrm{I} 2} \frac{a^{6} A^{6}}{d^{12}} X_{4}+\frac{63}{\mathrm{IO} 24} \frac{a^{8} A^{8}}{d^{16}} X_{6}\right. \\
\left.+\frac{23 \mathrm{I}}{4096} \frac{a^{10} A^{10}}{d^{20}} X_{8}+\frac{429}{8 \mathrm{I} 92} \frac{a^{12} A^{12}}{d^{24}} X_{10}+\cdots\right]
\end{array}
$$

$$
\begin{aligned}
& X_{2}=3-4 \frac{x^{2}}{A^{2}} \\
& X_{4}=\frac{5}{2}-\text { Io } \frac{x^{2}}{A^{2}}+4 \frac{x^{4}}{A^{4}}
\end{aligned}
$$

$X_{6}=\frac{35}{\mathrm{I} 6}-\frac{35}{2} \frac{x^{2}}{A^{2}}+2 \mathrm{I} \frac{x^{4}}{A^{4}}-4 \frac{x^{6}}{A^{6}}$

$X_{8}=\frac{63}{32}-\frac{\text { I05 }}{4} \frac{x^{2}}{A^{2}}+63 \frac{x^{4}}{A^{4}}-36 \frac{x^{6}}{A^{6}}+4 \frac{x^{8}}{A^{8}}$

$X_{10}=\frac{23 \mathrm{I}}{\operatorname{I} 28}-\frac{\operatorname{II} 55}{32} \frac{x^{2}}{A^{2}}+\frac{\operatorname{II} 55}{8} \frac{x^{4}}{A^{4}}-\operatorname{I6} 5 \frac{x^{6}}{A^{6}}+55 \frac{x^{8}}{A^{8}}-4 \frac{x^{10}}{A^{10}}$

(For general coefficient, see p. 63.)

$a=$ radius of disk or circle S, Fig. 26 .

$A=$ radius of the solenoid.

$x=$ length $\mathrm{O}_{1} \mathrm{~A}$ of one end of the solenoid.

$d=\sqrt{x^{2}+A^{2}}=$ half the diagonal of the solenoid.

$N$ is the whole number of turns of wire in the length $x$.

This formula is very easy to use in numerical calculation, notwithstanding it looks somewhat elaborate. The logarithm of $\frac{a^{2} A^{2}}{d^{4}}$, multiplied by $2,3,4$, etc., gives the logarithm of the corresponding factor in each of the other terms. Similarly, the various terms $X_{2}, X_{4}$, etc., contain only powers of $\frac{x^{2}}{A^{2}}$ besides the numerical 
coefficients. It is hence a far simpler matter to compute $M$ with high precision by this formula than by Jones's formula, the latter containing as it does elliptic integrals of all three kinds and involving the tedius interpolations for incomplete elliptic integrals.

If the secondary circle has a larger radius than the solenoid, $A$ will be the radius of the circle and $a$ the radius of solenoid. In every case $A$ is the greater and $a$ the less of the two radii, and $d$ is $\sqrt{A^{2}+x^{2}}$.

Equation (56) may be written

$$
M=\frac{2 \pi^{2} a^{2} n_{1} x}{d} S
$$

where $n_{1}$ is the number of turns of wire per cm, $x$ is the length of the coil, Fig. 26, and $S$ is the value of the quantity in brackets in (56), which is always somewhat greater than unity. This may also be put as follows:

or,

$$
M=a^{2} n_{1}\left(\frac{2 \pi^{2} x}{d}\right) S=a^{2} n_{1} R S
$$

$$
M=a^{2} n_{1} K
$$

The quantity $R$ depends on $x / d$; that is, only upon the shape of the

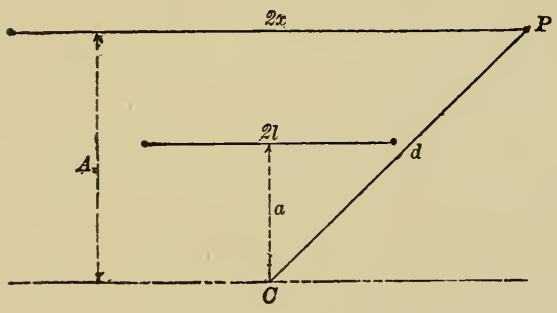

Fig. 28 solenoid. $S$ depends upon $x / A$, $a \mid A$, and $A \mid d$; that is, upon the relative sizes of the inner circle and the solenoid and the shape of the solenoid. If we have the value of $R S$, or $K$ of equation (57) for a given solenoid and circle, we can get $M$ by multiplying by $a^{2} n_{1}$, and for any other system of similar shape but different size by multiplying the same value of $K$ by $a^{2} n_{1}$. The values of the constant $K$ for various values of $\alpha / A$ and $x / A$ are given in Table III, page 193 .

If the disk or circle be in the center of a solenoid of length $2 x$ (Fig. 28), the value of $M$ is of course double that given by using $x$. If it be not quite in the center, the value of $M$ must be calculated for each end separately. 
For illustrations and tests of the above formulas, see examples 48 to $5 \mathrm{I}$, pages IO3-Iro.

\section{EXAMPLES ILLUSTRATING THE FORMULAS FOR THE MUTUAL INDUC- TANCE OF A CIRCLE AND A COAXIAL SOLENOID}

EXAMPLE 48. ROSA'S FORMULA (56) COMPARED WITH JONES'S FORMULA (54)

Professor Jones gave the calculations by formula (54) of the con-. stant of the Lorenz apparatus made for McGill University, obtaining the values given below, the second value being that obtained after the plate had been reground and again measured.

A calculation ${ }^{57}$ of the same two cases by formula (56) gives very closely agreeing results.

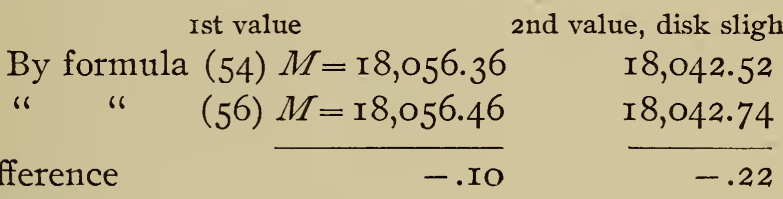

These differences, amounting to five parts in a million in the first case and twelve parts in a million in the second case, are wholly negligible in the most refined experimental work.

EXAMPLE 49. FORMULA (56) COMPARED WITH JONES'S FIRST FORMULA

Take as a second example the case given by Jones ${ }^{58}$ to illustrate his first formula.

$$
\begin{aligned}
& A=\mathrm{ro} \text { inches } \quad a=5 \text { inches } \quad x=2 \text { inches } \\
& d^{2}=\mathrm{I} 04 \frac{a^{2} A^{2}}{d^{4}}=\frac{2500}{\mathrm{I} 08 \mathrm{I} 6} \quad \log \frac{a^{2} A^{2}}{d^{4}}=\overline{\mathrm{I}} \cdot 3638733 \\
& \text { Ist term }=\mathrm{I} .0000000 \\
& X_{2}=2.8400 \\
& X_{4}=2.1064 \\
& X_{6}=\mathrm{I} .5208 \\
& X_{8}=\text { I.OI } 73 \\
& 2 " \text { " }=.086677 \mathrm{I} \\
& X_{10}=0.5^{815} \\
& \frac{2 \pi^{2} a^{2}}{d}=48.38972 \\
& 3 \text { " }=.0118537 \\
& 4 "=.001778 \mathrm{I} \\
& 5 \text { " }=.0002670 \\
& 6 " \text { " }=.0000379 \\
& 7 \text { " }=.0000046
\end{aligned}
$$

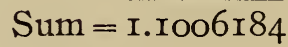

${ }^{57}$ This Bulletin, 3, p. 218; 1907.

${ }^{58}$ Phil. Mag., 27, p. 61; I889. In this example, $P_{0}$ should be 0.654870 instead of 0.54870 , as printed in Jones's article. 
$\therefore M=53.2586$ I $N, N$ being the number of turns of wire on the coil.

Jones gives $M=53.25879 N$.

The difference between these values is three parts in a million.

EXAMPLE 50. CALCULATION OF CONSTANT OF AYRTON-JONES CURRENT BALANCE BY FORMULAS (54) AND (56)

As a further test of the formulas let us calculate the constant of an electro-dynamometer or current balance of the Ayrton-Jones type, ${ }^{58}$ of which AB, Fig. 29, is the upper fixed coil and $E D$ is the moving coil, the circle $S$ at the upper end lying in the plane through the middle of $\mathrm{AB}$ and the circle $\mathrm{R}$ at the lower end of $\mathrm{ED}$ lying in the middle plane of the lower fixed coil $\mathrm{BC}$.
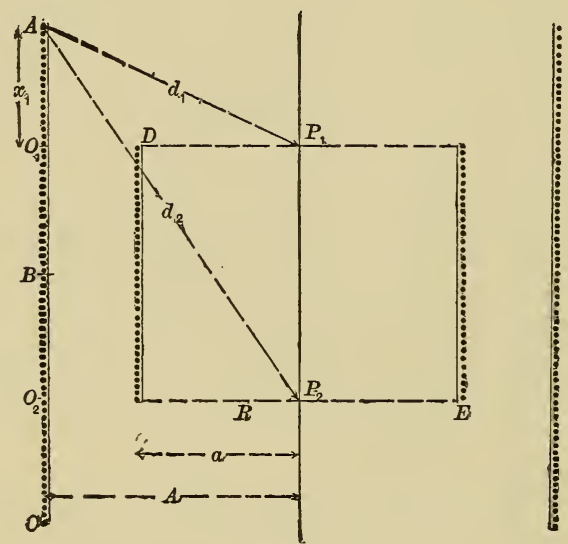

Fig. 29

Assume the dimensions as follows:

$A=16 \mathrm{~cm}=$ radius of fixed coil, Fig. 29.

$a=10 \mathrm{~cm}=$ radius of moving coil.

$x_{1}=8 \mathrm{~cm}=$ half length of $\mathrm{AB}=\mathrm{O}_{1} \mathrm{~A}$

$x_{2}=24 \mathrm{~cm}=\mathrm{I} .5$ times $\mathrm{AB}=\mathrm{O}_{2} \mathrm{~A}$

$n_{1}=$ IO $=$ number of turns per $\mathrm{cm}$

$N_{1}=80 \quad=$ number of turns in distance $\mathrm{O}_{1} \mathrm{~A}=x_{1}$, Fig. 29.

$N_{2}=240=$ number of turns in distance $\mathrm{O}_{2} \mathrm{~A}=x_{2}$ 


$$
\begin{aligned}
& d_{1}=\sqrt{A^{2}+x^{2}}{ }_{1}=8 \sqrt{5}=\text { diagonal } \mathrm{AP}_{1}, \text { Fig. } 29 . \\
& d_{2}=\sqrt{A^{2}+x_{2}^{2}}=8 \sqrt{\mathrm{I}_{3}}=\text { diagonal } \mathrm{AP}_{2}
\end{aligned}
$$

We have to determine two mutual inductances, namely, $M_{\mathrm{S}}$ between the coil $\mathrm{O}_{1} \mathrm{~A}$ of 80 turns on the circle $\mathrm{S}$, and $M_{\mathrm{R}}$ between the coil $\mathrm{O}_{2} \mathrm{~A}$ of 240 turns on the circle $\mathrm{R}$. In each case the circle is in the plane passing through the lower end of the coil.

Formula (56) will be used, taking $N_{1}, x_{1}$, and $d_{1}$ in the first case and $N_{2}, x_{2}$, and $d_{2}$ in the second case.

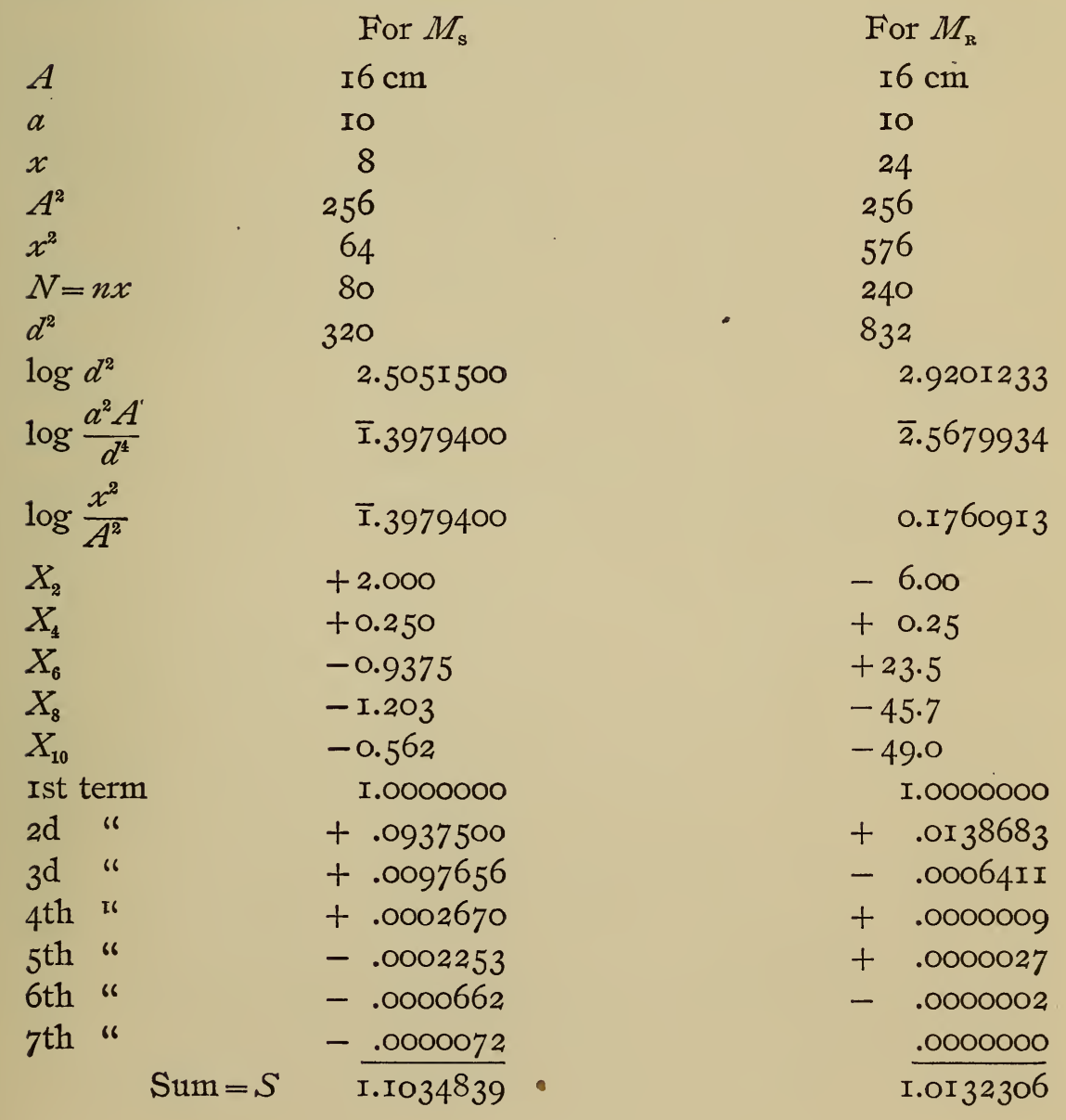




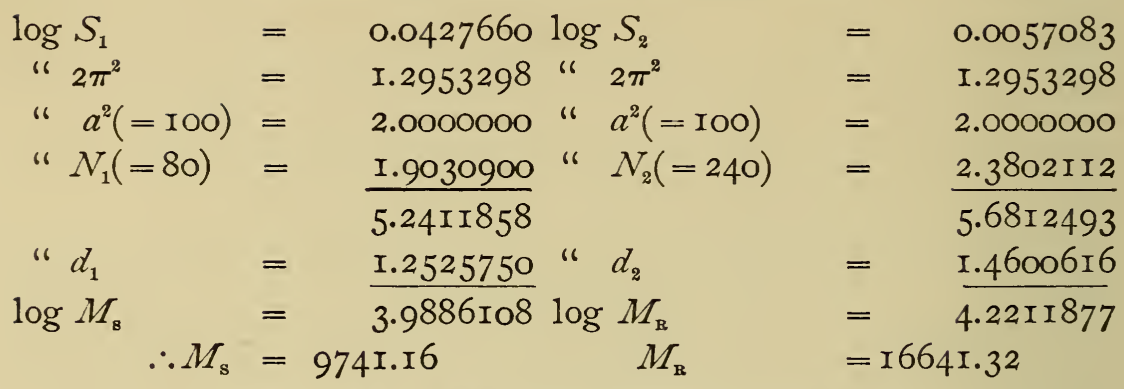

THE SAME EXAMPLE BY JONES'S FORMULA

We will now calculate $M_{\mathrm{S}}$ and $M_{\mathrm{R}}$ by Jones's second formula given above, using also the following equation to find $F-\Pi$ : $\frac{k^{\prime 2} \sin \beta \cos \beta(F-\Pi)}{c}=F(k) E\left(k^{\prime}, \beta\right)+E(k) F\left(k^{\prime}, \beta\right)-F(k) F\left(k^{\prime}, \beta\right)-\frac{\pi}{2}$

$A$

a

$x$

$\Theta=2 \pi N$

$c=\frac{2 \sqrt{A a}}{A+a}$

$c^{\prime}=\sqrt{\mathrm{I}-c^{2}}$

$k=\frac{2 \sqrt{A a}}{\sqrt{(A+a)^{2}+x^{2}}}$

$k^{\prime}=\sqrt{\mathrm{I}-k^{2}}$

$\log \sin \beta\left(\sin \beta=\frac{c^{\prime}}{k^{\prime}}\right)$

$F(k)$

$E(k)$

$\frac{F-E}{k^{2}}$

$F\left(k^{\prime}, \beta\right)$

$E\left(k^{\prime}, \beta\right)$

$\underline{k^{\prime 2} \sin \beta \cos \beta(F-\Pi)}$

$\frac{c^{\prime 2}}{c^{2}}(F-\Pi)$
For $M_{\mathrm{s}}$

I $6 \mathrm{~cm}$

IO

8

I6o $\pi$

0.9730085

0.2307692

0.929981 2

0.3676073

$9.797793^{8}$

2. $437337 \mathrm{I}$

I. 1323456

I. 5088957

0.6852557

o. 672 I 988

$-0.8266738$

-0.685 I 799
For $M_{\mathrm{R}}$ I6 $\mathrm{cm}$

IO

24 $480 \pi$

0.9730085

0.2307692

0.7 I 49701

0.6991 550

9.5186043

I. 863666 I

I. 3449927

I.0146546

0.3394833

o. $33332 \mathrm{OI}$

- I. I 256799

$-0.4045298$ 


$$
\begin{array}{lrr}
\log \left\{\frac{F-E}{k^{2}}+\frac{c^{\prime 2}}{c^{2}}(\mathrm{~F}-\Pi)\right\} & \overline{\mathrm{I}} .9 \mathrm{I} 57773 & \overline{\mathrm{I}} .7854 \mathrm{I} 87 \\
\log (\Theta(A+a) c k) & \frac{4.0728340}{3.9886 \mathrm{II} 3} & \frac{4.4357689}{4.22 \mathrm{II} 876} \\
\log M & M_{\mathrm{S}=974 \mathrm{I} . \mathrm{I} 7 \mathrm{~cm}} & M_{\mathrm{R}}=\mathrm{I} 664 \mathrm{I} .32
\end{array}
$$

$M_{\mathrm{S}}$ differs from the value obtained by formula (I6) by one part in a million, $M_{\mathrm{R}}$ is identical.

$M_{\mathrm{S}}$ is the mutual inductance of the winding $\mathrm{O}_{1} \mathrm{~A}$ on $\mathrm{S}$. The inductance $M_{1}$ of the whole coil $\mathrm{AB}$ on $\mathrm{S}$ is twice as much, that is

$$
M_{1}=\mathrm{I} 9482.34
$$

The inductance of $\mathrm{AB}$ on $\mathrm{R}$ is $M_{\mathrm{R}}$ above, minus the inductance of $\mathrm{O}_{2} \mathrm{~B}$ on $\mathrm{R}$ which is the same as that of $\mathrm{O}_{1} \mathrm{~A}$ on $\mathrm{S}$, that is, $M_{\mathrm{S}}$. Therefore,

$$
M_{2}=\mathrm{I} 664 \mathrm{I} \cdot 32-974 \mathrm{I} \cdot \mathrm{I} 7=6900 . \mathrm{I} 5
$$

Hence $M_{1}-M_{2}=12582.19 \mathrm{~cm}$.

The force of attraction of the one winding $A \cdot B$ in dynes is

$$
\frac{I}{2} f=i_{1} i_{2} n_{2}\left(M_{1}-M_{2}\right) \text {. }
$$

The force due to the second winding BC is equal to this. Suppose $i_{1}=i_{2}=\mathrm{I}$ ampere $=0$.I c.g.s. unit of current and $n_{2}=$ Io turns per $\mathrm{cm}$. Then

$$
\begin{aligned}
& i_{1} i_{2} n_{2}=0 . \text { Io } \\
& \therefore f=0.20 \times 12582 . \text { I } 9 \text { dynes } \\
& =2516.438 \text { dynes } \\
& 2 f=5032.876 \text { dynes }=\text { change of force on reversal of current } \\
& =5.1356 \text { gms where } g=980 .
\end{aligned}
$$

If there are two sets of coils, one on each side of the balance, as in the ampere balance built for the National Physical Laboratory, the force would be doubled again.

In calculating the mutual inductance of the disk and surrounding solenoid in the Lorenz apparatus the series (56) will be more convergent when the winding is long, and of course more convergent when the disk is not of too great diameter. 


\section{EXAMPLE 51. MUTUAL INDUCTANCE OF CAMPBELL'S FORM OF STAND-} ARD BY FORMULAS (55) AND (56)

A cylinder $20 \mathrm{~cm}$ in diameter has two coils of 50 turns each wound as shown in Fig. 30, each covering $5 \mathrm{~cm}(=\mathrm{AB})$ with an interval of Io $\mathrm{cm}$ between $\left(=\mathrm{AA}^{\prime}\right)$. A secondary coil of Iooo turns of finer wire is wound in a channel $\mathrm{S}$, with a mean radius of 14.5 $\mathrm{cm}$. The magnetic field near $\mathrm{S}$, due to the double solenoid, is very weak, and is zero at some point; at this place $M$ will be a maximum, and variations in $M$ due to small changes in $A$ will be very small. To calculate $M$ for the solenoid $\mathrm{AB}$ and the coil $\mathrm{S}$, we take two cases, as in the preceding example. First, $M$ for $\mathrm{S}$ and a winding $\mathrm{O}_{2} \mathrm{~B}$ of roo turns; second, $M$ for $\mathrm{S}$ and $\mathrm{O}_{2} \mathrm{~A}$ of 50 turns. The difference will be $M$ for $\mathrm{S}$ and the actual winding $\mathrm{AB}$. Or, supposing

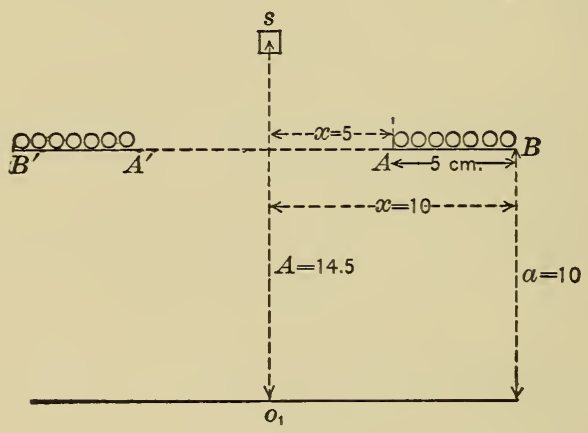

$\overline{0000000}$

$\overline{0000000}$

Fig. 30

$\mathrm{AB}$ to have roo turns, $M$ will be the same as for $\mathrm{AB}$ of 50 and $\mathrm{A}^{\prime} \mathrm{B}^{\prime}$ of 50 . Using formula (55) we have the following values: 


$$
\begin{aligned}
& \text { For } M_{1} \\
& a=\text { IO } \\
& =\mathrm{IO} \\
& A=\mathrm{I} 4.5 \\
& x=b=\text { Iо } \\
& \log k=\quad \mathrm{I} .9590874 \\
& \gamma=65^{\circ} 3 x^{\prime} 7^{\prime \prime} \cdot 32 \\
& k^{\prime}=\sqrt{\text { O.I7I7243 }} \\
& \beta=26^{\circ} I 8^{\prime} 36^{\prime \prime} .85 \\
& \gamma^{\prime}=24^{\circ} 28^{\prime} 52^{\prime \prime} .68 \\
& F=2.326780 \mathrm{I} \\
& E=\mathrm{I} . \mathrm{I} 590043 \\
& \frac{c}{k}(F-E)=\text { I.261 } 3045=\mathbf{1} .6839704 \\
& F\left(k^{\prime}, \beta\right)=0.46 \mathrm{I} 8972=0.756 \mathrm{I} 693 \\
& E\left(k^{\prime}, \beta\right)=0.45653^{14} \\
& \psi=-1.0479404 \\
& \frac{A-a}{b} \psi=-0.47 \text { I } 5732 \\
& \text { For } M_{2} \\
& =\quad \mathrm{I} 4.5 \\
& =5.0 \\
& =\quad 1.98366715 \\
& =74^{\circ} 23^{\prime} 3^{\prime \prime \prime} .88 \\
& =\sqrt{0.07237 \mathrm{II}} \\
& =43^{\circ} 3^{\prime} 33^{\prime \prime} .06 \\
& =\mathrm{I} 5^{\circ} 36^{\prime} 2 \mathrm{I}^{\prime \prime} . \mathrm{I} 2 \\
& =2.73 \mathrm{I} 2000 \\
& =\quad 1.0812388 \\
& =0.7469284 \\
& =-0.7784352 \\
& =-0.7005918 \\
& \frac{c}{k}(F-E)+\frac{A-a}{b} \psi= \\
& 0.78973 \text { I } 3 \\
& =0.9833786 \\
& \begin{array}{rlrl}
n_{1} n_{2} & =200,000 & = & \text { IOO,000 } \\
M_{1}= & 24,3 \mathrm{I} 3,940 \mathrm{~cm} \quad M_{2}= & \text { I } 5, \mathrm{I} 37,940 \mathrm{~cm} \\
=24 \cdot 3 \mathrm{I} 394 \text { millihenrys }= & \text { I } 5 . \mathrm{I} 3794 \text { milli- } \\
\text { henrys }
\end{array} \\
& M=M_{1}-M_{2}=9.1760 \text { millihenrys. }
\end{aligned}
$$

Campbell gives ${ }^{60}$ the value of $M$ as 9.1762 millihenrys, but for want of any particulars of his calculation we do not know wherein the difference lies.

We have worked this problem out also by formula (56) with the following results :

$$
\begin{aligned}
& M_{1}=24 \cdot 3 \text { I } 387 \text { millihenrys } \\
& M_{2}=\text { I5.1 } 3857 \text { " " } \\
& M=9 \cdot \text { I } 7530 ~ " ~
\end{aligned}
$$

The value of $M_{1}$ agrees with that found by (55) to about two parts in a million. $M_{2}$ is, however, a little larger, making $M$ smaller. This is due to the fact that formula $\left(5^{6}\right)$ is not as convergent for

${ }^{60}$ A. Campbell, Proc. Roy. Soc., 79, p. 428; 1907.

$21674^{\circ}-\mathrm{I} 2-8$ 
$x=5$ in this problem as for $x=\mathrm{I}$, and hence the terms neglected after the seventh are appreciable. Hence, for so short a coil as this, formula (54) or (55) will give a more accurate result than (56).

\section{THE SELF-INDUCTANCE OF A CIRCULAR RING OF CIRCULAR SECTION}

\section{KIRCHHOFF'S FORMULA}

The formula for the self-inductance of a circle was first given by Kirchhoff ${ }^{61}$ in the following form:

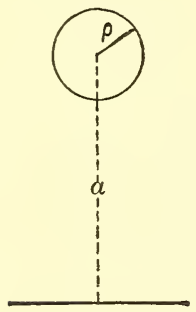

$$
L=2 l\left\{\log \frac{l}{\rho}-\mathrm{r} .508\right\}
$$

where $l$ is the circumference of the circular conductor and $\rho$ is the radius of its cross section. This is equivalent to the following:

$$
L=4 \pi a\left\{\log \frac{8 a}{\rho}-1.75\right\}
$$

a being the radius of the circle, Fig. 31. These formulas are approximate, being more nearly correct as the ratio $\rho / a$ is smaller

\section{MAXWELL'S FORMULA}

Fig. 31

A more accurate expression, obtained by means of Maxwell's principle of the geometrical mean distance, is the following:

$$
L=4 \pi a\left\{\left(\mathrm{I}+\frac{3}{\mathrm{I} 6} \frac{R^{2}}{a^{2}}\right) \log \frac{8 a}{R}-\left(2+\frac{R^{2}}{\mathrm{I} 6 a^{2}}\right)\right\}
$$

Substituting in this equation the value of the geometrical mean distance for a circular area, $R=\rho e^{-\frac{1}{4}}=.7788 \rho$, we obtain ${ }^{62}$

$$
L=4 \pi a\left\{\left(\mathrm{I}+\text { O.II } 37 \frac{\rho^{2}}{a^{2}}\right) \log \frac{8 a}{\rho}-.0095 \frac{\rho^{2}}{a^{2}}-\text { I.75 }\right\}
$$

This is a very accurate formula for circles in which the radius of section $\rho$ is very small in comparison with the radius $a$ of the circle. The geometrical mean distance $R$ has, however, been computed on the supposition of a linear conductor, and can only 
be applied to circles of relatively small value of $\rho / a$, and the square of the geometrical mean distance is used for the arithmetical mean square distance in the second order terms. We must therefore expect an appreciable error in formula (6I) when the ratio $\rho / \alpha$ is not very small. Formulas (58), (59), and (6r) have been deduced on the supposition of a uniform distribution of the current over the cross section of the ring.

If the ring is a hollow, circular, thin tube, or if the current in the ring is alternating and of extremely high frequency, so that it can be regarded as flowing on the surface of the ring, the geometrical mean distance for the section would be the radius $\rho$, and we should have instead of $(6 \mathrm{I})$ the following by substituting $R=\rho$,

$$
L=4 \pi a\left\{\left(\mathrm{I}+\frac{3}{\mathrm{I} 6} \frac{\rho^{2}}{a^{2}}\right) \log \frac{8 a}{\rho}-\underset{\mathrm{I} 6 a^{2}}{\rho^{2}}-2\right\}
$$

In the case of solid rings carrying alternating currents of moderate frequency the value of $L$ would be somewhere between the values given by (6I) and (62).

\section{RAYLEIGH AND NIVEN'S FORMULA}

Rayleigh and Niven gave, ${ }^{63}$ without proof, the following formula for a circular coil of $n$ turns and of circular section, ${ }^{64}$ which is more nearly exact than either of the preceding:

$$
L=4 \pi n^{2} a\left\{\left(\mathrm{I}+\frac{\rho^{2}}{8 a^{2}}\right) \log \frac{8 a}{\rho}+\frac{\rho^{2}}{24 a^{2}}-\mathrm{I} .75\right\}
$$

When $n=\mathrm{I}$, this will be the self-inductance of a single circular ring. ${ }^{65}$ This formula neglects higher powers of $\frac{\rho}{a}$ than the second,

${ }^{63}$ Rayleigh's Collected Papers, Vol. II, p. I5.

${ }^{64}$ Neglecting the correction for effect of insulation and shape of section of the separate wires.

${ }^{65}$ Max Wien, Wied. Annalen, 53, p. 928, 1894, derived by direct integration of Maxwell's formula (I2) over the cross section of the ring, the formula

$$
I=4 \pi a\left\{\left(\mathrm{I}+\frac{\rho^{2}}{8 a^{2}}\right) \log \frac{8 a}{\rho}-0.008_{3} \frac{\rho^{2}}{a^{2}}-\mathrm{I} .75\right\}
$$

It was shown, however, by Terezawa, Tokyo Math. Phys. Soc., 5, p. 84, 1909, that this formula is in error, the correct result being identical with that of Rayleigh and Niven (63). This result was verified by Mr. Cohen at the Bureau of Standards in I909, and quite recently independently by Mr. T. J. Bromwich of Cambridge, England. The error of Wien's expression is in practical cases of no importance. 
and its error therefore depends on the magnitude of the ratio of the radius of the cross section to the radius of the ring. Assuming, as is probably justified, that the coefficients of the terms in $\left(\frac{\rho}{a}\right)^{4}$, are of the same magnitude, or smaller, than those of the terms in $\left(\frac{\rho}{a}\right)^{2}$, the error will not be greater than $\frac{\mathrm{I}}{\text { IOOOOO }}$ even for $\frac{\rho}{a}=$ o.I, an exceptionally unfavorable case.

If used for a coil of more than one turn, the expression for $L$ must be corrected for the space occupied by the insulation between the wires and for the shape of the section. ${ }^{66}$

\section{SELF-INDUCTANCE OF A TUBE BENT INTO A CIRCLE}

Suppose that the cross section of the ring is not solid, but is an annulus bounded by two concentric circles of radii $\rho_{1}$ and $\rho_{2}, \rho_{2}$ being the larger. Then assuming the current to be uniformly distributed over the cross section, we find ${ }^{67}$ by means of Wien's method

$$
\begin{aligned}
L=4 \pi a & {\left[\left(\mathrm{I}+\frac{\rho_{1}{ }^{2}+\rho_{2}{ }^{2}}{8 a^{2}}\right) \log \frac{8 a}{\rho_{2}}-\mathrm{I} \cdot 75+\frac{2 \rho_{2}{ }^{2}+\rho_{1}{ }^{2}}{32 a^{2}}\right.} \\
& -\frac{\rho_{1}{ }^{2}}{2\left(\rho_{2}{ }^{2}-\rho_{1}{ }^{2}\right)}+\frac{\rho_{1}{ }^{4}}{\left(\rho_{2}{ }^{2}-\rho_{1}{ }^{2}\right)^{2}}\left(\mathrm{I}+\frac{\rho_{1}{ }^{2}}{8 a^{2}}\right) \log \frac{\rho_{2}}{\rho_{1}} \\
& \left.-\frac{\rho_{2}{ }^{4}+\rho_{1}{ }^{2} \rho_{2}{ }^{2}+\rho_{2}{ }^{4}}{48 a^{2}\left(\rho_{2}{ }^{2}-\rho_{1}{ }^{2}\right)}\right]
\end{aligned}
$$

In this formula terms of higher order than $\frac{\rho_{2}{ }^{2}}{a^{2}}$ and $\frac{\rho_{1}{ }^{2}}{a^{2}}$ have been neglected. Expanding (64) in terms of $\frac{\rho_{2}{ }^{2}-\rho_{1}{ }^{2}}{a^{2}}$ and letting $\rho_{1}$ approach $\rho_{2}$ we find for the case of a tube with infinitely thin walls, or of a tube carrying a current of infinitely high frequency,

$$
L=4 \pi a\left[\left(\mathrm{I}+\frac{\rho^{2}}{4 a^{2}}\right) \log \frac{8 a}{\rho}-2\right]
$$

${ }^{66}$ See Rosa, this Bulletin, 3, p. I; I907.

${ }^{67}$ Grover, Phys. Rev., 30, p. 787; rgro. 
a result which was also found by direct integration, ${ }^{68}$ and which was subsequently communicated to us by Mr. T. J. Bromwich.

This corresponds to Maxwell's equation (62), but as might be expected gives a slightly greater value for the inductance.

If we expand (64) in terms of $\frac{\rho_{1}}{\rho_{2}}$ and let $\rho_{1}$ approach zero, we find for the limiting case of a ring with a solid cross section, the same formula $(63)$ as was derived by directly performing the integration for this case.

An important case is that of a ring of solid cross section, where the current is not distributed uniformly over the cross section, but the current density is proportional to the distance from the axis of the ring. This would apply to the case of a ring revolving about a diameter in a uniform magnetic field. For this Wien (loc. cit.) derived the formula

$$
L=4 \pi a\left\{\left(\mathrm{I}+\frac{3}{8} \frac{\rho^{2}}{a^{2}}\right) \log \frac{8 a}{\rho}-.092 \frac{\rho^{2}}{a^{2}}-\mathrm{I} .75\right\}
$$

\section{J. J. THOMSON'S FORMULA FOR RING OF ELLIPTICAL SECTION}

If the circular ring has an elliptical section the approximate formula for its self-inductance (corresponding to (59) for a circular section) is ${ }^{69}$

$$
L=4 \pi a\left\{\log \frac{\mathrm{I} 6 a}{\alpha+\beta}-\mathrm{I} .75\right\}
$$

where $\alpha$ and $\beta$ are the semiaxes of the ellipse, and $\alpha$ is the mean radius of the circular ring.

The formulas of Minchin, ${ }^{70}$ Hicks,${ }^{71}$ and Bláthy ${ }^{72}$ we have elsewhere ${ }^{73}$ shown to be incorrect, and hence they are not here given.

${ }^{68}$ Russell also gives equation (65) but without the term in $\frac{\rho^{2}}{a^{2}}$ in Phil. Mag., 13, p. $430 ; 1907$.

${ }^{69}$ J. J. Thomson, Phil. Mag., 23, p. 384 ; 1886.

${ }^{70}$ Phil. Mag., 37, p. 300; I894.

${ }^{71}$ Phil. Mag.. 38, p. 456; I894.

${ }^{72}$ London Electrician, 24, p. 630; Apr. 25, I89o.

${ }^{73}$ This Bulletin, 4, p. I49; I907. 


\section{EXAMPLES ILLUSTRATING THE FORMULAS FOR THE SELF-INDUC-} TANCE OF CIRCULAR RINGS OF CIRCULAR SECTION

\section{EXAMPLE 52. COMPARISON OF FOUR FORMULAS FOR THE SELF-INDUC- TANCE OF CIRCLES}

For a circle of radius $\alpha=25 \mathrm{~cm}$ and $\rho=0.05 \mathrm{~cm}$ we obtain from the four formulas the following values of $L$ :

By Kirchhoff's formula (59)

By Maxwell's formula (6I)

By Rayleigh and Niven's (63)

By Wien's second formula (66)

$$
\begin{aligned}
& L=654.40496 \pi \mathrm{cm} \\
& L=654.40533 \pi \mathrm{cm} \\
& L=654.40548 \pi \mathrm{cm} \\
& L=654.406 \mathrm{I} 7 \pi \mathrm{cm} .
\end{aligned}
$$

Thus for so small a value of $\frac{\rho}{a}$ as $1 / 500$ any of these formulas is sufficiently accurate, the greatest difference being less than one in a million, except in the case of formula (66).

EXAMPLE 53. SECOND COMPARISON OF FOUR FORMULAS FOR CIRCLES

For a circle of radius $a=25 \mathrm{~cm}, \rho=0.5 \mathrm{~cm}, \frac{\rho}{a}$ being $\mathrm{I} / 50$.

By Kirchhoff's formula (59)

By Maxwell's formula (6I)

By Rayleigh and Niven's formula $\left(6_{3}\right)$

By Wien's second formula (66)

$$
\begin{aligned}
& L=424 \cdot 1464 \pi \mathrm{cm} \\
& L=424 \cdot 1734 \pi \mathrm{cm} \\
& L=424 \cdot 178 \mathrm{I} \pi \mathrm{cm} \\
& L=424.2326 \pi \mathrm{cm} .
\end{aligned}
$$

EXAMPLE 54. THIRD COMPARISON OF FOUR FORMULAS FOR CIRCLES

For a circle of radius $a=\mathrm{IO} \mathrm{cm}, \rho=\mathrm{I} .0, \frac{\rho}{\alpha}=\mathrm{I} / \mathrm{IO}$.

By Kirchhoff's formula (59)

By Maxwell's formula (6I)

By Rayleigh and Niven's formula (63)

By Wien's second formula (66)

$$
\begin{aligned}
& L=105.28 \mathrm{I} \pi \mathrm{cm} \\
& L=105.476 \pi \mathrm{cm} \\
& L=105.517 \pi \mathrm{cm} \\
& L=105.902 \pi \mathrm{cm} .
\end{aligned}
$$

It will be seen that for the smallest ring of radius $10 \mathrm{~cm}$ and diameter of section $2 \mathrm{~cm}$ Maxwell's formula gives a result I part in 2500 too small, while the simple approximate formula of Kirchhoff is in error by one in four hundred. For the larger rings the differences are much smaller.

Wien's second formula gives appreciably larger values than the others, as it should do. 
EXAMPLE 55. COMPARISON OF FORMULAS (62) AND (65) FOR VERY THIN WALLED TUBES

(a) $a=25 \quad \rho=0.05 \mathrm{~cm}$

By Maxwell's formula (62)

$L=629.40556 \pi \mathrm{cm}$

By Formula (65)

Solid ring $\left(6_{3}\right)$

$L=629.40579 \pi \mathrm{cm}$

$L=654.40548 \pi \mathrm{cm}$

(b) $a=25 \quad \rho=0.5 \mathrm{~cm}$

By Maxwell's formula (62)

$L=399.1889 \pi \mathrm{cm}$

By Formula (65)

Solid ring $(63)$

$L=399.2064 \pi \mathrm{cm}$

$L=424.178 \mathrm{I} \pi \mathrm{cm}$

(c) $a=$ IO $\quad \rho=1.0 \mathrm{~cm}$

By Maxwell's formula (62)

$L=95.585 \pi \mathrm{cm}$

By Formula (65)

Solid ring (63)

$L=95.719 \pi \mathrm{cm}$

$L=105.517 \pi \mathrm{cm}$.

Maxwell's expression is nearly correct for the larger ring, but the error increases rapidly as the ratio $\frac{\rho}{a}$ is increased.

EXAMPLE 56. FORMULA (64) FOR A TUBULAR RING

$a=20 \quad \rho_{2}=0.5 \mathrm{~cm}=$ external radius of the cross section.

The calculation has been carried through for different thicknesses of the walls of the tube $\left(\rho_{2}-\rho_{1}\right)$ ranging from zero (infinitely thinwalled tube) to $\rho_{2}$ (solid cross section).

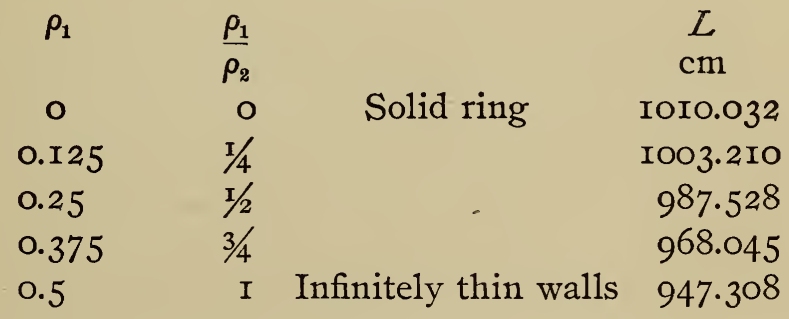

In formula (64), next to the first two terms, the fourth and fifth terms are the most important. 


\section{THE SELF-INDUCTANCE OF A SINGLE LAYER COIL OR SOLENOID}

The following approximate formula for the self-inductance of a long solenoid is often given:

$$
L=4 \pi^{2} a^{2} n_{1}^{2} b
$$

where $a$ is the mean radius, $n_{1}$ is the number of turns of wire per $\mathrm{cm}$, and $b$ is the length, supposed great in comparison with $a$. There is a considerable error in this formula, due to the end effect, but the variations in $L$ due to changes in $l$ are almost exactly proportional to the changes in $l$, and hence this formula may be used for calculating the corresponding variations in $L$.

\section{RAYLEIGH AND NIVEN'S FORMULAS}

The following formula ${ }^{74}$ for the self-inductance of a single layer winding on a solenoid is very accurate when the length $b$ is small compared with the radius $a$, Fig. 32 :

$$
L_{s}=4 \pi a n^{2}\left\{\log \frac{8 a}{b}-\frac{1}{2}+\frac{b^{2}}{32 a^{2}}\left(\log \frac{8 a}{b}+\frac{1}{4}\right)\right\}
$$

$n$ is the whole number of turns of wire on the coil, and the radius is measured to the center of the wire. The length $b$ is the mean

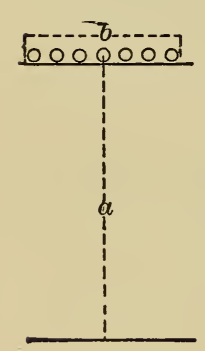

Fig. 32 over-all length including the insulation on the first and last wires if the coil is wound closely with insulated wire. (See also p. 97.)

The self-inductance $L_{s}$ is, however, not the actual self-inductance of the coil, but the current sheet value; that is, it is the value of the self-inductance if the winding were of infinitely thin tape, so that the current would cover the entire length $b$. To get the actual self-inductance $L$ for any given case one must correct $L_{s}$ by formula (8o) below. The same remark applies to all the formulas in this section for $L_{8}$. The approximate formula (68) is too rough to make it worth while to apply such a correction.

For a coil in which the axial dimension $b$ is zero and the radial depth is $c$, the following current sheet formula of Rayleigh and Niven gives the self-inductance:

${ }^{74}$ Proc. Roy. Soc., 32, pp. I04-I4I; I88I. Rayleigh's Collected Papers, 2, p. 15. 


$$
L_{s}=4 \pi a n^{2}\left\{\log \frac{8 a}{c}-\frac{1}{2}+\frac{c^{2}}{96 a^{2}}\left(\log \frac{8 a}{c}+\frac{43}{12}\right)\right\}
$$

This is not an important case in practice.

Formulas (69) and (70) may be obtained from (88) by making first $c=0$ and then $b=0$.

\section{COFFIN'S FORMULA}

Coffin ${ }^{75}$ has extended formula (69) so that it is very accurate for coils of length as great as the radius, and sufficiently accurate for most purposes for coils considerably longer than this.

$$
\begin{gathered}
L_{s}=4 \pi a n^{2}\left\{\log \frac{8 a}{b}-\frac{\mathrm{I}}{2}+\frac{b^{2}}{32 a^{2}}\left(\log \frac{8 a}{b}+\frac{\mathrm{I}}{4}\right)-\frac{\mathrm{I}}{\operatorname{IO} 24} \frac{b^{4}}{a^{4}}\left(\log \frac{8 a}{b}-\frac{2}{3}\right)\right. \\
\left.+\frac{\mathrm{IO}}{\mathrm{I} 3 \mathrm{IO} 72} \frac{b^{6}}{a^{6}}\left(\log \frac{8 a}{b}-\frac{\operatorname{IO9}}{\mathrm{I} 20}\right)-\frac{35}{4 \mathrm{I} 94304} \frac{b^{8}}{a^{8}}\left(\log \frac{8 a}{b}-\frac{43 \mathrm{I}}{420}\right)\right\} \quad[7 \mathrm{I}] \\
\text { LORENZ'S FORMULA }
\end{gathered}
$$

\section{LORENZ'S FORMULA}

Lorenz first gave ${ }^{76}$ an exact formula for the self-inductance of a

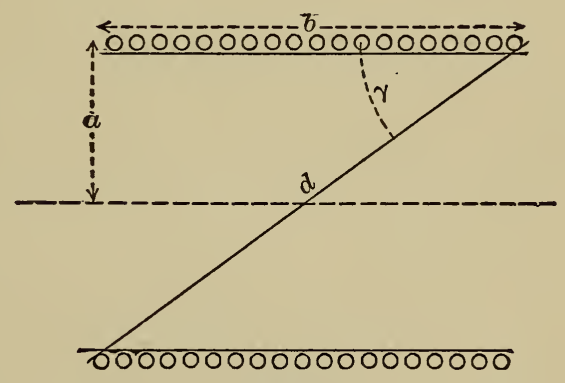

Fig. 33

single layer solenoid. It is, like the others, a current sheet formula, and requires correction by (8o) for a winding of wire, but applies to a solenoid of any length. Changing the notation slightly Lorenz's formula as originally given is as follows:

${ }^{75}$ This Bulletin, 2, p. II 3 ; 1906.

${ }^{76}$ Wied. Anna1., 7, p. I6I; 1879. Oeuvres_Scientifiques de L. Lorenz, Tome, 2, I, p. Ig6. 


$$
L_{s}=\frac{32}{3} \frac{\pi n^{2} a^{3}}{b^{2}}\left\{\frac{2 k^{2}-\mathrm{I}}{k^{3}} \cdot E+\frac{\mathrm{I}-k^{2}}{k^{3}} F-\mathrm{I}\right\}
$$

where $k^{2}=\frac{4 a^{2}}{4 a^{2}+b^{2}}$ and $F$ and $E$ are complete elliptic integrals of the first and second kind of modulus $k$, and $a, b$, and $n$ are the radius (Fig. 33), length, and whole number of turns of wire, respectively. By simple substitutions the formula may be put into the following form, where $d$ is the diagonal of the solenoid $=\sqrt{4 a^{2}+b^{2}}$;

$$
L_{s}=\frac{4 \pi n^{2}}{3 b^{2}}\left\{d\left(4 a^{2}-b^{2}\right) E+d b^{2} F-8 a^{3}\right\}
$$

Coffin derived ${ }^{77}$ an expression for $L$ in elliptic integrals which is equivalent to (73), and also obtained (73) from an expression ${ }^{78}$ attributed to Kirchhoff.

Formula (73) may be written

or

$$
L_{s}=a n^{2}\left[\frac{8 \pi}{3}\left\{\sqrt{\mathrm{I}+\frac{b^{2}}{4 a^{2}}}\left(\frac{4 a^{2}}{b^{2}}-\mathrm{I}\right) E+\sqrt{\mathrm{I}+\frac{b^{2}}{4 a^{2}}} F-\frac{4 a^{2}}{b^{2}}\right\}\right]
$$

where $a$ is the radius of the solenoid, $n$ is the whole number of turns on the coil, and $Q$ is the function of $\frac{2 a}{b}(=\tan \gamma)$ contained in the square brackets. We have calculated $Q$ for various values of $\tan \gamma$ from 0.2 to 4.0 and given them in Table IV, page I94. This table will be found useful in calculating $L_{s}$ for solenoids when $\tan \gamma$ has one of the values given in the table, as all calculation of elliptic integrals is avoided. In problems where the length and diameter can be chosen at will, as in the designing of apparatus, this method of calculating $L$ will be most frequently useful. The values of the constant $Q$ given in the table have been computed with great care, so that they give very accurate values of $L_{s}$, for long as well as short solenoids.

In calculating the value of $L_{s}$ by means of formula (69), (7I), (73), or (74) and the following, one should use for the length $b$ the over-all

77 This Bulletin, 2, p. I23, equation (3I); 1906.

${ }^{78}$ This Bulletin, 2, p. I27, equation (36). The notation is slightly different. 
length including the insulation (A B, Fig. 34, and not $a b$ ) for a close winding of insulated wire, or $n$ times the pitch for a uniform winding of bare or covered wire, which is, of course, the same as the length from center to center of $n+I$ turns. The radius $a$ is the mean radius to the center of the wire. The same method of taking the breadth and depth $b$ and $c$ applies in the formulas of section 7 . (See also remarks under example 47.)

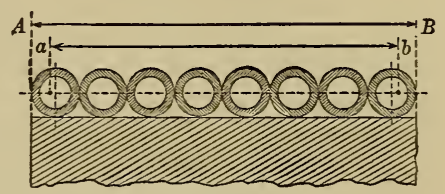

Fig. 34

\section{NAGAOKA'S FORMULAS AND TABLES}

In a recent paper ${ }^{79}$ Nagaoka has derived formulas and prepared tables by which the self-inductance of a cylindrical current sheet of any dimensions whatever may be accurately and conveniently calculated. Starting from his absolute formula (45) for the mutual inductance of coaxial solenoids, he passes to the special case that the two solenoids coincide, and shows that the resulting expression for the self-inductance is equivalent to Lorenz's absolute formula (73), which he then expands in terms of $q$ or $q_{1}$ functions.

He expresses the inductance of a coil of finite length by means of the expression (68) for an infinitely long coil, introducing a correction factor $K$, which is less than unity, to take account of the effect of the ends of the coil.

Thus

$$
L=4 \pi^{2} a^{2} n_{1}^{2} b K=4 \pi^{2} a^{2} \frac{n^{2}}{b} K
$$

where $K$ is a function of half the angular aperture $\theta$ of the coil at the center. Nagaoka has prepared tables giving $K$ with $\theta$ as argument and also as function of the $\frac{\text { diameter }}{\text { length }}=\frac{2 a}{b}$. These tables are reproduced here as Tables XX and XXI, and enable $K$ to be obtained by interpolation with all the accuracy that will usually be required. In case, however, it becomes necessary to obtain a more accurate value of $K$ than can be obtained from these tables, or in such cases as fall outside the range of the tables, or in a portion where the function is changing so rapidly as to make interpolation difficult, the following formulas may be used to calculate $K$ directly.

${ }^{79}$ Jour. Coll. Sci. Tokyo, 27, art. 6, pp. 18-33; r909. 
For short solenoids

$$
K=\frac{\mathrm{I}}{3 \pi \sqrt{q_{1}\left(\mathrm{I}+\alpha_{1}\right)^{2}}}\left[\mathrm{I}-\frac{k^{\prime 2}}{k^{2}}+\left\{\frac{k^{\prime 2}}{k^{2}}\left(\mathrm{I}+\frac{8 \beta_{1}}{\mathrm{I}+\alpha_{1}}\right)+\frac{8 \gamma_{1}}{\mathrm{I}-\delta_{1}}\right\} \frac{\mathrm{I}}{2} \log _{e} \frac{\mathrm{I}}{q_{1}}\right]-\frac{4}{3 \pi} \frac{k}{k^{\prime}}
$$

where

$$
\begin{array}{ll}
\alpha_{1}=q_{1}^{2}+q_{1}{ }^{6}+q_{1}^{12}+\ldots & \\
\beta_{1}=q_{1}^{2}+3 q_{1}^{6}+6 q_{1}^{12}+\cdots & k^{2}=\frac{4 a^{2}}{4 a^{2}+b^{2}} \\
\gamma_{1}=q_{1}-4 q_{1}^{4}+9 q_{1}^{9}-\ldots & k^{2}=\frac{b^{2}}{4 a^{2}+b^{2}} \\
\delta_{1}=2 q_{1}-2 q_{1}^{4}+2 q_{1}^{9}-\ldots & \\
q_{1}=\frac{l_{1}}{2}+2\left(\frac{l_{1}}{2}\right)^{5}+\mathrm{I} 5\left(\frac{l_{1}}{2}\right)^{9}+\ldots . & \\
l_{1}=\frac{\mathrm{I}-\sqrt{k}}{\mathrm{I}+\sqrt{k}}=\frac{k^{\prime 2}}{(\mathrm{I}+k)(\mathrm{I}+\sqrt{k})^{2}} &
\end{array}
$$

For relatively long coils

$$
K=\frac{2}{3(\mathrm{I}-\delta)^{2}}\left\{\mathrm{I}+\frac{8 \beta}{\mathrm{I}+\alpha}+\frac{k^{\prime 2}}{k^{2}} \cdot \frac{8 \gamma}{\mathrm{I}-\delta}\right\}-\frac{4}{3 \pi} \frac{k}{k^{\prime}}
$$

where $k$ and $k^{\prime}$ have the same values as in (76) and

$$
\begin{aligned}
& q=\frac{l}{2}+2\left(\frac{l}{2}\right)^{5}+\mathrm{I} 5\left(\frac{l}{2}\right)^{9}+\ldots \\
& l=\frac{\mathrm{I}-\sqrt{k^{\prime}}}{\mathrm{I}+\sqrt{k^{\prime}}}=\frac{k^{2}}{\left(\mathrm{I}+k^{\prime}\right)\left(\mathrm{I}+\sqrt{k^{\prime}}\right)^{2}}
\end{aligned}
$$

and $\alpha, \beta, \gamma, \delta$ are given by the same equations as $\alpha_{1}, \beta_{1}, \gamma_{1}, \delta_{1}$ in $(76)$ substituting $q$ in place of $q_{1}$. Table XV will be found convenient in obtaining $q$ and $q_{1}$ from $\frac{l}{2}$ and $\frac{l_{1}}{2}$. The more complicated expressions for the latter are to be used only when it becomes difficult to obtain $\mathrm{I}-\sqrt{k^{\prime}}$ and $\mathrm{I}-\sqrt{k}$ without carrying out the calculation of $k$ and $k^{\prime}$ to an inconvenient number of decimal places.

For relatively long coils, for which the angle $\theta=\tan ^{-1} \frac{2 a}{b}$ is not greater than $45^{\circ}$, the simple formula 


$$
\begin{aligned}
K=\mathrm{I} & -\frac{4}{3 \pi} \frac{k}{k^{\prime}}+2 q+\mathrm{I} 2 q^{2}+44 q^{3}+\mathrm{Ir} 6 q^{4}+260 q^{5} \\
& +576 q^{6}+\frac{3760}{3} q^{7}+\cdots
\end{aligned}
$$

will give values of $K$ correct to a few parts in ten million in the most unfavorable case.

The formulas (76), (77), and (78) between them cover the entire range of values of $\theta$ with all the precision desired, since the general terms of the series are known. The formula (76) for short coils is the least convenient to use, and for very short coils (69) is preferable. However, by including terms in $q^{9}$ in (77) the range of its applicability may be extended to $\theta=80^{\circ}$, so that $(76)$ need not be used except as a check.

\section{THE WEBSTER-HAVELOCK FORMULA}

Webster ${ }^{80}$ in 1905 by the evaluation of a definite integral, involving Bessel functions, derived a formula for the inductance of relatively long solenoids, which is very simple in form. Havelock ${ }^{81}$ gives the same formula as a special application of his formulas for the values of certain integrals of Bessel functions, and stated that the first four terms had already been found by Russell, ${ }^{82}$ but seems to have been unacquainted with the work of Webster. This formula is

$$
\begin{gathered}
L=4 \pi^{2} \frac{a^{2} n^{2}}{b}\left\{\mathrm{I}-\frac{8}{3 \pi} \frac{a}{b}+\frac{\mathrm{I}}{2} \frac{a^{2}}{b^{2}}-\frac{\mathrm{I}}{4} \frac{a^{4}}{b^{4}}+\frac{5}{\mathrm{I} 6} \frac{a^{6}}{b^{6}}\right. \\
\left.-\frac{35}{64} \frac{a^{8}}{b^{8}}+\frac{\mathrm{I} 47}{\mathrm{I} 28} \frac{a^{10}}{b^{10}}-\cdots \cdot\right\}
\end{gathered}
$$

Both Webster and Havelock gave the same expression for the general term of this series, viz:

$$
\frac{(-\mathrm{I})^{s}(2 s) !(2 s+2) !}{s !(s+2) !\{(s+\mathrm{I}) !\}^{2} 2^{2 s+1}}\left(\frac{a}{b}\right)^{2 s+2}
$$

${ }^{80}$ Bull. of Amer. Math. Soc., 14, No. I, p. I; 1907.

${ }^{81}$ Phil. Mag., 15, p. 332; I908.

${ }^{82}$ Phil. Mag., 13, eq. (48), p. 445 ; I907. 
but in all the terms of Webster's final equation (20), except the first two, a factor 2 has been omitted in the denominator of the coefficients.

This expression (79) is in the form adopted by Nagaoka, the expression in the brackets being equivalent to the correction for the ends $K$ tabulated by Nagaoka.

\section{ROSA'S CORRECTION FORMULA}

Rosa has shown ${ }^{83}$ that the above formulas (69 to 79) apply accurately only to a winding of infinitely thin strip which completely covers the solenoid (the successive turns being supposed to meet at the edges without making electrical contact) and so realizing the uniform distribution of current over the cylindrical surface which has been assumed in the derivation of all the formulas. A winding of insulated wire or of bare wire in a screw thread may have a greater or less self-inductance than that given by the current sheet formulas above according to the ratio of the diameter of the wire to the pitch of the winding. Putting $L$ for the actual self-inductance of a winding and $L_{s}$ for the current sheet value given by one of the above formulas,

$$
L=L_{s}-\Delta L
$$

The correction $\Delta L$ is given by the following expression:

$$
\Delta L=4 \operatorname{\pi an}[A+B]
$$

where as above $a$ is the radius, $n$ the whole number of turns of wire and $A$ and $B$ are constants given in Tables VII and VIII, pages I 97 and 199.

The correction term $A$ depends on the size of the (bare) wire (of diameter $d$ ) as compared with the pitch $D$ of the winding; that is, on the value of the ratio $d / D$. For values of $d / D$ less than $0.58, A$ is negative, and in such cases when the numerical values of $A$ are greater than the value of $B$, which is always positive, the correction $\Delta L$ will be negative, and hence $L$ will be greater than $L_{s}$. (See examples $5^{8}$ and 63 .)

${ }^{83}$ This Bulletin, 2, pp. I6I-I87; 1906. 


\section{THE SUMMATION FORMULA FOR $\mathrm{L}^{84}$}

If we have a single layer winding on a cylinder (Fig. 35), the selfinductance is equal to the sum of the self-inductances of the separate turns plus the sum of the mutual inductances of each wire on all the others. Thus, if there are $n$ turns

$$
L=n L_{1}+2(n-\mathrm{I}) M_{12}+2(n-2) M_{13}+2(n-3) M_{14}+\cdots+2 M_{1 n} \quad[8 \mathrm{I}]
$$

where $L_{1}$ is the self-inductance of a single turn, $M_{12}$ is the mutual inductance of the first and second turns or any two adjacent turns, $M_{13}$ is the mutual inductance of the first and third or of any two turns separated by one, etc., and $M_{1 n}$ is the mutual inductance of the first and last turns. For a coil of four turns this becomes

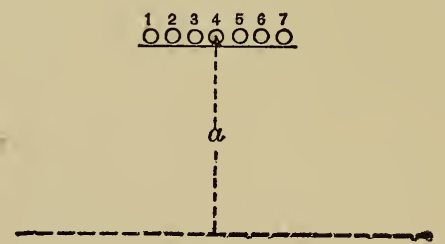

Fig. 35

$$
L=4 L_{1}+6 M_{12}+4 M_{13}+2 M_{14}
$$

$L_{1}$ should be calculated by formula $\left(6_{3}\right)$ or any formula for a circular ring and $M_{12}$, etc., by (12) or (13). When the number of turns on the coil is small, formula (8I) is very convenient, and gives very accurate results.

\section{STRASSER'S FORMULA}

Strasser ${ }^{85}$ has derived a formula for the self-inductance of a single layer coil of few turns from (8I) by substituting for $L_{1}$ its value as given by formula (59) and for the various $M$ 's their values as given by (I2). Strasser's formula with slight correction and some changes in notation is as shown on next page $:^{86}$

${ }^{84}$ Kirchhoff, Gesammelte Abhandlungen, p. I77.

85 Wied. Annal., 17, p. 763 ; 1905.

${ }^{86}$ Strasser uses the formula for $L$ as: $L=4 \pi a\left(\log \frac{a}{\rho}+0.333\right)$. This is not quite correct. It should be

$$
L_{1}=4 \pi a\left(\log \frac{8 a}{\rho}-\mathrm{r} .75\right)=4 \pi a \log \left(\frac{a}{\rho}-\mathrm{r} .75+\log _{e} 8\right)=4 \pi a\left(\log \frac{a}{\rho}+0.32944\right) .
$$




$$
\begin{aligned}
L=4 \pi a & {\left[n\left(\log \frac{8 a}{\rho}-\mathrm{I} \cdot 75\right)+n(n-\mathrm{I})\left(\log \frac{8 a}{d}-2\right)-A\right.} \\
+ & \left.\left.\frac{d^{2}}{8 a^{2}}\left(3 \log \frac{8 a}{d}-\mathrm{I}\right)\left(\frac{n^{2}\left(n^{2}-\mathrm{I}\right)}{\mathrm{I} 2}\right)-B\right\}\right]
\end{aligned}
$$

where $n$ is the whole number of turns, $d$ is the pitch, or distance between the centers of two adjacent turns, $a$ is the mean radius of the coil, $\rho$ is the radius of the section of the wire, and $A$ and $B$ are constants given by Table V, page I95, for values of $n$ up to 30 . For coils of a larger number of turns (or indeed any number of turns) the value of $L$ can be accurately calculated by (90) and (93) or by (73) and (80).

\section{SELF-INDUCTANCE OF TOROIDAL COIL OF RECTANGULAR SECTION}

The first approximation to the self-inductance of a toroidal coil (that is, a circular solenoid) of rectangular section, wound with a single layer of $n$ turns of wire is

$$
L_{s}=2 n^{2} h \log \frac{r_{2}}{r_{1}}
$$

where $h$ is the axial depth of the coil, and $r_{1}$ and $r_{2}$ are the inner and outer radii of the ring, Fig. 36 . Formula $\left(8_{3}\right)$ is exact for a toroidal

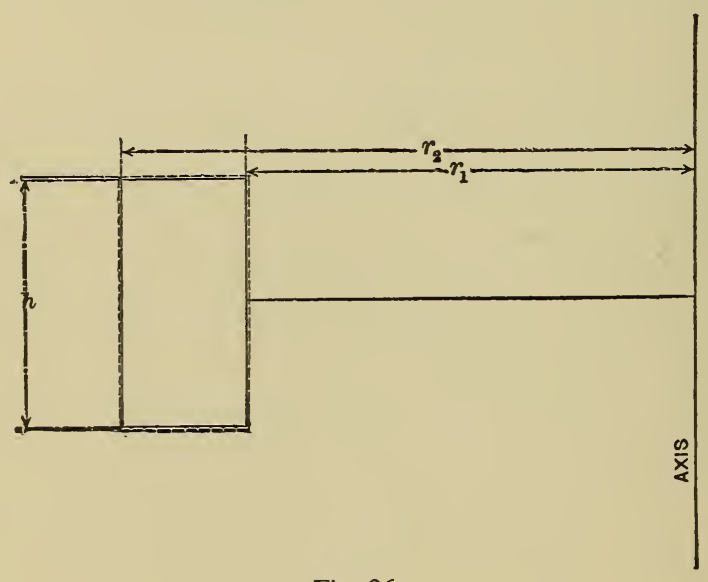

Fig. 36

core enveloped by a current sheet, or for a winding of $n$ turns of infinitely thin tape covering the core completely, the core within 
the current sheet being $h \mathrm{~cm}$ in axial height and $\left(r_{2}-r_{1}\right) \mathrm{cm}$ in radial breadth.

When the core is wound with round insulated wire, the selfinductance is affected by those lines of force within the cross section of the wire itself, and by those linked with each separate turn of wire in addition to those running through the core. Rosa has shown $^{87}$ that the total self-inductance may be more or less than the current sheet value given by $(83)$ according to the size of the wire and the pitch of the winding. In every case, however, the correct value of the self-inductance is derived from the current sheet value $L_{s}$ by subtracting a correction term $\Delta L$, which is equal to twice the length of the wire multiplied by the sum of two quantities $A$ and $B$. Thus

$$
L=L_{s}-2 n l(A+B)
$$

where $n$ is the whole number of turns in the winding, $l$ is the length of one turn, $A$ is a quantity, depending on the diameter of the wire and the pitch of the winding, given in Table VII, and $B$ is 0.332. When $A$ is negative and greater than $B, L$ is greater than $L_{s}$. This occurs when the pitch of the winding is more than 2.5 times the diameter of the (uncovered) wire.

Fröhlich's formula ${ }^{88}$ based on the assumption that a winding of round wires is equivalent to a thick current sheet has been shown to be incorrect. ${ }^{89}$

\section{CHOICE OF FORMULAS}

For a coil of only a few turns the summation formula (8I), or Strasser's formula (82) give the inductance with great accuracy without the necessity of correction by Tables VII or VIII. Strasser's formula is, however, accurate only for short solenoids, so that the pitch of the winding can not be very great.

For very short solenoids Rayleigll and Niven's formula (69) will give values correct to one in ten thousand for coils whose axial length is as great as one-quarter the diameter of the coil; Coffin's

${ }^{87}$ This Bulletin, 4, p. I4I; 1907.

${ }^{88}$ Wied. Annal., 63, p. I42; 1897.

${ }^{89}$ This Bulletin, 4, p. I4I; 1907.

$2 \times 674^{\circ}-12 \longrightarrow 9$ 
extension of this expression ( $7 \mathrm{I}$ ) gives as great an accuracy for coils as long as one-half the diameter. These two formulas are probably the most convenient for very short solenoids.

For solenoids longer than about one-fifth their diameter the inductance may perhaps most readily be calculated by Nagaoka's formula (75), and the Tables XX and XXI. Havelock's formula (79) is accurate and convenient for coils whose axial length is greater than about one and a quarter times the diameter.

For purposes of great precision, formulas (76), (77), and (78) may be used, $(76)$ being indicated for coils shorter than about one-fifth the diameter, (77) for coils longer than this, and (78) for coils longer than the diameter. Lorenz's absolute formula (73) is of course applicable to coils of all lengths. The interpolation of the elliptic integrals is, however, most easily carried out for coils whose length ranges between one-fifth of the diameter and equality with the latter. The form of this formula is such as to make it necessary in some cases to calculate the separate terms to a greater number of places than are required in the result.

It must be remembered that all these formulas, with the exception of Strasser's and the summation formula (8I) give values for a current sheet, and must be corrected to reduce to the actual winding of round wires. This requires the use of formula (8o) and Tables VII and VIII.

EXAMPLES ILLUSTRATING THE FORMULAS FOR THE INDUCTANCE OF SINGLE LAYER SOLENOIDS

EXAMPLE 57. RAYLEIGH AND NIVEN'S FORMULA (69) AND CORRECTION FORMULA (80) COMPARED WITH THE SUMMATION FORMULA (81)

$a=25 \mathrm{~cm}, b=\mathrm{I} \mathrm{cm}, n=10$ turns Fig. 37. Suppose the bare wire is $0.8 \mathrm{~mm}$ diameter, the covered wire $\mathrm{r} .0 \mathrm{~mm}$.

By formula (69)

$$
\begin{aligned}
L_{s} & =4 \pi \times 25 \times 100\left\{\log _{e} 200-\frac{\mathrm{I}}{2}+\frac{\mathrm{I}}{20,000}\left(\log _{e} 200+\frac{\mathrm{I}}{4}\right)\right\} \\
& =10,000 \pi \times 4.798595 \\
& =47,985.95 \pi \mathrm{cm}
\end{aligned}
$$

which is the value of $L$ for a current sheet. 
The correction $\Delta L$ by formula (8o) is $\Delta L=\mathrm{IOOO} \pi(A+B)$

Since $D=\mathrm{I} .0 \mathrm{~mm}$ and $d=0.8 \mathrm{~mm}, d / D=0.8$

$$
\begin{aligned}
\text { By Table VII, } A & =0.3337 \\
\text { "VIII, } B & =0.2664 \\
A+B & =\overline{0.6001} \\
\therefore \quad \Delta L & =600 . \mathrm{I} \pi \mathrm{cm} .
\end{aligned}
$$

The value of $\Delta L$ calculated to one place more of decimals is $\Delta L=600.16 \pi \mathrm{cm}$

$$
\begin{array}{rlrl} 
& \therefore \quad L & L & =47985.95 \pi-600.16 \pi \\
\text { or, } & & L & =47385.79 \pi \mathrm{cm} .
\end{array}
$$

The value of $L$ may also be calculated by the summation formula (8I), using Rayleigh and Niven's formula (63) for $L_{1}$ and Maxwell's formula (12), for the $M$ 's. The following are the values of the ten terms of (8I) and the resulting value of $L$ :

$$
\begin{aligned}
\text { Io } L_{1} & =6767.196 \pi \mathrm{cm} \\
\text { I } 8 M_{12} & =10081.664 \pi \\
\text { I6 } M_{13} & =7852.535 \pi \\
\text { I } 4 M_{14} & =6303.439 \pi \\
\text { I } 2 M_{15} & =5057.868 \pi \\
\text { Io } M_{16} & =3991.888 \pi \\
8 M_{17} & =3047.787 \pi \\
6 M_{18} & =2193.465 \pi \\
4 M_{19} & =1408.982 \pi \\
2 M_{110} & =680.982 \pi \\
\text { Sum }=L & =47385.806 \pi \mathrm{cm} .
\end{aligned}
$$

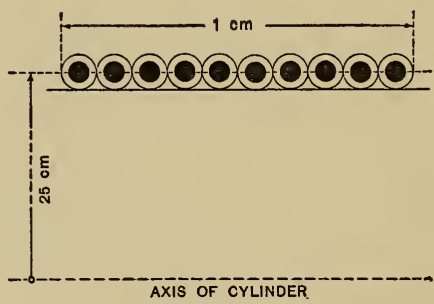

Fig. 37

The difference of less than one in a million between the results obtained by formulas (69) and (8o) combined and formula (8I) is a good check on the corrections of (80), which amount in this case to more than I per cent of the value of the self-inductance. Formula (69) for as short a coil as this is very accurate, the next term, the fourth term of (7r), being inappreciable.

If we attempt to use Lorenz's formula in the above example we notice, first, that $\gamma$ is nearly $89^{\circ}$. The elliptic integrals must consequently be calculated by the series formulas (3), which give their value with all the accuracy desired. We meet, however, with the difficulty that the first and third terms are very nearly equal to one 
another and are several hundred times as large as the second term and the sum of the three terms. Consequently, using seven-place logarithms, it is impossible to obtain the self-inductance closer than about five parts in one hundred thousand.

This is also an unfavorable case for (76). Using seven-place logarithms we find

$$
K=2 \mathrm{I} .28 \mathrm{I} 755-2 \mathrm{I} .220657=0.06 \mathrm{IO} 98
$$

and consequently

$$
L_{s}=47986.27 \pi
$$

which is about one part in one hundred thousand larger than the correct value.

\section{EXAMPLE 58}

As an extreme case to test the use of formulas (69) and (80) we may calculate the self-inductance of a single turn of wire. Let us take the particular case already calculated by Maxwell's and Rayleigh and Niven's formulas (6I) and (63), example 52. The radius $a=25 \mathrm{~cm}$, the diameter of the bare wire $=\mathrm{I} \mathrm{mm}$. We may now assume that the wire is covered and that the diameter $D$ is $2 \mathrm{~mm}$. Then $\frac{d}{D}=0.5$. In using Rayleigh's current sheet formula we take the length of the equivalent current sheet as equal to $D$. We thus have

$$
\begin{aligned}
L_{s} & =4 \pi a\left\{\log _{e} \frac{200}{0.2}-\frac{\mathrm{I}}{2}+\frac{0.04}{20000}\left(\log _{e} \frac{200}{0.2}+\frac{\mathrm{I}}{4}\right)\right\} \\
& =\mathrm{I} 00 \pi\left\{6.907755-0.5+\frac{7.16}{500000}\right\} \\
& =640.777 \pi \mathrm{cm} .
\end{aligned}
$$

From Tables VII and VIII $A=-0.1363$ and $B=0$. Carrying the value of $A$ to one place of decimals more the value is $A=-0.13628$. Thus, since $n=\mathrm{I}, \Delta L=4 \pi a(-0.13628)=-\mathrm{I} 3.628 \pi$, and being negative is added to $L_{s}$. Hence

$$
\begin{aligned}
L & =(640.777+\mathrm{I} 3.628) \pi \\
& =654.405 \pi .
\end{aligned}
$$


This is identical with the value given by the other formulas, example 52 .

If we had taken the bare wire of diameter O.I $\mathrm{cm}$ as equivalent to a current sheet $0.1 \mathrm{~cm}$ long in the above formulas for $L_{s}$, we should have obtained a different value for $L_{s}$, but in that case $\frac{d}{D}$ would be unity and $A$ would be +.5568 . The resulting value of $L$ would, however, be the same as above.

EXAMPLE 59. COFFIN'S FORMULA (71) COMPARED WITH LORENZ'S (73)

We will use for this case a single layer coil wound on an accurately measured marble cylinder belonging to the Bureau of Standards.

Length of winding, $l=30.5510 \mathrm{~cm}=b$ in formula $(73)$

Radius " " $a=27.0862 \mathrm{~cm}$

Number of turns $n=440$

By $(7 x)$

$$
\begin{aligned}
L_{\varepsilon}= & 4 \pi \overline{440}^{2} \times 27.0862\{\mathrm{r} .4590686+0.087824 \mathrm{I}-0.0020427 \\
& +.000165 \mathrm{I}-0.0000204\} \\
= & 4 \pi \overline{440}^{2} \times 27.0862 \times 1.5449947 \\
= & \text { I0I } 80999 \mathrm{~cm}=0.10180999 \text { henry. }
\end{aligned}
$$

By (73)

$$
\begin{aligned}
d^{2}=4 a^{2}+b^{2} & =3868.0128 \\
4 a^{2}-b^{2} & =200 \text { I. } 285^{8} \\
\gamma & =60^{\circ} 34^{\prime} 43 .^{\prime \prime} 655 \\
\log F & =0.3369388 \\
\text { " } E & =0.08 \mathrm{II}_{33}
\end{aligned}
$$

Then

$$
L_{s}=\frac{4 \pi \cdot \overline{440}^{2}}{3(30 \cdot 55)^{2}} \cdot\{\mathrm{I} 50050.12+\mathrm{r} 26105 \cdot 36-\mathrm{I} 58977.00\}
$$

or, $L_{s}=$ IOI810100 $\mathrm{cm}=0$. IoI 8 roro henry. 
The correction to be applied to these values is as follows, the diameter of the bare wire being $0.0634 \mathrm{~cm}$, and consequently $\frac{d}{D}$ $=0.9135$ :

$$
\begin{aligned}
A & =0.4664 \\
B & =0.3353 \\
\hline(A+B) & =0.8017 \\
4 \pi n a & =108.3448 \times 440 \pi=4767 \mathrm{I} .7 \pi \\
\therefore \Delta L & =\mathrm{I} 20067 \mathrm{~cm}
\end{aligned}
$$

and

$$
\begin{aligned}
& L=0.10168992 \text { henry by Coffin's formula } \\
& L=0.10169003 \text { " " by Lorenz's formula. }
\end{aligned}
$$

The agreement between these two formulas is very satisfactory, although in Coffin's formula $b$ is greater than $a$. For shorter coils the accuracy of this formula is better; for longer coils the error rapidly increases.

EXAMPLE 60. NAGAOKA'S FORMULAS (75) AND (77)

We will take for this the coil in the preceding example

$$
a=27.0862 \quad b=30.55 \text { I0 } \quad n=440
$$

Here $\frac{2 a}{b}=\mathrm{I} .773 \mathrm{I} 8$ and by interpolation in Table XXI using third differences we find

$$
\begin{aligned}
K & =0.557885-.003{ }_{16} 6-.000023-.00000 \mathrm{I} \\
& =0.554696
\end{aligned}
$$

For this case $\theta=60^{\circ} 34^{\prime} 43 \cdot \cdot^{\prime \prime} 655=60^{\circ} \cdot 57879$, which gives, by interpolation in Table $\mathrm{XX}$,

$$
\begin{aligned}
K & =0.560382-.0057 \mathrm{I} 2+.000027-.00000 \mathrm{I} \\
& =0.554696
\end{aligned}
$$

Substituting this value of $K$ in (75) we find

$$
L_{s}=\text { O. IоI8IOI } 3
$$

which differs only three parts in ten million from the value found by Lorenz's formula.

Calculating $K$ by (77) we find

$$
\begin{aligned}
\sqrt{k^{\prime}} & =0.70087516 \\
\frac{l}{2} & =0.087932623
\end{aligned}
$$




$$
\begin{aligned}
& q=0.087943 \mathrm{I} 42^{2} \\
& q^{2}=0.007733997 \\
& q^{4}=5.9815 \times \mathrm{IO}^{-5} \\
& q^{6}=4.626 \times \mathrm{Io}^{-7}
\end{aligned}
$$

$$
\begin{array}{rlrl}
\therefore \alpha & =0.00773446 & \gamma & =0.087703884 \\
\beta & =0.007735385 & \mathrm{I}-\delta & =0.82423335
\end{array}
$$

$$
\begin{aligned}
\frac{8 \gamma}{(\mathrm{I}-\delta)} \cdot \frac{k^{\prime 2}}{k^{2}} & =0.27074040 \\
\mathrm{I}+\frac{8 \beta}{\mathrm{I}+\alpha} & =\frac{\mathrm{I} .06 \mathrm{I} 40809}{\mathrm{Sum}}=\overline{\mathrm{I} .332 \mathrm{I} 4849}
\end{aligned}
$$

multiplied by

$$
\begin{aligned}
\frac{2}{3(\mathrm{I}-\delta)^{2}} & =\mathrm{I} .3072568 \\
\frac{4}{3 \pi} \frac{k}{k^{\prime}} & =0.75^{2} 5609 \\
\therefore K & =0.5546959
\end{aligned}
$$

If we make the calculation with formula $(76)$

$$
\begin{aligned}
& \mathrm{I}+\sqrt{k}=\mathrm{I} .93329106 \\
& \mathrm{I}+k=\mathrm{I} .87 \mathrm{IO} 32 \mathrm{IO} \\
& \log _{10} k^{\prime 2}=\overline{\mathrm{I}} \cdot 3825629 \\
& \therefore \frac{l_{1}}{2}=\frac{k^{\prime 2}}{(\mathrm{I}+k)(\mathrm{I}+\sqrt{\bar{k}})^{2}}=0.017252700 \\
& q_{1}=0.017252703 \\
& q_{1}^{2}=0.0002976555 \\
& q_{1}^{4}=8.86 \times \mathrm{ro}^{-8} \\
& \log _{10} \frac{I}{q_{1}}=\mathrm{I} .7631429 \\
& \therefore \frac{\mathrm{I}}{2} \log _{e} \frac{\mathrm{I}}{q_{1}}=2.0298933 \\
& \mathrm{I}+\alpha_{1}=\mathrm{I} .00029766 \\
& \mathrm{I}+\frac{8 \beta_{1}}{\mathrm{I}+\alpha_{1}}=\mathrm{I} .00238054 \\
& \begin{aligned}
\frac{k^{\prime 2}}{k^{2}}\left(\mathrm{I}+\frac{8 \beta_{1}}{\mathrm{I}+\alpha_{1}}\right) & =0.3 \mathrm{I} 88065^{8} \\
\frac{8 \gamma_{1}}{\mathrm{I}-\delta_{1}} & =\frac{0 . \mathrm{I} 4295 \mathrm{I} 27}{\mathrm{Sum}}=0.46 \mathrm{I} 75785 \\
\times \frac{\mathrm{I}}{2} \log _{e} \frac{\mathrm{I}}{q_{1}} & =0.9373 \mathrm{I} 902
\end{aligned}
\end{aligned}
$$




$$
\mathrm{I}-\frac{k^{\prime 2}}{k^{2}}=\frac{0.6819505^{8}}{\mathrm{Sum}}=\mathrm{I.61926960}
$$

multiplied by $\frac{\mathrm{I}}{3 \pi \sqrt{q_{1}\left(\mathrm{I}+\alpha_{1}\right)^{2}}}=\mathrm{I} \cdot 3072565$

$$
\begin{aligned}
& \frac{4}{3 \pi} \frac{k}{k^{\prime}}=0.7525609 \\
& \therefore K=0.5546956
\end{aligned}
$$

The two formulas give the same value of $K$ within about one part in two million.

The corresponding values of $L_{8}$ are:

$$
\begin{aligned}
& L_{s}=0.1018 \text { I010 by }(77) \\
& L_{s}=0.1018 \text { 1005 “ }(76)
\end{aligned}
$$

the former value being identical with that found by Lorenz's formula. This example illustrates well the advantage of obtaining $K$ from Tables XX and XXI rather than by calculation. The accuracy of these tables is ordinarily more than sufficient.

The correction to be applied to these current sheet values $L_{s}$ to obtain the self-inductance $L$, is the same as that calculated in the preceding example.

F.XAMPLE 61. WEBSTER-HAVELOCK FORMULA (79) COMPARED WITH NAGAOKA'S FORMULA (78). LONG COIL

$$
a=\text { Io } \quad b=40 \quad N=400
$$

and suppose the diameter of the bare wire to be $0.05 \mathrm{~cm}$

$$
\begin{aligned}
I+\frac{\mathrm{I} a^{2}}{2 b^{2}} & =\text { I.03I } 25000 \\
-\frac{\mathrm{I}}{4} \frac{a^{4}}{b^{4}} & =-0.00097656 \\
\frac{5}{\mathrm{I} 6} \frac{a^{6}}{b^{6}} & =0.00007629 \\
-\frac{35}{64} \frac{a^{8}}{b^{8}} & =-0.00000834 \\
\frac{\mathrm{I} 47}{\mathrm{I} 28} \frac{a^{10}}{b^{10}} & =0.00000110 \\
-\frac{693}{512} \frac{a^{12}}{b^{12}} & =-0.00000008 \\
\text { Sum } & =1.0303424 \mathrm{I}
\end{aligned}
$$




$$
\begin{aligned}
-\frac{8}{3 \pi} \frac{a}{b} & =-\frac{0.2 \mathrm{I} 220657}{K}=0.8 \mathrm{1} 8 \mathrm{1} 35^{8} 4
\end{aligned}
$$

which gives

By (78)

$$
L_{s}=\text { o.oI } 2919483 \text { henry }
$$

$$
\begin{aligned}
& k^{2}=\frac{\mathrm{I}}{5} \quad k^{\prime 2}=\frac{4}{5} \quad \sqrt{k^{\prime}}=0.94574 \mathrm{I} 52 \\
& \mathrm{I}+k^{\prime}=\mathrm{I} .894427 \mathrm{I} 4 \\
& \therefore \frac{l}{2}=\frac{\mathrm{I}}{2\left(\mathrm{I}+\sqrt{k^{\prime}}\right)^{2}\left(\mathrm{I}+k^{\prime}\right)}=0.013942859 \\
& q=0.013942860 \\
& \mathrm{I}+2 q=\mathrm{I} .02788572 \\
& \text { I } 2 q^{2}=0.00233284 \\
& 44 q^{3}=0.00011926 \\
& \text { I I } 6 q^{4}=0.00000438 \\
& 260 q^{5}=0.00000014 \\
& 576 q^{6}=0.00000000 \\
& \text { Sum }=\mathrm{I} .03034^{2} 34 \\
& \frac{4}{3 \pi} \frac{k}{k^{\prime}}=0.21220657 \\
& K=0.8 \text { I } 8 \text { I } 3577 \\
& \therefore L_{s}=0.012919482 \text { henry }
\end{aligned}
$$

which differs by only one part in ten million from the value by the Webster-Havelock formula. The value of $K$ found by interpolation in Nagaoka's tables is $K=0.818136$.

If we solve this problem by means of Lorenz's formula we are met by the difficulty that $\gamma=26^{\circ}$, and therefore the integrals $F$ and $E$ must be taken from Table XII where their values can not be found more accurately than one part in a million.

We find

$$
\begin{aligned}
d\left(4 a^{2}-b^{2}\right) E & =-87909.94 \\
d b^{2} F & =\text { I I } 8752.95 \\
-8 a^{3}= & -\quad 8000.00 \\
\text { Sum } & =\frac{30843.01}{2} \\
\therefore L_{s} & =\text { 0.01291949 henry. }
\end{aligned}
$$


To find the correction to the current sheet value we have $\frac{d}{D}=0.5$, $n=400$

$$
\begin{aligned}
A & =-0.1363 \\
B & =+0.335 \mathrm{I} \\
A+B= & 0.1988 \\
4 \pi n a(A+B) & =9999 \mathrm{~cm} \\
& =0.0000 \text { IOoo henry, }
\end{aligned}
$$

which must be subtracted from the values of $L_{s}$ to obtain the selfinductance.

EXAMPLE 62. STRASSER'S FORMULA (82) COMPARED WITH (69) AND (80) AND WITH (81)

Take the coil of ro turns used in example 57

$\begin{aligned} a=25, & d=0.10 \quad \rho=0.04, \quad n= \\ \text { From Table V, } & A=97.9226 \quad B=424 \mathrm{I} .59\end{aligned}$

$$
a=25, \quad d=\text { 0. I0 } \quad \rho=0.04, \quad n=\text { Io. }
$$

Substituting in (82),

$$
\begin{aligned}
L=\mathrm{IOO} \pi & {\left[\mathrm{IO}\left(\log _{e} \frac{200}{.04}-\mathrm{I} .75\right)+90\left(\log _{e} \frac{200}{\mathrm{O} . \mathrm{I}}-2\right)-97.9226\right.} \\
& \left.+\frac{\mathrm{O.OI}}{5 \mathrm{OOO}}\left\{\left(3 \log _{e} \frac{2 \mathrm{OO}}{\mathrm{O} . \mathrm{I}}-\mathrm{I}\right) \frac{9900}{\mathrm{I} 2}-424 \mathrm{I} .59\right\}\right]
\end{aligned}
$$

or, $L=100 \pi[473.8306+0.0275]=47385.8 \mathrm{r} \pi \mathrm{cm}$.

This very close agreement with the results by the other two methods (see example 57) is a confirmation of the accuracy of the constants $A$ and $B$ of Table $\mathrm{V}$. Of course, a close agreement with (8I) is to be expected, for (82) is derived directly from (8I).

EXAMPLE 63. FORMULAS (83) AND (84) FOR TOROIDAL COILS

Professor Fröhlich's standard of self-inductance had the following dimensions:

$r_{2}=35.05377 \mathrm{~cm}=$ outer mean radius.

$r_{1}=24.97478 \mathrm{~cm}=$ inner mean radius.

$h=20.08455 \mathrm{~cm}=$ height, center to center of wire.

$\rho=0.0$ I I $47 \mathrm{~cm}=$ radius of wire.

$n=273^{8} \quad=$ whole number of turns. 
These values substituted in $(83)$ give

$$
L_{s}=0.1020893 \text { henry. }
$$

The correction $\Delta L=-2 n l(A+B)$ to be substituted in (84) to give the true value of $L$ is found as follows:

The mean spacing of the winding is $D=\pi \frac{r_{1}+r_{2}}{n}=0.0689$

The diameter of the bare wire $\quad d=2 \rho \quad=0.0223$

From Table VII,

$$
\therefore d / D=0.324
$$

$$
\begin{aligned}
A & =-0.572 \\
B & =+0.33^{20} \\
\therefore A+B & =-0.240
\end{aligned}
$$

$2 n l=2 \times 2738 \times 60.327=330300 \mathrm{~cm}=$ whole length of wire in winding.

$$
\begin{aligned}
-2 n l(A+B) & =+79,300 \mathrm{~cm} \\
& =0.0000793 \text { henry } \\
L_{s} & =0.1020893 \\
L & =0.1021686
\end{aligned}
$$

Thus, the correction increases the value of the self-inductance. If the insulation were thinner and the wire thicker (with the same pitch) the correction might be of opposite sign. Thus, if $\rho$ were 0.02 and hence $d / D$ were $0.58, A$ would be +0.012 and $\Delta L$ would then be 0.000 I I 30 and $L=0.1019763$ henry, considerably less than the preceding value.

\section{THE SELF-INDUCTANCE OF A CIRCULAR COIL OF RECTANGULAR SECTION}

\section{MAXWELL'S APPROXIMATE FORMULA}

Maxwell first gave ${ }^{91}$ an approximate formula for the important case of a circular coil or conductor of rectangular section, Fig. 38, as follows:

$$
L=4 \pi a n^{2}\left(\log \frac{8 a}{R}-2\right)
$$

\footnotetext{
${ }_{90}$ This Bulletin, 4, p. I4I; I907. This value applies to any toroidal coils, of 24 turns or more.

${ }^{91}$ Elect. and Mag., Vol. II, \& 706.
} 
where $R$ is the geometrical mean distance of the cross section of the coil or conductor. The current is supposed uniformly distributed over this section.

The value of $R$ for any given shape of rectangular section is given by (I 24). Its value for several particular cases is

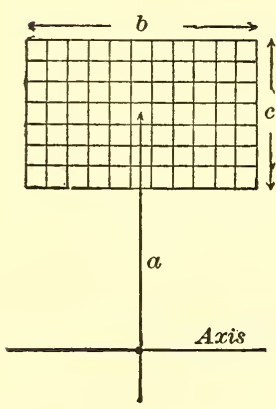

Fig. 38 given in the table on page $I 68$. It is very nearly proportional to the perimeter of the rectangle and approximately equal to $0.2235(\alpha+\beta)$ where $\alpha$ and $\beta$ are the length and breadth of the rectangle.

Formula (85) is derived from (I I) by putting $R$, the geometrical mean distance of the area of the section of the coil from itself, in place of $r$, the distance between two circles. If we use (I2) instead of (II) for this purpose, we shall have a closer approximation to the value of $L$. Thus,

$$
L=4 \pi a n^{2}\left\{\log \frac{8 a}{R} \cdot\left(\mathrm{I}+\frac{3 R^{2}}{\mathrm{I} 6 a^{2}}\right)-\left(2+\frac{R^{2}}{\mathrm{I} 6 a^{2}}\right)\right\}
$$

We have placed $R^{2}$ in place of $d^{2}$ in the second order terms, which is of course not strictly correct, as we should use an arithmetical mean square distance instead of a geometrical mean square distance. (See p. I7I.) Nevertheless, (86) is a much closer approximation than $(85)$.

\section{PERRY'S APPROXIMATE FORMULA}

Professor Perry has given ${ }^{92}$ the following empirical expression for the self-inductance of a short circular coil of rectangular section:

$$
L=\frac{4 \pi n^{2} a^{2}}{0.2317 a+0.44 b+0.39 c}
$$

in which $n$ is the whole number of turns of wire, $a$ the mean radius, $b$ the axial breadth, $c$ the radial depth. As in all the formulas of this paper, the dimensions are in centimeters and the value of $L$ is in centimeters. This formula gives a good approximation to $L$ as long as $b$ and $c$ are small compared with $a$. 


\section{WEINSTEIN'S FORMULA}

Maxwell's more accurate expression for the self-inductance of a circular coil of rectangular section ${ }^{93}$ was not quite correct. The investigation was repeated by Weinstein,${ }^{94}$ who gave the following formula:

where

$$
L_{u}=4 \pi a n^{2}(\lambda+\mu)
$$

$$
\begin{aligned}
& \lambda=\log \frac{8 a}{c}+\frac{\mathrm{I}}{\mathrm{I} 2}-\frac{\pi x}{3}-\frac{\mathrm{I}}{2} \log \left(\mathrm{I}+x^{2}\right)+\frac{\mathrm{I}}{\mathrm{I} 2 x^{2}} \log \left(\mathrm{I}+x^{2}\right) \\
& +\frac{\mathrm{I}}{\mathrm{I} 2} x^{2} \log \left(\mathrm{I}+\frac{\mathrm{I}}{x^{2}}\right)+\frac{2}{3}\left(x-\frac{\mathrm{I}}{x}\right) \tan ^{-1} x \\
& \mu=\frac{c^{2}}{96 a^{2}}\left[\left(\log \frac{8 a}{c}-\frac{\mathrm{I}}{2} \log \left(\mathrm{I}+x^{2}\right)\right)\left(\mathrm{I}+3 x^{2}\right)+3 \cdot 45 x^{2}+\frac{22 \mathrm{I}}{6 \mathrm{o}}\right. \\
& \left.-\mathrm{I} .6 \pi x^{3}+3.2 x^{3} \tan ^{-1} x-\frac{\mathrm{I}}{\mathrm{IO}} \frac{\mathrm{I}}{x^{2}} \log \left(\mathrm{I}+x^{2}\right)+\frac{\mathrm{I}}{2} x^{4} \log \left(\mathrm{I}+\frac{\mathrm{I}}{x^{2}}\right)\right]
\end{aligned}
$$

$b$ and $c$ are the breadth and depth of the coil and $x=\frac{b}{c}$.

Weinstein's formula for the case of a square section, where $b=c$ reduces to the following simpler expression:

$$
L_{u}=4 \pi a n^{2}\left\{\left(\mathrm{I}+\frac{c^{2}}{24 a^{2}}\right) \log \frac{8 a}{c}+.03657 \frac{c^{2}}{a^{2}}-\mathrm{I} .194914\right\}
$$

This is a very accurate formula as long as $c / a$ is a small quantity. The current is supposed distributed uniformly over the section of the coil, and hence for a winding of round insulated wire, correction must be made by formula (93).

\section{STEFAN'S FORMULA}

Stefan $^{95}$ simplified Weinstein's expression (88) by collecting together terms depending on the ratio of $b$ to $c$ and computing two short tables of constants $y_{1}$ and $y_{2}$. His formula is as follows:

$$
L=4 \pi a n^{2}\left\{\left(\mathrm{I}+\frac{3 b^{2}+c^{2}}{96 a^{2}}\right) \log \frac{8 a}{\sqrt{b^{2}+c^{2}}}-y_{1}+\frac{b^{2}}{\mathrm{I} 6 a^{2}} y_{2}\right\}
$$

${ }^{93}$ Phil. Trans., I865, and Collected Works.

94 Wied. Annal., 21, p. 329; I884.

${ }^{95}$ Wied. Annal., 22, p. II3; I884. 
The values of $y_{1}$ and $y_{2}$ are given in Table VI, page 196 , as functions of $x=b / c$ or $c / b$; that is, $x$ is the ratio of the breadth to the depth of the section, or vice versa, being always less than unity. This formula must be corrected by the quantity $\Delta_{2} L$ as shown below.

For the method of taking the dimensions $b$ and $c$ of the cross section, see page II6, section 6; also example 47, page 97.

\section{LONG COIL OF RECTANGULAR SECTION; I. E., SOLENOID OF MORE THAN ONE LAYER}

ROSA'S METHOD

When the coil is so long that the formula of Stefan 1s no longer accurate, the self-inductance may be accurately calculated by a method given by Rosa. ${ }^{96}$

In Figs. 39, 40, and 4I are shown three coils, having the same

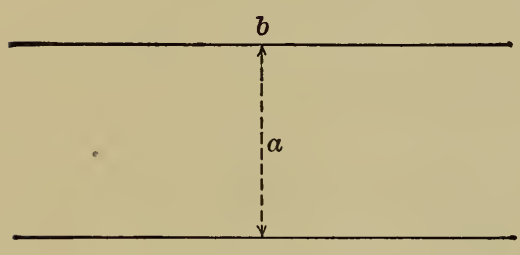
length and mean radius. The first is a single winding of thin tape and the self-inductance, calculated by a current sheet formula, is $L_{s}$. The second is a single layer of wire of square section (length $b$, depth $c$, and $b / c$ turns) and its selfinductance is $L_{u}$, the current being supposed uniformly distributed over the area of the square conFig. 39 ductors. The third is a winding of round insulated wire of length $b$, depth $c$, and any number of layers, and its self-inductance is $L$. These different self-inductances are related as follows:

$$
\begin{gathered}
L_{s}-\Delta_{1} L=L_{u} \\
L_{u}+\Delta_{2} L=L \\
\therefore L=L_{s}-\Delta_{1} L+\Delta_{2} L
\end{gathered}
$$

$L_{s}$ is calculated by any current sheet formula as (69), (7I), (72), or (73). The correction $\Delta_{1} L$ for the depth of the coil is given by the following formula:

$$
\Delta_{1} L=4 \pi a n^{\prime}\left[A_{s}+B_{s}\right]
$$


This formula has the same form as (80), but some of the quantities have a different meaning; $a$ is the mean radius as before, $n^{\prime}$ is $b / c$, the number of square conductors in the length $b$, Fig. 40, and $A_{s}$ and $B_{s}$ are given in Tables IX and X.
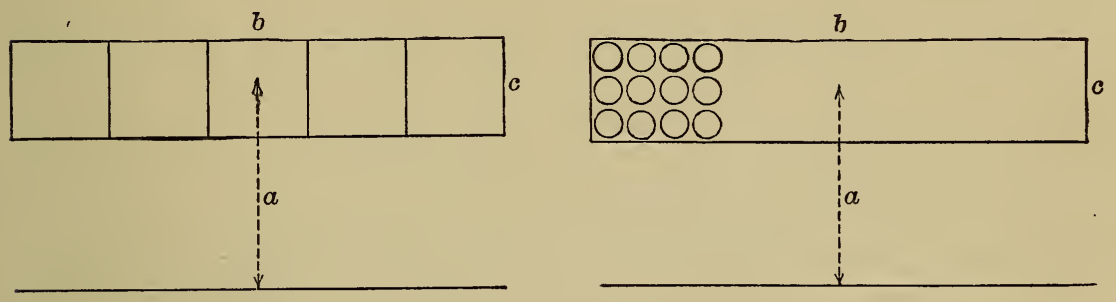

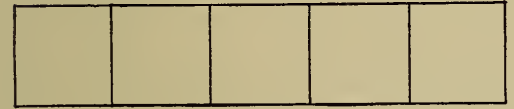

Fig. 40

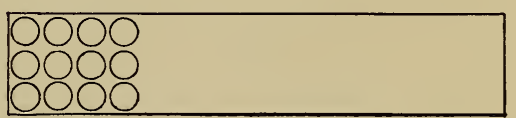

Fig. 41

The correction $\Delta_{2} L$ is calculated in precisely the same way as for a short coil, as described below, formula (93). The above formula for $\Delta_{1} L$ gives a very accurate value of the correction to be applied to $L_{s}$ to obtain $L_{u}$, and permits a test to be made for the error of Stefan's formula when applied to longer coils than the latter is intended for. Such a calculation shows that for a coil as long as its diameter Stefan's formula (and Weinstein's also, of course) is I per cent in error, giving too large a value.

\section{COHEN'S APPROXIMATE FORMULA}

Cohen has given the following approximate formula ${ }^{97}$ for the selfinductance of a long coil or solenoid of several layers: 


$$
\begin{aligned}
L=\Delta \pi^{2} n^{2} m\left\{\frac{2 a_{0}^{4}+a_{0}^{2} l^{2}}{\sqrt{4 a_{0}^{2}+l^{2}}}-\frac{8 a_{0}^{3}}{3 \pi}\right\} \\
+8 \pi^{2} n^{2}\left[\left\{(m-\mathrm{I}) a_{1}^{2}+(m-2) a_{2}^{2}+\cdots\right\}\left(\sqrt{a_{1}^{2}+l^{2}}-\frac{7}{8} a_{1}\right)\right. \\
+\frac{\mathrm{I}}{2}\left\{m(m-\mathrm{I}) a_{1}^{2}+(m-\mathrm{I})(m-2) a_{2}^{2}+\cdots\right\}\left(\frac{a_{1} \delta a}{\sqrt{a_{1}^{2}+l^{2}}}-\delta a\right) \\
\left.\quad-\frac{\mathrm{I}}{2}\left\{m(m-\mathrm{I}) a_{1}{ }^{2}+(m-2)(m-3) a_{2}{ }^{2} \cdots\right\} \frac{\delta a}{8}\right] \quad\left[9^{2}\right]
\end{aligned}
$$

where $a_{0}$ is the mean radius of the solenoid, $a_{1}, a_{2}, \cdots a_{m}$ are the mean radii of the various layers in the order of their magnitudes, $m$ is the number of layers and $\delta a$ is the distance between centers for any two consecutive layers, and $n$ is the number of turns per unit length.

For long solenoids, where the length is, say, four times the diameter, we can neglect the last term in equation (92).

This formula is sufficiently accurate for most purposes; it will give results accurate to within one-half of I per cent even for short solenoids, where the length is only twice the diameter.

\section{MAXWELL'S CORRECTIOIN FORMULA ${ }^{98}$}

GIVING THE VALUE OF $\Delta_{2} L$

Maxwell has shown that when a coil of rectangular section (Fig. $4 \mathrm{I}$ ) is wound with round insulated wire and the self-inductance is calculated by a formula in which the current is assumed to be distrib-

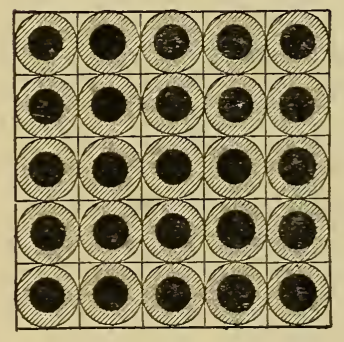

Fig. 42 uted uniformly over the section, as in Weinstein's and Stefan's, the calculated value $L_{u}$ is subject to three corrections, each of which tends to increase the calculated value of the self-inductance. Thus:

$$
L=L_{u}+\Delta_{2} L
$$

and $\Delta_{2} L=4 \pi a n\left\{\log _{e} \frac{D}{d}+\right.$ o. I $\left.3806+E\right\}$

Maxwell showed that the first term takes account of the effect of the insulation, $d$ and $D$ being the diameters of the bare and covered wire, respectively, Fig. 42. The second correction term (0.13806) 
reduces from a square section to a circular section for the conductor. The third correction term $E$ takes account of the differences in the mutual inductances of the separate turns of wire on one another when the wire has a round section from what the mutual inductances would be if the wire were of square section and no space was occupied by insulation. This term was stated by Maxwell to be equal to - o.oI97 I it was subsequently stated by Stefan to be equal to + o.or688. Rosa has shown ${ }^{99}$ that its value is variable, depending on the number of turns of wire in the coil and the shape of the cross section of the latter, and has given the values of $E$ for a number of particular cases.

From the following table one can interpolate for $E$ for any particular case not included in the table.

Summary of the values of $E$ found for the various cases considered:

\begin{tabular}{|c|c|c|c|c|}
\hline \multicolumn{3}{|c|}{2 turns } & $E=$ & 0.006528 \\
\hline 3 & “ & (one layer) & $E=$ & .009045 \\
\hline 4 & " & (two layers) & $E=$ & .01691 \\
\hline 4 & " & (one layer) & $E=$ & .01035 \\
\hline 8 & " & (two layers) & $E=$ & .OI 335 \\
\hline IO & " & (one layer) & $E=$ & .or 276 \\
\hline 20 & " & (one layer) & $E=$ & .or 357 \\
\hline I6 & " & (four layers) & $E=$ & .01512 \\
\hline IOO & " & (ten layers) & $E=$ & .01713 \\
\hline 400 & "6 & $(20 \times 20)$ & $E=$ & .01764 \\
\hline $\mathrm{I}, \mathrm{OOO}$ & " & $(50 \times 20)$ & $E=$ & .01778 \\
\hline Infinite & aur & aber of turn & $E=$ & .01806 \\
\hline
\end{tabular}

The correction $\Delta_{2} L$ is much smaller than $\Delta_{1} L$, and can be neglected except when the highest accuracy is sought. The value

${ }^{99}$ This Bulletin, 3, p. 37 ; 1907.

$21674^{\circ}-12-10$ 
of $L_{s}$ and $\Delta_{1} L$ can be calculated with accuracy if the dimensions are accurately known, and this is possible if one uses enameled wire of uniform section and takes proper care in winding and measuring the coil. However, such a coil can not be recommended for a standard of the highest precision, and the full theory is given for the sake of completeness and to show the magnitude of the smaller corrections, rather than because all the corrections are likely to be generally needed in practice.

\section{CHOICE OF FORMULAS}

If the dimensions of the cross section be very small relatively to the mean radius, formula (86) may be used. Formula (85) is a still rougher approximation, as is also (87).

For somewhat larger cross section Weinstein's formula (88) will give good results. Stefan's form (90) of Weinstein's expression is more convenient to use. Formula (89) is convenient and accurate for coils of square cross section. All these formulas assume that the current is uniformly distributed over the cross section of the coil, and must consequently be corrected by formula (93) to reduce to a winding of round wires.

The formulas (88) and (90) begin to be in error for long coils. Cohen's formula (92), however, is most accurate for long solenoids, whose length is more than about four times the diameter.

The most accurate formulas are those of Rosa's method (9I) and (93). Since the current sheet value may be very accurately obtained by any of the suitable formulas in section 6 , this method may be applied to any solenoidal coil whatever.

\section{EXAMPLES ILLUSTRATING THE FORMULAS FOR THE SELF-INDUCTANCE OF CIRCULAR COILS OF RECTANGULAR SECTION}

\section{EXAMPLE 64. MAXWELL'S APPROXIMATE FORMULAS (85), (86) AND PERRY'S APPROXIMATE FORMULA (87) COMPARED WITH WEIN- STEIN'S FORMULA (89)}

Suppose a coil of mean radius $4 \mathrm{~cm}$, with roo turns of insulated wire, wound in a square channel $I \times I \mathrm{~cm}$. (Fig. 43.)

Substituting in (85) $a=4, n=\mathrm{IO}, R=0.44705$ (the g. m. d. of a square $\mathrm{I} \mathrm{cm}$ on a side) we have 


$$
\begin{aligned}
L & =4 \pi \cdot 4 \cdot \overline{\mathrm{IOO}}^{2}\left[\log _{e} \frac{32}{\cdot 44705}-2\right] \\
& =\text { I.I } 4 \text { I millihenrys. }
\end{aligned}
$$

This is a first approximation to the self-inductance of the coil. Formula (86) gives a second approximation as follows:

$$
\begin{aligned}
L & =4 \pi .4 \cdot \overline{\mathrm{IOO}}^{2}\left[\log _{e} \frac{32}{0.44705}\left(\mathrm{I}+\frac{3 \times \overline{\mathrm{0.447}}^{2}}{25^{6}}\right)-\left(2+\frac{\overline{0.447}^{2}}{25^{6}}\right)\right] \\
& =\mathrm{I} .146 \text { millihenrys. }
\end{aligned}
$$

Perry's approximate formula, which applies only to relatively short coils, happens to give a very close approximation for this case. Substituting in (87), the above values, and also $b=c=\mathrm{I}$,

$$
\begin{aligned}
L & =\frac{4 \pi \overline{\mathrm{IOO}}^{2} \times \mathrm{I} 6}{0.9268+0.44+0.39} \\
& =\mathrm{I} . \mathrm{I} 44 \text { millihenrys. }
\end{aligned}
$$

Substituting in the more accurate formula (89) of Weinstein we shall obtain a value with which to compare the above approximations.

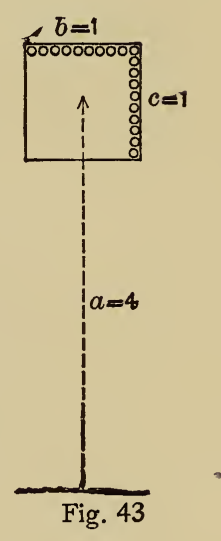

$$
\begin{aligned}
L & =\mathrm{I} 60000 \pi\left[\left(\mathrm{I}+\frac{\mathrm{I}}{384}\right) \log _{e} \frac{32}{\mathrm{I}}+0.03657 \times \frac{\mathrm{I}}{\mathrm{I} 6}-\mathrm{I} . \mathrm{I} 949 \mathrm{I} 4\right] \\
& =\mathrm{I} . \mathrm{I} 47 \text { millihenrys. }
\end{aligned}
$$

For $a=4, b=2, c=\mathbf{I} \quad n=200$

Formula (85) gives 3.750 millihenrys

$\begin{array}{lllll}\text { " } & (86) & \text { " } & 3.787 & \text { " } \\ \text { " } & (87) & \text { " } & 3.66 \mathrm{I} & \text { " } \\ & (89) & \text { “ } & 3.805 & \end{array}$

For $a=\mathrm{IO}, \quad b=\mathrm{I}, \quad c=\mathrm{I}, \quad n=\mathrm{I00}$

Formula (85) gives 4.005 millihenrys

$\begin{array}{lllll}\text { " } & (86) & \text { " } & 4.007 & \text { " } \\ \text { " } & (87) & \text { " } & 3.994 & \text { " } \\ & (89) & \text { " } & 4.008 & \end{array}$

It will be seen that formula (87) does not give as close approximations as the others, except in the case of the first example, where it 
happens to give a value very close to that given by (89). All the values, those of (89) included, are subject to correction by (93) when the coil is wound with round insulated wire.

\section{EXAMPLE 65. FORMULAS (89) AND (90) COMPARED WITH CURRENT- SHEET FORMULAS}

As a test of these formulas we may calculate the self-inductance of a single turn of wire, using the case already calculated in example b 52 ; that is, a circle of radius $a=25 \mathrm{~cm}$, and the diameter of $0.1 \mathrm{~cm}$ the bare wire is I $\mathrm{mm}$. Substituting these values in (89) we have

$$
\begin{aligned}
L & =100 \pi\left[\left(\mathrm{I}+\frac{.0 \mathrm{I}}{\mathrm{I} 5000}\right) \log _{e} 2000+\frac{.03657}{(250)^{2}}-\mathrm{I} . \mathrm{I} 949 \mathrm{I} 4\right] \\
& =640.5995 \pi \mathrm{cm} .
\end{aligned}
$$

Substituting in (90),

$$
\begin{aligned}
L=\mathrm{IOO} \pi & {\left[\left(\mathrm{I}+\frac{. \mathrm{OI}}{\mathrm{I} 5000}\right) \log _{e} \frac{2 \mathrm{OO}}{\sqrt{.02}}-0.848340+\frac{.0 \mathrm{I} \times .8 \mathrm{I} 62}{\mathrm{IO000}}\right] } \\
& =640.5995 \pi \mathrm{cm},
\end{aligned}
$$

- agreeing with the value by (89).

Fig. 44 These values are for a conductor of square cross section (Fig. 44). To reduce to a circular section of same diameter (0.1 cm) we must apply the second correction term of (93); that is, add to the above value

$$
\begin{aligned}
& \Delta L=4 \pi a \times 0.138060 \\
& \text { Thus, } L=(640.5995+\mathrm{I} 3.8060) \pi \\
& =654.4055 \pi \mathrm{cm} \text {, }
\end{aligned}
$$

which agrees with the value found for the self-inductance of a round wire $0.1 \mathrm{~cm}$ diameter, bent into a circle of $25 \mathrm{~cm}$ radius, by formula (63) example $5^{2}$ and formulas (69) and (80), example $5^{8 .}$

EXAMPLE 66. STEFAN'S FORMULA (90) COMPARED WITH (69) BY MEANS OF ROSA'S CORRECTION FORMULA (91)

Suppose a coil of mean radius $10 \mathrm{~cm}$, wound with roo turns in a square channel $\mathrm{I} \times \mathrm{I} \mathrm{cm}$. Assuming the current uniformly distributed we obtain from (90), in which $y_{1}=0.848340, y_{2}=0.8162$, 


$$
\begin{aligned}
& \log _{e} \frac{8 a}{\sqrt{b^{2}+c^{2}}}=\log _{e} \frac{80}{\sqrt{2}}=4.03545 \\
& L_{u}=4 \pi \times 100,000\left[\left(\mathrm{I}+\frac{4}{9600}\right) 4.03545-0.84834+0.0005 \mathrm{I}\right] \\
& =4 \pi \times 318,930 \mathrm{~cm} \\
& =4.00779 \text { millihenrys. }
\end{aligned}
$$

By formula (69) we have for the self-inductance of a current sheet for which $a=\mathrm{IO}, b=\mathrm{I}, n=\mathrm{I}$,

$$
L_{s}=4 \pi \times 38.83475 \mathrm{~cm} \text {. }
$$

This is larger than the value for the coil of section $\mathrm{I} \times \mathrm{I}$ by $\Delta_{1} L$, the value of the latter being given by formula (9I).

By Table IX, $A_{s}=0.6942$. More closely, it is $0.694 \mathrm{I} 5 \cdot{ }^{100}$

By Table $\mathrm{X}, B_{s}=0$. In this case $n^{\prime}=\mathrm{I}$. Hence,

$$
\begin{aligned}
& \Delta_{1} L=4 \pi \times 10 \times 0.694 \mathrm{I} 5=4 \pi \times 6.94 \mathrm{I} 5 \mathrm{~cm} \\
& \therefore L_{1}=4 \pi(38.83475-6.94 \mathrm{I} 5)=400.782 \mathrm{~cm} .
\end{aligned}
$$

This is the value of the self-inductance for one turn only, the current being uniformly distributed. For roo turns $L$ is Io $^{4}$ times as great.

$$
\therefore L_{u}=4.00782 \text { millihenrys. }
$$

This value agrees with the above value by Stefan's formula within less than one part in one hundred thousand.

For a coil of insulated round wires, this result must be corrected by formula (93).

For a coil of the same radius, but of length $b=10 \mathrm{~cm}, c=\mathrm{I} \mathrm{cm}$, wound with ro layers of Ioo turns each, we have the following values:

By Stefan's formula, $y_{1}=0.59243, y_{2}=0.1325$

$$
\begin{aligned}
L_{u} & =4 \pi \times \mathrm{IO} \times \overline{\mathrm{IOOO}}^{2} \times \mathrm{I} .55536 \\
& =\mathrm{I} 95.452 \text { millihenrys. }
\end{aligned}
$$


By (69) the current sheet value of $L$ for Io turns is

$$
\begin{aligned}
L_{10} & =4 \pi \times 10 \times 100 \times 1.65095 \\
& =4 \pi \times 1650.95 .
\end{aligned}
$$

The correction for depth of section by (9I) is, since by Tables IX and X, $A_{s}=0.6942, B_{s}=0.2792$, and therefore $A_{s}+B_{s}=0.9734$

$$
\begin{aligned}
& \Delta_{1} L=4 \pi \mathrm{IO} \times \text { I0 } \times 0.9734 \\
& =4 \pi \times 97.34 \\
& \therefore L_{u}=L_{10}-\Delta_{1} L=4 \pi(1650.95-97.34) \\
& =4 \pi \times 1553.6 \mathrm{r} \mathrm{cm} \text { for } 10 \text { turns. }
\end{aligned}
$$

For $n=1000$ turns the self-inductance will be $\overline{\mathrm{IOO}}^{2}$ times as great.

$$
\begin{aligned}
L_{u} & =4 \pi \times \mathrm{I} 5.536 \mathrm{I} \times 10^{6} \mathrm{~cm} \\
& =195.232 \text { millihenrys. }
\end{aligned}
$$

This value is about I part in 900 smaller than the above value, showing that Stefan's formula gives too large results by that amount for a coil of this length. If the coil were twice as long, the error would be about ten times as great.

It is interesting to obtain by this method an estimate of the error by Stefan's formula for coils longer than those for which it is intended. For short coils it is seen to be very accurate, subject always to the corrections of formula (93), and for longer coils it gives a good approximation. The method of (9I), however, applies to coils of any length.

EXAMPLE 67. STEFAN'S FORMULA (90) COMPARED WITH (81) AND WITH STRASSER'S (82) FOR COILS OF FEW TURNS, USING THE CORRECTION FORMULA (93)

Coil of 2 turns of wire, $0.4 \mathrm{~mm}$ diameter, wound in a circle of I. $46 \mathrm{~cm}$ radius with a pitch of $2 \mathrm{~mm}$. Stefan's formula assumes a uniform distribution over a rectangular section. Suppose a section as shown in Fig. $45,4 \times 2 \mathrm{~mm}$, with one turn of wire in the center of each square. For the rectangular section, with the current uniformly distributed, the self-inductance by Stefan's formula is with $a=\mathrm{I} .46, c / b=0.5, y_{1}=0.7960, y_{2}=0.3066, L_{u}=4 \pi a n^{2} \times$ $2.4763=4 \pi a n \times 4.9526, n$ being 2 . To reduce this to the case of a 
winding of 2 turns of wire as shown we must apply the corrections given by (93) thus:

$$
\begin{aligned}
& \log D / d=\log _{e} 5=\mathrm{I} .60944 \\
& \text { second term }=0.3_{3806} \\
& \text { third term } E=\frac{0.00653}{\mathrm{I} .7540} \\
& \therefore \Delta_{2} L=4 \pi a n \times \mathrm{I} .7540 \\
& L=L_{u}+\Delta_{2} L=4 \pi a n \times 6.7066 \\
&= 246 . \mathrm{I} \mathrm{cm.}
\end{aligned}
$$

By the summation formula (8I) we have in this case

$$
\begin{aligned}
L & =2 L_{1}+2 M_{12} \\
& =4 \pi a[9.2400+4.1606] \\
& =245.86 \mathrm{~cm} .
\end{aligned}
$$

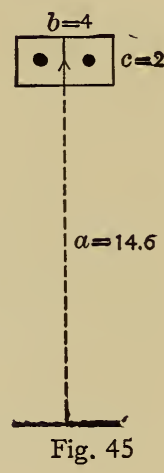

The value by Strasser's formula is the same as by the summation formula to which it is equivalent. We have also used formulas (69) and (80) for this case and have obtained 246.o.

This is one of several problems calculated by Drude ${ }^{101}$ by Stefan's formula. Drude concluded that Stefan's formula was inapplicable to such coils, as it gave results from Io to 25 per cent too large. His trouble was, however, due to taking the length of the coil as the distance between the center of the first wire and the center of the last (instead of $n$ times the pitch) and neglecting the correction terms of formula (93). As we have seen above, Stefan's formula when properly used can be depended upon to give accurate results for short coils, and results within less than I per cent for coils of length equal to the radius of the coil.

We have calculated several other cases given by Drude and give below the results, together with his experimental values. The radius is the same in each case, and the numbers in the first column are the number of turns in the several coils. 


\begin{tabular}{c|c|c|c|c}
\hline $\mathbf{n}$ & $\begin{array}{c}\text { By Stefan's Formula } \\
(90) \text { and (93) }\end{array}$ & $\begin{array}{c}\text { By Rayleigh's } \\
\text { Formula (69) and (80) }\end{array}$ & $\begin{array}{c}\text { By Strasser's } \\
\text { Formula (82) or (81) }\end{array}$ & $\begin{array}{c}\text { Drude's Observed } \\
\text { Values } \\
\text { (Values of } L \text { in } \\
\text { Centimeters) }\end{array}$ \\
\hline 2 & 246.1 & 246.0 & 245.9 & 238.5 \\
4 & 711.9 & 711.1 & 710.8 & 697.9 \\
6 & 1298.7 & 1297.7 & 1297.8 & 1271.4 \\
9 & 2318.0 & 2313.0 & 2315.7 & 2300.1 \\
\hline
\end{tabular}

It will be seen that the values by the different formulas agree very closely, and that the experimental values agree as closely as could be expected for such small inductances.

EXAMPLE 68. FORMULAS (69) AND (80) COMPARED WITH (90) AND (93) FOR COIL OF 20 TURNS WOUND WITH A SINGLE LAYER

$$
a=25 \quad b=2 \mathrm{~cm} \quad c=0.1 \mathrm{~cm} \quad n=20 .
$$

Diameter of bare wire $0.6 \mathrm{~mm}$, of covered wire $1.0 \mathrm{~mm}$.

In the last case we obtained the self-inductance of the coil by two distinct methods, the first being the method of summation, the second by assuming the current uniformly distributed over the section, and then applying the three corrections $C, F, E$. In this problem we may first calculate $L$ by use of the current sheet formula (69), and then apply the corrections for section, $A$ and $B$ formula (8o); and, second, by Stefan's formula for uniform distribution, and apply the three corrections $C, F, E$, which give the value for a winding of round insulated wires.

Rayleigh's formula for this example gives:

$$
\begin{gathered}
L=4 \operatorname{man}^{2}\left\{\begin{aligned}
\left.\log _{e} \mathrm{IOO}-0.5+\frac{4}{20,000}\left(\log _{e} \mathrm{IOO}+\frac{\mathrm{I}}{4}\right)\right\} \\
\log _{e} \mathrm{IOO}=4.605 \mathrm{I} 7 \mathrm{O}
\end{aligned}\right. \\
\begin{aligned}
\frac{4}{20,000}\left(\log _{e} \mathrm{IOO}+\frac{\mathrm{I}}{4}\right) & =\frac{0.00097 \mathrm{I}}{4.606 \mathrm{I} 4 \mathrm{I}} \\
& -\frac{0.500000}{4.106 \mathrm{I} 4 \mathrm{I}}
\end{aligned} \\
4 \pi a n^{2}=40,000 \pi \quad \therefore L_{s}=\mathrm{I} 64245.64 \pi \mathrm{cm} .
\end{gathered}
$$

This is the self-inductance of a winding of 20 turns of infinitely thin tape, each turn being I $\mathrm{mm}$ wide, with edges touching without 
making electrical contact, which arrangement fulfills the conditions of a current sheet. To reduce this to the case of round wires we must apply the corrections $A$ and $B$ for self and mutual induction. ${ }^{102}$

$$
\begin{gathered}
\text { By Table VII, for } d / D=0.6, A=0.0460 \\
\text { By Table VIII, for } n=20, \quad B=0.2974 \\
A+B=0.3434 \\
4 \pi a n=\quad 2,000 \pi \\
\Delta L=4 \pi a n(A+B)=\quad 686.8 \pi \mathrm{cm} \\
L=L_{s}-\Delta L=163558.84 \pi \mathrm{cm} .
\end{gathered}
$$

By Stefan's formula we find, sustituting the above values of $a$, $n, b, c$, and taking $y_{1}=0.548990$ and $y_{2}=0.1269$

$$
L_{u}=\mathrm{I} 62234.60 \pi \mathrm{cm} \text {. }
$$

The correction $E$ for a single layer coil of 20 turns is given on page I4I. The three corrections are then as follows:

$$
\begin{gathered}
C=0.13806 \\
F=0.51082=\log _{e} \frac{\text { Io }}{6} \\
E=0.01357 \\
\text { Sum }=\frac{0.66245}{\therefore \Delta L=4 \pi a n(C+F+E)=\mathrm{I} 324.90 \pi \mathrm{cm} .} \\
\therefore L=L_{u}+\Delta L=\mathrm{I} 63559.50 \pi \mathrm{cm} .
\end{gathered}
$$

This value of $L$ is greater than the value found by the other method by only four parts in a million. Thus we see that the method of calculating $L_{u}$ by Stefan's or Weinstein's formula and applying the corrections $C, F, E$ gives practically identical results with the method of summation and also with the current sheet method for short coils. When, however, the coils are longer, the agreement is not so good, for the reason that the formula of Weinstein (and Stefan's, derived from it) is not as accurate when the section of the coil is greater. Thus if the coil in the above problem had been $5 \mathrm{~cm}$ long and $2.5 \mathrm{~mm}$ deep and wound with 20 turns of heavier wire, the difference would have been one part in twenty-five thousand (still very good agreement), and if it were $10 \mathrm{~cm}$ long and 
$0.5 \mathrm{~cm}$ deep (the radius being $25 \mathrm{~cm}$ ) it would have been one part in two thousand two hundred. For most experimental work, therefore, Stefan's formula is amply accurate.

EXAMPLE 69. COHEN'S FORMULA (92) COMPARED WITH (91)

A solenoid of length $l=50 \mathrm{~cm}$, mean radius $5 \mathrm{~cm}$, depth of winding $0.4 \mathrm{~cm}$, is wound with 4 layers of wire of 500 turns each. Substituting these values in (92) we have $(n=10)$

$$
\begin{aligned}
L_{s} & =\mathrm{r} 6 \pi^{2} n^{2}\left(\mathrm{I} 144.3+333^{6.0}-\mathrm{ro.} 84-\mathrm{r} .04\right) \\
& =70.562 \text { millihenrys. }
\end{aligned}
$$

By the second method we first find $L_{s}$ by (69), then $\Delta_{1} L$ by (9I), and $\Delta_{2} L$ by $(93)$

$$
\begin{aligned}
L_{s} & =72.648 \text { millihenrys } \\
-\Delta_{1} L & =-2.167 \quad \text { " } \\
\Delta_{2} L & =\frac{0.048}{70.529} \quad \text { " }
\end{aligned}
$$

This shows a very close agreement between (92) and (9I).

In calculating $L_{s}$ we may use Table IV. Since $d / l=0.2$

$$
\begin{aligned}
Q & =3.6324, \quad a n^{2}=5 \times \overline{2000}^{2}=20,000,000 \\
L_{s} & =3.6324 \times 20,000,000 \mathrm{~cm}
\end{aligned}
$$

or,

$$
L_{8}=72.648 \text { millihenrys. }
$$

\section{SELF AND MUTUAL INDUCTANCE OF LINEAR CONDUCTORS ${ }^{103}$}

\section{SELF-INDUCTANCE OF A STRAIGHT CYLINDRICAL WIRE}

The self-inductance of a length $l$ of straight cylindrical wire of radius $\rho$ is

$$
\begin{aligned}
L & =2\left[l \log \frac{l+\sqrt{l^{2}+\rho^{2}}}{\rho}-\sqrt{l^{2}+\rho^{2}}+\frac{l}{4}+\rho\right] \\
& =2 l\left[\log \frac{2 l}{\rho}-\frac{3}{4}\right] \text { approximately. }
\end{aligned}
$$

Where the permeability of the wire is $\mu$, and that of the medium outside is unity, (95) appears in the form

$$
L=2 l\left[\log \frac{2 l}{\rho}-\mathrm{I}+\frac{\mu}{4}\right]
$$


This formula was originally given by Neumann.

For a straight cylindrical tube of infinitesimal thickness, or for alternating currents of great frequency, when there is no magnetic field within the wire, the self-inductance is

$$
L=2 l\left[\log \frac{2 l}{\rho}-\mathrm{I}\right]
$$

This is obtained by subtracting from (95) $l / 2$ or from (96) $\mu l / 2$, the magnetic flux within the conductor due to unit current.

\section{THE MUTUAL INDUCTANCE OF TWO PARALLEL WIRES}

The mutual inductance of two parallel wires of length $l$, radius $\rho$, and distance apart $d$ is the number of lines of force, due to unit current in one, which cut the other when the current disappears.

This is

$$
\begin{array}{r}
M=2\left[l \log \frac{l+\sqrt{l^{2}+d^{2}}}{d}-\sqrt{l^{2}+d^{2}}+d\right] \\
\therefore M=2 l\left[\log \frac{2 l}{d}-\mathrm{x}+\frac{d}{l}\right] \text { approximately }
\end{array}
$$

when the length $l$ is great in comparison with $d$.

Equation (98), which is an exact expression when the wires have no appreciable cross section, is not an exact expression for the mutual inductance of two parallel cylindrical wires, but is not appreciably in error even when the section is large and $d$ is small if $l$ is great compared with $d$.

\section{THE SELF-INDUCTANCE OF A RETURN CIRCUIT}

If we have a return circuit of two parallel wires each of length $l$ (the current then flowing in opposite direction in the two wires) the self-inductance of the circuit, neglecting the effect of the end connections shown by dotted lines, Fig. 46, will be very approximately

$$
L=4\left[\left[\log \frac{d}{\rho}+\frac{\mu}{4}-\frac{d}{l}\right]\right.
$$

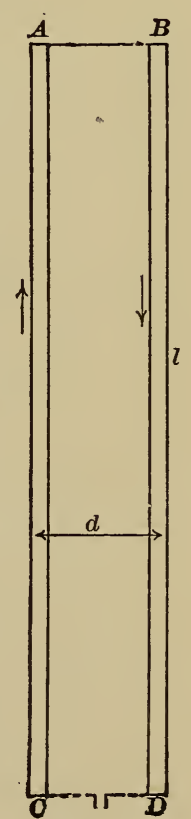

Fig. 46 
In the usual case of $\mu=\mathrm{I}$ this will be, when $d / l$ is small

$$
L=4 l\left[\log \frac{d}{\rho}+\frac{\mathrm{I}}{4}\right]
$$

If the end effect is large, as when the wires are relatively far apart, use the expression for the self-inductance of a rectangle below (107); or, better, add to the value of (IOO) the self-inductance of $\mathrm{AB}+\mathrm{CD}$, using equation (94) in which $l=2 \mathrm{AB}$.

Experimental work at the Bureau of Standards, not yet published, has shown that formula (100), and therefore (94) and (98) are consistent with the formula $\left(6_{3}\right)$ for the inductance of a circular ring.

[This is equivalent to the following formula in which the logarithms are common:

$L=0.74 \mathrm{I}$ I $\log _{10} \frac{d}{\rho}+.0805$ in millihenrys per mile of conảuctor,

$=0.4605 \log _{10} \frac{d}{\rho}+.050$ in millihenrys per kilometer of conductor. $d$ and $\rho$ being expressed in centimeters, inches, or any other unit.] MUTUAL INDUCTANCE OF TWO LINEAR CONDUCTORS IN THE SAME STRAIGHT LINE

The mutual inductance of two adjacent linear conductors of lengths $l$ and $m$ in the same straight line is

$$
M_{l m}=l \log \frac{l+m}{l}+m \log \frac{l+m}{m}, \text { approximately. }
$$

This approximation is very close indeed if the radius of the conductor (which has been assumed zero) is very small.

\section{THE SELF-INDUCTANCE OF A STRAIGHT RECTANGULAR BAR}

The self-inductance of a straight bar of rectangular section is, to within the accuracy of the approximate formula (99), the same as the mutual inductance of two parallel straight filaments of the same length separated by a distance equal to the geometrical mean distance of the cross section of the bar. Thus,

$$
L=2 i\left[\log \frac{2 l}{R}-\mathrm{I}+\frac{R}{l}\right]
$$


where $R$ is the geometrical mean distance of the cross section of the rod or bar. If the section is a square, $R=0.447 \alpha, \alpha$ being the side of the square. If the section is a rectangle, the value of $R$ is given by Maxwell's formula (124).

This is equivalent to the following:

$$
L=2 l\left[\log \frac{2 l}{\alpha+\beta}+\frac{1}{2}+\frac{0.2235(\alpha+\beta)}{l}\right]
$$

In the above formula $L$ is the self-inductance of a straight bar or wire of length $l$ and having a rectangular section of length $\alpha$ and breadth $\beta$.

\section{TWO PARALLEL BARS. SELF AND MUTUAL INDUCTANCE}

The mutual inductance of two parallel straight, square, or rectangular bars is equal to the mutual inductance of two parallel wires or filaments of the same length and at a distance apart equal to the geometrical mean distance of the two areas from one another. This is very nearly equal in the case of square sections to the distance between their centers for all distances, the $\mathrm{g}$. m. $\mathrm{d}$. being a very little
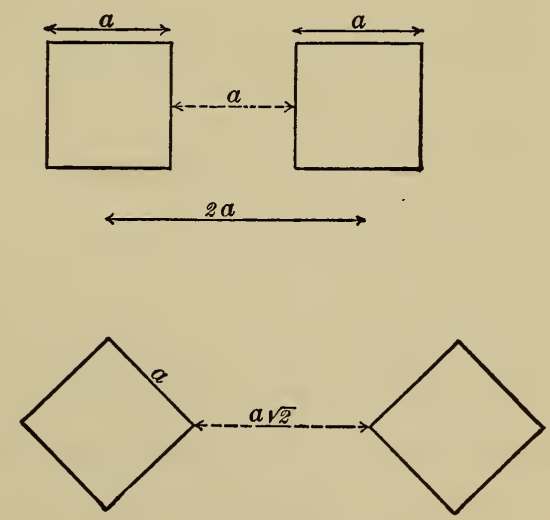

Fig. 47

greater for parallel squares, and a very little less for diagonal squares $^{104}$ (Fig. 47). We should, therefore, use equation (99) with $d$ equal to $g$. m. d. of the sections from one another; that is, substantially, to the distances between the centers. 
The self-inductance of a return circuit of two such parallel bars is equal to twice the self-inductance of one minus twice their mutual inductance. That is,

$$
L=2\left[L_{1}-M\right]
$$

in which $L_{1}$ is calculated by (104) and $M$ by (99).

\section{SELF-INDUCTANCE OF A SQUARE}

The self-inductance of a square may be derived from the expressions for the self and mutual inductance of finite straight wires from the consideration that the self-inductance of the square is the sum of the self-inductances of the four sides minus the mutual inductances. That is,

$$
L=4 L_{1}-4 M
$$

the mutual inductance of two mutually perpendicular sides being zero. Substituting $a$ for $l$ and $d$ in formulas (94) and (98) we have, neglecting $\rho^{2} / a^{2}, L=8 a\left(\log \frac{a}{\rho}+\frac{\rho}{a}-.524\right)$ where $a$ is the length of one side of the square and $\rho$ is the radius of the wire. If we put $l=4 a=$ whole length of wire in the square,

$$
\begin{gathered}
L=2 l\left(\log \frac{l}{\rho}+\frac{4 \rho}{l}-\mathrm{r} .9 \mathrm{ro}\right) \\
\text { or, } L=2 l\left(\log \frac{l}{\rho}-\mathrm{r} .9 \mathrm{ro}\right) \text { approximately. }
\end{gathered}
$$

Formulas (105) and (I06) were first given by Kirchhoff ${ }^{105}$ in I 864 .

\section{SELF-INDUCTANCE OF A RECTANGLE}

(a) The conductor having a circular section

The self-inductance of the rectangle of length $a$ and breadth $b$ is

$$
L=2\left(L_{a}+L_{b}-M_{a}-M_{b}\right)
$$

where $L_{a}$ and $L_{b}$ are the self-inductances of the two sides of length $a$ and $b$ taken alone, $M_{a}$ and $M_{b}$ are the mutual inductances of the two opposite pairs of length $a$ and $b$, respectively.

From (94) and (98) we therefore have, neglecting $\rho^{2} / a^{2}$, and putting $d$ for the diagonal of the rectangle $=\sqrt{a^{2}+b^{2}}$ 


$$
\begin{aligned}
L=4[(a+b) \log & \frac{2 a b}{\rho}-a \log (a+d)-b \log (b+d) \\
& \left.-\frac{7}{4}(a+b)+2(d+\rho)\right]
\end{aligned}
$$

(b) The conductor having a rectangular section

For a rectangle made up of a conductor of rectangular section $\alpha \times \beta$,

$$
\begin{gathered}
L=4\left[(a+b) \log \frac{2 a b}{\alpha+\beta}-a \log (a+d)-b \log (b+d)\right. \\
\left.-\frac{a+b}{2}+2 d+0.447(\alpha+\ddot{\beta})\right]
\end{gathered}
$$

where as before $d$ is the diagonal of the square. This is equivalent to Sumec's exact formula ${ }^{106}(6 a)$.

For $a=b$, a square,

$$
L=8 a\left[\log \frac{a}{\alpha+\beta}+0.2235 \frac{\alpha+\beta}{a}+0.726\right]
$$

If $\alpha=\beta$, that is, the section of the conductor is a square,

$$
L=8 a\left[\log \frac{a}{\alpha}+0.447 \frac{\alpha}{a}+0.033\right]
$$

\section{MUTUAL INDUCTANCE OF TWO EQUAL PARALLEL RECTANGLES}

For two equal parallel rectangles of sides $a$ and $b$ and distance apart $d$ the mutual inductance, which is the sum of the several mutual inductances of parallel sides, is,

$$
\begin{aligned}
M=4[a \log ( & \left.\frac{a+\sqrt{a^{2}+d^{2}}}{a+\sqrt{a^{2}+b^{2}+d^{2}}} \cdot \frac{\sqrt{b^{2}+d^{2}}}{d}\right) \\
+ & \left.b \log \left(\frac{b+\sqrt{b^{2}+d^{2}}}{b+\sqrt{a^{2}+b^{2}+d^{2}}} \cdot \frac{\sqrt{a^{2}+d^{2}}}{d}\right)\right] \\
+ & 8\left[\sqrt{a^{2}+b^{2}+d^{2}}-\sqrt{a^{2}+d^{2}}-\sqrt{b^{2}+d^{2}}+d\right][\mathrm{III}]
\end{aligned}
$$

${ }^{106}$ Elektrotech. Zs., 27, p. II75; 1906. 
For a square, where $a=b$, we have

$$
\begin{aligned}
M=8[a & \left.\log \left(\frac{a+\sqrt{a^{2}+d^{2}}}{a+\sqrt{2 a^{2}+d^{2}}} \frac{\sqrt{a^{2}+d^{2}}}{d}\right)\right] \\
+8 & {\left[\sqrt{2 a^{2}+d^{2}}-2 \sqrt{a^{2}+d^{2}}+d\right] . }
\end{aligned}
$$

Formula (III) was first given by F. E. Neumann ${ }^{107}$ in 1845 .

The case of two rectangles symmetrically placed about a common vertical axis, the horizontal sides of the smaller rectangle being equidistant from those of the larger rectangle, has been discussed by Martens ${ }^{10.8}$ and a formula derived which enables the mutual inductance to be found for any angle $\xi$ between the planes of the rectangles. This formula is, however, very elaborate and calculations therewith laborious.

\section{SELF AND MUTUAL INDUCTANCE OF THIN TAPES}

The self-inductance of a straight, thin tape of length $l$ and breacith $b$ (and of negligible thickness), Fig. 48 (I), is equal to the mutual inductance of two parallel lines of distance apart $R_{1}$ equal to the geometrical mean distance of the section, which is $0.223 \mathrm{I} 3 b$, or $\log R_{1}=\log b-\frac{3}{2}$.

Thus we have approximately

$$
\begin{aligned}
L & =2 l\left[\log \frac{2 l}{R_{1}}-\mathrm{I}\right] \\
& =2 l\left[\log \frac{2 l}{b}+\frac{\mathrm{I}}{2}\right]
\end{aligned}
$$

If the thickness of the tape is not negligible, this formula becomes, when $a$ is the thickness of the tape,

$$
L=2 l\left[\log \frac{2 l}{b}-\frac{a}{b}+\frac{I}{2}\right]
$$

A closer approximation to $L$ is given by (IO4), in which $\alpha$ is the thickness and $\beta$ is the breadth of the tape. For two such tapes in the same plane, coming together at their edges with-

${ }^{107}$ Allgemeine Gesetze der Inducirten Ströme, Abh. Berlin Akad.

${ }^{108}$ Ann. der Phys. 29, p. 963; I909. 
out making electrical contact, Fig. 48 (2), the mutual inductance is

$$
\begin{aligned}
M & =2 l\left[\log \frac{2 l}{R_{2}}-\mathrm{I}\right] \\
& =2 l\left[\log \frac{2 l}{b}-0.8863\right]
\end{aligned}
$$

where $R_{2}$ is the geometrical mean distance of one tape from the other, which in this case is $0.89252 b$. For a return circuit made up of these two tapes the self-inductance is

$$
\begin{aligned}
L & =2 L_{1}-2 M \\
& =4 l\left(\log \frac{R_{2}}{R_{1}}\right)=4 l \log _{e} 4 \quad \text { [II6] } \\
& =5.545 \times \text { length of one tape. }
\end{aligned}
$$

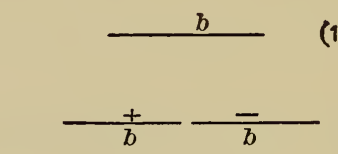

(1)

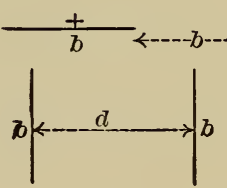

(4)

Thus the self-inductance of such a circuit is independent of the width of the tapes. If the tapes are separated by the distance $b$, Fig. 48 (3), equal to the width of the tapes, $R_{2}=1.95653 b$ and $L=8.685 l$.

If the two tapes are not in the same plane, but parallel, Fig. 48 (4),

$$
L=2 L_{1}-2 M=4 l \log \frac{R_{2}}{R_{1}}
$$

and when the distance apart is equal to the breadth of the tapes, Fig. 48 (5), we have

$$
\log \frac{R_{2}}{R_{1}}=\frac{\pi}{2}
$$

and

$$
L=4 l \frac{\pi}{2}=2 \pi l
$$

In this case, also, the self-inductance [ $2 \pi \mathrm{cm}$ per unit of length] of the pair of thin strips is independent of their width so long as the distance apart is equal to their width. Formula (II7) with (I32) $2 \mathrm{I} 674^{\circ}-\mathrm{I} 2-\mathrm{II}$ 
may be employed to calculate the self-inductance of a noninductive shunt made up of a sheet of thin metal doubled on itself.

\section{CONCENTRIC CONDUCTORS}

The self-inductance of a thin, straight tube of length $l$ and radius $a_{2}$, when $a_{2} / l$ is very small, is given by (97),

$$
L_{2}=2 l\left[\log \frac{2 l}{a_{2}}-\mathrm{I}\right]
$$

The mutual inductance of such a tube on a conductor within it is equal to its self-inductance, since all the lines of force due to the outer tube cut through the inner when they collapse on the cessation of current. The self-inductance of the inner conductor, supposed a solid cylinder, is

$$
L_{1}=2 l\left[\log \frac{2 l}{a_{1}}-\frac{3}{4}\right]
$$

If the current goes through the latter and returns through the outer tube, the self-inductance of the circuit is

since $M$ equals $L_{2}$

$$
L=L_{1}+L_{2}-2 M=L_{1}-L_{2}
$$

$$
\therefore L=2 l\left[\log \frac{a_{2}}{a_{1}}+\frac{\mathrm{I}}{4}\right]
$$

This result can also be obtained by integrating the expression for the force outside $a_{1}$ between the limits $a_{1}$ and $a_{2}$, and adding the term for the field within $a_{1}$, there being no magnetic field outside $a_{2}$.

If the outer tube has a thickness $a_{3}-a_{2}$ and the current is distributed uniformly over its cross section the self-inductance will be a little greater, the geometrical mean distance from $a_{1}$ to the tube, which is more than $a_{2}$ and less than $a_{3}$, being given by the expression

$$
\log a_{g}=\frac{a_{3}^{2} \log a_{3}-a_{2}^{2} \log a_{2}}{a_{3}^{2}-a_{2}^{2}}-\frac{\mathrm{I}}{2}
$$

Putting this value of $\log a$ in (IIg) in place of $\log a_{2}$, we should have the self-inductance of the return circuit.

If the current is alternating and of very high frequency, the current would flow on the outer surface of $a_{1}$ and on the inner surface 
of the tube, and $L$ for the circuit would be

$$
L=2 l \log \frac{a_{2}}{a_{1}}
$$

\section{MULTIPLE CONDUCTORS}

If a current be divided equally between two wires of length $l$, radius $\rho$ and distance $d$ apart, the self-inductance of the divided conductor is the sum of their separate self-inductances plus twice their mutual inductance.

Thus, when $d / l$ is small,

$$
L=2 l\left[\log \frac{2 l}{(\rho d)^{\frac{1}{2}}}-\frac{7}{8}\right]=2 l\left[\log \frac{2 l}{\left(r_{g} d\right)^{\frac{1}{2}}}-\mathrm{I}\right]
$$

where $r_{g}$, the g. m. d. of the section of the wire is $0.7788 \rho$ for a round section.

If there are three straight conductors in parallel and distance $d$ apart, the self-inductance is similarly

$$
L=2 l\left[\log \frac{2 l}{\left(r_{g} d^{2}\right)^{\frac{1}{3}}}-\mathrm{I}\right]
$$

The expression $\left(r_{g} d^{2}\right)^{\frac{1}{3}}$ is the g. m. d. of the multiple conductor.

EXAMPLES ILLUSTRATING THE FORMULAS FOR THE SELF AND MUTUAL INDUCTANCE OF LINEAR CONDUCTORS

EXAMPLE 70. FORMULAS (94), (95), (96), AND (97)

A straight copper wire $100 \mathrm{~cm}$ long and $0.2 \mathrm{~cm}$ diameter will have a self-inductance by formula (95) of

$$
L=200\left(\log _{e} \frac{200}{0.1}-\frac{3}{4}\right)=1370.18 \mathrm{~cm} .
$$

If it were twice as long

$$
L=400\left(\log _{e} \frac{400}{0.1}-\frac{3}{4}\right)=3017.62 \mathrm{~cm} .
$$

The more exact formula (94) gives practically the same result where $\rho$ is so small compared with $l$. 
If the wire were of iron with a permeability of rooo, we should have in the first case for $l=100$

$$
L=200\left(\log _{e} 2000-\mathrm{I}+250\right)=5 \mathrm{I} 320 \mathrm{~cm} .
$$

For sufficiently rapid oscillations so that the current may be considered to be confined to the surface of the wire

$$
L=200\left(\log _{e} 2000-\mathrm{I}\right)=\mathrm{I} 320.18 \mathrm{~cm} .
$$

If the length of the conductor were ro meters and the diameter $0.2 \mathrm{~cm}$ as before, the self-inductance by (95) would be

$$
\begin{aligned}
L=2000\left(\log _{e} 20000-\frac{3}{4}\right) & =18307.0 \mathrm{~cm} \\
& =\mathrm{r} 8.307 \text { microhenrys. }
\end{aligned}
$$

EXAMPLE 71. FORMULAS (98) AND (99)

Two parallel copper wires of length $100 \mathrm{~cm}$ and distance apart $200 \mathrm{~cm}$ will have a mutual inductance of

$$
\begin{aligned}
M & =2\left[\mathrm{IOO} \log _{e} \frac{\mathrm{IOO}+\mathrm{I00} \sqrt{5}}{200}-\mathrm{I00} \sqrt{5}+200\right] \\
& =200\left[\log _{e} \frac{\mathrm{I}+\sqrt{5}}{2}-\sqrt{5}+2\right] \\
& =200\left(\log _{e} \mathrm{I} .6 \mathrm{I} 803-0.236 \mathrm{I}\right) \\
& =49.02 \mathrm{~cm} .
\end{aligned}
$$

If the length of each conductor were $200 \mathrm{~cm}$ and the distance apart $100 \mathrm{~cm}$, then

$$
M=400\left[\log _{e} \frac{2+\sqrt{5}}{\mathrm{I}}-\frac{\sqrt{5}}{2}+\frac{\mathrm{I}}{2}\right]=330.24 \mathrm{~cm} .
$$

The approximate formula (99) is only applicable when the length of the conductors is great compared with their distance apart. Suppose two conductors ro meters long are ro cm apart, then by (99)

$$
\begin{aligned}
M & =2000\left[\log _{e} \frac{2000}{\mathrm{IO}}-\mathrm{I}+\frac{\mathrm{IO}}{\mathrm{IOOO}}\right] \\
& =2000[5.2983-0.9900] \\
& =86 \mathrm{I} 6.6 \mathrm{~cm}=8.6 \mathrm{I} 66 \text { microhenrys. }
\end{aligned}
$$


The formula (98) gives a value less than two parts in one hundred thousand greater.

EXAMPLE 72. FORMULAS (100) AND (101)

Suppose a return circuit of two parallel wires, each ro meters long and $0.2 \mathrm{~cm}$ diameter, distant apart $10 \mathrm{~cm}$, center to center, Fig. 49. The self-inductance of the circuit, neglecting the ends, is by (IOO)

$$
\begin{aligned}
L & =4000\left[\log _{e} \frac{\mathrm{IO}}{\mathrm{O} . \mathrm{I}}+\frac{\mathrm{I}}{4}-\frac{\mathrm{IO}}{\mathrm{IOOO}}\right] \\
& =4000 \times 4.845^{2} \\
& =\mathrm{I} 9380.8 \mathrm{~cm}=\mathrm{I} 9.3808 \text { microhenrys. }
\end{aligned}
$$

We have already calculated (example 70) the selfinductance of one of these two wires by itself. Doubling the value we have 36.6140 microhenrys as the selfinductance of two wires in series. In example $7 \mathrm{I}$ we calculated the mutual inductance of these two wires. Doubling the value for $M$ we have I 7.2332 microhenrys. The resultant self-inductance of the circuit (neglecting the ends) is

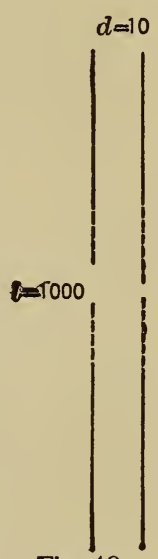

Fig. 49

$$
\begin{aligned}
L=2 L_{1}-2 M & =36.6140-\mathrm{I} 7.2332 \\
& =19.3808 \text { microhenrys. }
\end{aligned}
$$

as found above by formula (IOo).

Taking account of the ends neglected above, we should find that $2 L_{1}$ for the two ends by (95) is $18 \mathrm{I} .9 \mathrm{~cm}$ and $2 M$ by (98) is practically zero. Hence the self-inductance of the circuit is, including the ends,

$$
L=\mathrm{I} 9.5627 \text { microhenrys. }
$$

EXAMPLE 73. FORMULA (102) FOR THE MUTUAL INDUCTANCE OF ADJACENT CONDUCTORS IN THE SAME STRAIGHT LINE

When the two conductors are of equal length, $l=m$, and (102) becomes

$$
M=2 l \log _{e} 2=2 l \times 0.69315 \mathrm{~cm} .
$$

If $l=1000 \mathrm{~cm}, M=\mathrm{I} 386.3 \mathrm{~cm}$. 
If $m=1000 l,(8 \mathrm{I})$ gives

$$
\begin{aligned}
M & =l \log _{e} \text { IOOI }+ \text { IOOO } l \log \mathrm{I} .00 \mathrm{I} \\
& =l \log _{e} \text { IOOI }+l \text { approximately. }
\end{aligned}
$$

If $l=\mathrm{I} \mathrm{cm}$, we have

$$
\begin{aligned}
M & =\log _{e} \text { IOOI }+ \text { IOOO } \log _{e} \text { I.OOI } \\
& =6.909+0.999=7.908 \mathrm{~cm} .
\end{aligned}
$$

The self-inductance of the short wire $\mathrm{AB}$, supposed I $\mathrm{cm}$ long and of $\mathrm{I} \mathrm{mm}$ radius, is

$$
L=2\left(\log _{e} \frac{2}{\mathrm{O} . \mathrm{I}}-.75\right)=2(2.9957-.75)=4.49 \mathrm{I} 5 \mathrm{~cm},
$$

which is a little more than one-half of the mutual inductance of $A B$ and $\mathrm{BC}, \mathrm{BC}$ being one thousand times the length of $\mathrm{AB}$.

In closed circuits, all the magnetic lines due to a circuit are effective in producing self-inductance, and hence the self-inductance is always greater than the mutual inductance of that circuit with any other, assuming one turn in each. But with open circuits, as in this case, we may have a mutual inductance between two single conductors greater than the self-inductance of one of them.

EXAMPLE 74. FORMULA (104) FOR THE SELF-INDUCTANCE OF A RECTANGULAR BAR

In formula (Iò4), substituting $l=1000$, and $\alpha+\beta=2$ for a square bar $1000 \mathrm{~cm}$ long and I square $\mathrm{cm}$ section, we have, neglecting the small last term,

$$
\begin{aligned}
L & =2000\left[\log _{e} \frac{2000}{2}+\frac{1}{2}\right] \\
& =2000(6.908+0.5)=148 \mathrm{I} 6 \mathrm{~cm} \\
& =14.8 \mathrm{I} 6 \text { microhenrys. }
\end{aligned}
$$

This would also be the self-inductance for any section having $\alpha+\beta=2 \mathrm{~cm}$.

EXAMPLE 75. FORMULAS (105) AND (106) FOR THE SELF-INDUCTANCE OF A SQUARE MADE UP OF A ROUND WIRE

If the side of the square is I meter, $a=100 \mathrm{~cm}, \rho=0.1 \mathrm{~cm}$, we have from (105)

$$
\begin{aligned}
L & =800\left(\log _{e} \text { I000 }-0.524\right) \\
& =5107 \mathrm{~cm}=5.107 \text { microhenrys. }
\end{aligned}
$$


If $\rho=.05 \mathrm{~cm}$,

$$
L=5662 \mathrm{~cm}=5.662 \text { microhenrys. }
$$

That is, the self-inductance of such a rectangle of round wire is about I I per cent greater for a wire I $\mathrm{mm}$ in diameter than for one $2 \mathrm{~mm}$ in diameter.

If $l / \rho$ is constant, $L$ is proportional to $l$, that is, if the thickness of the wire is proportional to the length of the wire in the square, the self-inductance of the square is proportional to its linear dimensions.

\section{EXAMPLE 76. FORMULA (107) FOR THE SELF-INDUCTANCE OF A RECTANGLE OF ROUND WIRE}

Suppose a rectangle 2 meters long and I meter broad.

Substituting $a=200 \mathrm{~cm}, b=100, \rho=0.1$, in (107) we have

$$
L=8 \text { oI } 7.1 \mathrm{~cm}=8.017 \text { microhenrys. }
$$

We can obtain the same result from the values of self and mutual inductances calculated in examples 70 and $7 \mathrm{r}$. That is, the resultant self-inductance of the rectangle is the sum of the self-inductances of the four sides, minus twice the mutual inductances of the two pairs of opposite sides. Thus

$$
L=\left(L_{1}+L_{3}\right)+\left(L_{2}+L_{4}\right)-2 M_{13}-2 M_{24}
$$

By example $70, L_{1}+L_{3}=6035.24$

$$
L_{2}+L_{4}=2740.36 \quad 8775.60
$$

By example 7I, $2 M_{13}=\overline{660.48}$

$$
\begin{aligned}
2 M_{24}=\frac{98.04}{\therefore L} & =\frac{75^{8.52}}{8017.08 \mathrm{~cm}} \\
& =8.017 \mathrm{I} \text { microhenrys. }
\end{aligned}
$$

The agreement of this result with that obtained from formula (I07) serves as a check on the latter formula, and also illustrates how the values of the self and mutual inductances of open circuits may be combined to give the self-inductance of a closed circuit. 
EXAMPLE 77. FORMULAS (108), (109), AND (110) FOR THE SELF-INDUCTANCE OF A RECTANGLE OR SQUARE MADE UP OF A BAR OF RECTANGULAR SECTION

$$
\text { Let } a=200 \quad b=100 \quad a=\beta=1.0 \mathrm{~cm} \text {. }
$$

Substituting these values in (IO8) we obtain

$$
\begin{aligned}
L & =4(297 \text { I.05- I } 209.76-577.95-\text { I } 50+447.2 \mathrm{I}+0.99) \\
& =5926.16 \mathrm{~cm} .
\end{aligned}
$$

For a square Io meters on a side, made of square bar I sq $\mathrm{cm}$ cross section we have $a=\mathrm{IOOO}, a=\mathrm{I}$; substituting in (I IO)

$$
\begin{aligned}
L & =8000(6.908+.033) \\
& =8000 \times 6.94 \mathrm{I} \mathrm{cm}=55.53 \text { microhenrys. }
\end{aligned}
$$

For a circular section, diameter I $\mathrm{cm}, \rho=0.5$; substituting in (IO5)

$$
\begin{aligned}
L & =8000\left(\log _{e} 2000+\frac{\mathrm{I}}{2000}-0.524\right) \\
& =8000 \times 7.076 \mathrm{~cm}=56.6 \mathrm{I} \text { microhenrys }
\end{aligned}
$$

a little more than for a square section, as would be expected.

EXAMPLE 78. FORMULA (112) FOR THE MUTUAL INDUCTANCE OF PARALLEL SQUARES

Suppose two parallel squares each I meter on a side, Io $\mathrm{cm}$ distant from one another.

$$
\begin{aligned}
a & =\mathrm{IOO}, d=\mathrm{IO} . \quad \text { Substituting in (II }), \\
M & =8\left[\mathrm{IOO} \log _{e}\left(\frac{\mathrm{I}+\sqrt{\mathrm{I} . \mathrm{OI}}}{\mathrm{I}+\sqrt{2 . \mathrm{II}}} \cdot \frac{\sqrt{\mathrm{IOI}}}{\mathrm{I}}\right)+\sqrt{2 \mathrm{OIOO}}-2 \sqrt{\mathrm{IOIOO}}+\mathrm{IO}\right] \\
& =800\left[\log _{e}\left(\frac{\mathrm{IO} . \mathrm{I}+\sqrt{\mathrm{IOI}}}{\mathrm{I}+\sqrt{2 . \mathrm{OI}}}\right)+\sqrt{2 . \mathrm{OI}}-2 \sqrt{\mathrm{I} . \mathrm{OI}}+\mathrm{O} . \mathrm{I}\right] \\
& =\mathrm{II} 42.5 \mathrm{~cm}=\mathrm{I} . \mathrm{I} 425 \text { microhenrys. }
\end{aligned}
$$

EXAMPLE 79. FORMULAS (113), (114), AND (115) FOR THE SELF AND MUTUAL INDUCTANCE OF THIN STRAIGHT STRIPS OR TAPES

Let the tape of thin copper be Io meters long and I $\mathrm{cm}$ wide. 
Substituting $l=$ IOOO and $b=\mathrm{I}$ in (II 3 ) we have

$$
\begin{aligned}
L=2000\left(\log _{e} 2000+\frac{\mathrm{I}}{2}\right) \\
=2000 \times 8.1009=\mathrm{I} 6202 \mathrm{~cm} \\
=\mathrm{I} 6.202 \text { microhenrys }
\end{aligned}
$$

as the self-inductance when the conducting strip is very thin. If the tape is $2 \mathrm{~mm}$ thick we may allow for the effect of the thickness by using (II4) and we find

$$
L=2000 \times 7.9009 \mathrm{~cm}=\mathrm{I} 5.802 \text { microhenrys, }
$$

which differs slightly from the preceding value.

Two such tapes edge to edge in one plane will have a mutual inductance by (II 5 ) of

$$
\begin{aligned}
M & =2000\left(\log _{e} 2000-0.8863\right) \\
& =2000 \times 6.7 \mathrm{I} 46 \mathrm{~cm} \\
& =\mathrm{I} 3.429 \text { microhenrys. }
\end{aligned}
$$

EXAMPLE 80. FORMULA (117) FOR THE SELF-INDUCTANCE OF A RETURN CIRCUIT OF TWO PARALLEL SHEETS; NONINDUCTIVE SHUNTS

Suppose the dimensions of a thin manganin sheet which has been doubled on itself be as follows:

$$
l=30 \mathrm{~cm} \quad b=10 \mathrm{~cm} \quad d=\mathrm{I} \mathrm{cm} .
$$

By (I 32) $\log R_{2}=$ I.0787

$$
\begin{aligned}
\log R_{1} & =\log _{e} \mathrm{IO}-\frac{3}{2}=0.8026 \\
L & =4 l\left(\log R_{2}-\log R_{1}\right) \\
& =\mathrm{I} 20 \times 0.276 \mathrm{I} \\
& =33 . \mathrm{I} 3 \mathrm{~cm} \\
& =.033 \mathrm{I} \text { microhenrys. }
\end{aligned}
$$

EXAMPLE 81. FORMULA (122), 3 CONDUCTORS IN MULTIPLE

Suppose three cylindrical conductors, each ro meters long and $4 \mathrm{~mm}$ diameter, the distance apart of their centers being $\mathrm{I} \mathrm{cm}$. Substitute in (122) as follows:

$$
l=1000 \mathrm{~cm} \quad \rho=2 \mathrm{~mm} \quad d=\mathrm{I} \mathrm{cm} .
$$


Then $\quad\left(r_{g} a^{2}\right)^{\frac{1}{3}}=0.53^{8} \mathrm{~cm}$

and

$$
\begin{aligned}
L & =2000\left(\log _{e} \frac{2000}{0.53^{8}}-\mathrm{I}\right) \\
& =2000 \times 7.22 \mathrm{Im}=\mathrm{I} 4.442 \text { microhenrys. }
\end{aligned}
$$

If the whole current flowed through a single one of the three conductors the self-inductance would be

$$
L=2000\left(\log _{e} \frac{2000}{0.2}-\frac{3}{4}\right)=x 7.92 \text { microhenrys, }
$$

or about 25 per cent more than when divided among the three.

\section{FORMULAS FOR GEOMETRICAL AND ARITHMETICAL MEAN DISTANCES}

\section{GEOMETRICAL MEAN DISTANCES}

Maxwell showed how to calculate mutual and self-inductances in several important cases by means of what he called the geometrical mean distance, either of one conductor from another or of a conductor from itself. On account of the importance of this method we give below some of the most useful of these formulas. The geometrical mean distance of a point from a line is the $n^{\text {th }}$ root of

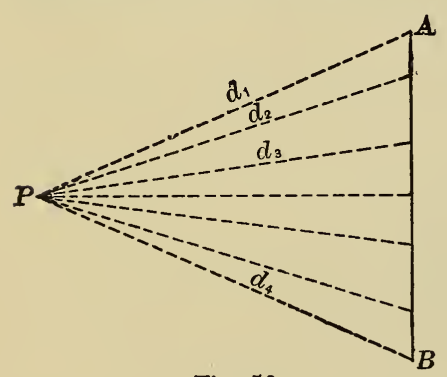

Fig. 50

the product of the $n$ distances from the point $P$ to the various points in the line, $n$ being increased to infinity in determining the value of $R$. Or, the logarithm of $R$ is the mean value of log $d$ for all the infinite values of the distance $d$. Similarly, the geometrical mean distance of a line from itself is the $n^{\text {th }}$ root of the product of the $n$ distances between all the various pairs of points in the line, $n$ being infinity. ${ }^{109}$

Similar definitions apply to the $g . m$. d. of one area from another, or of an area from itself. 
The geometrical mean distance $R$ of a line of length $a$ from itself is given by

$$
\begin{aligned}
\log R & =\log a-\frac{3}{2} \\
R & =a e^{-\frac{3}{2}} \\
\text { or } R & =0.223 \text { I } 3 a
\end{aligned}
$$

The g. m. d. of a rectangular area of sides $a$ and $b$ from itself is given by

$$
\begin{gathered}
\log R=\log \sqrt{a^{2}+b^{2}}-\frac{\mathrm{I}}{6} \frac{a^{2}}{b^{2}} \log \sqrt{\mathrm{I}+\frac{b^{2}}{a^{2}}}-\frac{\mathrm{I}}{6} \frac{b^{2}}{a^{2}} \log \sqrt{\mathrm{I}+\frac{a^{2}}{b^{2}}} \\
+\frac{2}{3} \frac{a}{b} \tan ^{-1} \frac{b}{a}+\frac{2}{3} \frac{b}{a} \tan ^{-1} \frac{a}{b}-\frac{25}{\mathrm{I} 2}
\end{gathered}
$$

When the area is a square, and hence $a=b$,

$$
\begin{aligned}
& \log R=\log a+\frac{\mathrm{I}}{3} \log 2+\frac{\pi}{3}-\frac{25}{\mathrm{I} 2} \\
& \therefore R=0.44705 a
\end{aligned}
$$

For a circular area of radius $a$,

$$
\begin{aligned}
\log R & =\log a-\frac{I}{4} \\
R & =a e^{-\frac{1}{4}} \\
R & =0.7788 a
\end{aligned}
$$

For an ellipse of semi-axes $a$ and $b$,

$$
\log R=\log \frac{a+b}{2}-\frac{\mathrm{I}}{4}
$$

An approximate expression for the g. $m$. d. of a rectangular area of length $a$ and breadth $b$ is

$$
R=0.2235(a+b)
$$

wh1ch is nearly true for all values of $a$ and $b$; that is, the geometrical mean distance of the rectangular area from itself is approximately proportional to the perimeter of the rectangle. The following table gives the ratio $R /(a+b)$ for a series of rectangles of different proportions, from a square to a ratio of 20 to $\mathrm{I}$ between length and breadth, and finally when the breadth is infinitesimal in comparison with the length. By interpolating for any other case between the 
values given in the table one can obtain a quite accurate value without the trouble of calculating it by formula (I24).

Geometrical Mean Distances of Rectangles of Different Proportions

[ $a$ and $b$ are the Length and Breadth of the Rectangles. $R$ is the Geometrical Mean Distance of its Area]

\begin{tabular}{cc|c|c}
\hline \multicolumn{2}{c|}{ Ratio } & $R$ & $\frac{R}{a+b}$ \\
\hline 1 & $: 1$ & $0.44705 a$ & 0.22353 \\
$1.25: 1$ & $0.40235 a$ & 0.22353 \\
1.5 & $: 1$ & $0.37258 a$ & 0.22355 \\
2 & $: 1$ & $0.33540 a$ & 0.22360 \\
4 & $: 1$ & $0.27961 a$ & 0.22369 \\
10 & $: 1$ & $0.24596 a$ & 0.22360 \\
20 & $: 1$ & $0.23463 a$ & 0.22346 \\
1 & $: 0$ & $0.22315 a$ & 0.22315 \\
\hline
\end{tabular}

The g. m. d. of an annular area of radii $a_{1}$ and $a_{2}$ from itself is given by

$$
\log R=\log a_{1}-\frac{a_{2}^{4}}{\left(a_{1}^{2}-a_{2}^{2}\right)^{2}} \log \frac{a_{1}}{a_{2}}+\frac{\mathrm{I}}{4} \frac{3 a_{2}^{2}-a_{1}^{2}}{a_{1}^{2}-a_{2}^{2}}
$$

The g. m. d. of a line of length $a$ from a second line of the same length, distant in the same straight line na, - $a$ center to center, Fig. 5I, is given by the following formula:

Fig. 51

$\log R_{n}=\frac{(n+\mathrm{I})^{2}}{2} \log (n+\mathrm{I}) a-n^{2} \log n a+\frac{(n-\mathrm{I})^{2}}{2} \log (n-\mathrm{r}) a-\frac{3}{2}$

This formula is equivalent to the following, which is more convenient for calculation for all values of $n$ greater than one. ${ }^{110}$

$\log R_{n}=\log n-\left[\frac{\mathrm{I}}{\mathrm{I} 2 n^{2}}+\frac{\mathrm{I}}{60 n^{4}}+\frac{\mathrm{I}}{\mathrm{I} 68 n^{6}}+\frac{\mathrm{I}}{360 n^{8}}+\frac{\mathrm{I}}{660 n^{10}}+\cdots\right]_{[\mathrm{I} 3 \mathrm{I}]}$

This formula is very convergent, and only two or three terms are generally required. 
The following values of the geometrical mean distances (calling $a$ unity) were calculated from the above formulas, all after the second being obtained by ( $13 \mathrm{I}$ ):

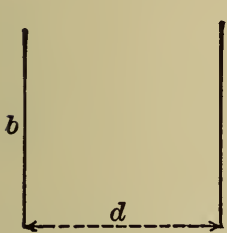

Fig. 52

$\begin{array}{ll}R_{0}=0.223 \mathrm{I} 3 & R_{5}=4.98323 \\ R_{1}=0.8925^{2} & R_{6}=5.986 \mathrm{IO} \\ R_{2}=\mathrm{I} .95653 & R_{7}=6.98806 \\ R_{3}=2.97 \mathrm{I} 7 \mathrm{I} & R_{8}=7.98957 \\ R_{4}=3.97890 & R_{9}=8.99076\end{array}$

If the lines are parallel and at distance $d$, Fig. 52 , the $\mathrm{g} . \mathrm{m}$. $\mathrm{d}$. is given by

$\log R=\frac{d^{2}}{b^{2}} \log d+\frac{\mathrm{I}}{2}\left(\mathrm{I}-\frac{d^{2}}{b^{2}}\right) \log \left(b^{2}+d^{2}\right)+2 \frac{d}{b} \tan ^{-1} \frac{b}{d}-\frac{3}{2}$

'If $d=b$,

$$
\log R=\log b+\frac{\pi}{2}-\frac{3}{2}
$$

The g. m. d. from a point $\mathrm{O}_{2}$, Fig. 53 , outside a circle to the circumference of the circle, or to the entire area of the circle $i s$ the distance $d$ from $\mathrm{O}_{2}$ to the center of the circle.

(I) The g. m. d. from the center $\mathrm{O}_{1}$ to the circumference is of

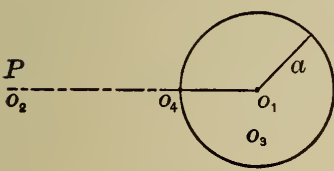

Fig. 53 course the radius $a$. (2) The g. m. d. of any point $\left(\right.$ as $\left.\mathrm{O}_{3}\right)$ within the circle from the circumference is also $a$. (3) The g.m.d. of any point on the circumference (as $\mathrm{O}_{4}$ ) from all other points of the circumference is also $a$.

(4) Therefore the g. m. d. of a circular line of radius $a$ from itself is $a$; that is,

$$
R=a
$$

for each of the four cases named above.

The g. m. d. of a point outside a circular ring, Fig. 54, from the ring is the distance $d$ to the center of the ring. The g. m. d. of any point $\mathrm{O}_{1}, \mathrm{O}_{3}$, etc., within the ring is given by

$$
\log R=\frac{a_{1}{ }^{2} \log a_{1}-a_{2}{ }^{2} \log a_{2}}{a_{1}{ }^{2}-a_{2}{ }^{2}}-\frac{\mathbf{I}}{2}
$$

The same expression gives the g. m. d. of any figure, as $S_{1}$, within the ring from the ring. The $\mathrm{g}$. m. d. of an external figure,

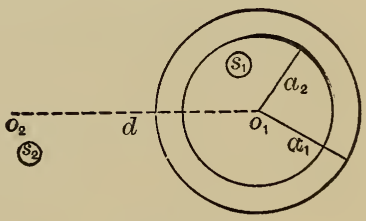

Fig. 54 
as $\mathrm{S}_{2}$, from the annular ring is equal to the g. m. $\mathrm{d}$. of the center $\mathrm{O}_{1}$ from the figure $S_{2}$.

The g. m. d. from one circular area to another is the distance between their centers; that is,

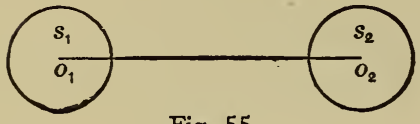

Fig. 55

$$
R=d
$$

for the area $S_{1}$ with respect to $S_{2}$ as it is for the point $O_{1}$ with respect to $S_{2}$.

The g. m. d. of a line of length $a$ from a second parallel line of length $a^{\prime}$ located symmetrically (Fig. $5^{6}$ ) is given by Gray ${ }^{111}$, equation (I I4). The g. m. d. of a line from a parallel and symmetrically

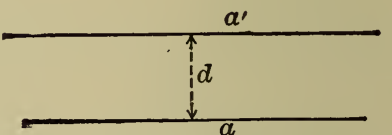

Fig. 56 situated rectangle is given by Gray's equation (I I 2). The g. m. d. of two unequal rectangles from one another is given by Gray's equation (II 3$) \cdot{ }^{112}$

The g. m. d. of two adjacent rectangles and of two obliquely situated rectangles are given by Rosa, ${ }^{113}$ equations ( $8 a$ ) and (I 7). As these expressions are somewhat lengthy and not often required they are not repeated here. The values of the g. m. d. for two equal squares in various relative positions to one another have been accurately calculated ${ }^{114}$ by these formulas, and the results used in the determination ${ }^{115}$ of the correction term $E$ of formula (93).

${ }^{111}$ Absolute Measurements, Vol. II, Part I.

There are a number of misprints in equations I04, IO9, III, and II 3 of Gray. The sign of the first term of equation ro 4 should be + . The signs before $p^{2}$ in the coefficients of the $\log$ in the first four terms of equation Iog should be all minus; thus $1 / 4$ $\left(\beta^{2}-p^{2}\right),-1 / 4\left(\alpha^{2}-p^{2}\right),-1 / 4\left[(\alpha-\beta)^{2}-p^{2}\right],+1 / 4\left[(a-\alpha)^{2}-p^{2}\right]$. Similarly in equation III the coefficients of the first two terms should be $1 / 2\left(\beta^{2}-p^{2}\right)$ and $-1 / 2\left(\alpha^{2}-p^{2}\right)$. In equation 113 the coefficient of $\beta^{4}$ in each of the first four terms should be $1 / 6$ instead of $1 / 2$ and the first term should have $\log \left[\left(p+b+b^{\prime}\right)^{2}+\beta^{2}\right]$ instead of $\log$ $\left[\left(p+b+b^{\prime}\right)^{2}-\beta^{2}\right]$.

${ }^{112}$ Also by Rosa, equation (8) this Bulletin, 3, p. 6; 1907.

${ }^{113}$ This Bulletin, 3, pp. 7 and 12 ; 1907.

${ }^{114}$ This Bulletin, 3, pp. 9-19; 1907.

${ }^{115}$ This Bulletin, 3, p. 37; r907. 


\section{ARITHMETICAL MEAN DISTANCES}

In the determination of self and mutual inductances by the method of geometrical mean distances it has been shown ${ }^{116}$ that more accurate formulas can be obtained by the use of certain arithmetical mean distances and arithmetical mean square distances taken in connection with geometrical mean distances.

The arithmetical mean distance of a point from a line is the arithmetical mean of the $n$ distances of the point from the various points of the line, $n$ being infinite. Similarly, the arithmetical mean distance of a line from itself is the arithmetical mean of the distances of the $n$ pairs of points in the line from one another, $n$ being infinite.

The a. m. d. of a line of length $b$ from itself is ${ }^{117}$

$$
S_{2}=\frac{b}{3}
$$

that is, while the g. m. d. of a line from itself is $0.223 \mathrm{I} 3$ times its length, the a. m. d. is one-third the length.

The arithmetical mean square distance of a line from itself is of course larger than the square of the a. m. d. Putting $S_{2}{ }^{2}$ for the arithmetical mean square distance (a. m. s. d.).

$$
S_{2}^{2}=\frac{b^{2}}{6} \text { or } \sqrt{S_{2}^{2}}=\frac{b}{\sqrt{6}}
$$

The arithmetical mean distance of a point in the circumference of a circle from the circle is the same as the a. $\mathrm{m}$. $\mathrm{d}$. of the circle from itself; that is, for a circle of radius $a$,

$$
S_{1}=S_{2}=\frac{4}{\pi} a
$$

The arithmetical mean square distance is

$$
S_{2}^{2}=2 a^{2} \text { and } \sqrt{S_{2}^{2}}=a \sqrt{2}
$$

(The g. m. d. for this case is $R=a$, equation (I34).) 
The arithmetical mean distance of an external point $\mathrm{P}$ from the circumference of a circle, Fig. 57, is

$$
S_{1}=\sqrt{d^{2}+a^{2}}
$$

which is the distance PA.

The arithmetical mean distance from $P$ to the entire area of the circle is

$$
S_{1}=\sqrt{d^{2}+\frac{a^{2}}{2}}
$$

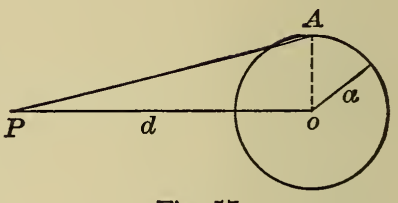

Fig. 57

(The g. m. d. for each of these cases is $R=d$, equation (I 36 ).)

For the proof of these and other expressions for the arithmetical mean distances and applications of their use see the article referred to above.

\section{HIGH-FREQUENCY FORMULAS}

Excepting in a very few specified cases, the formulas of the preceding sections apply only to conductors carrying direct current or alternating currents of frequencies so low that the error, due to the assumption that the current is uniformly distributed over the cross section of the wire, is negligible.

In the case of standards of mutual inductance the inductance may be regarded as sensibly independent of the frequency, unless the two coils are very close together, and even then the capacity between the coils will be a more potent source of error than the departure of the current from a uniform distribution over the cross section of the wire.

The self-inductance of a coil or conductor, on the other hand, depends appreciably on the field in the cross section of the conductor, and any deviation of the distribution of the current in the wire from uniformity gives rise to a decrease in the inductance. The amount of this change depends on the frequency of the current and the radius of the cross section of the conductor, as well as on the conductivity and permeability of the material of which it is composed.

This decrease of the inductance is accompanied by an increase in the resistance of the conductor. Whereas, however, the inductance 
with increasing frequency approaches a limiting value, the resistance increases indefinitely as the frequency approaches an infinite value. The change of resistance is always relatively much larger than the change in inductance.

The eddy current effects just described are, for the most part, negligible at low frequencies, except in the case of heavy conductors and in coils wound with stout wire in several layers. In the latter case, however, the diminution of the inductance, due to the irregular distribution of the current, is marked, to a greater or less degree, by the effect of the capacity between the windings of the coil, which gives rise to an increase of the inductance with the frequency. For the same reason the resistance is increased more than it would be by the eddy currents alone.

Unfortunately, the rigorous or approximate solution of the problem at high frequencies for the various cases for which the inductance with steady currents may be calculated is in many instances very difficult, if not impossible. Some of the simpler cases, however, because of their great importance, have received much attention, with the result that the changes of inductance and resistance may be calculated with a good degree of precision.

\section{STRAIGHT CYLINDRICAL WIRES}

This is the most important case of all, since the solution is rigorous, and the results may be applied to the construction of practical, absolute standards for high-frequency work. The problem has been treated successively by Maxwell, ${ }^{118}$ Heaviside, ${ }^{119}$ Rayleigh, ${ }^{120}$ and Kelvin. ${ }^{21}$

Putting $l=$ length of conductor

$\rho=$ radius of conductor

$\sigma=$ specific resistance of its material

$\mu=$ permeability

$f=$ frequency, $p=2 \pi f$

$R^{\prime}=$ resistance with current of frequency $f$

$L^{\prime}=$ inductance " " " " $f$

118 Elect. and Mag., II, \& 690.

119 Elect. Papers, II, p. 64 .

${ }^{120}$ Phil. Mag., 21, p. 38I; 1886.

121 Math. and Phys. Papers, III, p. 49I; 1889. 
$R=$ resistance with direct current

$L=$ inductance " " "

$$
x=2 \rho \sqrt{\frac{\pi p \mu}{\sigma}}
$$

Thus

$$
\begin{aligned}
& \frac{R^{\prime}}{R}=\frac{x}{2} \frac{W}{Y} \\
& L^{\prime}=2 l\left[\log \frac{2 l}{\rho}-\mathrm{I}+\frac{\mu}{4}\left(\frac{4}{x} \cdot \frac{Z}{Y}\right)\right]
\end{aligned}
$$

where

$$
\begin{aligned}
W & =\text { ber } x \text { bei }^{\prime} x \text {-bei } x \text { ber }^{\prime} x \\
Y & =\left(\text { ber }^{\prime} x\right)^{2}+\left(\text { bei }^{\prime} x\right)^{2} \\
Z & =\operatorname{ber} x \text { ber }^{\prime} x+\text { bei } x \text { bei }^{\prime} x
\end{aligned}
$$

Since from $(96)$

$$
L=2 l\left[\log \frac{2 l}{\rho}-\mathrm{I}+\frac{\mu}{4}\right]
$$

we find

$$
\begin{aligned}
& \Delta L=L^{\prime}-L=-2 l \frac{\mu}{4}\left[\mathrm{I}-\frac{4}{x} \frac{Z}{Y}\right] \\
& \frac{\Delta L}{L}=-\frac{\frac{\mu}{4}\left(\mathrm{I}-\frac{4}{x} \frac{Z}{Y}\right)}{\log \frac{2 l}{\rho}-\mathrm{I}+\frac{\mu}{4}}
\end{aligned}
$$

For nonmagnetic material the equation ( 146 ) takes the form

$$
\frac{\Delta L}{L}=-\frac{\left(\mathrm{I}-\frac{4}{x} \frac{Z}{Y}\right)}{4 \log \frac{2 l}{\rho}-3}
$$

In these expressions, ber $x$ and bei $x$ are functions introduced by Lord Kelvin, being respectively the real and imaginary parts of the ordinary Bessel function of order zero, $J_{0}$, having for its argument $x i \sqrt{i}$, where $x$ is a real quantity, and $i=\sqrt{-\mathrm{I}}$. These functions are given by the series 


$$
\left.\begin{array}{l}
\text { ber } x=\mathrm{I}-\frac{x^{4}}{2^{2} 4^{2}}+\frac{x^{8}}{2^{2} 4^{2} 6^{2} 8^{2}}-\ldots \ldots \\
\text { bei } x=\frac{x^{2}}{2^{2}}-\frac{x^{6}}{2^{2} 4^{2} 6^{2}}+\frac{x^{10}}{2^{2} 4^{2} 6^{2} 8^{2} \mathrm{IO}^{2}}-\ldots
\end{array}\right\}
$$

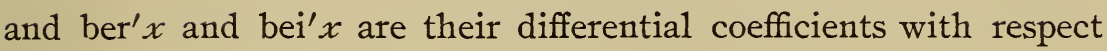
to $x$.

These series are very convergent, but the calculation, naturally, becomes laborious for large values of $x$. To lighten the labor of calculation Russel1 ${ }^{122}$ and Savidge ${ }^{123}$ have developed asymptotic expressions for ber $x$, bei $x$, ber ' $x$, bei ' $x$ and the auxiliary quantities $W, Y$, and $Z$, which give their numerical values with an accuracy of about one part in ten thousand for values of $x$ greater than about 6 , but whose accuracy increases rapidly as $x$ becomes larger.

Savidge ${ }^{123}$ has, in addition, calculated extensive tables of the above functions and the allied ker and kei functions to four places of decimals, and for values of the argument ranging between $I$ and 30 in steps of one unit. These tables will be found very useful in the solution of a variety of problems. For calculation with the formulas (I43) to (I47), however, it seemed desirable to construct tables in which the argument advances by smaller steps than in the tables of Savidge. For this purpose ber $x$, bei $x$, ber ' $x$ and bei ' $x$ were calculated directly from their series, for arguments from o.I to 5.o, in steps of 0.1 , and from 5 to 7 in steps of 0.2. From these were obtained directly by (144a) the values of $W, Y, Z$. For the larger values of $x$, the quantities $W, Y, Z$ were calculated by asymptotic formulas, and checked at a few points by the direct series. Thus the interval from 5 to ro was covered in steps of 0.2 , the interval Io to 15 in steps of 0.5 , and from 15 to 50 in steps of one unit, the aim being to keep the differences of the same order of magnitude. It will, probably, seldom be necessary to make calculations for values of $x$ greater than about 5 o. If such calculations are occasionally required, they may be made with little trouble by the asymptotic formulas given below. 
Since making the above calculations, we have determined the expressions for the general terms in Russell's equations (8), (9), and (Io), (loc. cit., p. 529), thus materially increasing their range of applicability.

These equations, thus extended, are

$$
\begin{aligned}
& W=\frac{x}{2}\left\{\mathrm{I}+\frac{6}{(\mid \underline{3})^{2}}\left(\frac{x}{2}\right)^{4}+\frac{30}{(\underline{5})^{2}}\left(\frac{x}{2}\right)^{8}+\frac{\mathrm{I} 4 \mathrm{O}}{(\underline{7})^{2}}\left(\frac{x}{2}\right)^{12}+\cdots \cdot\right. \\
& \left.+\frac{\mathrm{I} \cdot 2 \cdot 3 \cdot \cdots \cdot(2 n+\mathrm{I})}{\mathrm{I}^{2} 2^{2} 3^{2} \cdot \cdots \cdot n^{2}(\underline{\mid 2 n+\mathrm{I}})^{2}}\left(\frac{x}{2}\right)^{4 n}+\cdots\right\} \\
& Y=\frac{x^{2}}{4}\left\{\mathrm{I}+\frac{3}{(\mid \underline{3})^{2}}\left(\frac{x}{2}\right)^{4}+\frac{\text { 10 }}{(\underline{5})^{2}}\left(\frac{x}{2}\right)^{8}+\frac{35}{(\mid \underline{7})^{2}}\left(\frac{x}{2}\right)^{12}+\right. \\
& \left.+\frac{I}{(n+I)} \frac{I \cdot 2 \cdot 3 \cdots \cdots(2 n+I)}{1^{2} 2^{2} 3^{2} \cdots n^{2}\left(\underline{\mid 2 n+I)^{2}}\right.}\left(\frac{x}{2}\right)^{4 n}+\cdots\right\} \\
& Z=\frac{x^{3}}{\mathrm{I} 6}\left\{\mathrm{I}+\frac{3}{2(\mid \underline{3})^{2}}\left(\frac{x}{2}\right)^{4}+\frac{\mathrm{IO}}{3(\mid \underline{5})^{2}}\left(\frac{x}{2}\right)^{8}+\frac{35}{4(\mid \underline{7})^{2}}\left(\frac{x}{2}\right)^{12}+\cdots\right. \\
& \left.\left.+\frac{\mathrm{I}}{(n+\mathrm{I})^{2}} \frac{\mathrm{I} \cdot 2 \cdot 3 \cdots \cdots(2 n+\mathrm{I})}{\mathrm{I}^{2} 2^{2} 3^{2} \cdots n^{2}(\underline{2 n+\mathrm{I}})^{2}}\left(\frac{x}{2}\right)^{4 n}+\cdots\right\}\right)
\end{aligned}
$$

It would have been simpler to have calculated the values of $W, Y$, and $Z$ by these formulas than by the more indirect process actually used. The formulas (I49) have, however, shown themselves of great service in checking the results. For completeness the asymptotic formulas of Savidge used have also been appended. They give results to one in one hundred thousand for $x \geqq$ Io and may be used with an error of not greater than one in ten thousand down to $x=6$.

$$
\left.\begin{array}{rl}
W & =\frac{e^{x \sqrt{2}}}{2 \pi x}\left[\frac{\mathrm{I}}{\sqrt{2}}+\frac{\mathrm{I}}{8 x}+\frac{9}{(8 x)^{2} \sqrt{2}}+\frac{39}{(8 x)^{3}}+\frac{75}{2(8 x)^{4} \sqrt{2}}-\cdots\right] \\
Y & \left.=\frac{e^{x} \sqrt{2}}{2 \pi x}\left[\mathrm{I}-\frac{6}{8 x \sqrt{2}}+\frac{9}{(8 x)^{2}}+\frac{\mathrm{I} 5 \mathrm{O}}{(8 x)^{3} \sqrt{2}}+\frac{2475}{2(8 x)^{4}}+\cdots \cdot\right]\right] \\
Z & =\frac{e^{x} \sqrt{2}}{2 \pi x}\left[\frac{\mathrm{I}}{\sqrt{2}}-\frac{3}{8 x}-\frac{15}{(8 x)^{2} \sqrt{2}}-\frac{45}{(8 x)^{3}}+\frac{3 \mathrm{I} 5}{2(8 x)^{4} \sqrt{2}}+\cdots \cdot\right]
\end{array}\right\}[\mathrm{I} 5 \mathrm{O}]
$$

The results are given in Table XXII which gives the values, to one in one hundred thousand, of not only the quantities $\frac{x}{2} \frac{W}{Y}$ and 
$\frac{4}{x} \frac{Z}{Y}$ required in the preceding formulas, but of $\frac{W}{Y}$ and $\frac{Z}{Y}$ also. These will be found useful in allied problems, and it may seem preferable in some cases to interpolate the values of these latter quantities to obtain the former. For example, with $x>2.5$ the first differences, and in some places the second differences also, are smaller with $\frac{W}{Y}$ than with $\frac{x}{2} \frac{W}{Y}$. The accuracy of the Table XXII may be regarded as greater than will usually be required, and should suffice for the most precise work.

In addition to the general formulas of Kelvin (I43) and (I44), Rayleigh ${ }^{124}$ has given expansions holding for small values of the argument $x$. These equations, which were extended to another term by Heaviside, are, expressed in the present nomenclature,

$$
\left.\begin{array}{l}
\frac{x}{2} \frac{W}{Y}=\mathrm{I}+\frac{\mathrm{I}}{\mathrm{I} 2}\left(\frac{x}{2}\right)^{4}-\frac{\mathrm{I}}{\mathrm{I} 8 \mathrm{o}}\left(\frac{x}{2}\right)^{8}+\frac{\mathrm{II}}{\mathrm{I} 2 \cdot 28 \cdot 30}\left(\frac{x}{2}\right)^{12}-\ldots \\
\frac{4}{x} \frac{Z}{Y}=\mathrm{I}-\frac{\mathrm{I}}{24}\left(\frac{x}{2}\right)^{4}+\frac{\mathrm{I} 3}{4320}\left(\frac{x}{2}\right)^{8}-\frac{647}{\mathrm{I}^{2} \cdot 360 \cdot 56}\left(\frac{x}{2}\right)^{12}+\cdots
\end{array}\right\}
$$

Their applicability is limited to the range of values of $x$ less than about 2, and it will be more convenient to use Table XXII.

For very high frequencies Rayleigh gave also the limiting formulas

$$
\begin{aligned}
& R^{\prime}=\sqrt{\frac{\mathrm{I}}{2} p l \mu R} \\
& L^{\prime}=2 l\left[\log \frac{2 l}{\rho}-\mathrm{I}+\frac{\mathrm{I}}{2} \sqrt{\frac{\mu R}{2 p l}}\right]
\end{aligned}
$$

In some instances these formulas have been used, as though they were exact, over a considerable range of frequencies, without any statement being made as to the magnitude of the error involved. Expressing these formulas in the present nomenclature, we obtain the following formulas for infinite frequencies: 


$$
\begin{aligned}
\left(\frac{R^{\prime}}{R}\right)_{x=\infty} & =\frac{x}{2 \sqrt{2}} \\
\left(L^{\prime}\right)_{x=\infty} & =2 l\left[\log \frac{2 l}{\rho}-\mathrm{I}+\frac{\mu}{4}\left(\frac{4}{x \sqrt{2}}\right)\right]_{x=\infty} \\
& =2 l\left[\log \frac{2 l}{\rho}-\mathrm{I}\right]
\end{aligned}
$$

These are seen to be in agreement with equations (I43) and (I44), if we remember that the limiting values of $\frac{W}{Y}$ and $\frac{Z}{Y}$ as the argument $x$ is indefinitely increased are both $\frac{I}{\sqrt{2}} \cdot$ (See formulas (I 50).) From (I50) we find that only for values of $x$ greater than about 900 is the error from using ( 152 ) as small as one-tenth per cent. For $x=70$ the error is about I per cent, and in many practical cases it is still larger.

The limiting value of the change of inductance is found from (I47) to be

$$
\begin{aligned}
& \left(\frac{\Delta L}{L}\right)_{x=\infty}=-\frac{\mathrm{I}}{4 \log \frac{2 l}{\rho}-3} \\
& (\Delta L)_{x=\infty}=-\frac{l}{2}
\end{aligned}
$$

The error from using ( $\mathrm{I}_{53}$ ) is only about one part in ten thousand for $x=60$. The error, however, arising from the neglect of the term $\frac{4}{x} \frac{Z}{Y}$ in (I 47 ) is more than 5 per cent.

From (I54) we obtain the curious result that the limiting value of the fractional change of inductance, as the frequency is indefinitely increased, depends only on the ratio of the length of the wire to the cross section. Table XXIII gives an idea of the way the limiting value falls off as this ratio is increased.

The preceding formulas show that the change of resistance and inductance are functions of the quantity

$$
x=2 \rho \sqrt{\frac{\pi \mu p}{\sigma}}=2 \pi \rho \sqrt{2 \mu K f}
$$

where $K$ is the conductivity. 
Taking the specific resistance of annealed copper at $20^{\circ}$ as $\mathrm{I} .72 \mathrm{I}$ microhms or I72I in absolute electromagnetic units,

$$
K_{0}=5.8 \mathrm{II} \times \mathrm{IO}^{-4}
$$

and $\left({ }^{6}{ }^{6}\right)$ takes the simple form

$$
x=0.02142 \rho \sqrt{f}
$$

To aid in making approximate calculations, and for purposes of orientation, the auxiliary Table XXIV has been calculated, giving the value $x=x_{0}$ for copper wire of the above conductivity and of a cross section of $\mathrm{I} \mathrm{mm}$ radius at various frequencies. For the higher frequencies, the corresponding wave length $\lambda$ in meters has been included as likely to be of service in calculations for wirelesstelegraph circuits. The range of this table may be considerably extended by remembering that $x$ varies with $\sqrt{f}$ or $\sqrt{\frac{1}{\lambda}}$. Thus the value of $x_{0}$ for 7500 cycles is found directly from the tabulated value for 750 ooo cycles by shifting the decimal point. Similarly, the value for $\lambda=I 50$ meters is obtained from the tabulated value for I 5000 meters. It is for this reason that the larger values of $\lambda$ have been tabulated.

To calculate $x$ for a copper wire of radius $r \mathrm{~mm}$, we have $x=x_{0} r$, and if the conductivity have any value $K$, the further factor $\sqrt{\frac{K}{K_{0}}}$ must be applied. Finally, if the wire is, in addition, of magnetic material of permeability $\mu$, an additional factor $\sqrt{\mu}$ is necessary to obtain the required value of $x$.

\section{CONCENTRIC MAIN}

The simple case of a cylindrical, straight wire may be regarded as a special case of the more general problem of a concentric main; that is, of a solid or tubular inner conductor surrounded by a coaxial tubular outer conductor. This case has been very completely treated by Russell, ${ }^{125}$ but as the formulas are not simple they are not given here. 


\section{TWO PARALLEL WIRES}

Unless the two wires are so near together, relatively to their radius of cross section, that their mutual inductance is appreciably affected by changes in the distribution of the current within the wires, each wire may be treated by the formulas given for a straight, cylindrical wire.

Supposing, therefore, that the wires are alike in every respect

$$
L^{\prime}=4 l\left[\log \frac{d}{\rho}+\frac{\mu}{4}\left(\frac{4}{x} \frac{Z}{Y}\right)\right]
$$

and from (IOI) we find for wires of nonmagnetic material

$$
\begin{aligned}
\frac{\Delta L}{L} & =-\frac{\left(\mathrm{I}-\frac{4}{x} \frac{Z}{Y}\right)}{4 \log \frac{d}{\rho}+\mathrm{I}} \\
\left(\frac{\Delta L}{L}\right)_{x=\infty} & =-\frac{\mathrm{I}}{4 \log \frac{d}{\rho}+\mathrm{I}} \\
(\Delta L)_{x=\infty} & =l \\
\frac{R^{\prime}}{R} & =\frac{x}{2} \frac{W}{Y}
\end{aligned}
$$

the values of $\frac{Z}{Y}$ and $\frac{W}{Y}$ being taken from Table XXII.

Nicholson ${ }^{126}$ has recently given a solution of the problem, when the two wires are so close together that their mutual inductance suffers a sensible change with the frequency. To obtain an idea of the magnitude of this effect, in a practical case, the results by Nicholson's formulas were compared with those of ( $\left.5_{58}\right)$ and ( 160$)$. With $d=I \mathrm{~cm}$ and $\rho=0.1 \mathrm{~cm}$, and with a frequency of $10^{6},\left(15^{8}\right)$ gives $\frac{\Delta L}{L}=-8.5$ per cent, the effect of Nicholson's correction being to give a value of $\frac{\Delta L}{L}$ numerically only nine parts in ten thousand smaller. 
Similarly for the resistance, ( 160 ) gives $\frac{R^{\prime}}{R}=7 \cdot 56$, while Nicholson's formula reduces this to $7 \cdot 55$. Since this example relates to a rather unfarorable case, for a standard whose inductance is to be calculated from the dimensions, these corrections for mutual effect may, in general, be regarded as negligible, and the formulas ( $\mathrm{I}_{5} S$ ), (I59), and (I60) may be regarded as sufficiently accurate with the precision usually attainable in the measurement of the dimensions.

It is to be noticed that the maximum possible relative change of inductance, with the frequency, is greater with two parallel wires than with either alone, because this change with the parallel wires depends on the sum of their self-inductances which is greater than the resulting self-inductance of the combination (see p. I5I). Table XXIII gives an idea of the values attained $b_{y}\left(\frac{\Delta L}{L}\right)_{x=x}$ in the case of the two parallel wires. This maximum change of inductance depends only on the ratio of their distance apart to the radius of cross section of the wire.

Evidently; other cases of linear conductors of circular cross section, may likewise be made to depend on the solution for straight wires.

\section{CIRCULAR RING OF CIRCULAR SECTIOY}

The inductance of a circular ring, in which the current is confined wholly to the circumference of the cross section was given in formula $\left(6_{5}\right)$. Combining this with $\left(6_{3}\right)$ we find that on the assumption that $(65)$ represents the actual distribution of the current at infinite frequency;

$$
(\lrcorner L)_{x=r}=-\pi a\left[I-\frac{I}{2} \rho^{2}\left(\log \frac{S a}{\rho}-\frac{I}{3}\right)\right]
$$

The absolute value of the change of inductance at infinite frequency is, in the case of a straight wire, (see I 45 )

$$
(\lrcorner L)_{x=3 x}=-\frac{i}{2}
$$

which shows that, if the wire of the ring were stretched out straight, the ralue given in (I6I) would become

$$
(\lrcorner L)_{x=x}=-\frac{1}{2} 2 \pi a=-\pi i d
$$


Equation (I6I) gives, therefore, the effect of the curvatures of the ring, which for ordinary cases will be seen to be small. The resistance and inductance of the ring must, therefore, very approximately follow the same law of variation with the frequency as the straight wire.

The assumption of formula (65) that at high frequencies the magnetic field is symmetrical around the axis of the cross section of the ring is not strictly true. Actually, it will be a little stronger toward the axis of the ring, so that the amplitude of the current is slightly larger in that part of the cross section which is nearest the axis of the ring. This effect, however, will be extremely small and may be neglected.

We have, therefore, with great approximation

$$
\Delta L=-\pi a\left(\mathrm{I}-\frac{4}{x} \frac{Z}{Y}\right)\left[\mathrm{I}-\frac{\mathrm{I}}{2} \frac{\rho^{2}}{a^{2}}\left(\log \frac{8 a}{\rho}-\frac{\mathrm{I}}{3}\right)\right]
$$

or, if terms in $\frac{\rho^{2}}{a^{2}}$ may be neglected,

$$
\begin{aligned}
& \frac{\Delta L}{L}=-\frac{\left(\mathrm{I}-\frac{4}{x} \frac{Z}{Y}\right)}{4 \log \frac{8 a}{\rho}-7} \\
& \left(\frac{\Delta L}{L}\right)_{x=\infty}=-\frac{\mathrm{I}}{4 \log \frac{8 a}{\rho}-7}
\end{aligned}
$$

The values of $\left(\frac{\Delta L}{L}\right)_{x=\infty}$ for various values of the determinative ratio $\frac{8 a}{\rho}$ are tabulated in Table XXIII.

Neglecting the curvature, the change in resistance will be given by the same expression as for the straight wire, that is

$$
\frac{R^{\prime}}{R}=\frac{x}{2} \frac{W}{Y}
$$

The quantities $\frac{x}{2} \frac{W}{Y}$ and $\frac{4}{x} \frac{Z}{Y}$ are to be taken from Table XXII as before, the argument $x$ being given by ( $\left.5_{5} 6\right)$. 
EXAMPLES ILLUSTRATING THE FORMULAS FOR HIGH FREQUENCY

EXAMPLE 82. STRAIGHT WIRE, VERY HIGH FREQUENCY

Let $f=500000$ cycles per second, and $\therefore \lambda=600$ meters

$l=200 \mathrm{~cm}$

$\rho=0.125 \mathrm{~cm}$.

If the wire is of copper, Table XXIV gives $x_{0}=\mathrm{I} 5.146$ for a wire of $\rho=0.1 \mathrm{~cm}$. We find, therefore, $x=15.146 \times 1.25=18.932$. Entering Table XXII with this value of $x$, we find by interpolation, using second differences,

$$
\frac{x}{2} \frac{W}{Y}=\frac{R^{\prime}}{R}=6.95035 \quad \frac{4}{x} \frac{Z}{Y}=0.14923
$$

a slightly more accurate value of the latter may be found by making the interpolation for $\frac{Z}{Y}$.

$$
\frac{2 l}{\rho}=3200 \text { and } \therefore 4 \log \frac{2 l}{\rho}-3=29.284
$$

$$
\text { By (I 54) } \quad\left(\frac{\Delta L}{L}\right)_{x=\infty}=-0.034148
$$

The value found from Table XXIII by interpolation is $0.03416+$. Formula (I47) gives therefore $\frac{\Delta L}{L}=-0.034 \mathrm{I} 48(\mathrm{I}-0.14923)$

By (145)

$$
\begin{aligned}
& =-0.02905^{2} \\
\Delta L & =-85.08 \mathrm{~cm}
\end{aligned}
$$

Recapitulating, the resistance at 50000 cycles per second is 6.95 times as great as with direct current, while the inductance is 85.08 $\mathrm{cm}$ or 2.9052 per cent less than the direct current value. This change of the inductance is 85.08 per cent of the possible change of $100 \mathrm{~cm}$ (0.034I 48 of the total inductance).

If the wire had been of manganin, for which the conductivity was one thirtieth of that of copper, the value of $x$ becomes

$$
x=\mathrm{I} 8.932 \times \sqrt{\frac{I}{30}}=3.4566
$$


and we find

$$
\begin{aligned}
\frac{R^{\prime}}{R}=\mathrm{I} .47620 \quad \frac{4}{x} \frac{Z}{Y} & =.77255 \\
\mathrm{I}-\frac{4}{x} \frac{Z}{Y} & =.22745
\end{aligned}
$$

The resistance is 1.47620 times the value at zero frequency, while the decrease of inductance is only 22.745 per cent of the total possible (0.034148), or 0.007767 .

On the other hand, if the wire had been of iron (conductivity oneseventh of that of copper) and the permeability is assumed as low as 100

$$
\begin{gathered}
x=\sqrt{\mathrm{IOO}} \sqrt{\frac{\mathrm{I}}{7}} \mathrm{I} 8.932=7 \mathrm{I} .556 \\
\frac{R^{\prime}}{R}=25.55 \mathrm{I} \quad \frac{4}{x} \frac{Z}{Y}=0.039526 \quad \mathrm{I}-\frac{4}{x} \frac{Z}{Y}=.96047
\end{gathered}
$$

By $(146)$

$$
\left(\frac{\Delta L}{L}\right)_{x=\infty}=-\frac{100}{128.284}=-0.77950
$$

By (145)

$$
(\Delta L)_{x=\infty}=-10000 \mathrm{~cm}
$$

and the actual changes are

$$
\begin{aligned}
\Delta L & =-9605 \mathrm{~cm} \\
\left(\frac{\Delta L}{L}\right) & =-0.77950 \times .96047=-0.7487
\end{aligned}
$$

The influence of this relatively low permeability is enormous. The resistance is more than twenty-five times its direct current value, while the inductance is less than the direct current value by nearly 75 per cent of the latter, the maximum possible change with this permeability being about 78 per cent.

\section{EXAMPLE 83. STRAIGHT WIRE-LOW FREQUENCY}

If we consider the same wires as in the previous example, except that the frequency is assumed as only $\mathrm{I}, 000$ per second.

Then for copper, $x=0.6774 \times$ I.25 $=0.84675$

$$
\frac{R^{\prime}}{R}=\mathrm{I} .00266 \quad \frac{4}{x} \frac{Z}{Y}=0.99867 \quad \mathrm{I}-\frac{4}{x} \frac{Z}{Y}=0.00 \mathrm{I} 33
$$




$$
\begin{aligned}
& \Delta L=-0.133 \mathrm{~cm} \\
& \frac{\Delta L}{L}=-0.034 \mathrm{I} 48(.00 \mathrm{r} 33)=-0.000045
\end{aligned}
$$

The resistance increase is only 0.266 per cent and the decrease of inductance is only about forty-five millionths of the total.

For manganin, $x=0.84675 \sqrt{\frac{\mathrm{I}}{30}}=0.15^{82}$

$$
\text { By (I5I) } \begin{aligned}
\frac{R^{\prime}}{R}=\mathrm{I} .0000030 \quad \frac{4}{x} \frac{Z}{Y}=\mathrm{I}-0.00000 \mathrm{r} 5 \\
\mathrm{I}-\frac{4}{x} \frac{Z}{Y}=0.00000 \mathrm{r} 5
\end{aligned}
$$

The increase in resistance is about three millionths and the decrease in inductance about five hundred-millionths of the direct current values.

For iron, with $\mu=$ Ioo as before

$$
\begin{aligned}
x & =0.84675 \sqrt{100} \sqrt{\frac{\mathrm{I}}{7}}=3.2004 \\
\frac{R^{\prime}}{R} & =\mathrm{I} .3^{8} 5_{5} \mathrm{I} \quad \quad \frac{4}{x} \frac{Z}{Y}=0.8 \mathrm{I} 39 \mathrm{I} \quad \mathrm{I}-\frac{4}{x} \frac{Z}{Y}=0.18609 \\
\frac{\Delta L}{L} & =-\frac{100}{\mathrm{I} 28.284}(0.18609)=-0.14506
\end{aligned}
$$

That is, the resistance increase is 38.5 per cent, the inductance decrease 14.5 per cent of the direct current values.

\section{EXAMPLE 84. PARALLEL WIRES}

Let us take wires of the same diameter and length as in examples 82 and 83 and consider the same frequencies. The values of $\frac{x}{2} \frac{W}{Y}$ and $\frac{4}{x} \frac{Z}{Y}$ will be the same as those in the cases corresponding in the previous examples. Further, assume that the distance between the centers of the wires is $d=\mathrm{r} .5 \mathrm{~cm}$.

$$
\text { Then } \quad \frac{d}{\rho}=\mathrm{I} 2 \quad 4 \log \frac{d}{\rho}+\mathrm{I}=\mathrm{I} 0.9396
$$


$\therefore$ for nonmagnetic material

$$
\left(\frac{\Delta L}{L}\right)_{x=\infty}=-\frac{\mathrm{I}}{\mathrm{I0.9396}}=-0.09 \mathrm{I} 4 \mathrm{I} 2 .
$$

as may also be found directly with sufficient precision from Table XXIII.

For iron wires, $\mu=\mathrm{IOO}$

$$
\left(\frac{\Delta L}{L}\right)_{x=\infty}=-\frac{\mu}{4 \log \frac{d}{\rho}+\mu}=-\frac{100}{109.94}=-0.9308 .
$$

The results for the cases treated in the previous examples are, therefore, for the parallel wires, as follows:

\begin{tabular}{lrll} 
Material & Frequency & \multicolumn{1}{c}{$\frac{\mathrm{R}^{\prime}}{\mathrm{R}}$} & \multicolumn{1}{c}{$\frac{\Delta \mathrm{L}}{\mathrm{L}}$} \\
Copper & 500000 & 6.9504 & -0.077772 \\
" & 1000 & $\mathrm{I} .00266$ & -0.000122 \\
Manganin & 500000 & $\mathrm{I} .4762$ & -0.020792 \\
" & 1000 & $\mathrm{I} .0000030$ & -0.00000014 \\
Iron ( $\mu=\mathrm{IOO})$ & 500000 & $25.55 \mathrm{I}$ & -0.8940 \\
" & 1000 & $\mathrm{I} .3852$ & -0.1732
\end{tabular}

The following table shows the effect of reducing the radius of

\begin{tabular}{|c|c|c|c|c|c|}
\hline Materi & Frequency & $\mathrm{x}$ & $\left(\frac{\Delta \mathrm{L}}{\mathrm{L}}\right)_{\mathrm{x}=\infty}$ & $\frac{\mathrm{R}^{\prime}}{\mathrm{R}}$ & $\frac{\Delta \mathrm{L}}{\mathrm{L}}$ \\
\hline pp & $\begin{array}{r}500000 \\
1000\end{array}$ & $\begin{array}{l}\text { I. } 5146 \\
0.06774\end{array}$ & $\underset{6}{0.0475^{22}}$ & $\begin{array}{l}\text { I.02682 } \\
\text { I.00000 I I }\end{array}$ & $\begin{array}{l}-0.000636 \\
-2.6 \times 10^{-}\end{array}$ \\
\hline ganin & 500000 & 0.27652 & $475^{22}$ & I.000 & $-7.2 \times 10^{-7}$ \\
\hline " & 1000 & O.0I 237 & & $+1.2 \times 10^{-}$ & $-3 \times 10^{-12}$ \\
\hline $1(\mu=$ & $\begin{array}{r}500000 \\
1000\end{array}$ & $\begin{array}{l}5 \cdot 7245 \\
0.25603\end{array}$ & 0.83303 & $\begin{array}{l}2.2974 \\
\mathrm{I} .000022\end{array}$ & $\begin{aligned} & 0.4273 \\
- & 9.3 \times 10^{-6}\end{aligned}$ \\
\hline
\end{tabular}
cross section to $\rho=0.01 \mathrm{~cm}$

EXAMPLE 85. CIRCULAR RING

Suppose the ring is of copper and that

$$
\rho=0 . \mathrm{I} \mathrm{cm}, \quad a=20 \mathrm{~cm}, \lambda=700 \mathrm{~m}
$$

Then from Table XXIV, $x=\mathrm{I} 4.023$ and from Table XXII,

$$
\frac{R^{\prime}}{R}=5.2173, \mathrm{I}-\frac{4}{x} \frac{Z}{Y}=0.79873
$$




$$
\begin{aligned}
\log \frac{8 a}{\rho} & =\log 1600=7.37776 \\
\frac{\rho}{a} & =0.005
\end{aligned}
$$

By (I62), $(\Delta L)_{x=\infty}=-20 \pi=-62.83 \mathrm{~cm}$.

The correction term in $(\mathrm{I} 6 \mathrm{I})=\mathrm{I}-0.0000880$

By $\left({ }^{6} 6\right)$ or Table XXIII,

and by (164),

$$
\left(\frac{\Delta L}{L}\right)_{x=\infty}=-0.04442
$$

$$
\begin{aligned}
\frac{\Delta L}{L} & =-0.04442 \times 0.79873 \\
& =-0.03548
\end{aligned}
$$

WASHINGTON, January I, I9I I.

NOTE.

After the present paper had gone to press, a third formula for the mutual inductance of coaxial circles was published by Nagaoka (Tokyo Math. Phys., Soc. 6, p. Io; IgII). This formula was given by Nagaoka in the following form:

$$
M=4 \pi \sqrt{A a}\left\{4 \pi q^{*}\left(\frac{\mathrm{I}-4 q^{3}+9 q^{8}-\cdots \cdots}{\mathrm{I}-3 q^{2}+5 q^{6}-\cdots \cdots}\right)\right\}
$$

The general term of the numerator being $(-\mathrm{I})^{n-1} n^{2} q^{n^{2}-1}$ and that of the denominator $(-\mathrm{I})^{m}(2 m+\mathrm{I}) q^{\frac{(2 m+1)^{2}}{4}-1 / 4}$.

The quantity $q$ is calculated from the modulus $k_{1}{ }^{\prime}$, which is complementary to the modulus $k_{1}$ of formula (2). Using the same nomenclature as in section $I$ of this collection we have

$$
\begin{aligned}
q & =\frac{l}{2}+2\left(\frac{l}{2}\right)^{5}+\mathrm{I} 5\left(\frac{l}{2}\right)^{9}+\cdots \\
l & =\frac{\mathrm{I}-\sqrt{k_{1}^{\prime}}}{\mathrm{I}+\sqrt{k_{1}^{\prime}}}=\frac{k_{1}^{2}}{\left(\mathrm{I}+k_{1}^{\prime}\right)\left(\mathrm{I}+\sqrt{k_{1}^{\prime}}\right)^{2}} \\
k_{1} & =\frac{r_{1}-r_{2}}{r_{1}+r_{2}}=\frac{4 A a}{\left(r_{1}+r_{2}\right)^{2}} \quad k_{1}^{\prime}=\frac{2 \sqrt{r_{1} r_{2}}}{r_{1}+r_{2}} \\
r_{1} & =\sqrt{(A+a)^{2}+d^{2}} \quad r_{2}=\sqrt{(A-a)^{2}+d^{2}}
\end{aligned}
$$

The general term of the above formula has been given for the sake of completeness. In general, however, the convergence is so rapid that all but the first terms are negligible. 
As an example of the use of this formula, the calculation for the circles of examples 4 and II above is appended:

$$
\begin{aligned}
& A=a=25 \quad d=4 \\
& R_{1}=\sqrt{25 \mathrm{I} 6}=50 . \mathrm{I} 59744 \quad R_{2}=4 \\
& k_{1}{ }^{\prime}=\frac{4 \sqrt{25^{\mathrm{I} 6}}}{4+\sqrt{2 \mathrm{II}^{\mathrm{I}}}} \quad \sqrt{k_{1}^{\prime}}=0.72323683 \\
& \frac{l}{2}=0.080303278 \quad q=0.080309959 \\
& \mathrm{I}-4 q^{3}+9 q^{8}=0.997928 \mathrm{I} 2 \quad \mathrm{I}-3 q^{2}+5 q^{6}=0.98065227 \\
& \log q^{*}=\overline{\mathrm{I}} \text {.I785770 } \quad \therefore M=606.0676 \mathrm{~cm}
\end{aligned}
$$

which agrees very closely with the value 606.0674 found in examples 4 and II.

On expansion the above formula becomes

$$
\mathrm{M}=4 \pi \sqrt{\mathrm{Aa}}\left\{4 \pi \mathrm{q}^{\frac{3}{3}}\left(\mathrm{I}+3 \mathrm{q}^{2}-4 \mathrm{q}^{3}+9 \mathrm{q}^{4}-\mathrm{I} 2 \mathrm{q}^{5}+\cdots\right)\right\}
$$

which suggests that the quantity $q$ in this expression is equal to the square of the corresponding quantity in formula (8) above. The truth of this proposition may be established by expressing Landen's transformation in terms of $q$ functions.

As regards numerical calculation, therefore, this last formula of Nagaoka is entirely equivalent to his earlier formula (8). 


\section{APPENDIX}

TABLES OF CONSTANTS AND FUNCTIONS USEFUL IN THE CALCULATION OF MUTUAL AND SELF-INDUCTANCE 
TABLE I

Maxwell's Table of Values of $\log \frac{M}{4 \pi \sqrt{A a}}=\left[\left(\frac{2}{k}-k\right) F-\frac{2}{k} E\right]$

(For use with Formula (1))

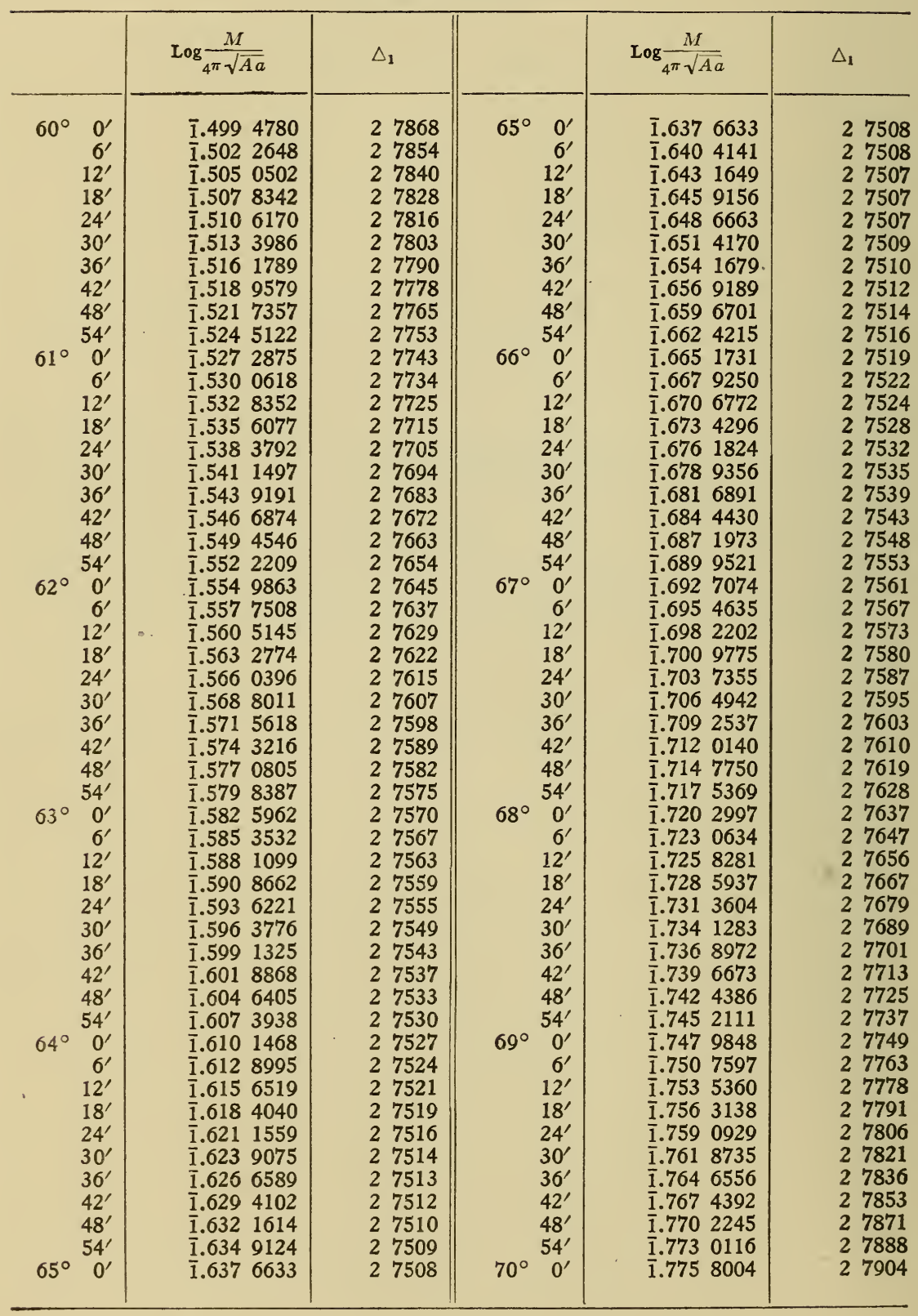


TABLE I-Continued

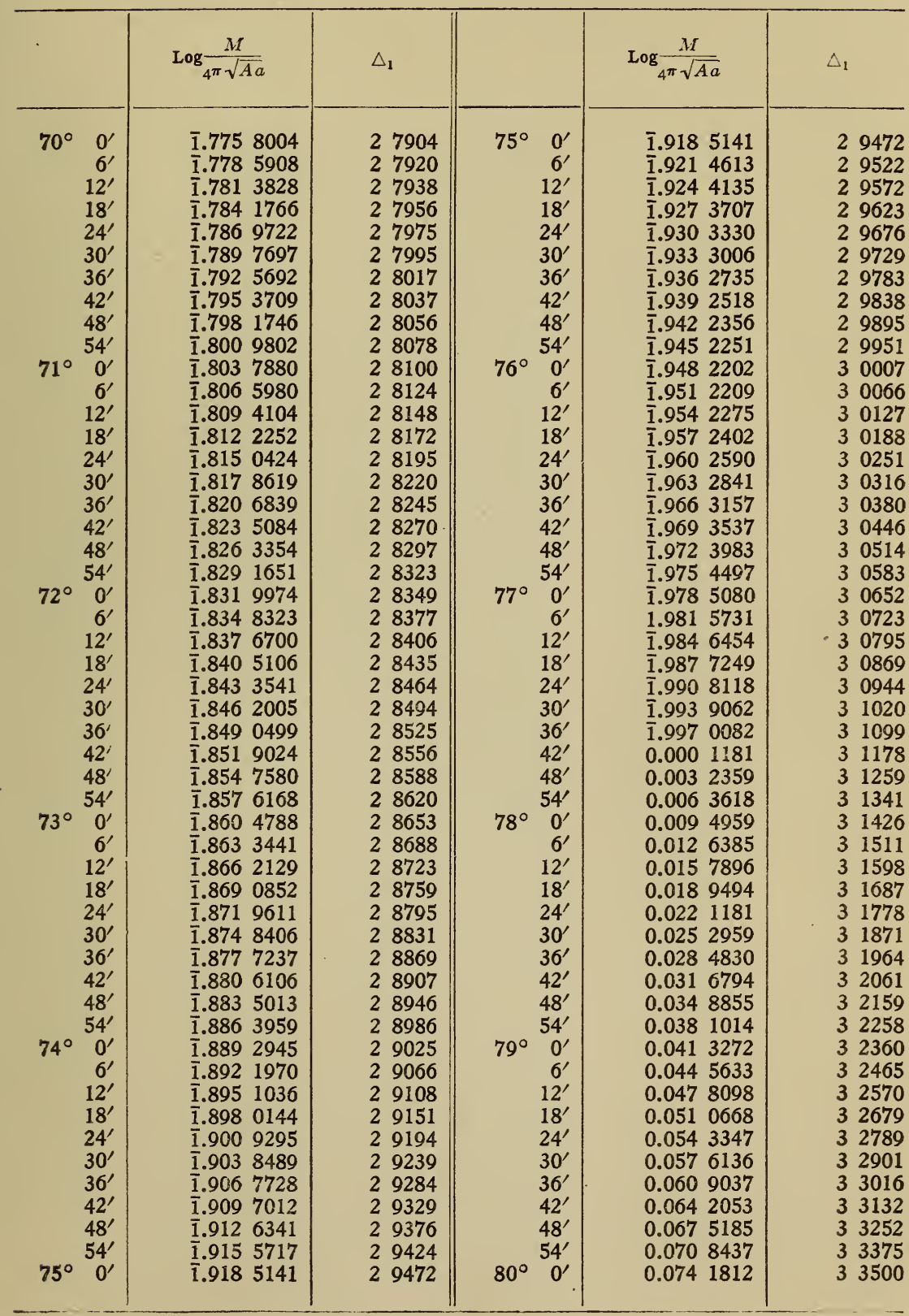


TABLE I-Continued

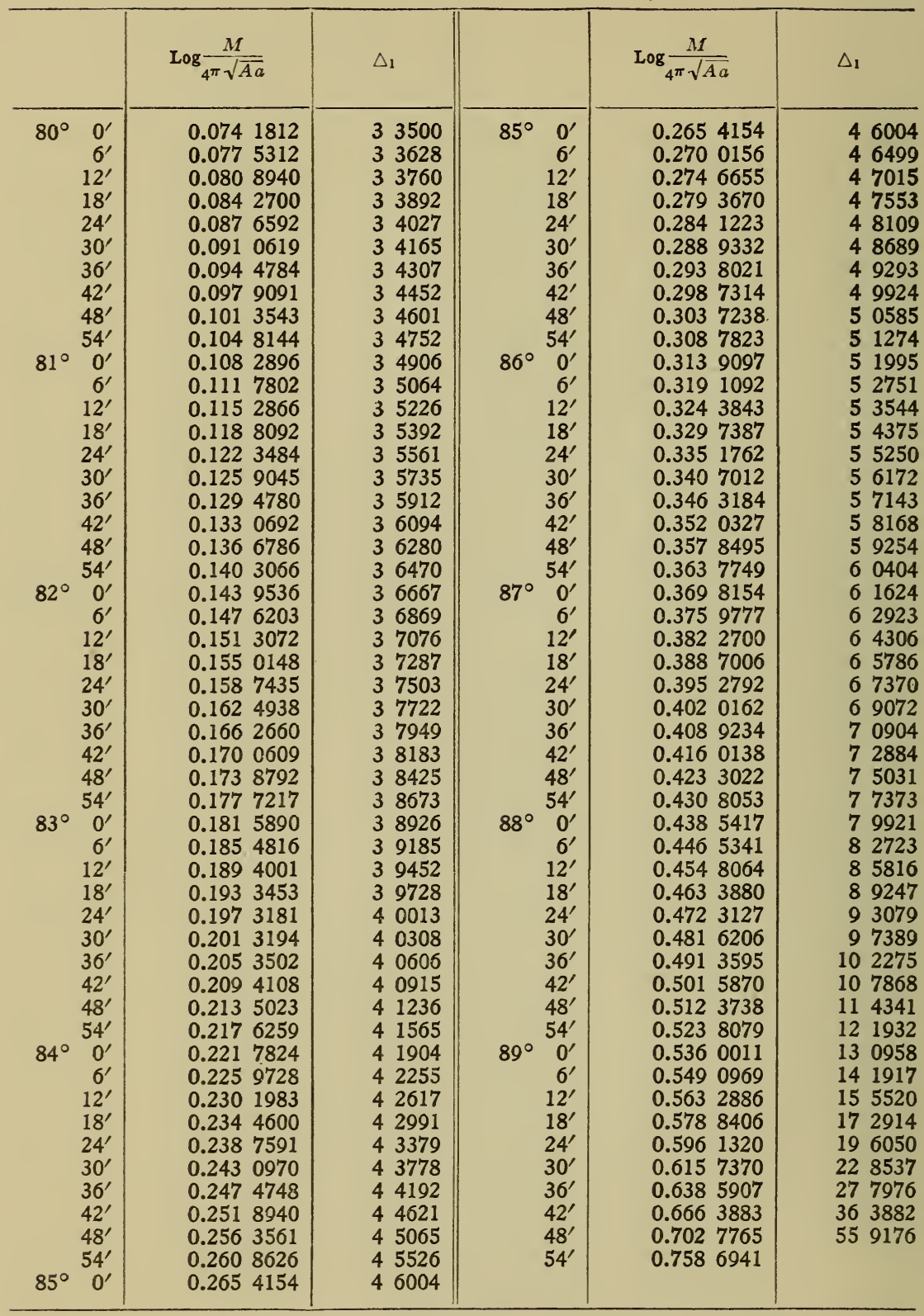

The above table has been recalculated and some of the values corrected in the last place. The values given are sufficiently accurate to give $M$ within one part in a million. 
TABLE II

Giving the Values of $\log F$ and $\log E$ as Functions of $\tan \gamma$. (See p. 20)

\begin{tabular}{r|c|c|c|c|c}
\hline $\tan \gamma$ & Log F & \multicolumn{1}{|c|}{ F } & Log $E$ & E \\
\cline { 2 - 3 } 0.1 & 0.1971996 & 1.5747065 & 0.1950415 & 1.5669007 \\
0.2 & 0.2003678 & 1.5862361 & 0.1918928 & 1.5555817 \\
0.3 & 0.2054261 & 1.6048192 & 0.1869144 & 1.5378514 \\
0.4 & 0.2120849 & 1.6296146 & 0.1804536 & 1.5151429 \\
0.5 & 0.2200096 & 1.6596236 & 0.1729048 & 1.4890346 \\
0.6 & 0.2288634 & 1.6938051 & 0.1646557 & 1.4610185 \\
0.7 & 0.2383385 & 1.7311652 & 0.1560492 & 1.4323502 \\
0.8 & 0.2481728 & 1.7708135 & 0.1473640 & 1.4039900 \\
0.9 & 0.2581561 & 1.8119912 & 0.1388116 & 1.3766121 \\
1.0 & 0.2681272 & 1.8540745 & 0.1305409 & 1.3506441 \\
1.5 & 0.3147473 & 2.0641787 & 0.0955992 & 1.2462329 \\
2.0 & 0.3535711 & 2.2572057 & 0.0713258 & 1.1784897 \\
2.5 & 0.3852192 & 2.4278352 & 0.0547850 & 1.1344491 \\
3.0 & 0.4112984 & 2.5780917 & 0.0432738 & 1.1047748 \\
4.0 & 0.4518237 & 2.8302429 & 0.0289324 & 1.0688885 \\
5.0 & 0.4821752 & 3.0351154 & 0.0207426 & 1.0489205 \\
7.5 & 0.5341061 & 3.4206300 & 0.0109567 & 1.0255497 \\
10.0 & 0.5682672 & 3.7005581 & 0.0068338 & 1.0158598 \\
12.5 & 0.5932708 & 3.9198622 & 0.0047004 & 1.0108819 \\
& & & & & \\
\hline & & & & & \\
\hline
\end{tabular}

TABLE III

Values of the Constant $K$ as Functions of $x / A$ and $a / A$

(For use in Formula (57))

\begin{tabular}{c|c|c|c|c|c|c|c}
\hline $\mathrm{x} / \mathrm{A}$ & $=.50$ & .75 & 1 & 1.25 & 1.50 & 1.75 & 2 \\
\hline \hline $\mathrm{a} / \mathrm{A}$ & & & & & & & \\
\hline 0.50 & 9.39283 & 12.30385 & 14.27982 & 15.62795 & 16.56549 & 17.23299 & 17.71973 \\
0.55 & 9.52044 & 12.40135 & 14.34594 & 15.67140 & 16.59411 & 17.25215 & 17.73283 \\
0.60 & 9.66358 & 12.50816 & 14.41766 & 15.71837 & 16.62503 & 17.27286 & 17.74701 \\
0.65 & 9.82296 & 12.62412 & 14.49474 & 15.76867 & 16.65813 & 17.29504 & 17.76221 \\
0.70 & 9.99921 & 12.74897 & 14.57688 & 15.82212 & 16.69330 & 17.31865 & 17.77841 \\
0.75 & 10.19272 & 12.88232 & 14.66377 & 15.87850 & 16.73039 & 17.34357 & 17.79554 \\
\hline
\end{tabular}




\section{TABLE IV}

Values of the Constant $Q$ in Formula (74), $L_{s}=n^{2} a Q$

For the self-inductance of a single-layer winding on a solenoid; $n$ is the whole number of turns of wire in the winding and $a$ is the mean radius. The corrections by Tables VII and VIII must be made to get $L$ from $L_{s}$ as usual. (See p. I 22.)

In the following table $2 a$ is the diameter, $b$ is the length, and therefore $2 a \mid b=\tan \gamma$. (See Fig. 33.)

\begin{tabular}{l|c||c|c}
\hline $2 \frac{a}{b}=\tan \gamma$ & $Q$ & $2_{\tilde{b}}^{a}=\tan \gamma$ & $Q$ \\
\hline 0.20 & 3.63240 & 1.80 & 19.57938 \\
0.30 & 5.23368 & 2.00 & 20.74631 \\
0.40 & 6.71017 & 2.20 & 21.82049 \\
0.50 & 8.07470 & 2.40 & 22.81496 \\
0.60 & 9.33892 & 2.60 & 23.74013 \\
0.70 & 10.51349 & 2.80 & 24.60482 \\
0.80 & 11.60790 & 3.00 & 25.41613 \\
0.90 & 12.63059 & 3.20 & 26.18009 \\
1.00 & 13.58892 & 3.40 & 26.90177 \\
1.20 & 15.33799 & 3.60 & 27.58548 \\
1.40 & 16.89840 & 3.80 & 28.23494 \\
1.60 & 18.30354 & 4.00 & 28.85335 \\
\hline
\end{tabular}

For an explanation of the above formula, see page i 8 . 


\section{TABLE V}

Constants $A$ and $B$ for Strasser's Formula (82)

$$
\begin{aligned}
A & =2 \log _{e}[(n-\mathrm{r}) !(n-2) ! \cdots \cdots \mathrm{I}] \\
B & =3\left[(n-2) 2^{2} \log _{e} 2+(n-3) 3^{2} \log _{e} 3+\cdots(n-\mathrm{r})^{2} \log _{e}(n-\mathrm{I})\right]
\end{aligned}
$$

\begin{tabular}{r|l|c||c|c|c}
\hline n & A & B & n & A & B \\
\cline { 2 - 3 } 1 & 0 & 0 & 16 & 354.396 & 35693 \\
2 & 0 & 0 & 17 & 415.739 & 46775 \\
3 & 1.38629 & 8.318 & 18 & 482.75 & 60314 \\
4 & 4.96981 & 46.298 & 19 & 555.54 & 76662 \\
5 & 11.3259 & 150.82 & 20 & 634.22 & 96198 \\
6 & 20.9009 & 376.05 & 21 & 718.89 & 119330 \\
7 & 34.0594 & 794.79 & 22 & 809.65 & 146490 \\
8 & 51.1097 & 1499.58 & 23 & 906.59 & 178140 \\
9 & 72.3189 & 2603.62 & 24 & 1009.81 & 214760 \\
10 & 97.9226 & 4241.59 & 25 & 1119.38 & 256880 \\
11 & 128.131 & 6570.33 & 26 & 1235.38 & 305030 \\
12 & 163.136 & 9769.51 & 27 & 1357.91 & 359790 \\
13 & 203.110 & 14042.2 & 28 & 1487.02 & 421750 \\
14 & 248.215 & 19615.3 & 29 & 1622.80 & 491560 \\
15 & 298.597 & 26740.1 & 30 & 1765.32 & 569860 \\
\hline
\end{tabular}

We have recently recomputed Strasser's constants, finding several errors which are corrected here. 
TABLE VI

Table of Constants for Stefan's Formula (90)

\begin{tabular}{l|c|c||c|c|c}
\hline$b / c$ or $c / b$ & $y_{1}$ & $y_{2}$ & $b / c$ or $c / b$ & $y_{1}$ & \multicolumn{1}{|c}{$y_{2}$} \\
\cline { 1 - 2 } 0.00 & 0.50000 & 0.1250 & 0.55 & 0.80815 & 0.3437 \\
0.05 & .54899 & .1269 & 0.60 & .81823 & .3839 \\
0.10 & .59243 & .1325 & 0.65 & .82648 & .4274 \\
0.15 & .63102 & .1418 & 0.70 & .83311 & .4739 \\
0.20 & .66520 & .1548 & 0.75 & .83831 & .5234 \\
0.25 & .69532 & .1714 & 0.80 & .84225 & .5760 \\
0.30 & .72172 & .1916 & 0.85 & .84509 & .6317 \\
0.35 & .74469 & .2152 & 0.90 & .84697 & .6902 \\
0.40 & .76454 & .2423 & 0.95 & .84801 & .7518 \\
0.45 & .78154 & .2728 & 1.00 & .84834 & .8162 \\
0.50 & .79600 & .3066 & & & \\
\hline
\end{tabular}

There is in general no difficulty in obtaining $y_{1}$ and $y_{2}$ with suffcient accuracy by interpolation from this table, using second and in some cases third differences. The only case which may give trouble is when $b / c$ or $c / b$ is less than O.I. In such cases, however, Stefan's formula does not give precise results, and the errors in the interpolation will not be important. 
${ }_{\text {Grover ] Formulas for Mutual and Self-Inductance }}^{\text {Rosa }}$

\section{TABLE VII}

Values of Correction Term A, Depending on the Ratio $\frac{d}{D}$ of the Diameters of Bare and Covered Wire on the Single Layer Coil

(For use in Formula (80))

$$
\mathrm{A}=\log _{e}\left(1.7452 \frac{d}{D}\right)
$$

\begin{tabular}{|c|c|c|c|c|c|c|c|c|c|}
\hline$\frac{d}{D}$ & A & $\Delta_{1}$ & $\frac{d}{D}$ & A & $\triangle_{1}$ & $\frac{d}{D}$ & A & $\triangle_{1}$ & $\Delta_{8}$ \\
\hline 1.00 & 0.5568 & -100 & 0.75 & 0.2691 & -134 & 0.50 & -0.1363 & -202 & -4 \\
\hline .99 & .5468 & -101 & .74 & .2557 & -136 & .49 & -.1565 & -206 & -5 \\
\hline .98 & .5367 & -103 & .73 & .2421 & -138 & .48 & -.1771 & -211 & -4 \\
\hline .97 & .5264 & -104 & .72 & .2283 & -140 & .47 & -.1982 & -215 & -4 \\
\hline .96 & .5160 & -105 & .71 & .2143 & -142 & .46 & -.2197 & -219 & -6 \\
\hline 0.95 & 0.5055 & -106 & 0.70 & 0.2001 & -144 & 0.45 & -0.2416 & -225 & -5 \\
\hline .94 & .4949 & -107 & .69 & .1857 & -146 & .44 & -.2641 & -230 & -5 \\
\hline .93 & .4842 & -108 & .68 & .1711 & -148 & .43 & -.2871 & -235 & -6 \\
\hline .92 & .4734 & -109 & .67 & .1563 & -150 & .42 & -.3106 & -241 & -6 \\
\hline .91 & .4625 & -110 & .66 & .1413 & -152 & .41 & -.3347 & -247 & -6 \\
\hline 0.90 & 0.4515 & -112 & 0.65 & 0.1261 & -155 & 0.40 & -0.3594 & -253 & -7 \\
\hline .89 & .4403 & -113 & .64 & .1106 & -157 & .39 & -.3847 & -260 & -7 \\
\hline .88 & .4290 & -114 & .63 & .0949 & -160 & .38 & -.41 & -267 & -7 \\
\hline .87 & .4176 & -116 & .62 & .0789 & -163 & .37 & -.4374 & -274 & -7 \\
\hline .86 & .4060 & -117 & .61 & .0626 & -166 & .36 & -.4648 & -281 & -9 \\
\hline 0.85 & 0.3943 & -118 & 0.60 & 0.0460 & -168 & 0.35 & -0.4929 & -290 & -9 \\
\hline .84 & .3825 & -120 & .59 & .0292 & -171 & .34 & -.5219 & -299 & -9 \\
\hline .83 & .3705 & -121 & .58 & +.0121 & -174 & .33 & -.5518 & -308 & -9 \\
\hline .82 & .3584 & -123 & .57 & -.0053 & -177 & .32 & -.5826 & -317 & -9 \\
\hline .81 & .3461 & -124 & .56 & -.0230 & -180 & .31 & -.6143 & -328 & -11 \\
\hline 0.80 & 0.3337 & -126 & 0.55 & -0.0410 & -184 & 0.30 & -0.6471 & -339 & -12 \\
\hline .79 & .3211 & -127 & .54 & -.0594 & -187 & .29 & -.6810 & -351 & -13 \\
\hline .78 & .3084 & -129 & .53 & -.0781 & -190 & .28 & -.7161 & -364 & -13 \\
\hline .77 & .2955 & -131 & .52 & -.0971 & -194 & .27 & -.7525 & -377 & -15 \\
\hline .76 & .2824 & -133 & .51 & -.1165 & -198 & .26 & -.7902 & -392 & -16 \\
\hline 0.75 & 0.2691 & & 0.50 & -0.1363 & & 0.25 & -0.8294 & & \\
\hline
\end{tabular}


TABLE VII-Continued

\begin{tabular}{|c|c|c|c|c|c|c|c|}
\hline$\frac{\mathrm{d}}{\mathrm{D}}$ & A. & $\triangle_{1}$ & $\triangle_{2}$ & $\frac{\mathrm{d}}{\mathrm{D}}$ & $\mathbf{A}$ & $\Delta_{1}$ & $\Delta_{2}$ \\
\hline 0.25 & -0.8294 & -408 & -18 & 0.10 & -1.7457 & -1054 & -124 \\
\hline .24 & -.8702 & -426 & -19 & .09 & -1.8511 & -1178 & -157 \\
\hline .23 & -.9128 & -445 & -20 & .08 & -1.9689 & -1335 & -206 \\
\hline .22 & -.9573 & -465 & -23 & .07 & -2.1024 & -1541 & -283 \\
\hline .21 & -1.0038 & -488 & -25 & .06 & -2.2565 & -1824 & -407 \\
\hline 0.20 & -1.0526 & -513 & -28 & 0.05 & -2.4389 & -2231 & -646 \\
\hline .19 & -1.1039 & -541 & -30 & .04 & -2.6620 & -2877 & -1177 \\
\hline .18 & -1.1580 & -571 & -35 & .03 & -2.9497 & -4054 & -2878 \\
\hline .17 & -1.2151 & -606 & -39 & .02 & -3.3551 & -6932 & \\
\hline .16 & -1.2757 & -645 & -45 & .01 & -4.0483 & & \\
\hline 0.15 & -1.3402 & -690 & -51 & & & & \\
\hline .14 & -1.4092 & -741 & -60 & & & & \\
\hline .13 & -1.4833 & -801 & -70 & & & & \\
\hline .12 & -1.5634 & -870 & -83 & & & & \\
\hline .11 & -1.6504 & -953 & -101 & & & & \\
\hline 0.10 & -1.7457 & & & & & & \\
\hline
\end{tabular}




\section{TABLE VIII}

Values of the Correction Term B, Depending on the Number of Turns of Wire on the Single Layer Coil

(For use in Formula (80))

$$
B=\frac{2}{n} \stackrel{n}{\Sigma}_{\mathrm{I}}^{n-\mathrm{r}} m \log _{e} \frac{m}{R_{m}}
$$

where $R_{m}$ is geometric mean distance of the sections of the current sheet whose centers coincide with those of the wires. (See this Bulletin, 2, p. I68, equat. (I I); rgo6.)

\begin{tabular}{c|c||c|c}
\hline Number of Turns $=\mathbf{n}$ & B & Number of Turns=n & B \\
\hline 1 & 0.0000 & 50 & 0.3186 \\
2 & .1137 & 60 & .3216 \\
3 & .1663 & 70 & .3239 \\
4 & .1973 & 80 & .3257 \\
5 & .2180 & 90 & .3270 \\
6 & .2329 & 100 & .3280 \\
7 & .2443 & 125 & .3298 \\
8 & .2532 & 150 & .3311 \\
9 & .2604 & 175 & .3321 \\
10 & .2664 & 200 & .3328 \\
15 & .2857 & 300 & .3343 \\
20 & .2974 & 400 & .3351 \\
25 & .3042 & 500 & .3356 \\
30 & .3083 & 600 & .3359 \\
35 & .3119 & 700 & .3361 \\
40 & .3148 & 800 & .3363 \\
45 & .3169 & 900 & .3364 \\
50 & .3186 & 1000 & .3365 \\
& & & \\
\hline
\end{tabular}




\section{TABLE IX}

Value of the Constant $A_{s}$ as a Function of $t / a$, $t$ being the Depth of the Winding and a the Mean Radius

$$
A_{s}=0.6949-\frac{t^{2}}{96 a^{2}}\left(\log _{e} \frac{8 a}{t}+2.76\right)
$$

(For use in Formula (91))

\begin{tabular}{l|l}
\hline$t / a$ & $A_{s}$ \\
\hline 0 & 0.6949 \\
0.10 & 0.6942 \\
0.15 & 0.6933 \\
0.20 & 0.6922 \\
0.25 & 0.6909 \\
\hline
\end{tabular}

\section{TABLE X}

Values of the Correction Term $B_{s}$ depending on the Number of Turns of Square Conductor on Single Layer Coil

(For use in Formula (91))

$$
B_{s}=\frac{2}{n} \sum_{\mathrm{I}}^{n-\mathrm{I}}\left(m \log \frac{R_{m}^{\prime}}{R_{m}}\right)
$$

where $R_{m}^{\prime}=$ geom. mean distance for the two squares

$R_{m}=$ " " " " " elements of the current sheet. (See this Bulletin, 4, p. 373; 1907.)

\begin{tabular}{c|c||c|c||c|c}
\hline $\begin{array}{c}\text { Number of } \\
\text { Turns }\end{array}$ & $B_{s}$ & $\begin{array}{c}\text { Number of } \\
\text { Turns }\end{array}$ & $B_{s}$ & $\begin{array}{c}\text { Number of } \\
\text { Turns }\end{array}$ & $B_{s}$ \\
\hline 1 & 0.0000 & 11 & 0.2844 & 21 & 0.3116 \\
2 & .1202 & 12 & .2888 & 22 & .3131 \\
3 & .1753 & 13 & .2927 & 23 & .3145 \\
4 & .2076 & 14 & .2961 & 24 & .3157 \\
5 & .2292 & 15 & .2991 & 25 & .3169 \\
6 & .2446 & 16 & .3017 & 26 & .3180 \\
7 & .2563 & 17 & .3041 & 27 & .3190 \\
8 & .2656 & 18 & .3062 & 28 & .3200 \\
9 & .2730 & 19 & .3082 & 29 & .3209 \\
10 & .2792 & 20 & .3099 & 30 & .3218 \\
\hline
\end{tabular}


TABLE XI

Table of Napierian Logarithms to Nine Decimal Places for Numbers from 1 to 100

\begin{tabular}{|c|c|c|c|}
\hline $\begin{array}{l}1 \\
2 \\
3 \\
4 \\
5\end{array}$ & $\begin{array}{lll}0.000 & 000 & 000 \\
0.693 & 147 & 181 \\
1.098 & 612 & 289 \\
1.386 & 294 & 361 \\
1.609 & 437 & 912\end{array}$ & $\begin{array}{l}51 \\
52 \\
53 \\
54 \\
55\end{array}$ & $\begin{array}{lll}3.931 & 825 & 633 \\
3.951 & 243 & 719 \\
3.970 & 291 & 914 \\
3.988 & 984 & 047 \\
4.007 & 333 & 185\end{array}$ \\
\hline $\begin{array}{r}6 \\
7 \\
8 \\
9 \\
10\end{array}$ & $\begin{array}{lll}1.791 & 759 & 469 \\
1.945 & 910 & 149 \\
2.079 & 441 & 542 \\
2.197 & 224 & 577 \\
2.302 & 585 & 093\end{array}$ & $\begin{array}{l}56 \\
57 \\
58 \\
59 \\
60\end{array}$ & $\begin{array}{lll}4.025 & 351 & 691 \\
4.043 & 051 & 268 \\
4.060 & 443 & 011 \\
4.077 & 537 & 444 \\
4.094 & 344 & 562\end{array}$ \\
\hline $\begin{array}{l}11 \\
12 \\
13 \\
14 \\
15\end{array}$ & $\begin{array}{lll}2.397 & 895 & 273 \\
2.484 & 906 & 650 \\
2.564 & 949 & 357 \\
2.639 & 057 & 330 \\
2.708 & 050 & 201\end{array}$ & $\begin{array}{l}61 \\
62 \\
63 \\
64 \\
65\end{array}$ & $\begin{array}{lll}4.110 & 873 & 864 \\
4.127 & 134 & 385 \\
4.143 & 134 & 726 \\
4.158 & 883 & 083 \\
4.174 & 387 & 270\end{array}$ \\
\hline $\begin{array}{l}16 \\
17 \\
18 \\
19 \\
20\end{array}$ & $\begin{array}{lll}2.772 & 588 & 722 \\
2.833 & 213 & 344 \\
2.890 & 371 & 758 \\
2.944 & 438 & 979 \\
2.995 & 732 & 274\end{array}$ & $\begin{array}{l}66 \\
67 \\
68 \\
69 \\
70\end{array}$ & $\begin{array}{lll}4.189 & 654 & 742 \\
4.204 & 692 & 619 \\
4.219 & 507 & 705 \\
4.234 & 106 & 505 \\
4.248 & 495 & 242\end{array}$ \\
\hline $\begin{array}{l}21 \\
22 \\
23 \\
24 \\
25\end{array}$ & $\begin{array}{lll}3.044 & 522 & 438 \\
3.091 & 042 & 453 \\
3.135 & 494 & 216 \\
3.178 & 053 & 830 \\
3.218 & 875 & 825\end{array}$ & $\begin{array}{l}71 \\
72 \\
73 \\
74 \\
75\end{array}$ & $\begin{array}{lll}4.262 & 679 & 877 \\
4.276 & 666 & 119 \\
4.290 & 459 & 441 \\
4.304 & 065 & 093 \\
4.317 & 488 & 114\end{array}$ \\
\hline $\begin{array}{l}26 \\
27 \\
28 \\
29 \\
30\end{array}$ & $\begin{array}{lll}3.258 & 096 & 538 \\
3.295 & 836 & 866 \\
3.332 & 204 & 510 \\
3.367 & 295 & 830 \\
3.401 & 197 & 382\end{array}$ & $\begin{array}{l}76 \\
77 \\
78 \\
79 \\
80\end{array}$ & $\begin{array}{lll}4.330 & 733 & 340 \\
4.343 & 805 & 422 \\
4.356 & 708 & 827 \\
4.369 & 447 & 852 \\
4.382 & 026 & 635\end{array}$ \\
\hline $\begin{array}{l}31 \\
32 \\
33 \\
34 \\
35\end{array}$ & $\begin{array}{lll}3.433 & 987 & 204 \\
3.465 & 735 & 903 \\
3.496 & 507 & 561 \\
3.526 & 360 & 525 \\
3.555 & 348 & 061\end{array}$ & $\begin{array}{l}81 \\
82 \\
83 \\
84 \\
85\end{array}$ & $\begin{array}{lll}4.394 & 339 & 155 \\
4.406 & 719 & 247 \\
4.418 & 840 & 608 \\
4.430 & 816 & 799 \\
4.442 & 651 & 256\end{array}$ \\
\hline $\begin{array}{l}36 \\
37 \\
38 \\
39 \\
40\end{array}$ & $\begin{array}{lll}3.583 & 518 & 938 \\
3.610 & 917 & 913 \\
3.637 & 586 & 160 \\
3.663 & 561 & 646 \\
3.688 & 879 & 454\end{array}$ & $\begin{array}{l}86 \\
87 \\
88 \\
89 \\
90\end{array}$ & $\begin{array}{lll}4.454 & 347 & 296 \\
4.465 & 908 & 119 \\
4.477 & 336 & 814 \\
4.488 & 636 & 370 \\
4.499 & 809 & 670\end{array}$ \\
\hline $\begin{array}{l}41 \\
42 \\
43 \\
44 \\
45\end{array}$ & $\begin{array}{lll}3.713 & 572 & 067 \\
3.737 & 669 & 618 \\
3.761 & 200 & 116 \\
3.784 & 189 & 634 \\
3.806 & 662 & 490\end{array}$ & $\begin{array}{l}91 \\
92 \\
93 \\
94 \\
95\end{array}$ & $\begin{array}{lll}4.510 & 859 & 507 \\
4.521 & 788 & 577 \\
4.532 & 599 & 493 \\
4.543 & 294 & 782 \\
4.553 & 876 & 892\end{array}$ \\
\hline $\begin{array}{l}46 \\
47 \\
48 \\
49 \\
50\end{array}$ & $\begin{array}{lll}3.828 & 641 & 396 \\
3.850 & 147 & 602 \\
3.871 & 201 & 011 \\
3.891 & 820 & 298 \\
3.912 & 023 & 005\end{array}$ & $\begin{array}{r}96 \\
97 \\
98 \\
99 \\
100\end{array}$ & $\begin{array}{lll}4.564 & 348 & 191 \\
4.574 & 710 & 979 \\
4.584 & 967 & 479 \\
4.595 & 119 & 850 \\
4.605 & 170 & 186\end{array}$ \\
\hline
\end{tabular}

$\log { }_{1525}=\log 25+\log 6 \mathrm{I} ; \log 9.8=\log 98-\log$ I0, etc. 


\section{TABLE XII}

Values of $F$ and $E$

The following table of elliptic integrals of the first and second kind is taken from Legendre's Traité des Fonctions Elliptiques, Volume 2, Table VIII :

\begin{tabular}{|c|c|c|c|c|c|c|c|}
\hline & F & $\Delta_{1}$ & $\Delta_{2}$ & & $E$ & $\Delta_{1}$ & $\Delta_{2}$ \\
\hline $\begin{array}{l}0^{\circ} \\
1 \\
2 \\
3 \\
4 \\
5\end{array}$ & 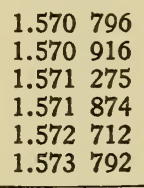 & $\begin{array}{ll} & 120 \\
& 359 \\
& 599 \\
& 839 \\
1 & 080 \\
1 \quad 321 \\
\end{array}$ & $\begin{array}{l}239 \\
240 \\
240 \\
241 \\
241 \\
243 \\
\end{array}$ & $\begin{array}{l}0^{\circ} \\
1 \\
2 \\
3 \\
4 \\
5 \\
\end{array}$ & $\begin{array}{ll}1.570 & 796 \\
1.570 & 677 \\
1.570 & 318 \\
1.569 & 720 \\
1.568 & 884 \\
1.567 & 809 \\
\end{array}$ & $\begin{array}{l}-\quad 120 \\
-\quad 359 \\
-\quad 598 \\
-\quad 836 \\
-1075 \\
-1312 \\
\end{array}$ & $\begin{array}{l}-239 \\
-239 \\
-239 \\
-238 \\
-238 \\
-237 \\
\end{array}$ \\
\hline $\begin{array}{r}6 \\
7 \\
8 \\
9 \\
10\end{array}$ & $\begin{array}{ll}1.575 & 114 \\
1.576 & 678 \\
1.578 & 486 \\
1.580 & 541 \\
1.582 & 843\end{array}$ & $\begin{array}{ll}1 & 564 \\
1 & 808 \\
2 & 054 \\
2 & 302 \\
2 & 551\end{array}$ & $\begin{array}{l}244 \\
246 \\
247 \\
249 \\
252\end{array}$ & $\begin{array}{r}6 \\
7 \\
8 \\
9 \\
10\end{array}$ & $\begin{array}{ll}1.566 & 497 \\
1.564 & 948 \\
1.563 & 162 \\
1.561 & 142 \\
1.558 & 887\end{array}$ & $\begin{array}{ll}-1 & 549 \\
-1 & 785 \\
-2 & 020 \\
-2 & 255 \\
-2 & 487\end{array}$ & $\begin{array}{l}-236 \\
-235 \\
-234 \\
-233 \\
-232\end{array}$ \\
\hline $\begin{array}{l}11 \\
12 \\
13 \\
14 \\
15\end{array}$ & $\begin{array}{ll}1.585 & 394 \\
1.588 & 197 \\
1.591 & 254 \\
1.594 & 568 \\
1.598 & 142\end{array}$ & $\begin{array}{ll}2 & 803 \\
3 & 057 \\
3 & 314 \\
3 & 574 \\
3 & 836\end{array}$ & $\begin{array}{l}254 \\
257 \\
260 \\
263 \\
266\end{array}$ & $\begin{array}{l}11 \\
12 \\
13 \\
14 \\
15\end{array}$ & $\begin{array}{ll}1.556 & 400 \\
1.553 & 681 \\
1.550 & 732 \\
1.547 & 554 \\
1.544 & 150\end{array}$ & $\begin{array}{ll}-2 & 719 \\
-2 & 949 \\
-3 & 177 \\
-3 & 404 \\
-3 & 629\end{array}$ & $\begin{array}{l}-230 \\
-228 \\
-227 \\
-225 \\
-223\end{array}$ \\
\hline $\begin{array}{l}16 \\
17 \\
18 \\
19 \\
20\end{array}$ & $\begin{array}{ll}1.601 & 978 \\
1.606 & 081 \\
1.610 & 454 \\
1.615 & 101 \\
1.620 & 026\end{array}$ & $\begin{array}{ll}4 & 103 \\
4 & 373 \\
4 & 647 \\
4 & 925 \\
5 & 208\end{array}$ & $\begin{array}{l}270 \\
274 \\
278 \\
283 \\
288\end{array}$ & $\begin{array}{l}16 \\
17 \\
18 \\
19 \\
20\end{array}$ & $\begin{array}{ll}1.540 & 521 \\
1.536 & 670 \\
1.532 & 597 \\
1.528 & 306 \\
1.523 & 799\end{array}$ & $\begin{array}{ll}-3 & 852 \\
-4 & 073 \\
-4 & 291 \\
-4 & 507 \\
-4 & 721\end{array}$ & $\begin{array}{l}-221 \\
-218 \\
-216 \\
-214 \\
-211\end{array}$ \\
\hline $\begin{array}{l}21 \\
22 \\
23 \\
24 \\
25\end{array}$ & $\begin{array}{l}1.625234 \\
1.630729 \\
1.636517 \\
1.642604 \\
1.648995 \\
\end{array}$ & $\begin{array}{ll}5 & 495 \\
5 & 788 \\
6 & 087 \\
6 & 391 \\
6 & 702 \\
\end{array}$ & $\begin{array}{l}293 \\
298 \\
304 \\
311 \\
317\end{array}$ & $\begin{array}{l}21 \\
22 \\
23 \\
24 \\
25 \\
\end{array}$ & 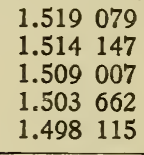 & $\begin{array}{ll}-4 & 932 \\
-5 & 140 \\
-5 & 345 \\
-5 & 547 \\
-5 & 746\end{array}$ & $\begin{array}{l}-208 \\
-205 \\
-202 \\
-199 \\
-196\end{array}$ \\
\hline $\begin{array}{l}26 \\
27 \\
28 \\
29 \\
30\end{array}$ & 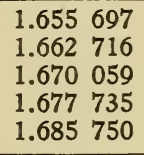 & $\begin{array}{ll}7 & 019 \\
7 & 343 \\
7 & 675 \\
8 & 015 \\
8 & 364\end{array}$ & $\begin{array}{l}324 \\
332 \\
340 \\
349 \\
358\end{array}$ & $\begin{array}{l}26 \\
27 \\
28 \\
29 \\
30\end{array}$ & $\begin{array}{ll}1.492 & 368 \\
1.486 & 427 \\
1.480 & 293 \\
1.473 & 970 \\
1.467 & 462\end{array}$ & $\begin{array}{ll}-5 & 942 \\
-6 & 134 \\
-6 & 323 \\
-6 & 508 \\
-6 & 689\end{array}$ & $\begin{array}{l}-192 \\
-189 \\
-185 \\
-181 \\
-177\end{array}$ \\
\hline $\begin{array}{l}31 \\
32 \\
33 \\
34 \\
35\end{array}$ & $\begin{array}{ll}1.694 & 114 \\
1.702 & 836 \\
1.711 & 925 \\
1.721 & 391 \\
1.731 & 245\end{array}$ & $\begin{array}{rr}8722 \\
9 & 089 \\
9 & 466 \\
9 & 854 \\
10 & 254 \\
\end{array}$ & $\begin{array}{l}367 \\
377 \\
388 \\
400 \\
412\end{array}$ & $\begin{array}{l}31 \\
32 \\
33 \\
34 \\
35\end{array}$ & $\begin{array}{ll}1.460 & 774 \\
1.453 & 908 \\
1.446 & 869 \\
1.439 & 662 \\
1.432 & 291\end{array}$ & $\begin{array}{ll}-6 & 866 \\
-7 & 039 \\
-7 & 207 \\
47 & 371 \\
-7 & 531\end{array}$ & $\begin{array}{l}-173 \\
-168 \\
-164 \\
-159 \\
-155\end{array}$ \\
\hline $\begin{array}{l}36 \\
37 \\
38 \\
39 \\
40\end{array}$ & $\begin{array}{ll}1.741 & 499 \\
1.752 & 165 \\
1.763 & 256 \\
1.774 & 786 \\
1.786 & 770\end{array}$ & $\begin{array}{ll}10 & 666 \\
11 & 091 \\
11 & 530 \\
11 & 983 \\
12 & 452\end{array}$ & $\begin{array}{l}425 \\
439 \\
453 \\
469 \\
486\end{array}$ & $\begin{array}{l}36 \\
37 \\
38 \\
39 \\
40\end{array}$ & $\begin{array}{ll}1.424 & 760 \\
1.417 & 075 \\
1.409 & 240 \\
1.401 & 260 \\
1.393 & 140\end{array}$ & $\begin{array}{ll}-7 & 685 \\
-7 & 835 \\
-7 & 980 \\
-8 & 120 \\
-8 & 254\end{array}$ & $\begin{array}{l}-150 \\
-145 \\
-140 \\
-134 \\
-129\end{array}$ \\
\hline $\begin{array}{l}1 \\
42 \\
43 \\
44 \\
45\end{array}$ & $\begin{array}{ll}1.799 & 222 \\
1.812 & 160 \\
1.825 & 602 \\
1.839 & 567 \\
1.854 & 075\end{array}$ & $\begin{array}{ll}12 & 938 \\
13 & 442 \\
13 & 965 \\
14 & 508 \\
15 & 073\end{array}$ & $\begin{array}{l}504 \\
523 \\
543 \\
565 \\
588\end{array}$ & $\begin{array}{l}41 \\
42 \\
43 \\
44 \\
45\end{array}$ & 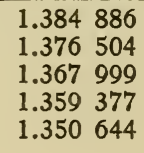 & 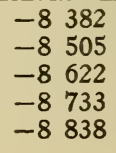 & $\begin{array}{l}-123 \\
-117 \\
-111 \\
-105 \\
-98\end{array}$ \\
\hline
\end{tabular}


TABLE XII-Continued

\begin{tabular}{|c|c|c|c|c|c|c|c|}
\hline & $\mathbf{F}$ & $\Delta_{1}$ & $\triangle_{2}$ & & $\mathbf{E}$ & $\Delta_{1}$ & $\Delta_{2}$ \\
\hline $\begin{array}{l}45^{\circ} \\
46 \\
47 \\
48 \\
49 \\
50\end{array}$ & 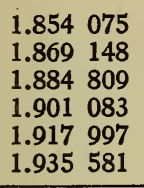 & $\begin{array}{ll}15 & 073 \\
15 & 661 \\
16 & 274 \\
16 & 914 \\
17 & 584 \\
18 & 284 \\
\end{array}$ & $\begin{array}{l}588 \\
613 \\
640 \\
669 \\
700 \\
735 \\
\end{array}$ & $\begin{array}{l}45^{\circ} \\
46 \\
47 \\
48 \\
49 \\
50 \\
\end{array}$ & 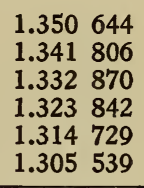 & $\begin{array}{ll}-8 & 838 \\
-8 & 936 \\
-9 & 028 \\
-9 & 113 \\
-9 & 190 \\
-9 & 261\end{array}$ & $\begin{array}{l}-98 \\
-92 \\
-85 \\
-78 \\
-71 \\
-63\end{array}$ \\
\hline $\begin{array}{l}51 \\
52 \\
53 \\
54 \\
55\end{array}$ & $\begin{array}{ll}1.953 & 865 \\
1.972 & 882 \\
1.992 & 670 \\
2.013 & 266 \\
2.034 & 715\end{array}$ & $\begin{array}{ll}19 & 017 \\
19 & 787 \\
20 & 597 \\
21 & 449 \\
22 & 347\end{array}$ & $\begin{array}{l}770 \\
809 \\
852 \\
898 \\
949\end{array}$ & $\begin{array}{l}51 \\
52 \\
53 \\
54 \\
55\end{array}$ & 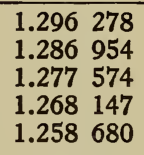 & $\begin{array}{ll}-9 & 324 \\
-9 & 380 \\
-9 & 427 \\
-9 & 467 \\
-9 & 498\end{array}$ & $\begin{array}{l}-56 \\
-48 \\
-40 \\
-31 \\
-22\end{array}$ \\
\hline $\begin{array}{l}56 \\
57 \\
58 \\
59 \\
60\end{array}$ & $\begin{array}{lll}2.057 & 062 \\
2.080 & 358 \\
2.104 & 658 \\
2.130 & 021 \\
2.156 & 516 \\
\end{array}$ & $\begin{array}{ll}23 & 296 \\
24 & 300 \\
25 & 364 \\
26 & 494 \\
27 & 698 \\
\end{array}$ & $\begin{array}{ll}1 & 004 \\
1 & 064 \\
1 & 130 \\
1 & 203 \\
1 & 284 \\
\end{array}$ & $\begin{array}{l}56 \\
57 \\
58 \\
59 \\
60\end{array}$ & $\begin{array}{ll}1.249 & 182 \\
1.239 & 661 \\
1.230 & 127 \\
1.220 & 589 \\
1.211 & 056\end{array}$ & $\begin{array}{ll}-9 & 520 \\
-9 & 534 \\
-9 & 538 \\
-9 & 533 \\
-9 & 518\end{array}$ & $\begin{array}{l}-14 \\
-4 \\
+5 \\
+15 \\
+25\end{array}$ \\
\hline $\begin{array}{l}61 \\
62 \\
63 \\
64 \\
65\end{array}$ & $\begin{array}{ll}2.184 & 213 \\
2.213 & 195 \\
2.243 & 549 \\
2.275 & 376 \\
2.308 & 787\end{array}$ & $\begin{array}{ll}28 & 982 \\
30 & 355 \\
31 & 827 \\
33 & 410 \\
35 & 118\end{array}$ & $\begin{array}{ll}1 & 373 \\
1 & 472 \\
1 & 583 \\
1 & 708 \\
1 & 848\end{array}$ & $\begin{array}{l}61 \\
62 \\
63 \\
64 \\
65\end{array}$ & $\begin{array}{ll}1.201 & 538 \\
1.192 & 046 \\
1.182 & 589 \\
1.173 & 180 \\
1.163 & 828\end{array}$ & $\begin{array}{ll}-9 & 492 \\
-9 & 457 \\
-9 & 410 \\
-9 & 351 \\
-9 & 281\end{array}$ & $\begin{array}{l}36 \\
47 \\
58 \\
70 \\
82\end{array}$ \\
\hline $\begin{array}{l}66 \\
67 \\
68 \\
69 \\
70\end{array}$ & 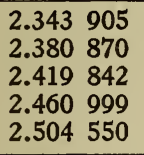 & $\begin{array}{ll}36 & 965 \\
38 & 971 \\
41 & 158 \\
43 & 551 \\
46 & 181 \\
\end{array}$ & $\begin{array}{ll}2 & 006 \\
2 & 186 \\
2 & 393 \\
2 & 631 \\
2 & 907\end{array}$ & $\begin{array}{l}66 \\
67 \\
68 \\
69 \\
70\end{array}$ & $\begin{array}{ll}1.154 & 547 \\
1.145 & 348 \\
1.136 & 244 \\
1.127 & 250 \\
1.118 & 378\end{array}$ & $\begin{array}{ll}-9 & 199 \\
-9 & 104 \\
-8 & 995 \\
-8 & 872 \\
-8 & 734\end{array}$ & $\begin{array}{r}95 \\
109 \\
123 \\
138 \\
153\end{array}$ \\
\hline $\begin{array}{l}71 \\
72 \\
73 \\
74 \\
75\end{array}$ & $\begin{array}{ll}2.550 & 731 \\
2.599 & 820 \\
2.652 & 138 \\
2.708 & 068 \\
2.768 & 063\end{array}$ & $\begin{array}{ll}49 & 088 \\
52 & 318 \\
55 & 930 \\
59 & 996 \\
64 & 609\end{array}$ & $\begin{array}{ll}3 & 230 \\
3 & 611 \\
4 & 066 \\
4 & 614 \\
5 & 283\end{array}$ & $\begin{array}{l}71 \\
72 \\
73 \\
74 \\
75\end{array}$ & $\begin{array}{ll}1.109 & 643 \\
1.101 & 062 \\
1.092 & 650 \\
1.084 & 425 \\
1.076 & 405\end{array}$ & $\begin{array}{ll}-8 & 581 \\
-8 & 412 \\
-8 & 225 \\
-8 & 020 \\
-7 & 796\end{array}$ & $\begin{array}{l}169 \\
187 \\
205 \\
224 \\
245\end{array}$ \\
\hline $\begin{array}{l}76 \\
77 \\
78 \\
79 \\
80\end{array}$ & $\begin{array}{ll}2.832 & 673 \\
2.902 & 565 \\
2.978 & 569 \\
3.061 & 729 \\
3.153 & 385\end{array}$ & $\begin{array}{rr}69 & 892 \\
76 & 004 \\
83 & 160 \\
91 & 657 \\
101 & 918 \\
\end{array}$ & $\begin{array}{rr}6 & 112 \\
7 & 156 \\
8 & 497 \\
10 & 261 \\
12 & 647\end{array}$ & $\begin{array}{l}76 \\
77 . \\
78 \\
79 \\
80\end{array}$ & 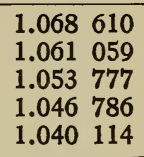 & $\begin{array}{ll}-7 & 550 \\
-7 & 282 \\
-6 & 990 \\
-6 & 672 \\
-6 & 325\end{array}$ & $\begin{array}{l}268 \\
292 \\
318 \\
347 \\
379\end{array}$ \\
\hline $\begin{array}{l}81 \\
82 \\
83 \\
84 \\
85\end{array}$ & $\begin{array}{ll}3.255 & 303 \\
3.369 & 868 \\
3.500 & 422 \\
3.651 & 856 \\
3.831 & 742\end{array}$ & $\begin{array}{ll}114 & 565 \\
130 & 554 \\
151 & 433 \\
179 & 886 \\
221 & 016\end{array}$ & $\begin{array}{ll}15 & 989 \\
20 & 879 \\
28 & 453 \\
41 & 130 \\
64 & 880\end{array}$ & $\begin{array}{l}81 \\
82 \\
83 \\
84 \\
85\end{array}$ & 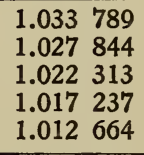 & $\begin{array}{ll}-5 & 946 \\
-5 & 531 \\
-5 & 076 \\
-4 & 573 \\
-4 & 016\end{array}$ & $\begin{array}{l}415 \\
455 \\
502 \\
558 \\
626\end{array}$ \\
\hline $\begin{array}{l}86 \\
87 \\
88 \\
89 \\
90\end{array}$ & 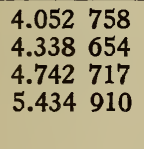 & $\begin{array}{ll}285 & 896 \\
404 & 063 \\
692 & 193\end{array}$ & $\begin{array}{ll}118 & 167 \\
288 & 129\end{array}$ & $\begin{array}{l}86 \\
87 \\
88 \\
89 \\
90\end{array}$ & 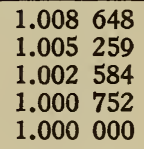 & $\begin{array}{ll}-3 & 389 \\
-2 & 675 \\
-1 & 832 \\
- & 752\end{array}$ & $\begin{array}{r}715 \\
842 \\
1081\end{array}$ \\
\hline
\end{tabular}




\section{TABLE XIII}

Values of $\log F$ and $\log E$

(See Note, p. 213)

\begin{tabular}{|c|c|c|c|c|c|c|}
\hline$\gamma$ & $\log F$ & $\Delta_{1}$ & $\Delta_{2}$ & $\log E$ & $\Delta_{1}$ & $\Delta_{2}$ \\
\hline $\begin{array}{l}45.0 \\
45.1 \\
45.2 \\
45.3 \\
45.4\end{array}$ & $\begin{array}{ll}0.2681 & 2722 \\
0.2684 & 7411 \\
0.2688 & 2204 \\
0.2691 & 7102 \\
0.2695 & 2106 \\
\end{array}$ & $\begin{array}{ll}3 & 4688 \\
3 & 4793 \\
3 & 4898 \\
3 & 5004 \\
3 & 5110 \\
\end{array}$ & $\begin{array}{l}105 \\
105 \\
105 \\
106 \\
106 \\
\end{array}$ & $\begin{array}{l}0.13054086 \\
0.13025807 \\
0.12997476 \\
0.12969094 \\
0.12940659 \\
\end{array}$ & $\begin{array}{ll}2 & 8279 \\
2 & 8331 \\
2 & 8383 \\
2 & 8434 \\
2 & 8486 \\
\end{array}$ & $\begin{array}{l}52 \\
52 \\
52 \\
52 \\
51 \\
\end{array}$ \\
\hline $\begin{array}{l}45.5 \\
45.6 \\
45.7 \\
45.8 \\
45.9\end{array}$ & 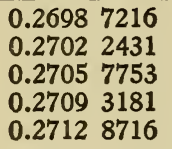 & $\begin{array}{ll}3 & 5216 \\
3 & 5322 \\
3 & 5428 \\
3 & 5535 \\
3 & 5642\end{array}$ & $\begin{array}{l}106 \\
106 \\
107 \\
107 \\
107\end{array}$ & $\begin{array}{ll}0.1291 & 2174 \\
0.1288 & 3636 \\
0.1285 & 5048 \\
0.1282 & 6408 \\
0.1279 & 7717\end{array}$ & $\begin{array}{ll}2 & 8537 \\
2 & 8589 \\
2 & 8640 \\
2 & 8691 \\
2 & 8742\end{array}$ & $\begin{array}{l}51 \\
51 \\
51 \\
51 \\
51\end{array}$ \\
\hline $\begin{array}{l}46.0 \\
46.1 \\
46.2 \\
46.3 \\
46.4\end{array}$ & 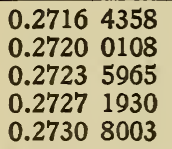 & $\begin{array}{ll}3 & 5749 \\
3 & 5857 \\
3 & 5965 \\
3 & 6073 \\
3 & 6181\end{array}$ & $\begin{array}{l}108 \\
108 \\
108 \\
108 \\
109\end{array}$ & $\begin{array}{ll}0.1276 & 8975 \\
0.1274 & 0182 \\
0.1271 & 1338 \\
0.1268 & 2444 \\
0.1265 & 3499\end{array}$ & $\begin{array}{ll}2 & 8793 \\
2 & 8844 \\
2 & 8894 \\
2 & 8945 \\
2 & 8995\end{array}$ & $\begin{array}{l}51 \\
51 \\
50 \\
50 \\
50\end{array}$ \\
\hline $\begin{array}{l}46.5 \\
46.6 \\
46.7 \\
46.8 \\
46.9\end{array}$ & $\begin{array}{ll}0.2734 & 4184 \\
0.2738 & 0474 \\
0.2741 & 6873 \\
0.2745 & 3381 \\
0.2748 & 9999\end{array}$ & $\begin{array}{ll}3 & 6290 \\
3 & 6399 \\
3 & 6508 \\
3 & 6618 \\
3 & 6728\end{array}$ & $\begin{array}{l}109 \\
109 \\
110 \\
110 \\
110\end{array}$ & 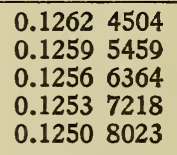 & $\begin{array}{ll}2 & 9045 \\
2 & 9095 \\
2 & 9145 \\
2 & 9195 \\
2 & 9245\end{array}$ & $\begin{array}{l}50 \\
50 \\
50 \\
50 \\
50\end{array}$ \\
\hline $\begin{array}{l}47.0 \\
47.1 \\
47.2 \\
47.3 \\
47.4\end{array}$ & $\begin{array}{lll}0.2752 & 6727 \\
0.2756 & 3565 \\
0.2760 & 0513 \\
0.2763 & 7572 \\
0.2767 & 4741\end{array}$ & $\begin{array}{ll}3 & 6838 \\
3 & 6948 \\
3 & 7059 \\
3 & 7170 \\
3 & 7281\end{array}$ & $\begin{array}{l}110 \\
111 \\
111 \\
111 \\
112\end{array}$ & $\begin{array}{ll}0.1247 & 8778 \\
0.1244 & 9483 \\
0.1242 & 0139 \\
0.1239 & 0746 \\
0.1236 & 1303\end{array}$ & $\begin{array}{ll}2 & 9295 \\
2 & 9344 \\
2 & 9393 \\
2 & 9443 \\
2 & 9492\end{array}$ & $\begin{array}{l}49 \\
49 \\
49 \\
49 \\
49\end{array}$ \\
\hline $\begin{array}{l}47.5 \\
47.6 \\
47.7 \\
47.8 \\
47.9\end{array}$ & $\begin{array}{ll}0.2771 & 2023 \\
0.2774 & 9415 \\
0.2778 & 6920 \\
0.2782 & 4537 \\
0.2786 & 2266\end{array}$ & $\begin{array}{ll}3 & 7393 \\
3 & 7505 \\
3 & 7617 \\
3 & 7729 \\
3 & 7842\end{array}$ & $\begin{array}{l}112 \\
112 \\
112 \\
113 \\
113\end{array}$ & $\begin{array}{ll}0.1233 & 1811 \\
0.1230 & 2271 \\
0.1227 & 2681 \\
0.1224 & 3043 \\
0.1221 & 3357\end{array}$ & $\begin{array}{ll}2 & 9541 \\
2 & 9589 \\
2 & 9638 \\
2 & 9687 \\
2 & 9735\end{array}$ & $\begin{array}{l}49 \\
49 \\
49 \\
48 \\
48\end{array}$ \\
\hline $\begin{array}{l}48.0 \\
48.1 \\
48.2 \\
48.3 \\
48.4\end{array}$ & $\begin{array}{ll}0.2790 & 0109 \\
0.2793 & 8064 \\
0.2797 & 6133 \\
0.2801 & 4315 \\
0.2805 & 2612\end{array}$ & $\begin{array}{ll}3 & 7955 \\
3 & 8069 \\
3 & 8183 \\
3 & 8297 \\
3 & 8411 \\
\end{array}$ & $\begin{array}{l}113 \\
114 \\
114 \\
114 \\
115\end{array}$ & 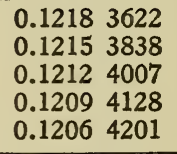 & $\begin{array}{ll}2 & 9783 \\
2 & 9831 \\
2 & 9879 \\
2 & 9927 \\
2 & 9975 \\
\end{array}$ & $\begin{array}{l}48 \\
48 \\
48 \\
48 \\
48 \\
\end{array}$ \\
\hline $\begin{array}{l}48.5 \\
48.6 \\
48.7 \\
48.8 \\
48.9\end{array}$ & $\begin{array}{ll}0.2809 & 1023 \\
0.2812 & 9548 \\
0.2816 & 8189 \\
0.2820 & 6945 \\
0.2824 & 5817\end{array}$ & $\begin{array}{ll}3 & 8526 \\
3 & 8641 \\
3 & 8756 \\
3 & 8872 \\
3 & 8988 \\
\end{array}$ & $\begin{array}{l}115 \\
115 \\
116 \\
116 \\
116\end{array}$ & $\begin{array}{l}0.12034226 \\
0.12004204 \\
0.11974134 \\
0.11944017 \\
0.11913854\end{array}$ & $\begin{array}{ll}3 & 0022 \\
3 & 0070 \\
3 & 0117 \\
3 & 0164 \\
3 & 0211\end{array}$ & $\begin{array}{l}47 \\
47 \\
47 \\
47 \\
47\end{array}$ \\
\hline $\begin{array}{l}49.0 \\
49.1 \\
49.2 \\
49.3 \\
49.4\end{array}$ & $\begin{array}{ll}0.2828 & 4805 \\
0.2832 & 3909 \\
0.2836 & 3130 \\
0.2840 & 2467 \\
0.2844 & 1923\end{array}$ & $\begin{array}{ll}3 & 9104 \\
3 & 9221 \\
3 & 9338 \\
3 & 9455 \\
3 & 9573\end{array}$ & $\begin{array}{l}117 \\
117 \\
117 \\
118 \\
118\end{array}$ & $\begin{array}{ll}0.1188 & 3643 \\
0.1185 & 3385 \\
0.1182 & 3081 \\
0.1179 & 2730 \\
0.1176 & 2333\end{array}$ & $\begin{array}{ll}3 & 0258 \\
3 & 0304 \\
3 & 0351 \\
3 & 0397 \\
3 & 0443\end{array}$ & $\begin{array}{l}47 \\
46 \\
46 \\
46 \\
46\end{array}$ \\
\hline $\begin{array}{l}49.5 \\
49.6 \\
49.7 \\
49.8 \\
49.9 \\
50.0\end{array}$ & $\begin{array}{ll}0.2848 & 1495 \\
0.2852 & 1186 \\
0.2856 & 0996 \\
0.2860 & 0924 \\
0.2864 & 0971 \\
0.2868 & 1137\end{array}$ & $\begin{array}{ll}3 & 9691 \\
3 & 9809 \\
3 & 9928 \\
4 & 0047 \\
4 & 0167 \\
4 & 0286\end{array}$ & $\begin{array}{l}118 \\
119 \\
119 \\
119 \\
120 \\
120\end{array}$ & $\begin{array}{ll}0.1173 & 1890 \\
0.1170 & 1401 \\
0.1167 & 0866 \\
0.1164 & 0286 \\
0.1160 & 9660 \\
0.1157 & 8988\end{array}$ & $\begin{array}{ll}3 & 0489 \\
3 & 0535 \\
3 & 0581 \\
3 & 0626 \\
3 & 0671 \\
3 & 0717\end{array}$ & $\begin{array}{l}46 \\
46 \\
46 \\
45 \\
45 \\
45\end{array}$ \\
\hline
\end{tabular}


TABLE XIII-Continued

\begin{tabular}{|c|c|c|c|c|c|c|}
\hline$\gamma$ & Log $F$ & $\Delta_{1}$ & $\Delta_{2}$ & $\log E$ & $\Delta_{1}$ & $\Delta_{2}$ \\
\hline $\begin{array}{l}50.0 \\
50.1 \\
50.2 \\
50.3 \\
50.4\end{array}$ & 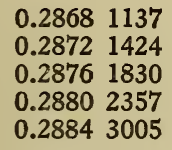 & $\begin{array}{ll}4 & 0286 \\
4 & 0406 \\
4 & 0527 \\
4 & 0648 \\
4 & 0769\end{array}$ & $\begin{array}{l}120 \\
121 \\
121 \\
121 \\
122\end{array}$ & 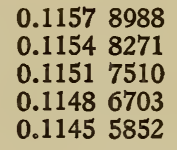 & $\begin{array}{ll}3 & 0717 \\
3 & 0762 \\
3 & 0807 \\
3 & 0851 \\
3 & 0896\end{array}$ & $\begin{array}{l}45 \\
45 \\
45 \\
45 \\
44\end{array}$ \\
\hline $\begin{array}{l}50.5 \\
50.6 \\
50.7 \\
50.8 \\
50.9\end{array}$ & 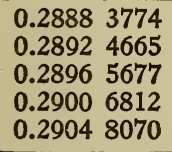 & $\begin{array}{ll}4 & 0891 \\
4 & 1013 \\
4 & 1135 \\
4 & 1258 \\
4 & 1381\end{array}$ & $\begin{array}{l}122 \\
122 \\
123 \\
123 \\
123\end{array}$ & 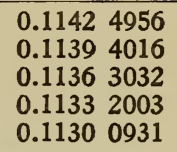 & $\begin{array}{ll}3 & 0940 \\
3 & 0985 \\
3 & 1028 \\
3 & 1072 \\
3 & 1116\end{array}$ & $\begin{array}{l}44 \\
44 \\
44 \\
44 \\
43\end{array}$ \\
\hline $\begin{array}{l}51.0 \\
51.1 \\
51.2 \\
51.3 \\
51.4\end{array}$ & 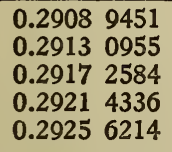 & $\begin{array}{ll}4 & 1504 \\
4 & 1628 \\
4 & 1753 \\
4 & 1877 \\
4 & 2002\end{array}$ & $\begin{array}{l}124 \\
124 \\
125 \\
125 \\
125\end{array}$ & 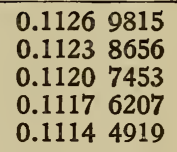 & $\begin{array}{ll}3 & 1159 \\
3 & 1203 \\
3 & 1246 \\
3 & 1289 \\
3 & 1332\end{array}$ & $\begin{array}{l}43 \\
43 \\
43 \\
43 \\
43\end{array}$ \\
\hline $\begin{array}{l}51.5 \\
51.6 \\
51.7 \\
51.8 \\
51.9\end{array}$ & 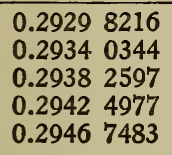 & $\begin{array}{ll}4 & 2128 \\
4 & 2254 \\
4 & 2380 \\
4 & 2506 \\
4 & 2634\end{array}$ & $\begin{array}{l}126 \\
126 \\
127 \\
127 \\
127\end{array}$ & $\begin{array}{ll}0.1111 & 3587 \\
0.1108 & 2213 \\
0.1105 & 0796 \\
0.1101 & 9337 \\
0.1098 & 7836\end{array}$ & $\begin{array}{ll}3 & 1374 \\
3 & 1417 \\
3 & 1459 \\
3 & 1501 \\
3 & 1543\end{array}$ & $\begin{array}{l}42 \\
42 \\
42 \\
42 \\
42\end{array}$ \\
\hline $\begin{array}{l}52.0 \\
52.1 \\
52.2 \\
52.3 \\
52.4\end{array}$ & 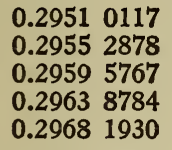 & $\begin{array}{ll}4 & 2761 \\
4 & 2889 \\
4 & 3017 \\
4 & 3146 \\
4 & 3275\end{array}$ & $\begin{array}{l}128 \\
128 \\
129 \\
129 \\
130\end{array}$ & 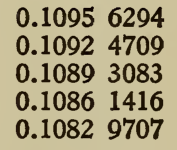 & $\begin{array}{rr}3 & 1584 \\
3 & 1626 \\
3 & 1667 \\
\cdot 3 & 1708 \\
3 & 1749\end{array}$ & $\begin{array}{l}41 \\
41 \\
41 \\
41 \\
41\end{array}$ \\
\hline $\begin{array}{l}52.5 \\
52.6 \\
52.7 \\
52.8 \\
52.9\end{array}$ & 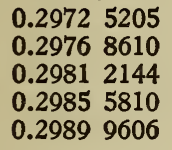 & $\begin{array}{ll}4 & 3405 \\
4 & 3535 \\
4 & 3665 \\
4 & 3796 \\
4 & 3927\end{array}$ & $\begin{array}{l}130 \\
130 \\
131 \\
131 \\
132\end{array}$ & 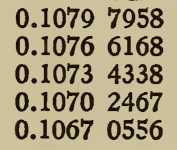 & $\begin{array}{ll}3 & 1790 \\
3 & 1831 \\
3 & 1871 \\
3 & 1911 \\
3 & 1951\end{array}$ & $\begin{array}{l}41 \\
40 \\
40 \\
40 \\
40\end{array}$ \\
\hline $\begin{array}{l}53.0 \\
53.1 \\
53.2 \\
53.3 \\
53.4\end{array}$ & 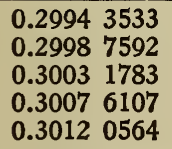 & $\begin{array}{ll}4 & 4059 \\
4 & 4191 \\
4 & 4324 \\
4 & 4457 \\
4 & 4591\end{array}$ & $\begin{array}{l}132 \\
133 \\
133 \\
134 \\
134\end{array}$ & 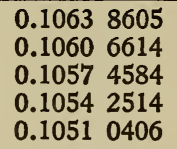 & $\begin{array}{ll}3 & 1991 \\
3 & 2030 \\
3 & 2070 \\
3 & 2109 \\
3 & 2148\end{array}$ & $\begin{array}{l}40 \\
39 \\
39 \\
39 \\
39\end{array}$ \\
\hline $\begin{array}{l}53.5 \\
53.6 \\
53.7 \\
53.8 \\
53.9\end{array}$ & $\begin{array}{l}0.30165155 \\
0.30209880 \\
0.30254739 \\
0.30299733 \\
0.30344863\end{array}$ & $\begin{array}{ll}4 & 4725 \\
4 & 4859 \\
4 & 4994 \\
4 & 5130 \\
4 & 5265\end{array}$ & $\begin{array}{l}134 \\
135 \\
135 \\
136 \\
136\end{array}$ & 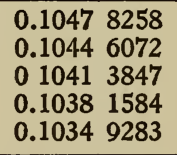 & $\begin{array}{ll}3 & 2186 \\
3 & 2225 \\
3 & 2263 \\
3 & 2301 \\
3 & 2339\end{array}$ & $\begin{array}{l}38 \\
38 \\
38 \\
38 \\
38\end{array}$ \\
\hline $\begin{array}{l}54.0 \\
54.1 \\
54.2 \\
54.3 \\
54.4\end{array}$ & 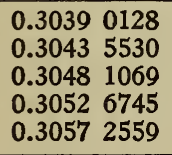 & $\begin{array}{ll}4 & 5402 \\
4 & 5539 \\
4 & 5676 \\
4 & 5814 \\
4 & 5952\end{array}$ & $\begin{array}{l}137 \\
137 \\
138 \\
138 \\
139\end{array}$ & 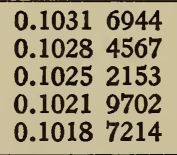 & $\begin{array}{ll}3 & 2377 \\
3 & 2414 \\
3 & 2451 \\
3 & 2488 \\
3 & 2525\end{array}$ & $\begin{array}{l}37 \\
37 \\
37 \\
37 \\
37\end{array}$ \\
\hline $\begin{array}{l}54.5 \\
54.6 \\
54.7 \\
54.8 \\
54.9 \\
55.0\end{array}$ & $\begin{array}{ll}0.3061 & 8511 \\
0.3066 & 4602 \\
0.3071 & 0833 \\
0.3075 & 7203 \\
0.3080 & 3714 \\
0.3085 & 0365\end{array}$ & $\begin{array}{ll}4 & 6091 \\
4 & 6230 \\
4 & 6370 \\
4 & 6511 \\
4 & 6652 \\
4 & 6793\end{array}$ & $\begin{array}{l}139 \\
140 \\
140 \\
141 \\
141 \\
142\end{array}$ & 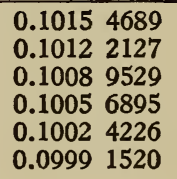 & $\begin{array}{ll}3 & 2562 \\
3 & 2598 \\
3 & 2634 \\
3 & 2670 \\
3 & 2705 \\
3 & 2741\end{array}$ & $\begin{array}{l}36 \\
36 \\
36 \\
36 \\
35 \\
35\end{array}$ \\
\hline
\end{tabular}

$2 \mathrm{I}^{6} 74^{\circ}-\mathrm{I}_{2}-\mathrm{I}_{4}$ 
TABLE XIII-Continued.

\begin{tabular}{|c|c|c|c|c|c|c|}
\hline$\gamma$ & $\log F$ & $\Delta_{1}$ & $\triangle_{2}$ & $\log E$ & $\Delta_{1}$ & $\triangle_{2}$ \\
\hline $\begin{array}{l}55: 0 \\
55.1 \\
55.2 \\
55.3 \\
55.4\end{array}$ & 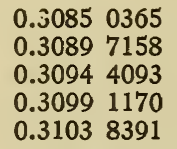 & $\begin{array}{ll}4 & 6793 \\
4 & 6935 \\
4 & 7077 \\
4 & 7220 \\
4 & 7364\end{array}$ & $\begin{array}{l}142 \\
142 \\
143 \\
143 \\
144\end{array}$ & $\begin{array}{ll}0.0999 & 1520 \\
0.0995 & 8779 \\
0.0992 & 6003 \\
0.0989 & 3193 \\
0.0986 & 0347\end{array}$ & $\begin{array}{ll}3 & 2741 \\
3 & 2776 \\
3 & 2811 \\
3 & 2846 \\
3 & 2880\end{array}$ & $\begin{array}{l}35 \\
35 \\
35 \\
34 \\
34\end{array}$ \\
\hline $\begin{array}{l}55.5 \\
55.6 \\
55.7 \\
55.8 \\
55.9\end{array}$ & $\begin{array}{ll}0.3108 & 5754 \\
0.3113 & 3262 \\
0.3118 & 0915 \\
0.3122 & 8712 \\
0.3127 & 6655\end{array}$ & $\begin{array}{ll}4 & 7508 \\
4 & 7652 \\
4 & 7798 \\
4 & 7943 \\
4 & 8089\end{array}$ & $\begin{array}{l}145 \\
145 \\
146 \\
146 \\
147\end{array}$ & 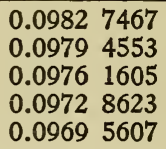 & $\begin{array}{ll}3 & 2914 \\
3 & 2948 \\
3 & 2982 \\
3 & 3015 \\
3 & 3049\end{array}$ & $\begin{array}{l}34 \\
34 \\
33 \\
33 \\
33\end{array}$ \\
\hline $\begin{array}{l}56.0 \\
56.1 \\
56.2 \\
56.3 \\
56.4\end{array}$ & $\begin{array}{ll}0.3132 & 4745 \\
0.3137 & 2981 \\
0.3142 & 1365 \\
0.3146 & 9896 \\
0.3151 & 8577\end{array}$ & $\begin{array}{ll}4 & 8236 \\
4 & 8384 \\
4 & 8532 \\
48680 \\
4 & 8829\end{array}$ & $\begin{array}{l}147 \\
148 \\
149 \\
149 \\
150\end{array}$ & $\begin{array}{l}0.09662559 \\
0.09629477 \\
0.09596363 \\
0.09563216 \\
0.09530037\end{array}$ & $\begin{array}{ll}3 & 3082 \\
3 & 3114 \\
3 & 3147 \\
3 & 3179 \\
3 & 3211\end{array}$ & $\begin{array}{l}33 \\
32 \\
32 \\
32 \\
32\end{array}$ \\
\hline $\begin{array}{l}56.5 \\
56.6 \\
56.7 \\
56.8 \\
56.9\end{array}$ & $\begin{array}{ll}0.3156 & 7406 \\
0.3161 & 6385 \\
0.3166 & 5514 \\
0.3171 & 4794 \\
0.3176 & 4226\end{array}$ & $\begin{array}{ll}4 & 8979 \\
4 & 9129 \\
4 & 9280 \\
4 & 9432 \\
4 & 9584\end{array}$ & $\begin{array}{l}150 \\
151 \\
151 \\
152 \\
153\end{array}$ & $\begin{array}{l}0.09496826 \\
0.09463583 \\
0.09430309 \\
0.09397003 \\
0.09363667\end{array}$ & $\begin{array}{ll}3 & 3243 \\
3 & 3274 \\
3 & 3305 \\
3 & 3336 \\
3 & 3367\end{array}$ & $\begin{array}{l}31 \\
31 \\
31 \\
31 \\
30\end{array}$ \\
\hline $\begin{array}{l}57.0 \\
57.1 \\
57.2 \\
57.3 \\
57.4 \\
\end{array}$ & $\begin{array}{lll}0.3181 & 3809 \\
0.3186 & 3545 \\
0.3191 & 3435 \\
0.3196 & 3479 \\
0.3201 & 3677 \\
\end{array}$ & $\begin{array}{ll}4 & 9736 \\
4 & 9890 \\
5 & 0044 \\
5 & 0198 \\
5 & 0353 \\
\end{array}$ & $\begin{array}{l}153 \\
154 \\
155 \\
155 \\
156\end{array}$ & $\begin{array}{l}0.09330300 \\
0.09296903 \\
0.09263475 \\
0.09230018 \\
0.09196531\end{array}$ & $\begin{array}{ll}3 & 3397 \\
3 & 3428 \\
3 & 3457 \\
3 & 3487 \\
3 & 3516\end{array}$ & $\begin{array}{l}30 \\
30 \\
30 \\
29 \\
29\end{array}$ \\
\hline $\begin{array}{l}57.5 \\
57.6 \\
57.7 \\
57.8 \\
57.9\end{array}$ & $\begin{array}{ll}0.3206 & 4030 \\
0.3211 & 4539 \\
0.3216 & 5204 \\
0.3221 & 6027 \\
0.3226 & 7008\end{array}$ & $\begin{array}{ll}5 & 0509 \\
5 & 0666 \\
5 & 0823 \\
5 & 0980 \\
5 & 1139\end{array}$ & $\begin{array}{l}156 \\
157 \\
158 \\
158 \\
159\end{array}$ & 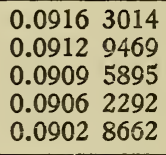 & $\begin{array}{ll}3 & 3545 \\
3 & 3574 \\
3 & 3603 \\
3 & 3631 \\
3 & 3659\end{array}$ & $\begin{array}{l}29 \\
28 \\
28 \\
28 \\
28\end{array}$ \\
\hline $\begin{array}{l}58.0 \\
58.1 \\
58.2 \\
58.3 \\
58.4\end{array}$ & $\begin{array}{ll}0.3231 & 8146 \\
0.3236 & 9444 \\
0.3242 & 0902 \\
0.3247 & 2520 \\
0.3252 & 4299\end{array}$ & $\begin{array}{ll}5 & 1298 \\
5 & 1458 \\
5 & 1618 \\
5 & 1779 \\
5 & 1941\end{array}$ & $\begin{array}{l}160 \\
160 \\
161 \\
162 \\
162\end{array}$ & $\begin{array}{ll}0.0899 & 5003 \\
0.0896 & 1317 \\
0.0892 & 7603 \\
0.0889 & 3862 \\
0.0886 & 0095\end{array}$ & $\begin{array}{ll}3 & 3686 \\
3 & 3714 \\
3 & 3741 \\
3 & 3767 \\
3 & 3794\end{array}$ & $\begin{array}{l}27 \\
27 \\
27 \\
26 \\
26\end{array}$ \\
\hline $\begin{array}{l}58.5 \\
58.6 \\
58.7 \\
58.8 \\
58.9 \\
\end{array}$ & 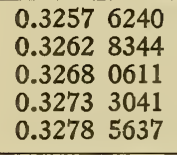 & $\begin{array}{ll}5 & 2104 \\
5 & 2267 \\
5 & 2431 \\
5 & 2595 \\
5 & 2761 \\
\end{array}$ & $\begin{array}{l}163 \\
164 \\
165 \\
165 \\
166\end{array}$ & 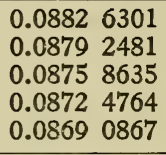 & $\begin{array}{ll}3 & 3820 \\
3 & 3846 \\
3 & 3871 \\
3 & 3897 \\
3 & 3922 \\
\end{array}$ & $\begin{array}{l}26 \\
26 \\
25 \\
25 \\
25 \\
\end{array}$ \\
\hline $\begin{array}{l}59.0 \\
59.1 \\
59.2 \\
59.3 \\
59.4\end{array}$ & $\begin{array}{ll}0.3283 & 8397 \\
0.3289 & 1324 \\
0.3294 & 4418 \\
0.3299 & 7679 \\
0.3305 & 1108\end{array}$ & $\begin{array}{ll}5 & 2927 \\
5 & 3094 \\
5 & 3261 \\
5 & 3429 \\
5 & 3598\end{array}$ & $\begin{array}{l}167 \\
168 \\
168 \\
169 \\
170\end{array}$ & $\begin{array}{ll}0.0865 & 6945 \\
0.0862 & 2999 \\
0.0858 & 9028 \\
0.0855 & 5033 \\
0.0852 & 1015\end{array}$ & $\begin{array}{ll}3 & 3946 \\
3 & 3971 \\
3 & 3995 \\
3 & 4018 \\
3 & 4042\end{array}$ & $\begin{array}{l}24 \\
24 \\
24 \\
23 \\
23\end{array}$ \\
\hline $\begin{array}{l}59.5 \\
59.6 \\
59.7 \\
59.8 \\
59.9 \\
60.0\end{array}$ & $\begin{array}{ll}0.3310 & 4707 \\
0.3315 & 8475 \\
0.3321 & 2414 \\
0.3326 & 6524 \\
0.3332 & 0806 \\
0.3337 & 5261\end{array}$ & $\begin{array}{ll}5 & 3768 \\
5 & 3939 \\
5 & 4110 \\
5 & 4282 \\
5 & 4455 \\
5 & 4629\end{array}$ & $\begin{array}{l}171 \\
171 \\
172 \\
173 \\
174 \\
175\end{array}$ & $\begin{array}{ll}0.0848 & 6973 \\
0.0845 & 2908 \\
0.0841 & 8820 \\
0.0838 & 4710 \\
0.0835 & 0578 \\
0.0831 & 6424\end{array}$ & $\begin{array}{ll}3 & 4065 \\
3 & 4088 \\
3 & 4110 \\
3 & 4132 \\
3 & 4154 \\
3 & 4176\end{array}$ & $\begin{array}{l}23 \\
22 \\
22 \\
22 \\
21 \\
21\end{array}$ \\
\hline
\end{tabular}


TABLE XIII-Continued

\begin{tabular}{|c|c|c|c|c|c|c|}
\hline$\gamma$ & $\log F$ & $\Delta_{1}$ & $\Delta_{2}$ & $\log E$ & $\Delta_{1}$ & $\Delta_{2}$ \\
\hline $60: 0$ & 0.33375261 & 54629 & 175 & 0.08316424 & 34176 & 21 \\
\hline 60.1 & 0.33429890 & 54803 & 175 & $0.0828 \quad 2248$ & 34197 & 21 \\
\hline 60.2 & 0.33484694 & 54979 & 176 & 0.08248051 & 34217 & 20 \\
\hline 60.3 & 0.33539673 & 55155 & 177 & 0.08213834 & 34238 & 20 \\
\hline 60.4 & 0.33594827 & 55332 & 178 & 0.08179596 & 34258 & 20 \\
\hline 60.5 & 0.33650159 & 55510 & 179 & 0.08145338 & 34278 & 19 \\
\hline 60.6 & 0.33705669 & 55688 & 179 & 0.08111060 & 34297 & 19 \\
\hline 60.7 & 0.33761357 & 55868 & 180 & 0.08076763 & 34316 & 19 \\
\hline 60.8 & 0.33817225 & 56048 & 181 & 0.08042446 & 34335 & 18 \\
\hline 60.9 & 0.33873274 & 56229 & 182 & 0.08008111 & 34354 & 18 \\
\hline 61.0 & 0.33929503 & 56412 & 183 & 0.07973758 & 34372 & 18 \\
\hline 61.1 & 0.33985915 & 56595 & 184 & 0.07939386 & 34389 & 17 \\
\hline 61.2 & 0.34042509 & 56778 & 185 & 0.07904997 & 34407 & 17 \\
\hline 61.3 & 0.34099288 & 56963 & 186 & 0.07870590 & 34424 & 17 \\
\hline 61.4 & 0.34156251 & 57149 & 187 & 0.07836167 & 34440 & 16 \\
\hline 61.5 & 0.34213400 & 57336 & 188 & $0.0780 \quad 1727$ & 34456 & 16 \\
\hline 61.6 & 0.34270735 & 57523 & 188 & 0.07767270 & 34472 & 15 \\
\hline 61.7 & 0.34328258 & 57712 & 189 & 0.07732798 & 34488 & 15 \\
\hline 61.8 & 0.34385970 & 57901 & 190 & 0.07698310 & 34503 & 15 \\
\hline 61.9 & 0.34443871 & 58091 & 191 & 0.07663807 & 34518 & 14 \\
\hline 62.0 & $0.3450 \quad 1962$ & 58283 & 192 & 0.07629290 & 34532 & 14 \\
\hline 62.1 & 0.34560245 & 58475 & 193 & 0.07594758 & 34546 & 14 \\
\hline 62.2 & 0.34618720 & 58668 & 194 & 0.07560212 & 34560 & 13 \\
\hline 62.3 & 0.34677388 & 58863 & 195 & 0.07525652 & 34573 & 13 \\
\hline 62.4 & 0.34736250 & 59058 & 196 & 1.07491079 & 34586 & 12 \\
\hline 62.5 & 0.34795308 & 59254 & 197 & 0.07456494 & 34598 & 12 \\
\hline 62.6 & 0.34854562 & 59451 & 198 & $0.0742 \quad 1895$ & 34610 & 12 \\
\hline 62.7 & 0.34914014 & 59650 & 199 & 0.07387285 & 34622 & 11 \\
\hline 62.8 & 0.34973664 & 59849 & 200 & 0.07352664 & 34633 & 11 \\
\hline 62.9 & 0.35033513 & 60050 & 202 & 0.07318030 & 34644 & 10 \\
\hline 63.0 & 0.35093563 & 60251 & 203 & 0.07283387 & 34654 & 10 \\
\hline 63.1 & 0.35153814 & 60454 & 204 & 0.07248732 & 34664 & 10 \\
\hline 63.2 & 0.35214268 & 60658 & 205 & 0.07214068 & 34674 & 9 \\
\hline 63.3 & 0.35274925 & 60862 & 206 & 0.07179394 & 34683 & 9 \\
\hline 63.4 & 0.35335787 & 61068 & 207 & 0.07144711 & 34692 & 8 \\
\hline 63.5 & 0.35396856 & 61275 & 208 & 0.07110019 & 34700 & 8 \\
\hline 63.6 & 0.35458131 & 61483 & 209 & 0.07075319 & 34708 & 8 \\
\hline 63.7 & 0.35519614 & 61693 & 210 & 0.07040610 & 34716 & 7 \\
\hline 63.8 & $0.3558 \quad 1307$ & 61903 & 212 & 0.07005895 & 34723 & 7 \\
\hline 63.9 & 0.35643211 & 62115 & 213 & 0.06971172 & 34729 & 6 \\
\hline 64.0 & $0.3570 \quad 5325$ & 62328 & 214 & 0.06936442 & 34736 & 6 \\
\hline 64.1 & 0.35767653 & 62542 & 215 & 0.06901706 & 34741 & 5 \\
\hline 64.2 & 0.35830195 & 62757 & 216 & 0.06866965 & 34747 & 5 \\
\hline 64.3 & 0.35892952 & 62974 & 218 & 0.06832218 & 34752 & 4 \\
\hline 64.4 & 0.35955926 & 63191 & 219 & 0.06797466 & 34756 & 4 \\
\hline 64.5 & 0.36019117 & 63410 & 220 & 0.06762710 & 34760 & 4 \\
\hline 64.6 & 0.36082527 & 63630 & 221 & 0.06727950 & 34764 & 3 \\
\hline 64.7 & 0.36146158 & 63852 & 223 & 0.06693186 & 34767 & 3 \\
\hline 64.8 & 0.36210009 & 64075 & 224 & 0.06658420 & 34769 & 2 \\
\hline 64.9 & 0.36274084 & 64299 & 225 & 0.06623650 & 34772 & 2 \\
\hline 65.0 & 0.36338383 & 64524 & 227 & 0.06588879 & 34773 & 1 \\
\hline
\end{tabular}


TABLE XIII-Continued

\begin{tabular}{|c|c|c|c|c|c|c|}
\hline$\gamma$ & $\log F$ & $\triangle_{1}$ & $\triangle_{2}$ & $\log E$ & $\triangle_{1}$ & $\triangle_{2}$ \\
\hline $\begin{array}{l}65.0 \\
65.1 \\
65.2 \\
65.3 \\
65.4 \\
\end{array}$ & 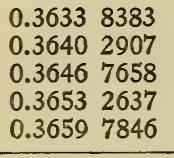 & $\begin{array}{ll}6 & 4524 \\
6 & 4751 \\
6 & 4979 \\
6 & 5209 \\
6 & 5439 \\
\end{array}$ & $\begin{array}{l}227 \\
228 \\
229 \\
231 \\
232 \\
\end{array}$ & 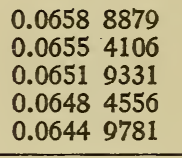 & $\begin{array}{ll}3 & 4773 \\
3 & 4774 \\
3 & 4775 \\
3 & 4775 \\
3 & 4775\end{array}$ & $\begin{array}{r}1 \\
1 \\
+0 \\
-0 \\
1\end{array}$ \\
\hline $\begin{array}{l}65.5 \\
65.6 \\
65.7 \\
65.8 \\
65.9\end{array}$ & $\begin{array}{ll}0.3666 & 3286 \\
0.3672 & 8957 \\
0.3679 & 4863 \\
0.3686 & 1003 \\
0.3692 & 7380 \\
\end{array}$ & $\begin{array}{ll}6 & 5672 \\
6 & 5905 \\
6 & 6141 \\
6 & 6377 \\
6 & 6615 \\
\end{array}$ & $\begin{array}{l}234 \\
235 \\
237 \\
238 \\
239 \\
\end{array}$ & $\begin{array}{ll}0.0641 & 5005 \\
0.0638 & 0231 \\
0.0634 & 5457 \\
0.0631 & 0686 \\
0.0627 & 5916 \\
\end{array}$ & $\begin{array}{ll}3 & 4775 \\
3 & 4773 \\
3 & 4772 \\
3 & 4769 \\
3 & 4767 \\
\end{array}$ & $\begin{array}{l}1 \\
2 \\
2 \\
3 \\
3 \\
\end{array}$ \\
\hline $\begin{array}{l}66.0 \\
66.1 \\
66.2 \\
66.3 \\
66.4\end{array}$ & 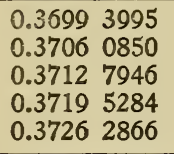 & $\begin{array}{ll}6 & 6855 \\
6 & 7096 \\
6 & 7338 \\
6 & 7582 \\
6 & 7828\end{array}$ & $\begin{array}{l}241 \\
242 \\
244 \\
246 \\
247\end{array}$ & $\begin{array}{ll}0.0624 & 1150 \\
0.0620 & 6386 \\
0.0617 & 1626 \\
0.0613 & 6870 \\
0.0610 & 2119\end{array}$ & $\begin{array}{ll}3 & 4764 \\
3 & 4760 \\
3 & 4756 \\
3 & 4751 \\
3 & 4746\end{array}$ & $\begin{array}{l}4 \\
4 \\
5 \\
5 \\
6\end{array}$ \\
\hline $\begin{array}{l}66.5 \\
66.6 \\
66.7 \\
66.8 \\
66.9 \\
\end{array}$ & 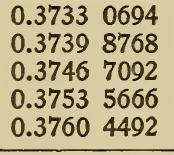 & 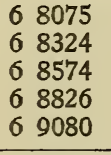 & $\begin{array}{l}249 \\
250 \\
252 \\
254 \\
255 \\
\end{array}$ & $\begin{array}{l}0.06067373 \\
0.06032633 \\
0.05997899 \\
0.0596 \quad 31 ' 72 \\
0.05928453 \\
\end{array}$ & $\begin{array}{ll}3 & 4740 \\
3 & 4734 \\
3 & 4727 \\
3 & 4720 \\
3 & 4712 \\
\end{array}$ & $\begin{array}{l}6 \\
7 \\
7 \\
8 \\
8 \\
\end{array}$ \\
\hline $\begin{array}{l}67.0 \\
67.1 \\
67.2 \\
67.3 \\
67.4 \\
\end{array}$ & $\begin{array}{ll}0.3767 & 3572 \\
0.3774 & 2907 \\
0.3781 & 2499 \\
0.3788 & 2349 \\
0.3795 & 2460 \\
\end{array}$ & $\begin{array}{ll}6 & 9335 \\
6 & 9592 \\
6 & 9851 \\
7 & 0111 \\
7 & 0373 \\
\end{array}$ & $\begin{array}{l}257 \\
259 \\
260 \\
262 \\
264 \\
\end{array}$ & 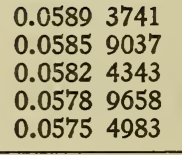 & $\begin{array}{ll}3 & 4703 \\
3 & 4695 \\
3 & 4685 \\
3 & 4675 \\
3 & 4664 \\
\end{array}$ & $\begin{array}{r}9 \\
9 \\
10 \\
11 \\
11 \\
\end{array}$ \\
\hline $\begin{array}{l}67.5 \\
67.6 \\
67.7 \\
67.8 \\
67.9 \\
\end{array}$ & $\begin{array}{ll}0.3802 & 2833 \\
0.3809 & 3471 \\
0.3816 & 4373 \\
0.3823 & 5544 \\
0.3830 & 6984 \\
\end{array}$ & $\begin{array}{ll}7 & 0637 \\
7 & 0903 \\
7 & 1170 \\
7 & 1440 \\
7 & 1711 \\
\end{array}$ & $\begin{array}{l}266 \\
268 \\
269 \\
271 \\
273 \\
\end{array}$ & $\begin{array}{ll}0.0572 & 0318 \\
0.0568 & 5665 \\
0.0565 & 1023 \\
0.0561 & 6394 \\
0.0558 & 1777 \\
\end{array}$ & $\begin{array}{ll}3 & 4653 \\
3 & 4642 \\
3 & 4629 \\
3 & 4617 \\
3 & 4603 \\
\end{array}$ & $\begin{array}{l}12 \\
12 \\
13 \\
13 \\
14 \\
\end{array}$ \\
\hline $\begin{array}{l}68.0 \\
68.1 \\
68.2 \\
68.3 \\
68.4\end{array}$ & 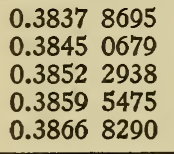 & $\begin{array}{ll}7 & 1984 \\
7 & 2259 \\
7 & 2536 \\
7 & 2815 \\
7 & 3096 \\
\end{array}$ & $\begin{array}{l}275 \\
277 \\
279 \\
281 \\
283\end{array}$ & $\begin{array}{ll}0.0554 & 7174 \\
0.0551 & 2585 \\
0.0547 & 8011 \\
0.0544 & 3451 \\
0.0540 & 8908\end{array}$ & $\begin{array}{ll}3 & 4589 \\
3 & 4575 \\
3 & 4559 \\
3 & 4544 \\
3 & 4527\end{array}$ & $\begin{array}{l}15 \\
15 \\
16 \\
16 \\
17\end{array}$ \\
\hline $\begin{array}{l}68.5 \\
68.6 \\
68.7 \\
68.8 \\
68.9 \\
\end{array}$ & $\begin{array}{ll}0.3874 & 1386 \\
0.3881 & 4765 \\
0.3888 & 8429 \\
0.3896 & 2380 \\
0.3903 & 6620 \\
\end{array}$ & $\begin{array}{ll}7 & 3379 \\
7 & 3664 \\
7 & 3951 \\
7 & 4240 \\
7 & 4531 \\
\end{array}$ & $\begin{array}{l}285 \\
287 \\
289 \\
291 \\
293 \\
\end{array}$ & 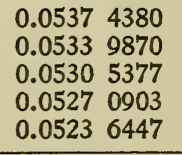 & $\begin{array}{ll}3 & 4510 \\
3 & 4493 \\
3 & 4475 \\
3 & 4456 \\
3 & 4436 \\
\end{array}$ & $\begin{array}{l}18 \\
18 \\
19 \\
19 \\
20 \\
\end{array}$ \\
\hline $\begin{array}{l}69.0 \\
69.1 \\
69.2 \\
69.3 \\
69.4 \\
\end{array}$ & $\begin{array}{ll}0.3911 & 1152 \\
0.3918 & 5977 \\
0.3926 & 1097 \\
0.3933 & 6515 \\
0.3941 & 2234 \\
\end{array}$ & $\begin{array}{ll}7 & 4825 \\
7 & 5120 \\
7 & 5418 \\
7 & 5718 \\
7 & 6020 \\
\end{array}$ & $\begin{array}{l}296 \\
298 \\
300 \\
302 \\
305 \\
\end{array}$ & 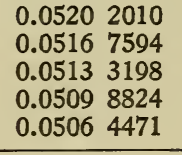 & $\begin{array}{ll}3 & 4416 \\
3 & 4396 \\
3 & 4375 \\
3 & 4353 \\
3 & 4330 \\
\end{array}$ & $\begin{array}{l}21 \\
21 \\
22 \\
23 \\
23 \\
\end{array}$ \\
\hline $\begin{array}{l}69.5 \\
69.6 \\
69.7 \\
69.8 \\
69.9 \\
70.0\end{array}$ & 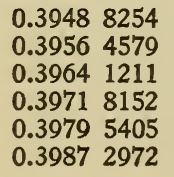 & $\begin{array}{ll}7 & 6325 \\
7 & 6632 \\
7 & 6941 \\
7 & 7253 \\
7 & 7567 \\
7 & 7883\end{array}$ & $\begin{array}{l}307 \\
309 \\
312 \\
314 \\
317 \\
319\end{array}$ & $\begin{array}{ll}0.0503 & 0141 \\
0.0499 & 5834 \\
0.0496 & 1551 \\
0.0492 & 7292 \\
0.0489 & 3059 \\
0.0485 & 8851\end{array}$ & $\begin{array}{ll}3 & 4307 \\
3 & 4283 \\
3 & 4259 \\
3 & 4233 \\
3 & 4208 \\
3 & 4181\end{array}$ & $\begin{array}{l}24 \\
24 \\
25 \\
26 \\
26 \\
27\end{array}$ \\
\hline
\end{tabular}


TABLE XIII-Continued

\begin{tabular}{|c|c|c|c|c|c|c|}
\hline$\gamma$ & Log $F$ & $\triangle_{1}$ & $\Delta_{2}$ & $\log E$ & $\Delta_{1}$ & $\triangle_{2}$ \\
\hline $\begin{array}{l}70: 0 \\
70.1 \\
70.2 \\
70.3 \\
70.4 \\
\end{array}$ & $\begin{array}{ll}0.3987 & 2972 \\
0.3995 & 0855 \\
0.4002 & 9058 \\
0.4010 & 7582 \\
0.4018 & 6430 \\
\end{array}$ & $\begin{array}{ll}7 & 7883 \\
7 & 8202 \\
7 & 8524 \\
7 & 8848 \\
7 & 9175 \\
\end{array}$ & $\begin{array}{l}319 \\
322 \\
324 \\
327 \\
329 \\
\end{array}$ & 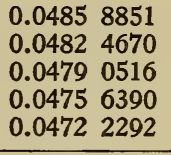 & $\begin{array}{ll}3 & 4181 \\
3 & 4154 \\
3 & 4126 \\
3 & 4098 \\
3 & 4068 \\
\end{array}$ & $\begin{array}{l}27 \\
28 \\
29 \\
29 \\
30 \\
\end{array}$ \\
\hline $\begin{array}{l}70.5 \\
70.6 \\
70.7 \\
70.8 \\
70.9 \\
\end{array}$ & $\begin{array}{ll}0.4026 & 5605 \\
0.4034 & 5109 \\
0.4042 & 4945 \\
0.4050 & 5116 \\
0.4058 & 5625 \\
\end{array}$ & $\begin{array}{ll}7 & 9504 \\
7 & 9836 \\
8 & 0171 \\
8 & 0508 \\
8 & 0849 \\
\end{array}$ & $\begin{array}{l}332 \\
335 \\
337 \\
340 \\
343 \\
\end{array}$ & 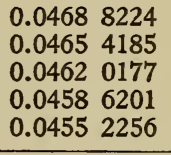 & $\begin{array}{ll}3 & 4039 \\
3 & 4008 \\
3 & 3977 \\
3 & 3945 \\
3 & 3912 \\
\end{array}$ & $\begin{array}{l}31 \\
31 \\
32 \\
33 \\
33 \\
\end{array}$ \\
\hline $\begin{array}{l}71.0 \\
71.1 \\
71.2 \\
71.3 \\
71.4 \\
\end{array}$ & 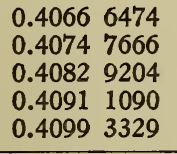 & $\begin{array}{ll}8 & 1192 \\
8 & 1538 \\
8 & 1887 \\
8 & 2239 \\
8 & 2594 \\
\end{array}$ & $\begin{array}{l}346 \\
349 \\
352 \\
355 \\
358 \\
\end{array}$ & $\begin{array}{ll}0.0451 & 8344 \\
0.0448 & 4465 \\
0.0445 & 0621 \\
0.0441 & 6812 \\
0.0438 & 3038 \\
\end{array}$ & $\begin{array}{ll}3 & 3879 \\
3 & 3844 \\
3 & 3810 \\
3 & 3774 \\
3 & 3738 \\
\end{array}$ & $\begin{array}{l}34 \\
35 \\
36 \\
36 \\
37\end{array}$ \\
\hline $\begin{array}{l}71.5 \\
71.6 \\
71.7 \\
71.8 \\
71.9 \\
\end{array}$ & $\begin{array}{ll}0.4107 & 5923 \\
0.4115 & 8875 \\
0.4124 & 2187 \\
0.4132 & 5864 \\
0.4140 & 9909 \\
\end{array}$ & $\begin{array}{ll}8 & 2952 \\
8 & 3313 \\
8 & 3677 \\
8 & 4044 \\
8 & 4415 \\
\end{array}$ & $\begin{array}{l}361 \\
364 \\
367 \\
371 \\
374 \\
\end{array}$ & $\begin{array}{l}0.04349300 \\
0.04315600 \\
0.04281937 \\
0.04248313 \\
0.04214729 \\
\end{array}$ & $\begin{array}{ll}3 & 3700 \\
3 & 3663 \\
3 & 3624 \\
3 & 3585 \\
3 & 3544 \\
\end{array}$ & $\begin{array}{l}38 \\
39 \\
39 \\
40 \\
41\end{array}$ \\
\hline $\begin{array}{l}72.0 \\
72.1 \\
72.2 \\
72.3 \\
72.4\end{array}$ & 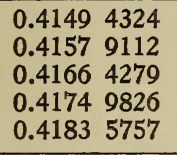 & $\begin{array}{ll}8 & 4789 \\
8 & 5166 \\
8 & 5547 \\
8 & 5931 \\
8 & 6319 \\
\end{array}$ & $\begin{array}{l}377 \\
381 \\
384 \\
388 \\
391\end{array}$ & 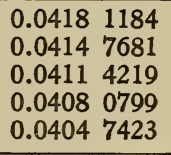 & $\begin{array}{ll}3 & 3504 \\
3 & 3462 \\
3 & 3419 \\
3 & 3376 \\
3 & 3332\end{array}$ & $\begin{array}{l}42 \\
42 \\
43 \\
44 \\
45\end{array}$ \\
\hline $\begin{array}{l}72.5 \\
72.6 \\
72.7 \\
72.8 \\
72.9\end{array}$ & $\begin{array}{ll}0.4192 & 2076 \\
0.4200 & 8786 \\
0.4209 & 5891 \\
0.4218 & 3394 \\
0.4227 & 1300\end{array}$ & $\begin{array}{ll}8 & 6710 \\
8 & 7105 \\
8 & 7503 \\
8 & 7906 \\
8 & 8312\end{array}$ & $\begin{array}{l}395 \\
399 \\
402 \\
406 \\
410\end{array}$ & $\begin{array}{ll}0.0401 & 4091 \\
0.0398 & 0804 \\
0.0394 & 7563 \\
0.0391 & 4368 \\
0.0388 & 1220\end{array}$ & $\begin{array}{ll}3 & 3287 \\
3 & 3241 \\
3 & 3195 \\
3 & 3148 \\
3 & 3099\end{array}$ & $\begin{array}{l}46 \\
46 \\
47 \\
48 \\
49\end{array}$ \\
\hline $\begin{array}{l}73.0 \\
73.1 \\
73.2 \\
73.3 \\
73.4\end{array}$ & $\begin{array}{l}0.42359612 \\
0.42448334 \\
0.4253 \quad 7470 \\
0.4262 \quad 7023 \\
0.42716999\end{array}$ & $\begin{array}{ll}8 & 8722 \\
8 & 9136 \\
8 & 9554 \\
8 & 9976 \\
9 & 0402\end{array}$ & $\begin{array}{l}414 \\
418 \\
422 \\
426 \\
430\end{array}$ & 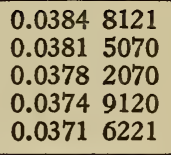 & $\begin{array}{ll}3 & 3050 \\
3 & 3001 \\
3 & 2950 \\
3 & 2898 \\
3 & 2846\end{array}$ & $\begin{array}{l}50 \\
51 \\
52 \\
52 \\
53\end{array}$ \\
\hline $\begin{array}{l}73.5 \\
73.6 \\
73.7 \\
73.8 \\
73.9\end{array}$ & 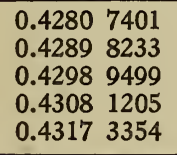 & $\begin{array}{ll}9 & 0832 \\
9 & 1267 \\
9 & 1706 \\
9 & 2149 \\
9 & 2597\end{array}$ & $\begin{array}{l}435 \\
439 \\
443 \\
448 \\
452\end{array}$ & $\begin{array}{ll}0.0368 & 3375 \\
0.0365 & 0582 \\
0.0361 & 7843 \\
0.0358 & 5160 \\
0.0355 & 2532\end{array}$ & $\begin{array}{ll}3 & 2793 \\
3 & 2739 \\
3 & 2684 \\
3 & 2628 \\
3 & 2571 \\
\end{array}$ & $\begin{array}{l}54 \\
55 \\
56 \\
57 \\
58\end{array}$ \\
\hline $\begin{array}{l}74.0 \\
74.1 \\
74.2 \\
74.3 \\
74.4\end{array}$ & 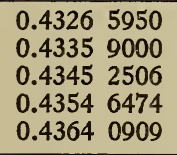 & $\begin{array}{ll}9 & 3049 \\
9 & 3506 \\
9 & 3968 \\
9 & 4435 \\
9 & 4906\end{array}$ & $\begin{array}{l}457 \\
462 \\
467 \\
472 \\
477\end{array}$ & $\begin{array}{ll}0.0351 & 9961 \\
0.0348 & 7448 \\
0.0345 & 4993 \\
0.0342 & 2598 \\
0.0339 & 0263\end{array}$ & $\begin{array}{ll}3 & 2513 \\
3 & 2455 \\
3 & 2395 \\
3 & 2335 \\
3 & 2273\end{array}$ & $\begin{array}{l}59 \\
60 \\
60 \\
61 \\
62\end{array}$ \\
\hline $\begin{array}{l}74.5 \\
74.6 \\
74.7 \\
74.8 \\
74.9 \\
75.0\end{array}$ & $\begin{array}{ll}0.4373 & 5815 \\
0.4383 & 1198 \\
0.4392 & 7063 \\
0.4402 & 3414 \\
0.4412 & 0258 \\
0.4421 & 7599\end{array}$ & $\begin{array}{ll}9 & 5583 \\
9 & 5865 \\
9 & 6352 \\
9 & 6844 \\
9 & 7341 \\
9 & 7844\end{array}$ & $\begin{array}{l}482 \\
487 \\
492 \\
498 \\
503 \\
509\end{array}$ & 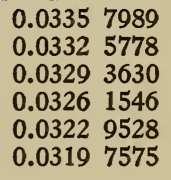 & $\begin{array}{ll}3 & 2211 \\
3 & 2148 \\
3 & 2084 \\
3 & 2019 \\
3 & 1952 \\
3 & 1885\end{array}$ & $\begin{array}{l}63 \\
64 \\
65 \\
66 \\
67 \\
68\end{array}$ \\
\hline
\end{tabular}


TABLE XIII-Continued

\begin{tabular}{|c|c|c|c|c|c|c|}
\hline$\gamma$ & $\log F$ & $\triangle_{1}$ & $\triangle_{2}$ & $\log E$ & $\Delta_{1}$ & $\Delta_{2}$ \\
\hline $\begin{array}{l}75.0 \\
75.1 \\
75.2 \\
75.3 \\
75.4\end{array}$ & $\begin{array}{ll}0.4421 & 7599 \\
0.4431 & 5444 \\
0.4441 & 3797 \\
0.4451 & 2664 \\
0.4461 & 2051\end{array}$ & $\begin{array}{ll}9 & 7844 \\
9 & 8353 \\
9 & 8867 \\
9 & 9387 \\
9 & 9913\end{array}$ & $\begin{array}{l}509 \\
514 \\
520 \\
526 \\
532\end{array}$ & 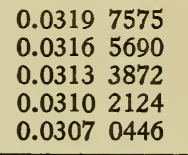 & $\begin{array}{ll}3 & 1885 \\
3 & 1817 \\
3 & 1748 \\
3 & 1678 \\
3 & 1607\end{array}$ & $\begin{array}{l}68 \\
69 \\
70 \\
71 \\
72\end{array}$ \\
\hline $\begin{array}{l}75.5 \\
75.6 \\
75.7 \\
75.8 \\
75.9\end{array}$ & $\begin{array}{ll}0.4471 & 1965 \\
0.4481 & 2410 \\
0.4491 & 3394 \\
0.4501 & 4922 \\
0.4511 & 7001\end{array}$ & $\begin{array}{ll}10 & 0446 \\
10 & 0984 \\
10 & 1528 \\
10 & 2079 \\
10 & 2637\end{array}$ & $\begin{array}{l}538 \\
544 \\
551 \\
557 \\
564\end{array}$ & 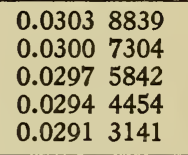 & $\begin{array}{ll}3 & 1535 \\
3 & 1462 \\
3 & 1388 \\
3 & .1313 \\
3 & 1237\end{array}$ & $\begin{array}{l}73 \\
74 \\
75 \\
76 \\
77\end{array}$ \\
\hline $\begin{array}{l}76.0 \\
76.1 \\
76.2 \\
76.3 \\
76.4\end{array}$ & $\begin{array}{ll}0.4521 & 9638 \\
0.4532 & 2839 \\
0.4542 & 6610 \\
0.4553 & 0959 \\
0.4563 & 5893\end{array}$ & $\begin{array}{ll}10 & 3201 \\
10 & 3771 \\
10 & 4349 \\
10 & 4934 \\
10 & 5526\end{array}$ & $\begin{array}{l}571 \\
578 \\
585 \\
592 \\
599\end{array}$ & $\begin{array}{ll}0.0288 & 1904 \\
0.0285 & 0745 \\
0.0281 & 9664 \\
0.0278 & 8663 \\
0.0275 & 7742\end{array}$ & $\begin{array}{ll}3 & 1159 \\
3 & 1081 \\
3 & 1002 \\
3 & 0921 \\
3 & 0839\end{array}$ & $\begin{array}{l}78 \\
79 \\
80 \\
82 \\
83\end{array}$ \\
\hline $\begin{array}{l}76.5 \\
76.6 \\
76.7 \\
76.8 \\
76.9\end{array}$ & $\begin{array}{ll}0.4574 & 1419 \\
0.4584 & 7545 \\
0.4595 & 4278 \\
0.4606 & 1625 \\
0.4616 & 9594 \\
\end{array}$ & $\begin{array}{ll}10 & 6126 \\
10 & 6733 \\
10 & 7347 \\
10 & 7970 \\
10 & 8600 \\
\end{array}$ & $\begin{array}{l}607 \\
615 \\
622 \\
630 \\
639 \\
\end{array}$ & $\begin{array}{l}0.02726902 \\
0.02696145 \\
0.02665472 \\
0.0263 \quad 4884 \\
0.02604382 \\
\end{array}$ & $\begin{array}{ll}3 & 0757 \\
3 & 0673 \\
3 & 0588 \\
3 & 0502 \\
3 & 0415 \\
\end{array}$ & $\begin{array}{l}84 \\
85 \\
86 \\
87 \\
88\end{array}$ \\
\hline $\begin{array}{l}77.0 \\
77.1 \\
77.2 \\
77.3 \\
77.4\end{array}$ & $\begin{array}{l}0.4627 \quad 8195 \\
0.46387433 \\
0.46497319 \\
0.46607860 \\
0.46719066\end{array}$ & $\begin{array}{ll}10 & 9239 \\
10 & 9886 \\
11 & 0541 \\
11 & 1206 \\
11 & 1879\end{array}$ & $\begin{array}{l}647 \\
656 \\
664 \\
673 \\
682 \\
\end{array}$ & $\begin{array}{lll}0.0257 & 3967 \\
0.0254 & 3640 \\
0.0251 & 3403 \\
0.0248 & 3257 \\
0.0245 & 3202\end{array}$ & $\begin{array}{ll}3 & 0327 \\
3 & 0237 \\
3 & 0147 \\
3 & 0055 \\
2 & 9962\end{array}$ & $\begin{array}{l}89 \\
91 \\
92 \\
93 \\
94\end{array}$ \\
\hline $\begin{array}{l}77.5 \\
77.6 \\
77.7 \\
77.8 \\
77.9\end{array}$ & 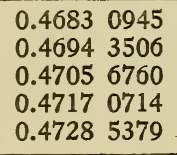 & $\begin{array}{ll}11 & 2561 \\
11 & 3253 \\
11 & 3954 \\
11 & 4665 \\
11 & 5386\end{array}$ & $\begin{array}{l}692 \\
701 \\
711 \\
721 \\
731 \\
\end{array}$ & 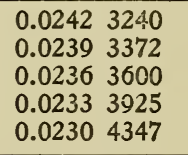 & $\begin{array}{ll}2 & 9868 \\
2 & 9772 \\
2 & 9676 \\
2 & 9578 \\
2 & 9479\end{array}$ & $\begin{array}{r}95 \\
97 \\
98 \\
99 \\
100\end{array}$ \\
\hline $\begin{array}{l}78.0 \\
78.1 \\
78.2 \\
78.3 \\
78.4\end{array}$ & 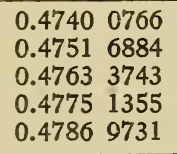 & $\begin{array}{ll}11 & 6118 \\
11 & 6860 \\
11 & 7612 \\
11 & 8376 \\
11 & 9150\end{array}$ & $\begin{array}{l}742 \\
753 \\
764 \\
775 \\
786\end{array}$ & 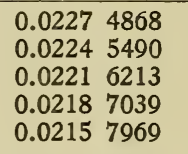 & $\begin{array}{ll}2 & 9378 \\
2 & 9277 \\
2 & 9174 \\
2 & 9070 \\
2 & 8964\end{array}$ & $\begin{array}{l}102 \\
103 \\
104 \\
105 \\
107\end{array}$ \\
\hline \begin{tabular}{|l|}
78.5 \\
78.6 \\
78.7 \\
78.8 \\
78.9
\end{tabular} & $\begin{array}{ll}0.4798 & 8881 \\
0.4810 & 8818 \\
0.4822 & 9553 \\
0.4835 & 1098 \\
0.4847 & 3466\end{array}$ & $\begin{array}{ll}11 & 9937 \\
12 & 0735 \\
12 & 1545 \\
12 & 2368 \\
12 & 3203\end{array}$ & $\begin{array}{l}798 \\
810 \\
823 \\
835 \\
848\end{array}$ & $\begin{array}{ll}0.0212 & 9005 \\
0.0210 & 0148 \\
0.0207 & 1398 \\
0.0204 & 2758 \\
0.0201 & 4229\end{array}$ & $\begin{array}{ll}2 & 8858 \\
2 & 8750 \\
2 & 8640 \\
2 & 8529 \\
2 & 8417\end{array}$ & $\begin{array}{l}108 \\
109 \\
111 \\
112 \\
113\end{array}$ \\
\hline $\begin{array}{l}79.0 \\
79.1 \\
79.2 \\
79.3 \\
79.4\end{array}$ & $\begin{array}{ll}0.4859 & 6669 \\
0.4872 & 0721 \\
0.4884 & 5635 \\
0.4897 & 1424 \\
0.4909 & 8103\end{array}$ & $\begin{array}{ll}12 & 4052 \\
12 & 4914 \\
12 & 5789 \\
12 & 6679 \\
12 & 7583\end{array}$ & $\begin{array}{l}862 \\
876 \\
890 \\
904 \\
919\end{array}$ & $\begin{array}{ll}0.0198 & 5811 \\
0.0195 & 7507 \\
0.0192 & 9318 \\
0.0190 & 1246 \\
0.0187 & 3291\end{array}$ & $\begin{array}{ll}2 & 8304 \\
2 & 8189 \\
2 & 8073 \\
2 & 7955 \\
2 & 7836\end{array}$ & $\begin{array}{l}115 \\
116 \\
118 \\
119 \\
120\end{array}$ \\
\hline $\begin{array}{l}79.5 \\
79.6 \\
79.7 \\
79.8 \\
79.9 \\
80.0\end{array}$ & $\begin{array}{ll}0.4922 & 5687 \\
0.4935 & 4189 \\
0.4948 & 3626 \\
0.4961 & 4013 \\
0.4974 & 5367 \\
0.4987 & 7703\end{array}$ & $\begin{array}{ll}12 & 8503 \\
12 & 9437 \\
13 & 0387 \\
13 & 1353 \\
13 & 2336 \\
13 & 3336\end{array}$ & $\begin{array}{r}934 \\
950 \\
966 \\
983 \\
1000 \\
1018\end{array}$ & $\begin{array}{ll}0.0184 & 5454 \\
0.0181 & 7739 \\
0.0179 & 0145 \\
0.0176 & 2675 \\
0.0173 & 5330 \\
0.0170 & 8111\end{array}$ & $\begin{array}{ll}2 & 7716 \\
2 & 7594 \\
2 & 7470 \\
2 & 7345 \\
2 & 7219 \\
2 & 7091\end{array}$ & $\begin{array}{l}122 \\
123 \\
125 \\
126 \\
128 \\
129\end{array}$ \\
\hline
\end{tabular}


TABLE XIII--Continued

\begin{tabular}{|c|c|c|c|c|c|c|}
\hline$\gamma$ & $\log F$ & $\triangle_{1}$ & $\Delta_{2}$ & $\log E$ & $\Delta_{1}$ & $\triangle_{2}$ \\
\hline $\begin{array}{l}80.0 \\
80.1 \\
80.2 \\
80.3 \\
80.4 \\
\end{array}$ & 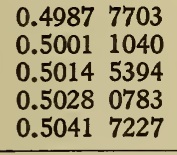 & $\begin{array}{ll}13 & 3336 \\
13 & 4354 \\
13 & 5390 \\
13 & 6444 \\
13 & 7517 \\
\end{array}$ & $\begin{array}{l}1018 \\
1036 \\
1054 \\
1073 \\
1093 \\
\end{array}$ & 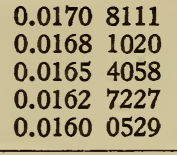 & $\begin{array}{ll}2 & 7091 \\
2 & 6962 \\
2 & 6831 \\
2 & 6698 \\
2 & 6564 \\
\end{array}$ & $\begin{array}{l}129 \\
131 \\
132 \\
134 \\
136 \\
\end{array}$ \\
\hline $\begin{array}{l}80.5 \\
80.6 \\
80.7 \\
80.8 \\
80.9 \\
\end{array}$ & 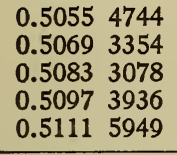 & $\begin{array}{ll}13 & 8610 \\
13 & 9724 \\
14 & 0858 \\
14 & 2014 \\
14 & 3192 \\
\end{array}$ & $\begin{array}{l}1113 \\
1134 \\
1156 \\
1178 \\
1201 \\
\end{array}$ & 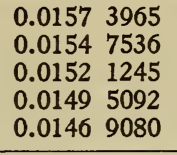 & $\begin{array}{ll}2 & 6429 \\
2 & 6291 \\
2 & 6153 \\
2 & 6012 \\
2 & 5870 \\
\end{array}$ & $\begin{array}{l}137 \\
139 \\
140 \\
142 \\
144 \\
\end{array}$ \\
\hline $\begin{array}{l}81.0 \\
81.1 \\
81.2 \\
81.3 \\
81.4\end{array}$ & 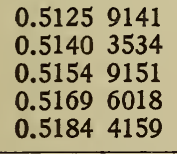 & $\begin{array}{ll}14 & 4393 \\
14 & 5617 \\
14 & 6867 \\
14 & 8141 \\
14 & 9441 \\
\end{array}$ & $\begin{array}{l}1225 \\
1249 \\
1274 \\
1300 \\
1327 \\
\end{array}$ & 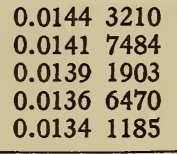 & $\begin{array}{ll}2 & 5726 \\
2 & 5581 \\
2 & 5433 \\
2 & 5285 \\
2 & 5134 \\
\end{array}$ & $\begin{array}{l}145 \\
147 \\
149 \\
151 \\
152\end{array}$ \\
\hline $\begin{array}{l}81.5 \\
81.6 \\
81.7 \\
81.8 \\
81.9\end{array}$ & $\begin{array}{ll}0.5199 & 3600 \\
0.5214 & 4369 \\
0.5229 & 6493 \\
0.5245 & 0001 \\
0.5260 & 4923 \\
\end{array}$ & $\begin{array}{ll}15 & 0769 \\
15 & 2124 \\
15 & 3508 \\
15 & 4922 \\
15 & 6366 \\
\end{array}$ & $\begin{array}{l}1355 \\
1384 \\
1414 \\
1445 \\
1477 \\
\end{array}$ & $\begin{array}{ll}0.0131 & 6052 \\
0.0129 & 1070 \\
0.0126 & 6243 \\
0.0124 & 1572 \\
0.0121 & 7058\end{array}$ & $\begin{array}{ll}2 & 4981 \\
2 & 4827 \\
2 & 4671 \\
2 & 4513 \\
2 & 4354\end{array}$ & $\begin{array}{l}154 \\
156 \\
158 \\
160 \\
162\end{array}$ \\
\hline $\begin{array}{l}82.0 \\
82.1 \\
82.2 \\
82.3 \\
82.4\end{array}$ & $\begin{array}{ll}0.5276 & 1289 \\
0.5291 & 9132 \\
0.5307 & 8485 \\
0.5323 & 9381 \\
0.5340 & 1857\end{array}$ & $\begin{array}{ll}15 & 7843 \\
15 & 9352 \\
16 & 0896 \\
16 & 2476 \\
16 & 4092 \\
\end{array}$ & $\begin{array}{l}1510 \\
1544 \\
1579 \\
1616 \\
1655\end{array}$ & 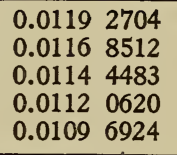 & $\begin{array}{ll}2 & 4192 \\
2 & 4029 \\
2 & 3863 \\
2 & 3696 \\
2 & 3527\end{array}$ & $\begin{array}{l}163 \\
165 \\
167 \\
169 \\
171\end{array}$ \\
\hline $\begin{array}{l}82.5 \\
82.6 \\
82.7 \\
82.8 \\
82.9\end{array}$ & $\begin{array}{ll}0.5356 & 5949 \\
0.5373 & 1696 \\
0.5389 & 9137 \\
0.5406 & 8313 \\
0.5423 & 9268\end{array}$ & $\begin{array}{ll}16 & 5747 \\
16 & 7441 \\
16 & 9177 \\
17 & 0955 \\
17 & 2778\end{array}$ & $\begin{array}{l}1694 \\
1736 \\
1779 \\
1823 \\
1870\end{array}$ & $\begin{array}{ll}0.0107 & 3397 \\
0.0105 & 0041 \\
0.0102 & 6859 \\
0.0100 & 3851 \\
0.0098 & 1021\end{array}$ & $\begin{array}{ll}2 & 3356 \\
2 & 3183 \\
2 & 3007 \\
2 & 2830 \\
2 & 2651\end{array}$ & $\begin{array}{l}173 \\
175 \\
177 \\
179 \\
181\end{array}$ \\
\hline $\begin{array}{l}83.0 \\
83.1 \\
83.2 \\
83.3 \\
83.4\end{array}$ & $\begin{array}{ll}0.5441 & 2047 \\
0.5458 & 6695 \\
0.5476 & 3260 \\
0.5494 & 1795 \\
0.5512 & 2350\end{array}$ & $\begin{array}{ll}17 & 4648 \\
17 & 6566 \\
17 & 8534 \\
18 & 0555 \\
18 & 2631\end{array}$ & $\begin{array}{l}1918 \\
1968 \\
2021 \\
2076 \\
2133\end{array}$ & $\begin{array}{ll}0.0095 & 8371 \\
0.0093 & 5902 \\
0.0091 & 3616 \\
0.0089 & 1517 \\
0.0086 & 9605\end{array}$ & $\begin{array}{ll}2 & 2469 \\
2 & 2285 \\
2 & 2100 \\
2 & 1912 \\
2 & 1721\end{array}$ & $\begin{array}{l}184 \\
186 \\
188 \\
190 \\
193\end{array}$ \\
\hline \begin{tabular}{|l|}
83.5 \\
83.6 \\
83.7 \\
83.8 \\
83.9
\end{tabular} & 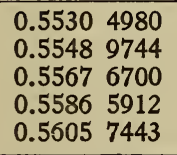 & $\begin{array}{ll}18 & 4764 \\
18 & 6956 \\
18 & 9211 \\
19 & 1532 \\
19 & 3921\end{array}$ & $\begin{array}{l}2193 \\
2255 \\
2320 \\
2389 \\
2460\end{array}$ & $\begin{array}{ll}0.0084 & 7884 \\
0.0082 & 6355 \\
0.0080 & 5021 \\
0.0078 & 3884 \\
0.0076 & 2947\end{array}$ & $\begin{array}{ll}2 & 1529 \\
2 & 1334 \\
2 & 1137 \\
2 & 0937 \\
2 & 0735\end{array}$ & $\begin{array}{l}195 \\
197 \\
199 \\
202 \\
204\end{array}$ \\
\hline $\begin{array}{l}84.0 \\
84.1 \\
84.2 \\
84.3 \\
84.4\end{array}$ & $\begin{array}{ll}0.5625 & 1364 \\
0.5644 & 7745 \\
0.5664 & 6661 \\
0.5684 & 8192 \\
0.5705 & 2420\end{array}$ & $\begin{array}{ll}19 & 6381 \\
19 & 8916 \\
20 & 1531 \\
20 & 4228 \\
20 & 7012\end{array}$ & $\begin{array}{l}2535 \\
2614 \\
2697 \\
2784 \\
2875\end{array}$ & $\begin{array}{ll}0.0074 & 2211 \\
0.0072 & 1680 \\
0.0070 & 1356 \\
0.0068 & 1241 \\
0.0066 & 1338\end{array}$ & $\begin{array}{ll}2 & 0531 \\
2 & 0324 \\
2 & 0115 \\
1 & 9903 \\
1 & 9689\end{array}$ & $\begin{array}{l}207 \\
209 \\
212 \\
214 \\
217\end{array}$ \\
\hline $\begin{array}{l}84.5 \\
84.6 \\
84.7 \\
84.8 \\
84.9 \\
85.0\end{array}$ & $\begin{array}{ll}0.5725 & 9431 \\
0.5746 & 9318 \\
0.5768 & 2177 \\
0.5789 & 8109 \\
0.5811 & 7221 \\
0.5833 & 9626\end{array}$ & $\begin{array}{ll}20 & 9887 \\
21 & 2859 \\
21 & 5932 \\
21 & 9112 \\
22 & 2405 \\
22 & 5818\end{array}$ & $\begin{array}{l}2972 \\
3073 \\
3180 \\
3293 \\
3413 \\
3539\end{array}$ & $\begin{array}{ll}0.0064 & 1649 \\
0.0062 & 2177 \\
0.0060 & 2925 \\
0.0058 & 3896 \\
0.0056 & 5092 \\
0.0054 & 6516\end{array}$ & $\begin{array}{ll}1 & 9472 \\
1 & 9252 \\
1 & 9029 \\
1 & 8804 \\
1 & 8576 \\
1 & 8345\end{array}$ & $\begin{array}{l}220 \\
222 \\
225 \\
228 \\
231 \\
234\end{array}$ \\
\hline
\end{tabular}


TABLE XIII-Continued

\begin{tabular}{|c|c|c|c|c|c|c|}
\hline$\gamma$ & $\log F$ & $\Delta_{1}$ & $\triangle_{2}$ & $\log E$ & $\Delta_{1}$ & $\Delta_{2}$ \\
\hline $\begin{array}{l}85.0 \\
85.1 \\
85.2 \\
85.3 \\
85.4\end{array}$ & 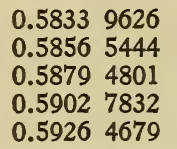 & $\begin{array}{ll}22 & 5818 \\
22 & 9357 \\
23 & 3031 \\
23 & 6846 \\
24 & 0813\end{array}$ & $\begin{array}{l}3539 \\
3673 \\
3816 \\
3967 \\
4127\end{array}$ & 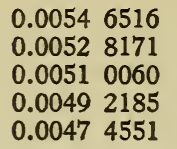 & $\begin{array}{ll}1 & 8345 \\
1 & 8111 \\
1 & 7874 \\
1 & 7634 \\
1 & 7391\end{array}$ & $\begin{array}{l}234 \\
237 \\
240 \\
243 \\
246\end{array}$ \\
\hline $\begin{array}{l}85.5 \\
85.6 \\
85.7 \\
85.8 \\
85.9\end{array}$ & $\begin{array}{ll}0.5950 & 5492 \\
0.5975 & 0432 \\
0.5999 & 9671 \\
0.6025 & 3391 \\
0.6051 & 1788\end{array}$ & $\begin{array}{ll}24 & 4940 \\
24 & 9239 \\
25 & 3720 \\
25 & 8396 \\
26 & 3281\end{array}$ & $\begin{array}{l}4299 \\
4481 \\
4676 \\
4885 \\
5109\end{array}$ & 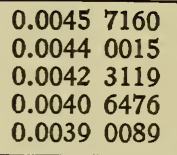 & $\begin{array}{ll}1 & 7145 \\
1 & 6896 \\
1 & 6643 \\
1 & 6387 \\
1 & 6127\end{array}$ & $\begin{array}{l}249 \\
253 \\
256 \\
260 \\
263\end{array}$ \\
\hline $\begin{array}{l}86.0 \\
86.1 \\
86.2 \\
86.3 \\
86.4\end{array}$ & 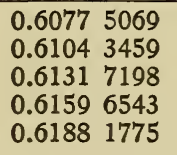 & $\begin{array}{ll}26 & 8390 \\
27 & 3739 \\
27 & 9346 \\
28 & 5231 \\
29 & 1418\end{array}$ & $\begin{array}{l}5349 \\
5607 \\
5886 \\
6186 \\
6512\end{array}$ & 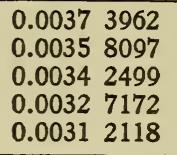 & $\begin{array}{ll}1 & 5864 \\
1 & 5598 \\
1 & 5327 \\
1 & 5053 \\
1 & 4775\end{array}$ & $\begin{array}{l}267 \\
270 \\
274 \\
278 \\
282\end{array}$ \\
\hline $\begin{array}{l}86.5 \\
86.6 \\
86.7 \\
86.8 \\
86.9\end{array}$ & $\begin{array}{ll}0.6217 & 3193 \\
0.6247 & 1122 \\
0.6277 & 5916 \\
0.6308 & 7958 \\
0.6340 & 7668\end{array}$ & $\begin{array}{ll}29 & 7929 \\
30 & 4794 \\
31 & 2042 \\
31 & 9709 \\
32 & 7834\end{array}$ & $\begin{array}{l}6865 \\
7248 \\
7667 \\
8124 \\
8626\end{array}$ & $\begin{array}{ll}0.0029 & 7343 \\
0.0028 & 2850 \\
0.0026 & 8642 \\
0.0025 & 4725 \\
0.0024 & 1103\end{array}$ & $\begin{array}{ll}1 & 4493 \\
1 & 4207 \\
1 & 3917 \\
1 & 3622 \\
1 & 3323\end{array}$ & $\begin{array}{l}286 \\
290 \\
295 \\
299 \\
304\end{array}$ \\
\hline $\begin{array}{l}87.0 \\
87.1 \\
87.2 \\
87.3 \\
87.4\end{array}$ & $\begin{array}{ll}0.6373 & 5501 \\
0.6407 & 1961 \\
0.6441 & 7597 \\
0.6477 & 3019 \\
0.6513 & 8900\end{array}$ & $\begin{array}{ll}33 & 6459 \\
34 & 5636 \\
35 & 5422 \\
36 & 5881 \\
37 & 7089\end{array}$ & $\begin{array}{r}9177 \\
9785 \\
10459 \\
11208 \\
12043\end{array}$ & 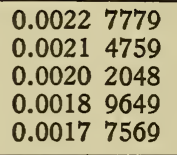 & $\begin{array}{ll}1 & 3020 \\
1 & 2712 \\
1 & 2398 \\
1 & 2080 \\
1 & 1757\end{array}$ & $\begin{array}{l}308 \\
313 \\
318 \\
324 \\
329\end{array}$ \\
\hline $\begin{array}{l}87.5 \\
87.6 \\
87.7 \\
87.8 \\
87.9\end{array}$ & $\begin{array}{ll}0.6551 & 5989 \\
0.6590 & 5121 \\
0.6630 & 7233 \\
0.6672 & 3380 \\
0.6715 & 4757\end{array}$ & $\begin{array}{ll}38 & 9132 \\
40 & 2112 \\
41 & 6147 \\
43 & 1377 \\
44 & 7967\end{array}$ & $\begin{array}{l}12980 \\
14035 \\
15230 \\
16590 \\
18149\end{array}$ & $\begin{array}{ll}0.0016 & 5813 \\
0.0015 & 4385 \\
0.0014 & 3292 \\
0.0013 & 2540 \\
0.0012 & 2134\end{array}$ & $\begin{array}{ll}1 & 1428 \\
1 & 1093 \\
1 & 0753 \\
1 & 0406 \\
1 & 0053 \\
\end{array}$ & $\begin{array}{l}335 \\
340 \\
347 \\
353 \\
360\end{array}$ \\
\hline $\begin{array}{l}88.0 \\
88.1 \\
88.2 \\
88.3 \\
88.4\end{array}$ & 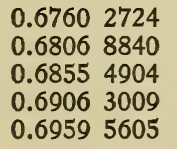 & $\begin{array}{ll}46 & 6116 \\
48 & 6064 \\
50 & 8104 \\
53 & 2597 \\
55 & 9993\end{array}$ & $\begin{array}{l}19948 \\
22040 \\
24492 \\
27396 \\
30870\end{array}$ & 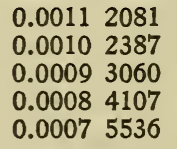 & $\begin{array}{l}9693 \\
9327 \\
8953 \\
8571 \\
8181\end{array}$ & $\begin{array}{l}367 \\
374 \\
382 \\
390 \\
399\end{array}$ \\
\hline $\begin{array}{l}88.5 \\
88.6 \\
88.7 \\
88.8 \\
88.9 \\
\end{array}$ & 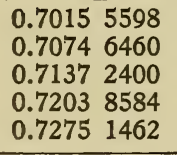 & $\begin{array}{ll}59 & 0862 \\
62 & 5940 \\
66 & 6184 \\
71 & 2878 \\
76 & 7773 \\
\end{array}$ & $\begin{array}{l}35077 \\
40245 \\
46693 \\
54895 \\
65561 \\
\end{array}$ & $\begin{array}{l}0.00067355 \\
0.00059573 \\
0.0005 \quad 2199 \\
0.00045242 \\
0.00038715 \\
\end{array}$ & $\begin{array}{l}7782 \\
7374 \\
6956 \\
6527 \\
6087 \\
\end{array}$ & $\begin{array}{l}408 \\
418 \\
429 \\
441 \\
453 \\
\end{array}$ \\
\hline $\begin{array}{l}89.0 \\
89.1 \\
89.2 \\
89.3 \\
89.4\end{array}$ & $\begin{array}{ll}0.7351 & 9234 \\
0.7435 & 2568 \\
0.7526 & 5714 \\
0.7627 & 8356 \\
0.7741 & 8844\end{array}$ & $\begin{array}{rr}83 & 3334 \\
91 & 3146 \\
101 & 2642 \\
114 & 0489 \\
131 & 1464 \\
\end{array}$ & $\begin{array}{r}79812 \\
99496 \\
127847 \\
170975 \\
241655 \\
\end{array}$ & $\begin{array}{ll}0.0003 & 2628 \\
0.0002 & 6995 \\
0.0002 & 1829 \\
0.0001 & 7146 \\
0.0001 & 2965 \\
\end{array}$ & $\begin{array}{l}5633 \\
5166 \\
4683 \\
4181 \\
3660 \\
\end{array}$ & $\begin{array}{l}467 \\
483 \\
501 \\
522 \\
546 \\
\end{array}$ \\
\hline $\begin{array}{l}89.5 \\
89.6 \\
89.7 \\
89.8 \\
89.9 \\
90.0\end{array}$ & $\begin{array}{cc}0.7873 & 0308 \\
0.8028 & 3427 \\
0.8220 & 7240 \\
0.8478 & 1809 \\
0.8885 & 7889 \\
\text { Inf. }\end{array}$ & $\begin{array}{ll}155 & 3119 \\
192 & 3813 \\
257 & 4569 \\
407 & 6079\end{array}$ & $\begin{array}{r}370693 \\
650756 \\
1501510\end{array}$ & 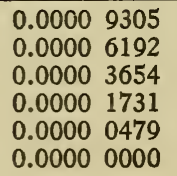 & $\begin{array}{r}3114 \\
2538 \\
1923 \\
1253 \\
479\end{array}$ & $\begin{array}{l}576 \\
615 \\
670 \\
774\end{array}$ \\
\hline
\end{tabular}


The preceding table of logarithms of the elliptic integrals of the first and second kinds is taken from Legendre's Traité des Fonctions Elliptiques, volume 2, Table I. The values from $45^{\circ}$ to $90^{\circ}$ are given for intervals of $\mathrm{O}^{\circ} \mathrm{r}$. The values from $0^{\circ}$ to $45^{\circ}$, which are comparatively seldom required, have been omitted. For formula and table to be used in interpolation, see page 2 I 4 .

\section{TABLE XIV}

Binominal Coefficients for Interpolation by Differences

\begin{tabular}{|c|c|c|c|c|c|c|c|c|c|c|c|}
\hline \multirow{2}{*}{$\mathbf{k}$} & \multicolumn{2}{|c|}{$\begin{array}{c}\text { Coefficients of } \\
\Delta_{2} \text { and } \Delta_{3}\end{array}$} & \multirow{2}{*}{ k } & \multicolumn{2}{|c|}{$\begin{array}{c}\text { Coefficients of } \\
\Delta_{2} \text { and } \Delta_{3}\end{array}$} & \multirow{2}{*}{$\mathbf{k}$} & \multicolumn{2}{|c|}{$\begin{array}{c}\text { Coefficients of } \\
\Delta_{2} \text { and } \Delta_{3}\end{array}$} & \multirow{2}{*}{$\mathrm{k}$} & \multicolumn{2}{|c|}{$\begin{array}{l}\text { Coefficients of } \\
\Delta_{2} \text { and } \Delta_{3}\end{array}$} \\
\hline & $\mathbf{K}_{2}$ & $\mathbf{K}_{3}$ & & $\mathrm{~K}_{2}$ & $\mathrm{~K}_{3}$ & & $\mathbf{K}_{2}$ & $\mathrm{~K}_{3}$ & & $\mathrm{~K}_{2}$ & $\mathbf{K}_{3}$ \\
\hline 0.01 & 0.005 & +0.003 & 0.26 & 0.096 & +0.056 & 0.51 & -0.125 & +0.062 & 0.76 & -0.091 & +0.038 \\
\hline .02 & -.010 & +.006 & .27 & - .099 & +.057 & .52 & -.125 & +.062 & .77 & -.089 & +.036 \\
\hline .03 & -.015 & +.010 & .28 & - .101 & +.058 & .53 & -.125 & +.061 & .78 & -.086 & +.035 \\
\hline .04 & -.019 & +.013 & .29 & -.103 & +.059 & .54 & -.124 & +.060 & .79 & -.083 & +.033 \\
\hline .05 & -.024 & +.015 & .30 & -.105 & +.060 & .55 & -.124 & +.060 & .80 & -.080 & +.032 \\
\hline .06 & -.028 & +.018 & .31 & -.107 & +.060 & .56 & -.124 & +.059 & .81 & -.077 & +.031 \\
\hline .07 & -.033 & +.021 & .32 & -.109 & +.061 & .57 & -.123 & +.058 & .82 & -.074 & +.029 \\
\hline .08 & -.037 & +.024 & .33 & -.111 & +.062 & .58 & -.122 & +.058 & .83 & -.071 & +.028 \\
\hline .09 & -.041 & +.026 & .34 & -.112 & +.062 & .59 & -.121 & +.057 & .84 & -.067 & +.026 \\
\hline .10 & -.045 & +.028 & .35 & -.114 & +.063 & .60 & -.120 & +.056 & .85 & -.064 & +.024 \\
\hline .11 & -.049 & +.031 & .36 & -.115 & +.0 & .61 & -.119 & +.055 & .86 & -.060 & +.023 \\
\hline .12 & -.053 & +.0 & .37 & - .117 & +.0 & .62 & -.118 & +.054 & .87 & -.057 & +.021 \\
\hline .13 & -.057 & +.035 & .38 & -.118 & +.064 & .63 & -.117 & +.053 & .88 & -.053 & +.020 \\
\hline .14 & -.060 & +.037 & .39 & -.119 & +.064 & .64 & -.115 & +.052 & .89 & -.049 & +.018 \\
\hline .15 & -.064 & +.039 & .40 & -.120 & +.064 & .65 & -.114 & +.051 & .90 & -.045 & +.016 \\
\hline .16 & -.067 & +.041 & .41 & -.121 & +.064 & .66 & -.112 & +.050 & .91 & -.041 & +.015 \\
\hline .17 & -.071 & +.043 & .42 & -.122 & +.064 & .67 & -.111 & +.049 & .92 & -.037 & +.013 \\
\hline .18 & -.074 & +.0 & .43 & -.123 & +.0 & .68 & -.109 & + & .93 & -.033 & +.012 \\
\hline .19 & -.077 & +.046 & .44 & -.123 & +.064 & .69 & -.107 & +.047 & .94 & -.028 & +.010 \\
\hline .20 & -.080 & +.048 & .45 & -.124 & +.064 & .70 & -.105 & +.045 & .95 & -.024 & +.008 \\
\hline .21 & -.083 & +.049 & .46 & -.124 & +.064 & .71 & -. .103 & +.044 & .96 & -.019 & +.007 \\
\hline .22 & -.086 & +.051 & .47 & -.125 & +.064 & .72 & -.101 & +.043 & .97 & -.015 & +.005 \\
\hline .23 & -.089 & +.052 & .48 & -.125 & +.063 & .73 & -.099 & +.042 & .98 & -.010 & +.003 \\
\hline .24 & -.091 & +.053 & .49 & .125 & +.06 & .74 & -.096 & + & .99 & -.005 & \\
\hline .25 & -.094 & +.055 & .50 & -.125 & +.063 & .75 & -.094 & +.039 & 1.00 & -.000 & +.000 \\
\hline
\end{tabular}




\section{INTERPOLATION FORMULA}

$$
\begin{aligned}
f(a+h)=f(a)+k \triangle_{1}+\frac{k(k-\mathrm{I})}{2 !} \triangle_{2}+\frac{k(k-\mathrm{I})(k-2)}{3 !} \triangle_{3} \\
\quad+\frac{k(k-\mathrm{I})(k-2)(k-3)}{4 !} \triangle_{4}+\cdots
\end{aligned}
$$

or, $f(a+h)=f(a)+k \triangle_{1}+K_{2} \triangle_{2}+K_{3} \triangle_{3}+\cdots$

where the constants $K_{2}$ and $K_{3}$ are given in the above table as functions of $k$ and

$$
k=\frac{h}{\delta}
$$

where $h$ is the remainder above the value of $a$ for which the function is given in the table, and $\delta$ is the increment of $\alpha$ in the table.

\section{ILLUSTRATION}

To find the value of $\log F$ for $49^{\circ}$ I $5^{\prime} 36^{\prime \prime}=49^{\circ} \cdot 260$

For $49: 2 \quad \log F=0.2836$ 31 $30=f(a)$

$$
h=.06, \quad \delta=0.1 \quad k=0.6
$$

From Table XIV,

$$
\begin{aligned}
& K_{2}=-.120 \\
& K_{3}=+.056
\end{aligned}
$$

From Table XIII,

$$
\begin{array}{lr}
\triangle_{1}= & 39338 \\
\triangle_{2}= & \text { I I } 7 \\
\triangle_{3}= & \text { I }
\end{array}
$$

Substituting these values of $K_{2}, K_{3}, \triangle_{1}, \triangle_{2}, \triangle_{3}$ in formula (b) above we have as the value of $\log F$ for the given angle $\log F=0.2836$ 3 I $30+0.00023603-0.00000014=0.283867$ I 9 . 


\section{TABLE XV}

Values of the Quantities $q-\frac{l}{2}$ or $q_{1}-\frac{l_{1}}{2}$ and $\log _{10}(1+\epsilon)$ with Argument $q$ or $q_{1}$

$$
\begin{aligned}
q-\frac{l}{2} & =2\left(\frac{l}{2}\right)^{5}+15\left(\frac{l}{2}\right)^{9}+\cdots \\
\epsilon & =3 q^{4}-4 q^{6}+9 q^{8}-12 q^{10}+\cdots \\
q_{1}-\frac{l_{1}}{2} & =2\left(\frac{l_{1}}{2}\right)^{5}+\mathrm{r} 5\left(\frac{l_{1}}{2}\right)^{9}+\cdots
\end{aligned}
$$

\begin{tabular}{|c|c|c|c|c|c|c|}
\hline $\begin{array}{c}q \\
\text { or } q_{1}\end{array}$ & $\begin{array}{l}q-\frac{1}{2} \\
\text { or } q_{1}-\frac{1_{1}}{2}\end{array}$ & $\triangle$ & $\varepsilon$ & $\Delta$ & $\log _{10}(1+\varepsilon)$ & $\triangle$ \\
\hline $\begin{array}{l}0.020 \\
.022 \\
.024 \\
.026 \\
.028\end{array}$ & $\begin{array}{r}0.00000001 \\
.00000001 \\
.00000002 \\
.00000002 \\
.00000003\end{array}$ & $\begin{array}{l}0 \\
1 \\
0 \\
1 \\
2\end{array}$ & $\begin{array}{r}0.00000048 \\
.00000070 \\
.00000099 \\
.00000137 \\
.00000184\end{array}$ & $\begin{array}{l}22 \\
29 \\
38 \\
47 \\
59\end{array}$ & $\begin{array}{r}0.00000021 \\
.00000030 \\
.00000043 \\
.00000059 \\
.00000080\end{array}$ & $\begin{array}{r}9 \\
13 \\
16 \\
21 \\
25\end{array}$ \\
\hline $\begin{array}{r}0.030 \\
.032 \\
.034 \\
.036 \\
.038\end{array}$ & $\begin{array}{r}0.00000005 \\
.00000007 \\
.00000009 \\
.00000012 \\
.00000016\end{array}$ & $\begin{array}{l}2 \\
2 \\
3 \\
4 \\
5\end{array}$ & $\begin{array}{r}0.00000243 \\
.00000314 \\
.00000400 \\
.00000503 \\
.00000624\end{array}$ & $\begin{array}{r}71 \\
86 \\
103 \\
121 \\
142\end{array}$ & $\begin{array}{r}0.00000105 \\
.00000136 \\
.00000174 \\
.00000218 \\
.00000271\end{array}$ & $\begin{array}{l}31 \\
38 \\
44 \\
53 \\
61\end{array}$ \\
\hline $\begin{array}{r}0.040 \\
.042 \\
.044 \\
.046 \\
.048\end{array}$ & $\begin{array}{r}0.00000021 \\
.00000026 \\
.00000033 \\
.00000041 \\
.00000051\end{array}$ & $\begin{array}{r}5 \\
7 \\
8 \\
10 \\
12\end{array}$ & $\begin{array}{r}0.00000766 \\
.00000931 \\
.00001122 \\
.00001339 \\
.00001588\end{array}$ & $\begin{array}{l}165 \\
191 \\
217 \\
249 \\
280\end{array}$ & $\begin{array}{r}0.00000332 \\
.00000404 \\
.00000487 \\
.00000581 \\
.00000690\end{array}$ & $\begin{array}{r}72 \\
83 \\
94 \\
109 \\
122\end{array}$ \\
\hline $\begin{array}{l}0.050 \\
.052 \\
.054 \\
.056 \\
.058\end{array}$ & $\begin{array}{r}0.00000063 \\
.00000076 \\
.00000092 \\
.00000110 \\
.00000131\end{array}$ & $\begin{array}{l}13 \\
16 \\
18 \\
21 \\
25\end{array}$ & 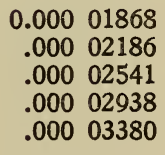 & $\begin{array}{l}318 \\
355 \\
397 \\
442 \\
490\end{array}$ & $\begin{array}{r}0.00000812 \\
.00000950 \\
.00001104 \\
.00001276 \\
.00001468\end{array}$ & $\begin{array}{l}138 \\
154 \\
172 \\
192 \\
213\end{array}$ \\
\hline $\begin{array}{l}0.060 \\
.062 \\
.064 \\
.066 \\
.068\end{array}$ & $\begin{array}{r}0.00000156 \\
.00000183 \\
.00000215 \\
.00000251 \\
.00000291\end{array}$ & $\begin{array}{l}27 \\
32 \\
36 \\
40 \\
45\end{array}$ & $\begin{array}{r}0.00003870 \\
.00004410 \\
.00005006 \\
.00005660 \\
.00006375\end{array}$ & $\begin{array}{l}540 \\
596 \\
654 \\
715 \\
781\end{array}$ & $\begin{array}{r}0.00001681 \\
.00001915 \\
.00002174 \\
.00002457 \\
.00002769\end{array}$ & $\begin{array}{l}234 \\
259 \\
283 \\
312 \\
339\end{array}$ \\
\hline $\begin{array}{r}0.070 \\
.072 \\
.074 \\
.076 \\
.078\end{array}$ & $\begin{array}{r}0.00000336 \\
.00000387 \\
.00000444 \\
.00000507 \\
.00000577\end{array}$ & $\begin{array}{l}51 \\
57 \\
63 \\
70 \\
78\end{array}$ & 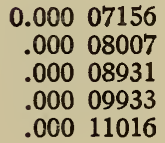 & $\begin{array}{r}851 \\
924 \\
1002 \\
1083 \\
1169\end{array}$ & 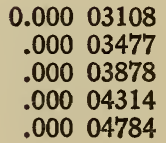 & $\begin{array}{l}369 \\
401 \\
436 \\
470 \\
509\end{array}$ \\
\hline
\end{tabular}

(For use with Formulas (8), (9), (45), (76), (77), and (78)) 
TABLE XV-Continued

\begin{tabular}{|c|c|c|c|c|c|c|}
\hline $\begin{array}{c}q \\
\text { or } q_{1}\end{array}$ & $\begin{array}{c}q-\frac{1}{2} \\
\text { or } q_{1}-\frac{1}{2}\end{array}$ & $\Delta$ & $\varepsilon$ & $\Delta$ & $\log _{10}(1+\varepsilon)$ & $\Delta$ \\
\hline $\begin{array}{r}0.080 \\
.082 \\
.084 \\
.086 \\
.088\end{array}$ & $\begin{array}{r}0.00000655 \\
.00000741 \\
.00000836 \\
.00000941 \\
.00001055\end{array}$ & $\begin{array}{r}86 \\
95 \\
105 \\
114 \\
126\end{array}$ & 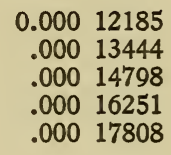 & $\begin{array}{l}1259 \\
1354 \\
1453 \\
1557 \\
1666\end{array}$ & $\begin{array}{r}0.00005293 \\
.00005838 \\
.00006426 \\
.00007057 \\
.00007733\end{array}$ & $\begin{array}{l}545 \\
588 \\
631 \\
676 \\
724\end{array}$ \\
\hline $\begin{array}{r}0.090 \\
.092 \\
.094 \\
.096 \\
.098\end{array}$ & $\begin{array}{r}0.00001181 \\
.00001318 \\
.00001468 \\
.00001630 \\
.00001807\end{array}$ & $\begin{array}{l}137 \\
150 \\
162 \\
177 \\
193\end{array}$ & $\begin{array}{r}0.00019474 \\
.00021253 \\
.00023152 \\
.00025174 \\
.00027324\end{array}$ & $\begin{array}{l}1779 \\
1899 \\
2022 \\
2150 \\
2285\end{array}$ & 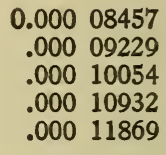 & $\begin{array}{l}772 \\
825 \\
878 \\
937 \\
988\end{array}$ \\
\hline $\begin{array}{r}0.100 \\
.101 \\
.102 \\
.103 \\
.104\end{array}$ & $\begin{array}{r}0.00002000 \\
.00002102 \\
.00002208 \\
.00002318 \\
.00002433\end{array}$ & $\begin{array}{l}102 \\
106 \\
110 \\
115 \\
119\end{array}$ & $\begin{array}{r}0.00029609 \\
.00030803 \\
.00032033 \\
.00033299 \\
.00034602\end{array}$ & $\begin{array}{l}1194 \\
1230 \\
1266 \\
1303 \\
1340\end{array}$ & 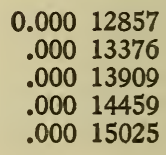 & $\begin{array}{l}519 \\
533 \\
550 \\
566 \\
582\end{array}$ \\
\hline $\begin{array}{r}0.105 \\
.106 \\
.107 \\
.108 \\
.109\end{array}$ & $\begin{array}{r}0.00002552 \\
.00002675 \\
.00002804 \\
.00002938 \\
.00003076\end{array}$ & $\begin{array}{l}123 \\
129 \\
134 \\
138 \\
144\end{array}$ & $\begin{array}{r}0.00035942 \\
.00037321 \\
.00038731 \\
.00040196 \\
.00041694\end{array}$ & $\begin{array}{l}1379 \\
1410 \\
1465 \\
1498 \\
1539\end{array}$ & 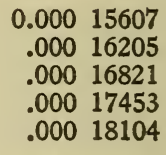 & $\begin{array}{l}598 \\
616 \\
632 \\
651 \\
668\end{array}$ \\
\hline $\begin{array}{r}0.110 \\
.111 \\
.112 \\
.113 \\
.114\end{array}$ & $\begin{array}{r}0.00003220 \\
.00003369 \\
.00003523 \\
.00003683 \\
.00003849\end{array}$ & $\begin{array}{l}149 \\
154 \\
160 \\
166 \\
172\end{array}$ & $\begin{array}{r}0.00043233 \\
.00044814 \\
.00046438 \\
.00048105 \\
.00049816\end{array}$ & $\begin{array}{l}1581 \\
1624 \\
1667 \\
1711 \\
1756\end{array}$ & 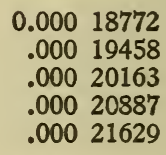 & $\begin{array}{l}686 \\
705 \\
724 \\
742 \\
762\end{array}$ \\
\hline $\begin{array}{l}0.115 \\
.116 \\
.117 \\
.118 \\
.119\end{array}$ & $\begin{array}{r}0.00004021 \\
.00004199 \\
.00004383 \\
.00004574 \\
.00004770\end{array}$ & $\begin{array}{l}178 \\
184 \\
191 \\
196 \\
204\end{array}$ & $\begin{array}{r}0.00051572 \\
.00053374 \\
.00055222 \\
.00057117 \\
.00059060\end{array}$ & $\begin{array}{l}1802 \\
1848 \\
1895 \\
1943 \\
1992\end{array}$ & $\begin{array}{r}0.00022391 \\
.00023174 \\
.00023976 \\
.00024799 \\
.00025642\end{array}$ & $\begin{array}{l}783 \\
802 \\
823 \\
843 \\
865\end{array}$ \\
\hline $\begin{array}{r}0.120 \\
.121 \\
.122 \\
.123 \\
.124\end{array}$ & $\begin{array}{r}0.00004974 \\
.00005184 \\
.00005402 \\
.00005628 \\
.00005860\end{array}$ & $\begin{array}{l}210 \\
218 \\
226 \\
232 \\
240\end{array}$ & $\begin{array}{r}0.00061052 \\
.00063093 \\
.00065184 \\
.00067327 \\
.00069522\end{array}$ & $\begin{array}{l}2041 \\
2091 \\
2143 \\
2195 \\
2247\end{array}$ & 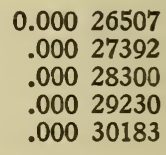 & $\begin{array}{l}885 \\
908 \\
930 \\
953 \\
975\end{array}$ \\
\hline $\begin{array}{r}0.125 \\
.126 \\
.127 \\
.128 \\
.129\end{array}$ & $\begin{array}{r}0.00006100 \\
.00006348 \\
.00006603 \\
.00006868 \\
.00007140\end{array}$ & $\begin{array}{l}248 \\
255 \\
265 \\
272 \\
280\end{array}$ & $\begin{array}{r}0.00071769 \\
.00074070 \\
.00076425 \\
.00078835 \\
.00081301\end{array}$ & $\begin{array}{l}2301 \\
2355 \\
2410 \\
2466 \\
2523\end{array}$ & 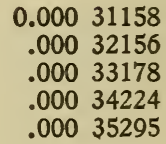 & $\begin{array}{r}998 \\
1022 \\
1046 \\
1071 \\
1094\end{array}$ \\
\hline
\end{tabular}


TABLE XV-Continued

\begin{tabular}{|c|c|c|c|c|c|c|}
\hline $\operatorname{or}^{q} q_{1}$ & $\begin{array}{c}q-\frac{1}{2} \\
\text { or } q_{1}-\frac{l_{1}}{2}\end{array}$ & $\triangle$ & $\varepsilon$ & $\Delta$ & $\log _{10}(1+\varepsilon)$ & $\Delta$ \\
\hline $\begin{array}{l}0.130 \\
.131 \\
.132 \\
.133 \\
.134\end{array}$ & $\begin{array}{r}0.00007420 \\
.00007710 \\
.00008009 \\
.00008317 \\
.00008634\end{array}$ & $\begin{array}{l}290 \\
299 \\
308 \\
317 \\
327\end{array}$ & $\begin{array}{r}0.00083824 \\
.00086405 \\
.00089044 \\
.00091742 \\
.00094501\end{array}$ & $\begin{array}{l}2581 \\
2639 \\
2698 \\
2759 \\
2820\end{array}$ & $\begin{array}{r}0.00036389 \\
.00037509 \\
.00038654 \\
.00039825 \\
.00041021\end{array}$ & $\begin{array}{l}1120 \\
1145 \\
1171 \\
1196 \\
1224\end{array}$ \\
\hline $\begin{array}{r}0.135 \\
.136 \\
.137 \\
.138 \\
.139\end{array}$ & $\begin{array}{r}0.00008961 \\
.00009297 \\
.00009644 \\
.00010001 \\
.00010368\end{array}$ & $\begin{array}{l}336 \\
347 \\
357 \\
367 \\
378\end{array}$ & $\begin{array}{r}0.00097321 \\
.001 \\
.00202 \\
.00103147 \\
.00106155 \\
.00109228\end{array}$ & $\begin{array}{l}2881 \\
2945 \\
3012 \\
3073 \\
3138\end{array}$ & $\begin{array}{r}0.00042245 \\
.00043496 \\
.00044773 \\
.00046078 \\
.00047411\end{array}$ & $\begin{array}{l}1251 \\
1277 \\
1305 \\
1333 \\
1362\end{array}$ \\
\hline $\begin{array}{r}0.140 \\
.141 \\
.142 \\
.143 \\
.144\end{array}$ & 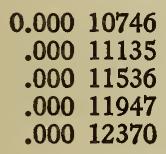 & $\begin{array}{l}389 \\
401 \\
411 \\
423 \\
435\end{array}$ & 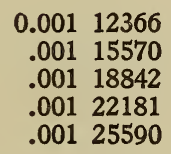 & $\begin{array}{l}3204 \\
3272 \\
3339 \\
3409 \\
3479\end{array}$ & $\begin{array}{r}0.00048773 \\
.00050162 \\
.00051582 \\
.00053030 \\
.00054509\end{array}$ & $\begin{array}{l}1389 \\
1420 \\
1448 \\
1479 \\
1509\end{array}$ \\
\hline $\begin{array}{l}0.145 \\
.146 \\
.147 \\
.148 \\
.149\end{array}$ & $\begin{array}{r}0.00012805 \\
.00013253 \\
.00013712 \\
.00014185 \\
.00014670\end{array}$ & $\begin{array}{l}448 \\
459 \\
473 \\
485 \\
498\end{array}$ & 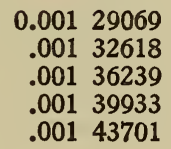 & $\begin{array}{l}3549 \\
3621 \\
3694 \\
3768 \\
3842\end{array}$ & $\begin{array}{r}0.00056018 \\
.00057557 \\
.00059128 \\
.00060730 \\
.00062364\end{array}$ & $\begin{array}{l}1539 \\
1571 \\
1602 \\
1634 \\
1666\end{array}$ \\
\hline 0.150 & 0.00015168 & & 0.00147543 & & 0.00064030 & \\
\hline
\end{tabular}

Tables XV and XVI are reproduced from Nagaoka's paper; see footnote, page $\mathrm{r} 2$. 


\section{TABLE XVI}

Values of $\epsilon_{1}$ and $-\epsilon_{1}^{\prime}$ with Argument $q_{1}$

$$
\begin{aligned}
\epsilon_{1} & =32 q_{1}^{3}-40 q_{1}{ }^{4}+48 q_{1}{ }^{5} \ldots . . \\
-\epsilon_{1}{ }^{\prime} & =8 q_{1}^{2}-\epsilon_{1} .
\end{aligned}
$$

\begin{tabular}{|c|c|c|c|c|}
\hline $\mathbf{q}_{1}$ & $\epsilon_{1}$ & $\Delta$ & $-\epsilon_{1}^{\prime}$ & $\Delta$ \\
\hline $\begin{array}{r}0.0100 \\
.0099 \\
.0098 \\
.0097 \\
.0096\end{array}$ & $\begin{array}{r}0.00003160 \\
.00003067 \\
.00002975 \\
.00002886 \\
.00002797\end{array}$ & $\begin{array}{l}93 \\
92 \\
89 \\
89 \\
86\end{array}$ & $\begin{array}{r}0.00076840 \\
.00075341 \\
.00073857 \\
.00072386 \\
.00070931\end{array}$ & $\begin{array}{l}1499 \\
1484 \\
1471 \\
1455 \\
1442\end{array}$ \\
\hline $\begin{array}{l}0.0095 \\
.0094 \\
.0093 \\
.0092 \\
.0091\end{array}$ & $\begin{array}{r}0.00002711 \\
.00002627 \\
.00002544 \\
.00002463 \\
.00002385\end{array}$ & $\begin{array}{l}84 \\
83 \\
81 \\
78 \\
78\end{array}$ & $\begin{array}{r}0.00069489 \\
.00068061 \\
.00066648 \\
.00065249 \\
.00063863\end{array}$ & $\begin{array}{l}1428 \\
1413 \\
1399 \\
1386 \\
1370\end{array}$ \\
\hline $\begin{array}{l}0.0090 \\
.0089 \\
.0088 \\
.0087 \\
.0086\end{array}$ & $\begin{array}{r}0.00002307 \\
.00002231 \\
.00002157 \\
.00002084 \\
.00002013\end{array}$ & $\begin{array}{l}76 \\
74 \\
73 \\
71 \\
69\end{array}$ & $\begin{array}{r}0.00062493 \\
.00061137 \\
.00059795 \\
.00058468 \\
.00057155\end{array}$ & $\begin{array}{l}1356 \\
1342 \\
1327 \\
1313 \\
1299\end{array}$ \\
\hline $\begin{array}{r}0.0085 \\
.0084 \\
.0083 \\
.0082 \\
.0081\end{array}$ & $\begin{array}{r}0.00001944 \\
.00001877 \\
.00001810 \\
.00001746 \\
.00001684\end{array}$ & $\begin{array}{l}67 \\
67 \\
64 \\
62 \\
62\end{array}$ & $\begin{array}{r}0.00055856 \\
.00054571 \\
.00053302 \\
.00052046 \\
.00050804\end{array}$ & $\begin{array}{l}1285 \\
1269 \\
1256 \\
1242 \\
1226\end{array}$ \\
\hline $\begin{array}{l}0.0080 \\
.0079 \\
.0078 \\
.0077 \\
.0076\end{array}$ & 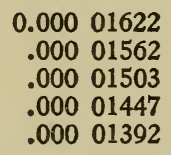 & $\begin{array}{l}60 \\
59 \\
56 \\
55 \\
55\end{array}$ & $\begin{array}{r}0.00049578 \\
.00048366 \\
.00047169 \\
.00045985 \\
.00044816\end{array}$ & $\begin{array}{l}1212 \\
1197 \\
1184 \\
1169 \\
1153\end{array}$ \\
\hline $\begin{array}{r}0.0075 \\
.0074 \\
.0073 \\
.0072 \\
.0071\end{array}$ & 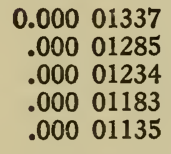 & $\begin{array}{l}52 \\
51 \\
51 \\
48 \\
47\end{array}$ & $\begin{array}{r}0.00043663 \\
.00042523 \\
.00041398 \\
.00040289 \\
.00039193\end{array}$ & $\begin{array}{l}1140 \\
1125 \\
1109 \\
1096 \\
1081\end{array}$ \\
\hline $\begin{array}{r}0.0070 \\
.0069 \\
.0068 \\
.0067 \\
.0066\end{array}$ & $\begin{array}{r}0.00001088 \\
.00001042 \\
.00000997 \\
.00000954 \\
.00000912\end{array}$ & $\begin{array}{l}46 \\
45 \\
43 \\
42 \\
40\end{array}$ & $\begin{array}{r}0.00038112 \\
.00037046 \\
.00035995 \\
.00034958 \\
.00033936\end{array}$ & $\begin{array}{l}1066 \\
1051 \\
1037 \\
1022 \\
1008\end{array}$ \\
\hline $\begin{array}{r}0.0065 \\
.0064 \\
.0063 \\
.0062 \\
.0061\end{array}$ & $\begin{array}{r}0.00000872 \\
.00000832 \\
.00000794 \\
.00000757 \\
.00000720\end{array}$ & $\begin{array}{l}40 \\
38 \\
37 \\
37 \\
34\end{array}$ & $\begin{array}{r}0.00032928 \\
.00031936 \\
.00030958 \\
.00029995 \\
.00029048\end{array}$ & $\begin{array}{l}992 \\
978 \\
963 \\
947 \\
934\end{array}$ \\
\hline
\end{tabular}

(For use with Formulas (9) and (9a)) 
TABLE XVI-Continued

\begin{tabular}{|c|c|c|c|c|}
\hline$q_{1}$ & $\epsilon_{1}$ & $\Delta$ & $-\epsilon_{1}^{\prime}$ & $\Delta$ \\
\hline $\begin{array}{l}0.0060 \\
.0059 \\
.0058 \\
.0057 \\
.0056\end{array}$ & $\begin{array}{r}0.00000686 \\
.00000652 \\
.00000619 \\
.00000589 \\
.00000558\end{array}$ & $\begin{array}{l}34 \\
33 \\
30 \\
31 \\
30\end{array}$ & $\begin{array}{r}0.00028114 \\
.00027196 \\
.00026293 \\
.00025403 \\
.00024530\end{array}$ & $\begin{array}{l}918 \\
903 \\
890 \\
873 \\
858\end{array}$ \\
\hline $\begin{array}{l}0.0055 \\
.0054 \\
.0053 \\
.0052 \\
.0051\end{array}$ & $\begin{array}{r}0.00000528 \\
.00000501 \\
.00000473 \\
.00000447 \\
.00000421\end{array}$ & $\begin{array}{l}27 \\
28 \\
26 \\
26 \\
24\end{array}$ & $\begin{array}{r}0.00023672 \\
.00022827 \\
.00021999 \\
.00021185 \\
.00020387\end{array}$ & $\begin{array}{l}845 \\
828 \\
814 \\
798 \\
784\end{array}$ \\
\hline $\begin{array}{l}0.0050 \\
.0049 \\
.0048 \\
.0047 \\
.0046\end{array}$ & $\begin{array}{r}0.00000397 \\
.00000374 \\
.00000352 \\
.00000330 \\
.00000309\end{array}$ & $\begin{array}{l}23 \\
22 \\
22 \\
21 \\
19\end{array}$ & $\begin{array}{r}0.00019603 \\
.00018834 \\
.00018080 \\
.00017342 \\
.00016619\end{array}$ & $\begin{array}{l}769 \\
754 \\
738 \\
723 \\
709\end{array}$ \\
\hline $\begin{array}{l}0.0045 \\
.0044 \\
.0043 \\
.0042 \\
.0041\end{array}$ & $\begin{array}{r}0.00000290 \\
.00000272 \\
.00000253 \\
.00000236 \\
.00000220\end{array}$ & $\begin{array}{l}18 \\
19 \\
17 \\
16 \\
16\end{array}$ & 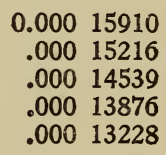 & $\begin{array}{l}694 \\
677 \\
663 \\
648 \\
632\end{array}$ \\
\hline $\begin{array}{l}0.0040 \\
.0039 \\
.0038 \\
.0037 \\
.0036\end{array}$ & $\begin{array}{r}0.00000204 \\
.00000189 \\
.00000175 \\
.00000161 \\
.00000148\end{array}$ & $\begin{array}{l}15 \\
14 \\
14 \\
13 \\
12\end{array}$ & 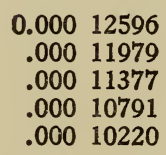 & $\begin{array}{l}617 \\
602 \\
586 \\
571 \\
556\end{array}$ \\
\hline $\begin{array}{r}0.0035 \\
.0034 \\
.0033 \\
.0032 \\
.0031\end{array}$ & $\begin{array}{r}0.00000136 \\
.00000125 \\
.00000114 \\
.00000105 \\
.00000095\end{array}$ & $\begin{array}{r}11 \\
11 \\
9 \\
10 \\
9\end{array}$ & $\begin{array}{r}0.00009664 \\
.00009123 \\
.00008598 \\
.00008087 \\
.00007593\end{array}$ & $\begin{array}{l}541 \\
525 \\
511 \\
494 \\
479\end{array}$ \\
\hline $\begin{array}{l}0.0030 \\
.0029 \\
.0028 \\
.0027 \\
.0026\end{array}$ & $\begin{array}{r}0.00000086 \\
.00000078 \\
.000 .00070 \\
.00000063 \\
.00000056\end{array}$ & $\begin{array}{l}8 \\
8 \\
7 \\
7 \\
6\end{array}$ & 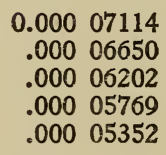 & $\begin{array}{l}464 \\
448 \\
433 \\
417 \\
402\end{array}$ \\
\hline $\begin{array}{l}0.0025 \\
.0024 \\
.0023 \\
.0022 \\
.0021\end{array}$ & $\begin{array}{r}0.00000050 \\
.00000044 \\
.00000039 \\
.00000034 \\
.00000030\end{array}$ & $\begin{array}{l}6 \\
5 \\
5 \\
4 \\
4\end{array}$ & 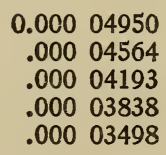 & $\begin{array}{l}386 \\
371 \\
355 \\
340 \\
324\end{array}$ \\
\hline $\begin{array}{r}0.0020 \\
.0019 \\
.0018 \\
.0017 \\
.0016\end{array}$ & $\begin{array}{r}0.00000026 \\
.00000022 \\
.00000019 \\
.00000016 \\
.00000013\end{array}$ & $\begin{array}{l}4 \\
3 \\
3 \\
3 \\
2\end{array}$ & $\begin{array}{r}0.00003174 \\
.00002866 \\
.00002573 \\
.00002296 \\
.00002035\end{array}$ & $\begin{array}{l}308 \\
293 \\
277 \\
261 \\
246\end{array}$ \\
\hline
\end{tabular}


TABLE XVI--Continued

\begin{tabular}{c|c|c||c|c}
\hline$q_{1}$ & $\epsilon_{1}$ & $\Delta$ & $-e_{1}^{\prime}$ & $\Delta$ \\
\hline & & & & \\
0.0015 & 0.00000011 & 2 & 0.00001789 & 230 \\
.0014 & .00000009 & 2 & .00001559 & 214 \\
.0013 & .00000007 & 1 & .00001345 & 199 \\
.0012 & .00000006 & 2 & .00001146 & 182 \\
.0011 & .00000004 & 1 & .00000964 & 167 \\
& & 1 & 0.00000797 & 151 \\
0.0010 & 0.00000003 & 0 & .00000646 & 136 \\
.0009 & .00000002 & 1 & .00000510 & 119 \\
.0008 & .00000002 & 0 & .00000391 & 104 \\
.0007 & .00000001 & 1 & .00000287 & 87 \\
.0006 & .00000001 & & 0.00000200 & 72 \\
& & & .00000128 & 56 \\
0.0005 & 0.00000000 & & .00000072 & 40 \\
.0004 & .00000000 & & .00000032 & 24 \\
.0003 & .00000000 & & .00000008 &. \\
.0002 & .00000000 & & & \\
.0001 & .00000000 & & & \\
\hline
\end{tabular}

TABLE XVII

Coefficients of the Hypergeometric Series in Formula (18)

\begin{tabular}{c|c|c|c}
\hline Series & $a_{1}$ & $a_{2}$ & $a_{3}$ \\
$F\left(\frac{1}{12}, \frac{5}{12}, \frac{1}{2}, \frac{J-1}{J}\right)$ & 0.0694444 & 0.0355260 & 0.0238485 \\
$F\left(-\frac{1}{12}, \frac{7}{12}, \frac{1}{2}, \frac{J-1}{J}\right)$ & -0.092222 & -0.0470358 & -0.0310523 \\
$F\left(\frac{5}{12}, \frac{13}{12}, \frac{3}{2}, \frac{J-1}{J}\right)$ & 0.3009259 & 0.1776300 & 0.1260562 \\
$F\left(\frac{7}{12}, \frac{11}{12}, \frac{3}{2}, \frac{J-1}{J}\right)$ & 0.3564814 & 0.2163645 & 0.1552615 \\
\hline
\end{tabular}




\section{TABLE XVIII}

Showing the Location and Magnitude of the Positive and Negative Maxima and the Positions of the Roots of the Coefficients in Gray's and Searle and Airey's Formulas

(For use in Formulas (40), (43), and (56))

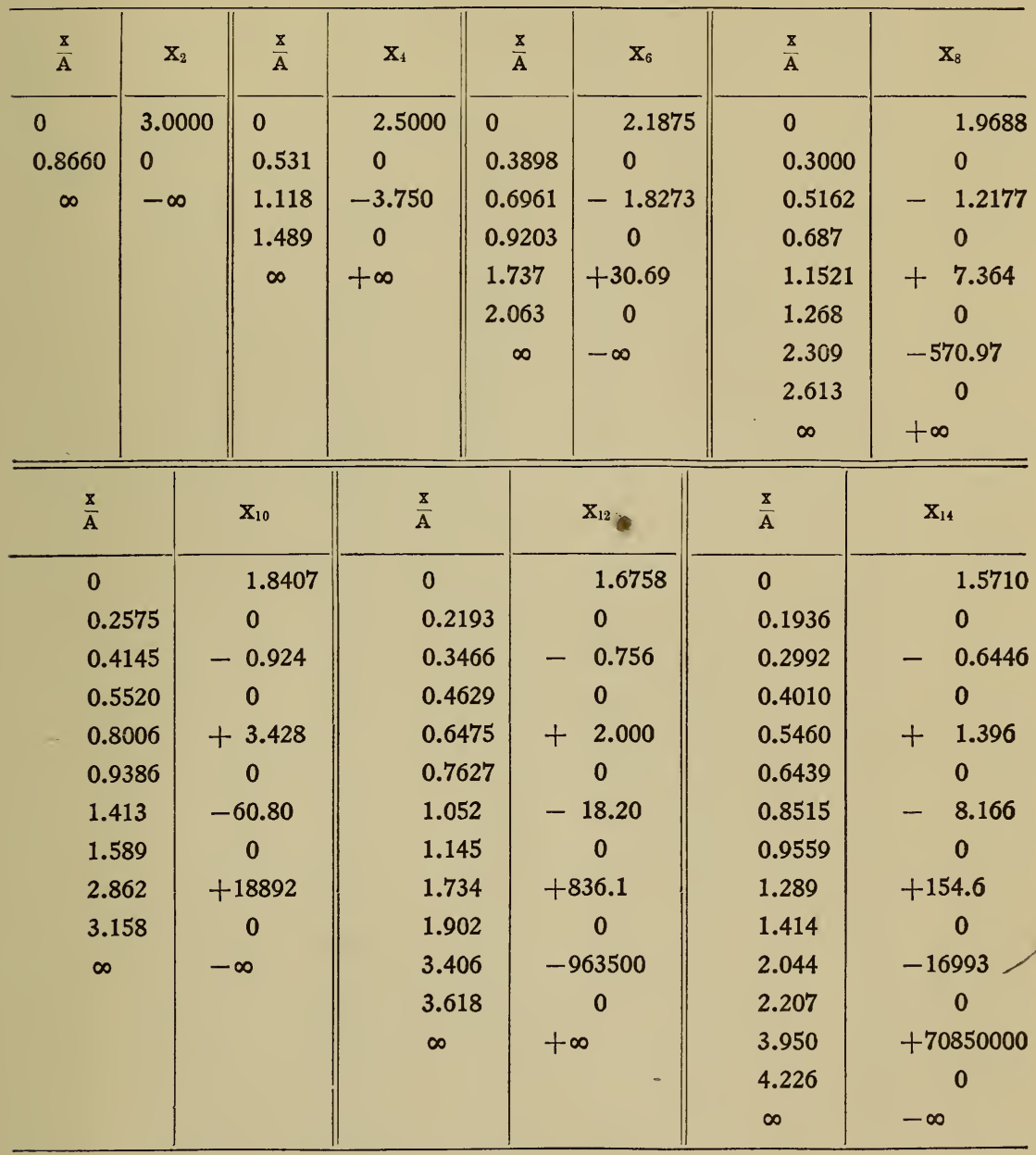

The function $X_{2 n}$ has $n$ roots, between which values it makes oscillations of ever-increasing amplitude, and for values of $\frac{x}{A}$ greater than the largest root the function increases rapidly without limit. The functions $L_{2 n}$ have the same form as $X_{2 n}, \frac{l}{a}$ being the variable instead of $\frac{x}{A}$. 
TABLE XIX

Values of Coefficients in Gray's and Searle and Airey's Formulas

(For use in Formulas (40), (43), and (56))

\begin{tabular}{|c|c|c|c|c|c|c|c|}
\hline$\frac{\mathrm{X}}{\mathrm{A}}$ & $x_{2}$ & $\mathbf{x}_{4}$ & $\mathbf{X}_{6}$ & $\mathbf{X}_{8}$ & $\mathbf{X}_{10}$ & $\mathbf{X}_{12}$ & $\mathbf{X}_{14}$ \\
\hline 0.0 & +3.000 & $+\quad 2.500$ & $+\quad 2.188$ & $+\quad 1.969$ & +1.841 & +1.676 & +1.571 \\
\hline 0.1 & 2.960 & 2.400 & 2.015 & 1.712 & 1.494 & 1.234 & +1.032 \\
\hline 0.2 & 2.840 & 2.106 & 1.521 & 1.017 & +0.618 & +0.216 & -0.073 \\
\hline 0.3 & 2.640 & 1.632 & $+\quad 0.780$ & $+\quad 0.090$ & -0.355 & -0.635 & -0.645 \\
\hline 0.4 & 2.360 & 1.002 & $-\quad 0.090$ & $-\quad 0.764$ & -0.874 & -0.596 & -0.0093 \\
\hline 0.5 & 2.000 & $+\quad 0.250$ & - $\quad 0.938$ & $-\quad 1.203$ & -0.526 & +0.483 & +1.208 \\
\hline 0.6 & 1.560 & - $\quad 0.580$ & $-\quad 1.577$ & $-\quad 0.909$ & +0.760 & +1.793 & +1.000 \\
\hline 0.7 & 1.040 & $-\quad 1.438$ & $-\quad 1.814$ & $+\quad 0.228$ & 2.467 & +1.662 & -3.175 \\
\hline 0.8 & +0.440 & $-\quad 2.262$ & $-\quad 1.452$ & $+\quad 2.207$ & 3.423 & -1.733 & -7.231 \\
\hline 0.9 & -0.240 & $-\quad 2.976$ & $-\quad 0.335$ & +4.606 & +1.924 & -8.748 & -6.811 \\
\hline 1.0 & -1.000 & 3.500 & +1.688 & $+\quad 6.719$ & - 3.878 & -16.46 & +10.48 \\
\hline 1.1 & -1.840 & $-\quad 3.750$ & 4.673 & 7.240 & & & \\
\hline 1.2 & -2.760 & $-\quad 3.606$ & 8.589 & $+\quad 4.509$ & -31.72 & +22.27 & +119.6 \\
\hline 1.3 & -3.760 & $-\quad 2.976$ & 13.28 & $-\quad 3.595$ & & & \\
\hline 1.4 & -4.840 & $-\quad 1.734$ & 18.44 & -19.49 & -60.66 & & +29.1 \\
\hline 1.5 & -6.000 & $+\quad 0.250$ & +23.56 & $-\quad 46.24$ & -48.96 & +765.7 & -486.5 \\
\hline 1.6 & -7.240 & $+\quad 3.114$ & 27.90 & -89.42 & & & \\
\hline 1.7 & -8.560 & 7.008 & 30.46 & -137.4 & +151.0 & +818.1 & \\
\hline 1.8 & -9.960 & 12.09 & 29.83 & -205.2 & & & \\
\hline 1.9 & -11.44 & 18.53 & 24.50 & -285.9 & & +21.8 & \\
\hline 2.0 & -13.00 & +26.50 & +12.19 & -375.0 & +1591 & -1969 & -16740 \\
\hline 2.1 & -14.64 & 36.55 & - $\quad 9.64$ & -464.9 & & & \\
\hline 2.2 & -16.36 & 47.80 & -44.09 & -538.3 & 4059 & -14090 & -1840 \\
\hline 2.3 & -18.16 & 62.54 & -104.9 & -570.9 & & & \\
\hline 2.4 & -20.04 & 77.61 & -166.3 & -535.5 & & & \\
\hline 2.5 & -22.00 & +96.24 & -263.4 & -386.7 & 10908 & -80050 & \\
\hline 2.6 & -24.04 & 117.6 & -390.4 & -64.4 & & & +658400 \\
\hline 2.7 & -26.16 & 142.2 & -559.0 & +505.9 & & & \\
\hline 2.8 & -28.36 & 169.0 & -801.2 & 1433 & 18390 & -222400 & \\
\hline 2.9 & -30.64 & 201.3 & -1039.1 & 2833 & & & \\
\hline 3.0 & -33.00 & +236.5 & -1370.3 & +4869 & +15797 & -509200 & 19132000 \\
\hline 3.25 & -38.25 & +343.1 & -2553 & +14118 & & & \\
\hline 3.5 & $-46,00$ & +480.2 & -4414 & +33030 & -146970 & -893400 & 33670000 \\
\hline 3.75 & -53.25 & +653.1 & -7215 & +68410 & & +265600 & \\
\hline 4.0 & -61.00 & +866.5 & -11286 & +130400 & $-1.229 \times 10^{6}$ & $+6.625 \times 10^{6}$ & 59080000 \\
\hline 4.25 & -69.25 & & & & & & -16530000 \\
\hline 4.5 & -77.00 & +1440.3 & -24956 & +399000 & $-5.683 \times 10^{6}$ & $+5.972 \times 10^{7}$ & $-4.172 \times 10^{3}$ \\
\hline 5.0 & -97.00 & +2252.5 & -49810 & +1038700 & $-2.007 \times 10^{7}$ & $+3.463 \times 10^{8}$ & $-4.855 \times 10^{9}$ \\
\hline
\end{tabular}

This table used in conjunction with the preceding should make it possible to investigate the convergence of Gray's or Searle and Airey's formula in any given case. It will also facilitate calculations by these formulas when $\frac{x}{A}$ has one of the values included in the table. This table gives also the values of the $L_{2 n}$ coefficients if $\frac{l}{a}$ be taken as argument in place of $\frac{x}{A}$. 


\section{TABLE XX}

Nagaoka's Table of Values of the Correction Factor for the Ends $K$, as a Function of the Angle $\theta=\tan ^{-1} \frac{2 a}{b}$

(For use in Formula (75))

\begin{tabular}{|c|c|c|c|c|c|c|c|}
\hline$\theta$ & $K$ & $\Delta_{1}$ & $\triangle_{2}$ & $\theta$ & $\kappa$ & $\triangle_{1}$ & $\triangle_{2}$ \\
\hline $\begin{array}{l}0^{\circ} \\
1 \\
2 \\
3 \\
4\end{array}$ & 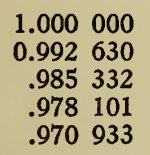 & $\begin{array}{l}-7370 \\
-7298 \\
-7231 \\
-.7168 \\
-7109\end{array}$ & $\begin{array}{r}72 \\
67 \\
63 \\
60 \\
56\end{array}$ & $\begin{array}{l}45^{\circ} \\
46 \\
47 \\
48 \\
49\end{array}$ & $\begin{array}{r}0.688423 \\
.680764 \\
.673010 \\
.665154 \\
.657190\end{array}$ & $\begin{array}{l}-7659 \\
-7754 \\
-7856 \\
-7964 \\
-8079\end{array}$ & $\begin{array}{l}-95 \\
-102 \\
=108 \\
-115 \\
-120\end{array}$ \\
\hline $\begin{array}{l}5 \\
6 \\
7 \\
8 \\
9\end{array}$ & $\begin{array}{r}0.963825 \\
.956771 \\
.949770 \\
.942815 \\
.935906\end{array}$ & $\begin{array}{l}-7053 \\
-7001 \\
-6955 \\
-6910 \\
-6870\end{array}$ & $\begin{array}{r}52 \\
47 \\
44 \\
40 \\
37\end{array}$ & $\begin{array}{l}50 \\
51 \\
52 \\
53 \\
54\end{array}$ & 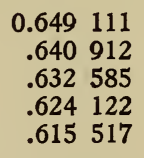 & $\begin{array}{l}-8199 \\
-8327 \\
-8463 \\
-8605 \\
-8757\end{array}$ & $\begin{array}{l}-128 \\
-136 \\
-142 \\
-152 \\
-160\end{array}$ \\
\hline $\begin{array}{l}10 \\
11 \\
12 \\
13 \\
14\end{array}$ & $\begin{array}{r}0.929036 \\
.922203 \\
.915404 \\
.908635 \\
.901 \quad 893\end{array}$ & $\begin{array}{l}-6833 \\
-6799 \\
-6769 \\
-6742 \\
-6718\end{array}$ & $\begin{array}{r}34 \\
30 \\
27 \\
24 \\
19\end{array}$ & $\begin{array}{l}55 \\
56 \\
57 \\
58 \\
59\end{array}$ & $\begin{array}{r}0.606760 \\
.597843 \\
.588757 \\
.579492 \\
.570037\end{array}$ & $\begin{array}{l}-8917 \\
-9086 \\
-9265 \\
-9455 \\
-9655\end{array}$ & $\begin{array}{l}-169 \\
-179 \\
-190 \\
-\quad 200 \\
-\quad 214\end{array}$ \\
\hline $\begin{array}{l}15 \\
16 \\
17 \\
18 \\
19\end{array}$ & 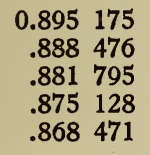 & $\begin{array}{l}-6699 \\
-6681 \\
-6667 \\
-6657 \\
-6649\end{array}$ & $\begin{array}{r}18 \\
14 \\
10 \\
8 \\
4\end{array}$ & $\begin{array}{l}60 \\
61 \\
62 \\
63 \\
64\end{array}$ & 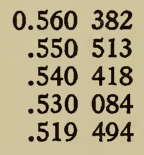 & $\begin{array}{l}9869 \\
-10095 \\
-10334 \\
-10590 \\
-10860\end{array}$ & $\begin{array}{l}-226 \\
-239 \\
-256 \\
-270 \\
-288\end{array}$ \\
\hline $\begin{array}{l}20 \\
21 \\
22 \\
23 \\
24\end{array}$ & 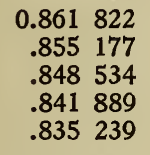 & $\begin{array}{l}-6645 \\
-6643 \\
-6645 \\
-6650 \\
-6659\end{array}$ & $\begin{array}{l}+\quad 2 \\
-\quad 2 \\
-\quad 5 \\
=\quad 9 \\
-10\end{array}$ & $\begin{array}{l}65 \\
66 \\
67 \\
68 \\
69\end{array}$ & 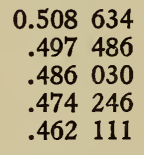 & $\begin{array}{l}-11148 \\
-11456 \\
-11784 \\
-12135 \\
-12511\end{array}$ & $\begin{array}{l}-308 \\
-328 \\
-351 \\
-376 \\
-403\end{array}$ \\
\hline $\begin{array}{l}25 \\
26 \\
27 \\
28 \\
29\end{array}$ & $\begin{array}{r}0.828580 \\
.821511 \\
.815226 \\
.808524 \\
.801801\end{array}$ & $\begin{array}{l}-6669 \\
-6685 \\
-6702 \\
-6723 \\
-6747\end{array}$ & $\begin{array}{l}-16 \\
-17 \\
-21 \\
=24 \\
-28\end{array}$ & $\begin{array}{l}70 \\
71 \\
72 \\
72 \\
74\end{array}$ & $\begin{array}{r}0.449600 \\
.436686 \\
.423337 \\
.409521 \\
.395199\end{array}$ & $\begin{array}{l}-12914 \\
-13349 \\
-13816 \\
-14322 \\
-14871\end{array}$ & $\begin{array}{l}-435 \\
-467 \\
-506 \\
-549 \\
-597\end{array}$ \\
\hline $\begin{array}{l}30 \\
31 \\
32 \\
33 \\
34\end{array}$ & 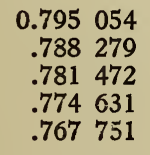 & $\begin{array}{l}-6775 \\
-6807 \\
-6841 \\
-6880 \\
-6921\end{array}$ & $\begin{array}{l}-32 \\
-34 \\
-39 \\
-41 \\
-46\end{array}$ & $\begin{array}{l}75 \\
76 \\
77 \\
78 \\
79\end{array}$ & $\begin{array}{r}0.380328 \\
.364860 \\
.348739 \\
.331901 \\
.314272\end{array}$ & $\begin{array}{l}-15468 \\
-16121 \\
-16838 \\
-17629 \\
-18510\end{array}$ & $\begin{array}{r}-653 \\
-717 \\
-791 \\
-881 \\
-985\end{array}$ \\
\hline $\begin{array}{l}35 \\
36 \\
37 \\
38 \\
39\end{array}$ & $\begin{array}{r}0.760830 \\
.753863 \\
.746846 \\
.739775 \\
.732647\end{array}$ & $\begin{array}{l}-6967 \\
-7017 \\
-7071 \\
-7128 \\
-7189\end{array}$ & $\begin{array}{l}-50 \\
-54 \\
-57 \\
-61 \\
-67\end{array}$ & $\begin{array}{l}80 \\
81 \\
82 \\
83 \\
84\end{array}$ & 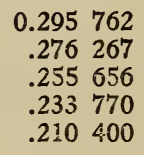 & $\begin{array}{l}-19495 \\
-20611 \\
-21886 \\
-23370 \\
-25128\end{array}$ & $\begin{array}{l}-1116 \\
-1275 \\
-1484 \\
-1758 \\
-2144\end{array}$ \\
\hline $\begin{array}{l}40 \\
41 \\
42 \\
43 \\
44\end{array}$ & $\begin{array}{r}0.725458 \\
.718202 \\
.710875 \\
.703473 \\
.695990\end{array}$ & $\begin{array}{l}7256 \\
-7327 \\
-7402 \\
-7483 \\
-7567\end{array}$ & $\begin{array}{l}-71 \\
-75 \\
-81 \\
-84 \\
-92\end{array}$ & $\begin{array}{l}85 \\
86 \\
87 \\
88 \\
89\end{array}$ & $\begin{array}{r}0.185272 \\
.158000 \\
.128003 \\
.094299 \\
.054835\end{array}$ & $\begin{array}{l}-27272 \\
-29997 \\
-33704 \\
-39464 \\
-54835\end{array}$ & $\begin{array}{l}-2725 \\
-\quad 3707 \\
-5760 \\
-15371\end{array}$ \\
\hline
\end{tabular}




\section{TABLE XXI}

Nagaoka's Table of Values of the End Correction $K$ as Function of the Ratio $\frac{\text { Diameter }}{\text { Length }}$

For use in Formula (75))

\begin{tabular}{|c|c|c|c|c|c|c|c|}
\hline$\frac{\text { Diameter }}{\text { Length }}$ & $K$ & $\triangle_{1}$ & $\triangle_{2}$ & $\frac{\text { Diameter }}{\text { Length }}$ & $K$ & $\Delta_{1}$ & $\triangle_{2}$ \\
\hline 0.00 & 1.000000 & -4231 & +24 & 0.45 & 0.833723 & -3160 & +21 \\
\hline .01 & .995769 & -4207 & 26 & .46 & .830563 & -3139 & 22 \\
\hline .02 & 991562 & -4181 & 24 & .47 & $.827 \quad 424$ & -3117 & 21 \\
\hline .03 & .987381 & -4157 & 25 & .48 & .824307 & -3096 & 21 \\
\hline .04 & .983224 & -4132 & 25 & .49 & .821211 & -3075 & 21 \\
\hline 0.05 & 0.979092 & -4107 & +25 & 0.50 & $0.818 \quad 136$ & -3054 & +21 \\
\hline .06 & .974985 & -4082 & 26 & .51 & .815082 & -3033 & 21 \\
\hline .07 & 970903 & -4056 & 24 & .52 & .812049 & -3012 & 21 \\
\hline .08 & 966847 & -4032 & 24 & .53 & .809037 & -2991 & 20 \\
\hline .09 & .962815 & -4008 & 26 & .54 & .806046 & -2971 & 21 \\
\hline 0.10 & 0.958807 & -3982 & +25 & 0.55 & 0.803075 & -2950 & +20 \\
\hline .11 & .954825 & -3957 & 24 & .56 & .800125 & -2930 & 20 \\
\hline .12 & .950868 & -3933 & 23 & .57 & $.797 \quad 195$ & -2910 & 20 \\
\hline .13 & 946935 & -3910 & 26 & .58 & $.794 \quad 285$ & -2890 & 20 \\
\hline .14 & .943025 & -3884 & 27 & .59 & .791395 & -2870 & 20 \\
\hline 0.15 & 0.939141 & -3857 & +23 & 0.60 & $0.788 \quad 525$ & -2850 & +19 \\
\hline .16 & .935284 & -3834 & 23 & .61 & .785675 & -2831 & 19 \\
\hline .17 & .931450 & -3811 & 26 & .62 & .782844 & -2812 & 20 \\
\hline .18 & .927 639 & -3785 & 24 & .63 & .780032 & -2792 & 19 \\
\hline .19 & .923854 & -3761 & 24 & .64 & .777240 & -2773 & 19 \\
\hline 0.20 & 0.920093 & -3737 & +24 & 0.65 & $0.774 \quad 467$ & -2754 & +19 \\
\hline .21 & .916356 & -3713 & 24 & .66 & .771713 & -2735 & 19 \\
\hline .22 & .912643 & -3689 & 25 & .67 & .768978 & -2716 & 19 \\
\hline .23 & 908954 & -3664 & 23 & .68 & .766262 & -2697 & 18 \\
\hline .24 & .905290 & -3641 & 25 & .69 & .763565 & -2679 & 18 \\
\hline 0.25 & 0.901649 & -3616 & +23 & 0.70 & 0.760886 & -2661 & +18 \\
\hline .26 & .898033 & -3593 & 24 & .71 & $.758 \quad 225$ & -2643 & 19 \\
\hline .27 & .894440 & -3569 & 23 & .72 & .755582 & -2624 & 17 \\
\hline .28 & .890871 & -3546 & 24 & .73 & .752958 & -2607 & 18 \\
\hline .29 & .887325 & -3522 & 24 & .74 & .750351 & -2589 & 18 \\
\hline 0.30 & 0.883803 & -3498 & +22 & 0.75 & $0.747 \quad 762$ & -2571 & +17 \\
\hline .31 & .880305 & -3476 & 24 & .76 & $.745 \quad 191$ & -2554 & 17 \\
\hline .32 & .876829 & -3452 & 23 & .77 & .742637 & -2537 & 18 \\
\hline .33 & .873377 & -3429 & 23 & .78 & .740100 & -2519 & 17 \\
\hline .34 & .869948 & -3406 & 22 & .79 & .737581 & -2502 & 16 \\
\hline 0.35 & 0.866542 & -3384 & +24 & 0.80 & 0.735079 & -2486 & +19 \\
\hline .36 & .863158 & -3360 & 22 & .81 & .732593 & -2467 & 16 \\
\hline .37 & .859 799 & -3338 & 23 & .82 & .730126 & -2451 & 16 \\
\hline .38 & .856461 & -3315 & 22 & .83 & .727675 & -2435 & 16 \\
\hline .39 & .853146 & -3293 & 23 & .84 & .725240 & -2419 & 17 \\
\hline 0.40 & 0.849853 & -3270 & +22 & 0.85 & 0.722821 & -2402 & +16 \\
\hline .41 & .846583 & -3248 & 23 & .86 & .720419 & -2386 & 16 \\
\hline .42 & .843335 & -3225 & 21 & .87 & .718033 & -2370 & 15 \\
\hline .43 & .840110 & -3204 & 21 & .88 & .715663 & -2355 & 16 \\
\hline .44 & .836906 & -3183 & 23 & .89 & .713308 & -2339 & 17 \\
\hline
\end{tabular}


TABLE XXI-Continued

\begin{tabular}{|c|c|c|c|c|c|c|c|c|}
\hline$\frac{\text { Diameter }}{\text { Length }}$ & $K$ & $\triangle_{1}$ & $\triangle_{2}$ & $\frac{\text { Diameter }}{\text { Length }}$ & $K$ & $\triangle_{1}$ & $\triangle_{2}$ & $\triangle_{3}$ \\
\hline 0.90 & 0.710969 & -2322 & +14 & 2.50 & 0.471865 & -9292 & +405 & \\
\hline .91 & .708647 & -2308 & 16 & 2.60 & .452573 & -8887 & 378 & \\
\hline .92 & .706339 & -2292 & 15 & 2.70 & .453686 & -8509 & 355 & \\
\hline .93 & .704047 & -2277 & 16 & 2.80 & $.445 \quad 177$ & -8154 & 330 & \\
\hline .94 & .701770 & -2261 & 14 & 2.90 & .437023 & -7824 & 312 & \\
\hline 0.95 & 0.699509 & -2247 & +15 & 3.00 & 0.429199 & -7512 & +293 & \\
\hline .96 & .697262 & -2232 & 15 & 3.10 & .421687 & -7219 & 275 & \\
\hline .97 & .695030 & -2217 & 15 & 3.20 & .414468 & -6944 & 260 & \\
\hline .98 & .692813 & -2202 & 14 & 3.30 & .407524 & -6684 & 245 & \\
\hline .99 & .690611 & -2188 & 14 & 3.40 & .400840 & -6439 & 230 & \\
\hline 1.00 & $0.688 \quad 423$ & -10726 & +344 & 3.50 & 0.394401 & -6209 & +220 & \\
\hline 1.05 & .677697 & -10382 & 330 & 3.60 & .388192 & -5989 & 207 & \\
\hline 1.10 & .667315 & -10052 & 316 & 3.70 & .382203 & -5782 & 195 & \\
\hline 1.15 & .657263 & -9736 & 303 & 3.80 & .376421 & -5587 & 186 & \\
\hline 1.20 & .647527 & -9433 & 290 & 3.90 & .370834 & -5401 & 174 & \\
\hline 1.25 & 0.638094 & -9143 & +278 & 4.00 & 0.365433 & -5227 & +168 & \\
\hline 1.30 & .628951 & -8865 & 266 & 4.10 & .360206 & -5059 & 161 & \\
\hline 1.35 & .620086 & -8599 & 255 & 4.20 & .355147 & -4898 & 152 & \\
\hline 1.40 & .611487 & -8343 & 244 & 4.30 & .350249 & -4746 & 141 & \\
\hline 1.45 & .603144 & -8099 & 236 & 4.40 & .345503 & -4605 & 138 & \\
\hline 1.50 & 0.595045 & -7863 & +224 & 4.50 & 0.340898 & -4467 & +134 & \\
\hline 1.55 & .587182 & -7639 & 215 & 4.60 & .336431 & -4333 & 125 & \\
\hline 1.60 & .579543 & -7424 & 208 & 4.70 & .332098 & -4208 & 118 & \\
\hline 1.65 & .572119 & -7216 & 198 & 4.80 & .327890 & -4090 & 115 & \\
\hline 1.70 & .564903 & -7018 & 190 & 4.90 & .323800 & -3975 & 102 & \\
\hline 1.75 & 0.557885 & -6828 & +184 & 5.00 & 0.319825 & -18321 & +2227 & -397 \\
\hline 1.80 & .551057 & -6644 & 176 & 5.50 & .301504 & -16094 & 1830 & -306 \\
\hline 1.85 & .544413 & -6468 & 170 & 6.00 & .285410 & -14264 & 1524 & -241 \\
\hline 1.90 & .537945 & -6298 & 161 & 6.50 & .271146 & -12740 & 1283 & -193 \\
\hline 1.95 & .531647 & -6137 & 154 & 7.00 & .258406 & -11457 & 1090 & -153 \\
\hline 2.00 & 0.525510 & -11809 & +580 & 7.50 & 0.246949 & -10367 & +937 & -127 \\
\hline 2.10 & .513701 & -11229 & 539 & 8.00 & .236582 & -9430 & $810^{\circ}$ & -104 \\
\hline 2.20 & .502472 & -10690 & 499 & 8.50 & .227152 & -8620 & 706 & -86 \\
\hline 2.30 & .491782 & -10191 & 465 & 9.00 & .218532 & -7914 & 620 & \\
\hline \multirow[t]{2}{*}{2.40} & .481591 & -9726 & 434 & 9.50 & .210618 & -7294 & & \\
\hline & & & & 10.00 & 0.203324 & & & \\
\hline
\end{tabular}

In the last part of this table several errors in the fifth and sixth places of decimals have been corrected. 


\section{TABLE XXII}

Functions for Calculating Resistance and Inductance of Straight, Cylindrical Wires with Varying Frequency (sec. 10)

\begin{tabular}{|c|c|c|c|c|c|c|c|c|c|c|c|c|}
\hline$x$ & $\frac{W}{Y}$ & $\triangle_{1}$ & $\triangle_{2}$ & $\frac{\mathrm{X}}{2} \quad \frac{\mathrm{W}}{\mathrm{Y}}$ & $\triangle_{1}$ & $\triangle_{2}$ & $\frac{Z}{Y}$ & $\triangle_{1}$ & $\triangle_{2}$ & $\frac{4}{\mathrm{X}} \quad \frac{Z}{\mathrm{Y}}$ & $\Delta_{1}$ & $\triangle_{2}$ \\
\hline 0.0 & $\infty$ & $\ldots$. & $\ldots$ & 1.00000 & & +1 & 0. & +2500 & $\ldots$ & 1.00000 & & \\
\hline .1 & 20.00000 & $\ldots$. & $\ldots$ & 1.00000 & + & +2 & 0.02500 & 2500 & $\ldots$ & 1.00000 & 0 & -2 \\
\hline .2 & 10.00020 & $\ldots$ & $\ldots$ & 1.00001 & & +6 & 0.05000 & 2500 & -1 & 1.00000 & - & -3 \\
\hline .3 & 6.66693 & $\ldots$. & $\ldots$ & 1.00004 & & +10 & 0.07500 & 2499 & & 0.99998 & - & -5 \\
\hline .4 & 5.00065 & $\cdots$ & $\cdots$ & 1.00013 & 19 & +16 & 0.09999 & 2499 & -2 & 0.99993 & -10 & -8 \\
\hline 0.5 & 4.00128 & $\ldots$ & $\ldots$ & 1.00032 & +35 & +22 & 0.12498 & +2497 & -3 & 0.99984 & -18 & -11 \\
\hline .6 & 3.33557 & $\ldots$. & $\ldots$ & 1.00067 & 57 & +31 & 0.14995 & 2494 & -4 & 0.99966 & -29 & -14 \\
\hline .7 & 2.86069 & $\ldots$. & $\ldots$ & 1.00124 & 88 & +40 & 0.17489 & 2490 & -7 & 0.99937 & -43 & -20 \\
\hline .8 & 2.50530 & $\ldots$ & $\ldots$ & 1.00212 & 128 & +51 & 0.19979 & 2483 & -10 & 0.99894 & -64 & -25 \\
\hline 9 & 2.22978 & $\cdots$ & $\cdots$ & 1.00340 & 179 & +60 & 0.22462 & 2473 & -12 & 0.99830 & -89 & -31 \\
\hline 1.0 & 2.01038 & $\theta^{-1}$ & $\ldots$ & 1.00519 & +239 & +74 & 0.24935 & +2461 & -18 & 0.99 & -120 & -36 \\
\hline 1.1 & 1.83196 & $\ldots$. & $\ldots$ & 1.00758 & 313 & +86 & 0.27396 & 2443 & -21 & 0.99621 & -156 & -43 \\
\hline 1.2 & 1.68451 & $\ldots$. & $\ldots$ & $1-01071$ & 399 & 100 & 0.29839 & 2422 & -27 & 0.99465 & -199 & -50 \\
\hline 1.3 & 1.56108 & $\ldots$ & $\ldots$ & 1.01470 & 499 & 114 & 0.32261 & 2395 & -34 & 0.99266 & -249 & -57 \\
\hline 1.4 & 1.45670 & $\cdots$ & $\cdots$ & 1.01969 & 613 & 128 & 0.34656 & 2361 & -41 & 0.99017 & -306 & -63 \\
\hline 1.5 & 1.36776 & $\cdots$ & $\cdots$ & 1.02582 & +741 & 141 & 17 & +2320 & -48 & 0.9 & -369 & -69 \\
\hline 1.6 & 1.29154 & $\ldots$. & $\ldots$ & 1.03323 & 882 & 153 & 0.39337 & 2272 & -56 & 0.98342 & -438 & -76 \\
\hline 1.7 & 1.22594 & $\ldots$ & $\ldots$ & 1.04205 & 1035 & 165 & 0.41609 & 2216 & -64 & 0.97904 & -514 & -81 \\
\hline 1.8 & 1.16934 & $\ldots$ & $\ldots$ & 1.05240 & 1200 & 176 & 0.43825 & 2152 & -72 & 0.97390 & -595 & -86 \\
\hline 1.9 & 1.12042 & $\cdots$ & $\cdots$ & 1.06440 & 1376 & 183 & 0.45977 & 2080 & -81 & 0.96795 & -681 & -89 \\
\hline 2.0 & 1.07816 & -3649 & +505 & 1.07816 & 1559 & 192 & & +1999 & -90 & 0.9 & -770 & -92 \\
\hline 2.1 & 1.04167 & -3144 & 442 & 1.09375 & 1751 & 192 & 0.50056 & 1909 & -96 & 0.95343 & -862 & -92 \\
\hline 2.2 & 1.01023 & 2702 & 387 & 1.11126 & 1943 & 195 & 0.51965 & 1813 & -102 & 0.94482 & -954 & -91 \\
\hline 2.3 & 0.98321 & 2315 & 339 & 1.13069 & 2138 & 192 & 0.53778 & 1711 & -108 & 0.93527 & -1045 & -90 \\
\hline 2.4 & 0.96006 & 1976 & 297 & 1.15207 & 2330 & 188 & 0.55489 & 1603 & -113 & 0.92482 & -1135 & -86 \\
\hline 2.5 & 0.94030 & -1679 & +256 & 1.17538 & 2518 & 179 & 0.57092 & +1490 & -115 & 0.91347 & -1221 & -81 \\
\hline 2.6 & 0.92351 & 1423 & 224 & 1.20056 & 2697 & 170 & 0.58582 & 1375 & -116 & 0.90126 & -1301 & -73 \\
\hline 2.7 & 0.90928 & 1199 & 190 & 1.22753 & 2867 & 157 & 0.59957 & 1259 & -116 & 0.88825 & -1374 & -65 \\
\hline 2.8 & 0.89729 & 1009 & 162 & 1.25620 & 3024 & 142 & 0.61216 & 1143 & -114 & 0.87451 & -1439 & -56 \\
\hline 2.9 & 0.88720 & 847 & 136 & 1.28644 & 3166 & 128 & 0.62359 & 1029 & -111 & 0.86012 & -1495 & -47 \\
\hline 3.0 & 0.87873 & -711 & +114 & 1.31809 & +3293 & +109 & 0.63388 & +918 & -106 & 0.84517 & -1542 & -36 \\
\hline 3.1 & 0.87162 & 597 & 92 & 1.35102 & 3402 & +93 & 0.64306 & 812 & -100 & 0.82975 & -1578 & -25 \\
\hline 3.2 & 0.86565 & 505 & 75 & 1.38504 & 3495 & +76 & 0.65118 & 712 & -93 & 0.81397 & -1603 & -16 \\
\hline 3.3 & 0.86060 & 430 & 58 & 1.41999 & 3571 & +61 & 0.65830 & 619 & -87 & 0.79794 & -1619 & -6 \\
\hline 3.4 & 0.85630 & 372 & 46 & 1.45570 & 3632 & +44 & 0.66449 & 532 & -78 & 0.78175 & -1625 & +4 \\
\hline 3.5 & 0.85258 & -326 & +36 & 1.49202 & +3677 & +31 & 0.66981 & 454 & -70 & 0.76550 & -1621 & +11 \\
\hline 3.6 & 0.84932 & 290 & 24 & 1.52879 & 3708 & +19 & 0.67436 & 384 & -61 & 0.74929 & -1610 & +20 \\
\hline 3.7 & 0.84642 & 266 & 18 & 1.56587 & 3727 & +10 & 0.67820 & 323 & -55 & 0.73320 & -1590 & 26 \\
\hline 3.8 & 0.84376 & 248 & 13 & 1.60314 & 3737 & -1 & 0.58143 & 268 & -47 & 0.71729 & -1564 & 31 \\
\hline 3.9 & 0.84128 & 235 & 8 & 1.64051 & 3736 & -7 & 0.68411 & 221 & $-4 C$ & 0.70165 & -1533 & 36 \\
\hline
\end{tabular}


TABLE XXII-Continued

\begin{tabular}{|c|c|c|c|c|c|c|c|c|c|c|c|}
\hline x & $\frac{W}{Y}$ & $\triangle_{1}$ & $\triangle_{2}$ & $\frac{X}{2} \quad \frac{W}{Y}$ & $\triangle_{2}$ & $\frac{Z}{\bar{Y}}$ & $\triangle_{1}$ & $\triangle_{2}$ & $\frac{4}{\bar{X}} \quad \frac{Z}{\bar{Y}}$ & $\triangle_{1}$ & $\triangle_{2}$ \\
\hline 4.0 & 0.83893 & 227 & +5 & 1.67787 & $+3729-12$ & 0.68632 & +181 & -33 & 0.68632 & -1497 & +39 \\
\hline 4.1 & 0.83666 & 222 & 3 & 1.71516 & $3717-17$ & 0.68813 & 148 & -28 & 0.67135 & -1458 & 43 \\
\hline 4.2 & 0.83444 & 219 & +1 & 1.75233 & $3700-19$ & 0.68961 & 120 & -23 & 0.65677 & -1415 & 43 \\
\hline 4.3 & 0.83225 & 218 & 0 & 1.78933 & $3681-20$ & 0.69082 & 97 & -17 & 0.64262 & -1372 & 45 \\
\hline 4.4 & 0.83007 & 218 & 0 & 1.82614 & $3661-22$ & 0.69179 & 80 & -15 & 0.62890 & -1327 & 45 \\
\hline 4.5 & 0.82789 & -218 & +1 & 1.86275 & $+3639-20$ & 0.69259 & +65 & -12 & 0.61563 & -1282 & +45 \\
\hline 4.6 & 0.82571 & 217 & +1 & 1.89914 & $3619-21$ & 0.69324 & 53 & -8 & 0.60281 & -1237 & 45 \\
\hline 4.7 & 0.82354 & 216 & +1 & 1.93533 & $3598-19$ & 0.69377 & 45 & -6 & 0.59044 & -1192 & 43 \\
\hline 4.8 & 0.82138 & 215 & +1 & 1.97131 & $3579-17$ & 0.69422 & 39 & -4 & 0.57852 & -1149 & 43 \\
\hline 4.9 & 0.81923 & -214 & +2 & 2.00710 & $+3562-15$ & 0.69461 & 35 & -3 & 0.56703 & -1106 & 42 \\
\hline 5.0 & 0.81709 & -419 & +14 & 2.04272 & $+7081-45$ & 0.69496 & +62 & -6 & 0.55597 & -2091 & +151 \\
\hline 5.2 & 0.81290 & 405 & 18 & 2.11353 & $7036-31$ & 0.69558 & 56 & -1 & 0.53506 & 1940 & 138 \\
\hline 5.4 & 0.80885 & 387 & 20 & 2.18389 & $7005-19$ & 0.69614 & 55 & 0 & 0.51566 & 1802 & 124 \\
\hline 5.6 & 0.80498 & 367 & 22 & 2.25393 & $6987-8$ & 0.69669 & 55 & +1 & 0.49764 & 1678 & 112 \\
\hline 5.8 & 0.80131 & 345 & 23 & 2.32380 & $6979 \quad 0$ & 0.69725 & 56 & -1 & 0.48086 & 1566 & 101 \\
\hline 6.0 & 0.79786 & -322 & +21 & 2.39359 & $+6979+4$ & 0.69781 & +55 & -1 & 0.46521 & -1465 & +91 \\
\hline 6.2 & 0.79464 & 301 & 21 & 2.46338 & 6983 & 0.69836 & 54 & -2 & 0.45056 & 1374 & 82 \\
\hline 6.4 & 0.79163 & 280 & 19 & 2.53321 & 6992 & 0.69891 & 52 & -3 & 0.43682 & 1292 & 74 \\
\hline 6.6 & 0.78883 & 261 & 17 & 2.60313 & 6999 & 0.69942 & 49 & -3 & 0.4 & 1218 & 68 \\
\hline 6.8 & 0.78621 & 244 & 16 & 2.67312 & 7007 & 0.69991 & 46 & -4 & 0.41171 & 1150 & 62 \\
\hline 7.0 & 0.78377 & -228 & +13 & 2.74319 & $+7015+6$ & 0.70037 & +42 & -3 & 0.40021 & -1088 & +57 \\
\hline 7.2 & 0.78149 & 215 & 13 & 2.81334 & 7021 & 0.70 & 39 & 4 & 0.38933 & 1031 & 52 \\
\hline 7.4 & 0.77934 & 202 & 12 & 2.88355 & 7026 & 0.70118 & 35 & 3 & 0.37902 & 979 & 49 \\
\hline 7.6 & 0.77731 & 190 & 11 & 2.95380 & 7031 & 0.70154 & 32 & 3 & 0.36923 & 930 & 45 \\
\hline 7.8 & 0.77541 & 179 & 9 & 3.02411 & $7034+1$ & 0.70185 & 29 & -2 & 0.35992 & 885 & 42 \\
\hline 8.0 & 0.77361 & -170 & +8 & 3.09445 & $+7035+3$ & 0.70214 & +27 & -2 & 0.35107 & -843 & +39 \\
\hline 8.2 & 0.77191 & 162 & 8 & 3.16480 & $7038+1$ & 0.70241 & 25 & -2 & 0.34263 & 804 & 36 \\
\hline 8.4 & 0.77028 & 154 & 7 & 3.23518 & $7039+1$ & 0.70265 & 23 & -2 & 0.33460 & 768 & 34 \\
\hline 8.6 & 0.76874 & 147 & 7 & 3.30557 & $7040+1$ & 0.70288 & 20 & -1 & 0.32692 & 734 & 32 \\
\hline 8.8 & 0.76727 & 140 & 6 & 3.37597 & $041+1$ & 0.70308 & 19 & -1 & 0.31958 & 702 & 30 \\
\hline 9.0 & 0.76586 & -134 & +6 & 3.44638 & $+7042+1$ & 0.70327 & +18 & -2 & 0.31257 & -672 & +28 \\
\hline 9.2 & 0.76452 & 128 & 5 & 3.51680 & $7043-1$ & 0.70345 & 16 & 1 & 0.30585 & 644 & 26 \\
\hline 9.4 & 0.76324 & 123 & 6 & 3.58723 & $7043+2$ & 0.70362 & 15 & 1 & 0.29941 & 617 & 25 \\
\hline 9.6 & 0.76201 & 117 & 4 & 3.65766 & $7045+4$ & 0.70377 & 14 & 1 & 0.29324 & 593 & 23 \\
\hline 9.8 & 0.76084 & -113 & +4 & 3.72812 & $7046+2$ & 0.70391 & +13 & -1 & 0.28731 & -569 & +21 \\
\hline 10.0 & 0.75971 & -261 & +24 & 3.79857 & $+17620+3$ & 0.70405 & +30 & -4 & 0.28162 & -1330 & +120 \\
\hline 10.5 & 0.75710 & 237 & 21 & 3.97477 & 17623 & 0.70435 & 26 & 4 & 0.26832 & 1210 & 104 \\
\hline 11.0 & 0.75473 & 216 & 19 & 4.15100 & 17627 & 0.70461 & 22 & 3 & 0.25622 & 1106 & 91 \\
\hline 11.5 & 0.75257 & 197 & 16 & 4.32727 & 17631 & 0.70483 & 19 & 2 & 0.24516 & 1015 & 80 \\
\hline 12.0 & 0.75060 & 181 & 15 & 4.50358 & 17635 & 0.70503 & 17 & -2 & 0.23501 & 935 & 71 \\
\hline 12.5 & 0.74879 & -166 & +13 & 4.67993 & $+17638+3$ & 0.70520 & +15 & -1 & 0.22567 & -863 & +64 \\
\hline 13.0 & 0.74712 & 154 & 11 & 4.85631 & 17641 & 0.70535 & 14 & 2 & 0.21703 & 800 & 57 \\
\hline 13.5 & 0.74559 & 142 & 10 & 5.03272 & 17643 & 0.70549 & 12 & 1 & 0.20903 & 743 & 51 \\
\hline 14.0 & 0.74416 & 132 & 9 & 5.20915 & 17645 & 0.70561 & 11 & 2 & 0.20160 & 692 & 46 \\
\hline 14.5 & 0.74284 & -123 & +8 & 5.38560 & $17648+2$ & 0.70572 & +9 & -1 & 0.19468 & -646 & +40 \\
\hline
\end{tabular}


TABLE XXII-Continued

\begin{tabular}{|c|c|c|c|c|c|c|c|c|c|c|c|c|}
\hline$z$ & $\frac{W}{Y}$ & $\Delta_{1}$ & $\Delta_{2}$ & $\frac{\mathbb{Z}}{2} \frac{\mathrm{W}}{\mathrm{Y}}$ & $\triangle_{1}$ & $\Delta_{2}$ & $\frac{Z}{\bar{Y}}$ & $\Delta_{1}$ & $\Delta_{2}$ & $\frac{4}{\bar{X}} \quad \frac{Z}{\bar{Y}}$ & $\triangle_{1}$ & $\Delta_{2}$ \\
\hline 15.0 & 0.74161 & -222 & +27 & 5.56208 & +35301 & +6 & 0.70581 & +16 & -3 & 0.18822 & -1172 & +137 \\
\hline 16.0 & 0.73939 & 195 & 22 & 5.91509 & 35307 & 5 & 0.70597 & 13 & 2 & 0.17649 & 1035 & 114 \\
\hline 17.0 & 0.73743 & 173 & 19 & 6.26817 & 35312 & 5 & 0.70611 & 11 & 1 & 0.16614 & 921 & 97 \\
\hline 18.0 & 0.73570 & 154 & 16 & 6.62129 & 35317 & 4 & 0.70622 & 10 & 2 & 0.15694 & 824 & 82 \\
\hline 19.0 & 0.73416 & 139 & 13 & 6.97446 & 35321 & 3 & 0.70632 & 8 & -1 & 0.14870 & 742 & 70 \\
\hline 20.0 & 0.73277 & -125 & +11 & 7.32767 & +35324 & +3 & 0.70640 & 7 & -1 & 0.14128 & -672 & +61 \\
\hline 21.0 & 0.73151 & 114 & 10 & 7.68091 & 35327 & 2 & 0.70646 & 6 & 1 & 0.13456 & 611 & 53 \\
\hline 22.0 & 0.73038 & 104 & 9 & 8.03418 & 35329 & 2 & 0.70652 & 5 & 0 & 0.12846 & 558 & 46 \\
\hline 23.0 & 0.72935 & 95 & 8 & 8.38748 & 35331 & 2 & 0.70657 & 5 & 1 & 0.12288 & 511 & 41 \\
\hline 24.0 & 0.72840 & 87 & 7 & 8.74079 & 35333 & 2 & 0.70662 & 4 & -1 & 0.11777 & 470 & 36 \\
\hline 25.0 & 0.72753 & -80 & +5 & 9.09412 & +35335 & +2 & 0.70666 & +3 & 0 & 0.11307 & -434 & +32 \\
\hline 26.0 & 0.72673 & -143 & +20 & 9.44748 & +70674 & +5 & 0.70669 & 6 & -1 & 0.10872 & -776 & +103 \\
\hline 28.0 & 0.72530 & 123 & 15 & 10.15422 & 70679 & +4 & 0.70675 & 5 & -1 & 0.10096 & 672 & 84 \\
\hline 30.0 & 0.72407 & -108 & +13 & 10.86101 & +70683 & +3 & 0.70680 & +4 & -1 & 0.09424 & -589 & +69 \\
\hline 32.0 & 0.72299 & 95 & 11 & 11.56785 & 70686 & 3 & 0.70684 & 3 & .. & 0.08835 & 519 & 58 \\
\hline 34.0 & 0.72204 & 84 & 9 & 12.27471 & 70689 & 2 & 0.70687 & 2 & .. & 0.08316 & 462 & 49 \\
\hline 36.0 & 0.72120 & 75 & 8 & 12.98160 & 70691 & 2 & 0.70689 & 2 & .. & 0.07854 & 413 & 41 \\
\hline 38.0 & 0.72045 & 68 & 7 & 13.68852 & 70693 & 2 & 0.70691 & 2 & .. & 0.07441 & 372 & 35 \\
\hline 40.0 & 0.71977 & -61 & +6 & 14.39545 & +70695 & 1 & 0.70693 & +2 & .. & 0.07069 & -336 & +30 \\
\hline 42.0 & 0.71916 & 55 & 5 & 15.10240 & 70696 & 2 & 0.70695 & 1 & .. & 0.06733 & 306 & 27 \\
\hline 44.0 & 0.71861 & 50 & 4 & 15.80936 & 70698 & 1 & 0.70696 & 2 & .. & 0.06427 & 279 & 23 \\
\hline 46.0 & 0.71810 & 46 & 4 & 16.51634 & 70699 & 0 & 0.70698 & 1 & .. & 0.06148 & 256 & 20 \\
\hline 48.0 & 0.71764 & -43 & +3 & 17.22333 & +70699 & +1 & 0.70699 & +1 & .. & 0.05892 & -236 & +17 \\
\hline 50.0 & 0.71721 & -170 & +49 & 17.93032 & +353509 & +13 & 0.70700 & +3 & .. & 0.05656 & -942 & +269 \\
\hline 60.0 & 0.71551 & 121 & 30 & 21.46541 & 353522 & 8 & 0.70703 & 2 & .. & 0.04713 & 673 & 168 \\
\hline 70.0 & 0.71430 & 91 & 20 & 25.00063 & 353530 & 5 & 0.70705 & 1 & .. & 0.04040 & 505 & 112 \\
\hline 80.0 & 0.71340 & 70 & +14 & 28.53593 & 353535 & +3 & 0.70706 & 1 & .. & 0.03535 & 393 & 79 \\
\hline 90.0 & 0.71270 & -56 & & 32.07127 & 353538 & .. & 0.70707 & +1 & .. & 0.03142 & -314 & +55 \\
\hline 100.0 & 0.71213 & & & 35.60666 & & & 0.70708 & & & 0.02828 & & \\
\hline$\infty$ & 0.70711 & & & $\infty$ & & & 0.70711 & & & 0. & & \\
\hline
\end{tabular}




\section{TABLE XXIII}

Values of Limiting Change of Inductance with the Frequency

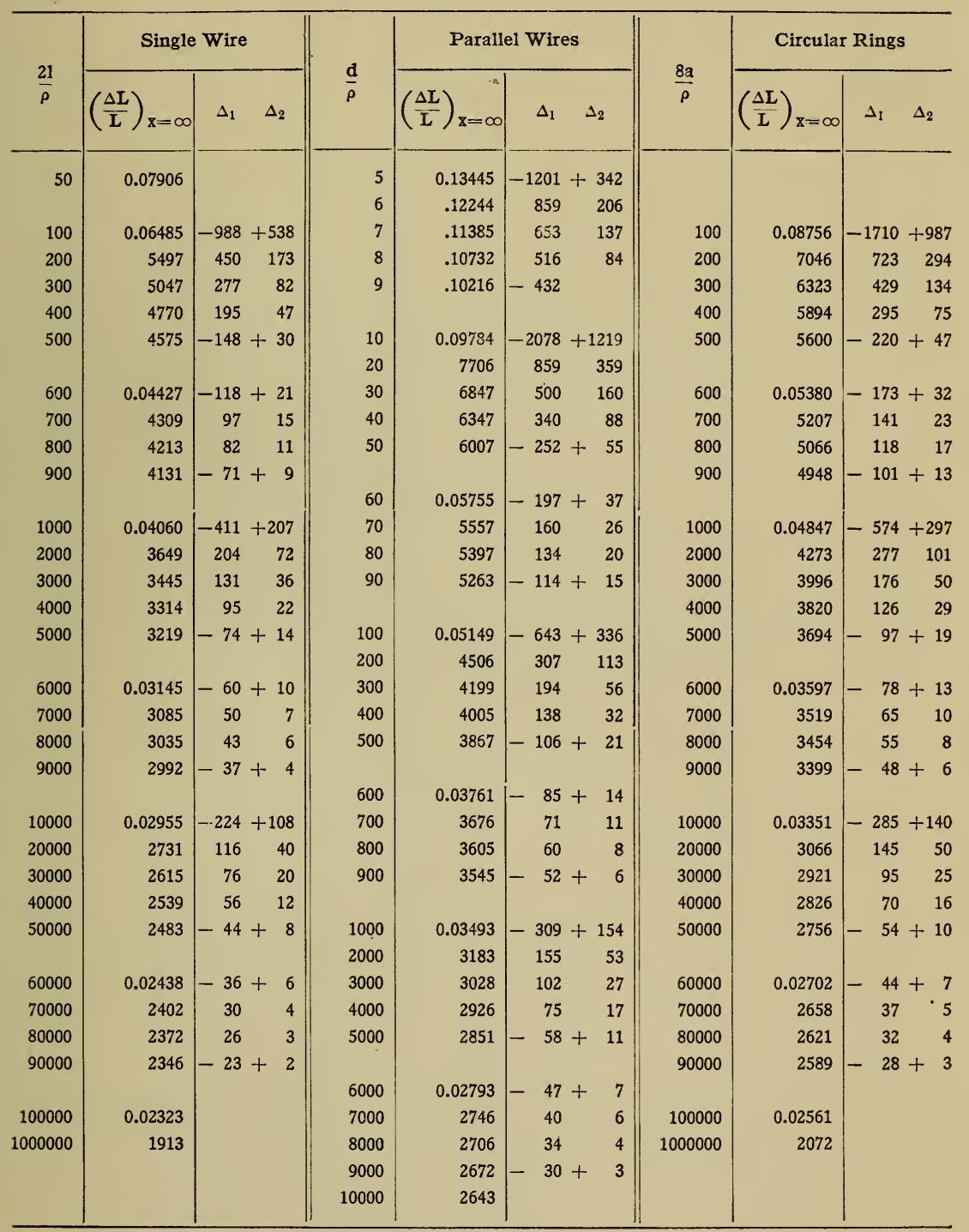




\section{TABLE XXIV}

Values of the Argument $x_{0}$ for Copper Wires $1 \mathrm{~mm}$ Radius and Conductivity $5.811 \times 10^{-4}$ c g. s. Units

\begin{tabular}{|c|c|c|c|c|c|}
\hline $\begin{array}{c}\text { f } \\
\text { cycles per } \\
\text { second }\end{array}$ & $x_{0}$ & $\stackrel{\lambda}{\lambda}$ meters & $\begin{array}{l}\text { f } \\
\text { cycles per } \\
\text { second }\end{array}$ & $x_{0}$ & $\stackrel{\lambda}{\text { meters }}$ \\
\hline 100 & 0.2142 & & 50000 & 4.790 & 6000 \\
\hline 200 & .3029 & $\ldots \ldots \ldots$. & 60000 & 5.247 & 5000 \\
\hline 300 & .3710 & $\ldots \ldots \ldots$ & 70000 & 5.667 & 4286 \\
\hline 400 & .4284 & $\ldots \ldots \ldots \ldots$ & 80000 & 6.058 & 3750 \\
\hline 500 & .4790 & $\ldots$ & 90000 & 6.426 & 3333 \\
\hline 600 & 0.5247 & & 100000 & 6.774 & 3000 \\
\hline 700 & .5667 & $\ldots+\ldots$ & 150000 & 8.296 & 2000 \\
\hline 800 & .6058 & $\ldots \ldots \ldots$ & 200000 & 9.579 & 1500 \\
\hline 900 & .6426 & $\ldots \ldots \ldots$ & 250000 & 10.710 & 1200 \\
\hline 1000 & .6774 & . & 300000 & 11.732 & 1000 \\
\hline 2000 & 0.9579 & & 333333 & 12.367 & 900 \\
\hline 3000 & 1.1732 & & 375000 & 13.117 & 800 \\
\hline 4000 & 1.3547 & & 428570 & 14.023 & 700 \\
\hline \multirow[t]{2}{*}{5000} & 1.5146 & & 500000 & 15.146 & 600 \\
\hline & & & 600000 & 16.592 & 500 \\
\hline 6000 & 1.6592 & & 700000 & 17.921 & 429 \\
\hline 7000 & 1.7921 & . & 750000 & 18.550 & 400 \\
\hline 8000 & 1.9158 & $\ldots$. & 800000 & 19.158 & 375 \\
\hline 9000 & 2.0321 & & 900000 & 20.321 & 333 \\
\hline ........ & ........ & .......... & 1000000 & 21.42 & 300 \\
\hline 10000 & 2.142 & 30000 & 1500000 & 26.23 & 200 \\
\hline 15000 & 2.623 & 20000 & 3000000 & 37.10 & 100 \\
\hline 20000 & 3.029 & 15000 & & & \\
\hline 30000 & 3.710 & 10000 & & & \\
\hline 40000 & 4.284 & 7500 & & & \\
\hline
\end{tabular}




\section{INDEX}

[Italicized page numbers refer to examples illustrating the formulas. Proper names are also italicized.]

A

Absolute formulas: Mutual inductance of coaxial circles, 6, 7, 9, 20, 21, 23; mutual inductance of concentric, coaxial solenoids, $64,73,78,91$; mutual inductance of coaxial solenoids, $64,69,73,89$; self-inductance of solenoids, II7, II8, I29, $1_{32}, r_{33}$, Table IV; mutual inductance of circle and solenoid, 99, Ioo, ro3, 106, 107, 108, 109; inductance of rectangle of rectangular section, I55.

Absolute invariant I7.

Adjacent conductors. See Linear conductors.

Airey. See Searle and Airey.

Ampere balance. See Current balance.

Amplitude of incomplete elliptic integrals, 64, Ioo.

Annular area, geometric mean distance of, I68, I69; geometric mean distance of point to area, I69.

Annulus. See Annular area.

Approximate formulas; inductance of solenoid of more than one layer, Cohen, I40, 150; inductance of circular ring, Kirchhoff, I IO, II4; mutual inductance of coaxial solenoids of equal length, 55, 78; inductance of coil of rectangular cross section, Maxwell, I35, I43; Perry I26, 143; mutual inductance of coaxial circles, Wiedemann, footnote $I 3$.

Arithmetical mean distance, I7I, I72.

Arithmetical mean square distance, I7I, I72.

Asymptotic formulas for $W, Y$, and $Z, 176$.

Attraction of coils. See Current balance.

Ayrton and Jones current balance, IO4.

Bar. See Rectangular bar.

Ber and bei functions, I74, 175 .

Bessel functions, I5, I74.

Bláthy, inductance of a ring, I 13 .

Breadth, equivalent of coil $38,47,48$.

Bromwich, I6, II I, II3.

Campbell, extension of Jones's formula, I00, rog; form for standard of mutual inductance, 108 .

Choice of formulas: Mutual inductance of coaxial circles, 19 ; mutual inductance of coaxial coils of rectangular section, 43; mutual inductance of coaxial solenoids, 73 , $83,84,86$; inductance of solenoids, 125 ; inductance of coils of rectangular cross section, I42.

Circles. See Coaxial circles, Circular areas, Circle and coaxial solenoid.

Circle and coaxial solenoid, mutual inductance of, 98-1 10.

Circular areas: Geometric mean distance of, 167 ; geometric mean distance of a point from, r69; geometric mean distance of two, r70; arithmetical mean distance of, $\mathrm{r} 7 \mathrm{r}$; arithmetical mean square distance of, I7I. 
Circular coils of rectangular cross section : Inductance of, $135-150$; choice of formulas, I42; mutual inductance of coaxial, 33-52; choice of formulas, 42.

Coaxial circles: Formulas for the inductance of, 6-32; choice of formulas, 19 ; summary of formulas, 19.

Coaxial coils of rectangular cross section: Formulas for the mutual inductance of, $33-52$; choice of formulas, 42.

Coaxial solenoids: Formulas for the mutual inductance of, 52-98; case where coils are not concentric, $59,64,73$; choice of formulas, $73,83,84,86$.

Coefficients: In Stefan's formula (90), I96; in hypergeometrical series of Mathy formula (18), 220; in formulas (40), (43), and (56), 221, 222.

Coffin, Absolute formula for mutual inductance of equal circles, $14,15,30$; coaxial solenoids, 73; inductance of solenoid, II 7, 129 ; derivation of Lorenz's formula, II8.

Cohen, absolute formula for the mutual inductance of coaxial solenoids $64,69,73$, $78,79,9 I$; correction of Wien's formula, III ; approximate formula for the inductance of solenoids of several layers, 140, 150 .

Complementary modulus, 8 , Io, II.

Complete elliptic integrals. See Elliptic integrals.

Concentric coaxial solenoids. See Coaxial solenoids.

Concentric conductors, inductance of, 158 ; with high frequency, 179.

Conductors. See Concentric conductors, linear conductors, rings, tubes, tapes, etc. Constant of Lorenz apparatus. See Lorenz apparatus.

Constants. See Tables of constants, etc.

Correction, of current sheet formulas for inductance of solenoids for windiug of round wire, 122, 127; for unequal distribution of current over cross section of coil, I40-142, I47-I49; of simple expression for toroidal coil, I25.

Correction factor. See End correction, correction, etc.

Cross section. See Coaxial coils of rectangular cross section, Circular coils of rectangular cross section, Equal coils of rectangular cross section, Square cross section.

Current balance, of Ayrton and Jones, 104, 106; of National Physical Laboratory, 107.

Current sheets, 76,97, I I 9 .

Cylindrical wire. See Straight cylindrical wire.

Cylindrical coils. See Solenoids, Coaxial solenoids.

Cylindrical conductors. See Linear conductors.

Decrease of inductance with the frequency: General considerations, 172 ; of straight cylindrical wires, $173,174,177,179,183-185$; of two parallel cylindrical wires, 180 , $18 \mathrm{I}, 185,186$; of a circular ring, $18 \mathrm{I}, 182,186,187$.

Differential coefficients, 34,39 .

Dimensions of equivalent current sheet 76,97, II9.

Disk in Lorenz apparatus. See Lorenz apparatus.

Distribution of current. See Correction for unequal, High frequency formulas, Increase of resistance, Decrease of inductance.

Dynamometers: Gray, 60, 86; Ayrton and Jones, 104, 106.

\section{E}

E $d d y$ currents. See High frequency formulas, Decrease of inductance, Increase of resistance.

Ellipse. See Elliptical area.

Elliptical area, geometric mean distance of, 167.

Elliptic integrals. See also Incomplete elliptic integrals, mutual inductance formulas involving $6,7,9,64,68,71,98,99$, 100; inductance of solenoids, II8; series expansions for complete, 8,9 ; tables of, as functions of $\tan \gamma, 193$; Legendre's Tables, 202-2I 2 .

End correction: For mutual inductance of equal concentric solenoids, 55 ; for selfinductance of solenoids, II9-121, $130-132,223-225$.

End effect. See End correction. 
Equal circles, mutual inductance of $\mathrm{I} 4,28,30, \mathrm{I} 8$.

Equal coils of rectangular cross section, mutual inductance of, 33, 39, 40, 44, 45, 47, $49-5 I$.

Equal parallel rectangles. See Parallel rectangles.

Equal squares. See Squares, Geometric mean distance.

Equal solenoids, mutual inductance of, 69-7I, 94-97.

Equal radii. See Equal circles, Equal coils of rectangular cross section, Equal solenoids, Solenoids of equal radii.

Equal cross section. See Equal coils of rectangular cross section.

Equivalent circles. See Equivalent filaments.

Equivalent breadth. See Equivalent filaments.

Equivalent radius. See Equivalent filaments.

Equivalent length. See Dimensions of equivalent current sheet.

Equivalent filaments, 38, 39, 47, 48 .

Frrors in Rowland's and Rayleigh's formulas, 36, 37.

Exact formulas. See Absolute formulas.

Examples illustrating the formulas: For mutual inductance of coaxial circles, 20-32; mutual inductance of coaxial coils of rectangular cross section, 44-52; mutual inductance of coaxial solenoids, 77-98; mutual inductance of circle and solenoid, I03-IIO; self-inductance of circular ring, II4-II5; self-inductance of solenoids, $126-135$; self-inductance of coils of rectangular cross section, $142-150$; self and mutual inductance of linear conductors, 159-166; inductance and resistance with high frequency, $183-187$.

Extension of Maxwell's series formula for circles, I4, 30; Maxwell's formula for equal, concentric solenoids, $53,77-80$; Ròiti's formula for coaxial solenoids, $57-59$, 80-86; Jones's formula for circle and solenoid, IOI, I02, I03-106, I08, I09; Rayleigh's and Nivens's formula for the inductance of solenoids, II7, 129; Russell's formulas for $W, Y$, and $Z, 176$.

Filaments. See Equivalent filaments.

Force of attraction of coils. See Dynamometers, Current balances.

Formulas. See Absolute formulas, Approximate formulas, Correction formulas, Interpolation formulas.

Frequency. See High frequency formulas.

Fröhlich, inductance of toroidal coil, I25.

Functions. See Bessel functions, $\mathbf{p}$ function, $q$ series, Ber and bei functions, $K e r$. and $k e i$ functions, tables, coefficients, $W, Y$, and $Z$.

\section{$\mathrm{G}$}

General term: In Wallis's formulas for $F$ and $E, 9$; in formulas (5) and (6), 9, ro; in Havelock's formula for coaxial solenoids, 56; in Gray's and Searle and Airey's formulas, 63; in Russell's formula for coaxial solenoid, 68; in Lorenz's formula for circle and solenoid, 99; in the Webster-Havelock formula, I2I; in Russell's formulas for $W, Y$, and $Z$, i 76 .

Geometric mean distance, 42, 52, 166-I70.

Glazebrook, 34 .

Gray, mutual inductance of coaxial solenoids, 59, 60, 86-89; dynamometer, 6o, 86; geometric mean distance formulas, I7o.

\section{H}

Harmonics. See Zona1 harmonics.

Hazelock, mutual inductance of coaxial circles, $15,16,27,29,30$; mutual inductance of coaxial solenoids, $55,56,72,78$; mutual inductance of short secondary on long primary, 68; self-inductance of solenoids (see Webster).

Heaviside, equal coaxial solenoids, 55, 78; high frequency formulas, 173.

Hicks, inductance of a ring, II3.

High frequency formulas, III, I72-I87. 
Himstedt, mutual inductance of solenoids, 72 .

Hollow tube. See Tube.

Hypergeometric Series, I7, 56, Table XVII.

\section{I}

I1lustration of formulas. See Examples for illustrating the formulas.

Incomplete elliptic integrals, 64, 67, Ioo.

Increase of resistance with the frequency, I72-I74, I77, I78-I83, 183-187, Table XXII.

Infinite solenoid, inductance of, II6.

Insulation of wires, correction for, $138,139,140-142$.

Integrals. See Elliptic integrals, Incomplete elliptic integrals.

Interpolation: In Tables XV and XVI, I2; in Table XXII, I77; formula for, 214.

Invariant. See Absolute invariant.

\section{$\mathrm{J}$}

Jacobi, $q$ series, II, I2, 65-67, I20, I2I; Theta functions, 66.

Jones, mutual inductance of circle and solenoid, 99, 102, ro6; see also Ayrton, Campbell.

Joubert. See Mascart.

\section{K}

Kelvin, resistance and inductance of straight wires at high frequency, I73, 174; ber and $b e i$ functions, I75.

Ker and Kei functions, I75.

Kirchhoff, inductance of a ring, IIO, II 4 ; inductance of a solenoid, II8; summation formula for the inductance of a solenoid, I23; inductance of a square, I54; formula for mutual inductance of coaxial coils, 73 .

\section{L}

Landen's transformation, 7 .

Layers, coils of several. See Solenoids.

Legendre's tables, 6, 8, I0, 20, 44, 10o, Tables XII and XIII.

Limiting formulas for resistance and inductance of straight wires with the frequency, I77.

Line, geometric mean distance of, I67; geometrical mean distance of two lines, I68, I69, I70; arithmetical mean distance of, I7I; arithmetical mean square distance of, ITI.

Linear conductors, self and mutual inductance of, I50-159, 159-166; mutual inductance of two conductors in the same straight line, I52, I6I.

Logarithins. See Natural logarithms.

Long coils, mutual inductance, choice of formulas, 73-77; self-inductance of, II6, II 7, I 2O, I29, I3O-I33.

Lorenz, apparatus, 34, 103, 107; mutual inductance of circle and solenoid, 9S; inductance of solenoids, II 7 , II $8,129,130,132,133$.

Lyle, mutual inductance of coils of rectangular cross section, $38,47,48$.

\section{II}

Martens, mutual inductance of rectangles, 156 .

Mascart, 55.

Wathy, corrected formula of, for coaxial circles, $I 7,3 I$; simple special formula derived from this, $18,32$.

Maxwell, mutual inductance of coaxial circles, 6-8, I3, 20, 2I, 28, Table I; mutual inductance of equal coaxial, concentric solenoids, $53,77,79,80$; inductance of a ring, IIO, III, II4, II5; inductance of coil of rectangular cross section, I35, I 36 , 142,143 ; correction for distribution of current in round wires, I4O. 
McGill University, Lorenz apparatus of, 103.

Method of obtaining dimension of coils. See Dimensions of equivalent current sheets.

Minchin, inductance of ring, II 3 .

Misprints in authorities quoted, $34,55,64$, I00, I03, I70.

Modified Radius. See Equivalent filaments.

Modulus. See Elliptic integrals, Incomplete integrals, Complementary modulus.

Multiple conductors, inductance of, 159,165 .

Mutual inductance. See detailed headings, such as Coaxial circles, Coaxial solenoids, etc.

Mutual inductance by means of self-inductance formulas, 7 I, 95, 96 .

\section{$\mathrm{N}$}

Nagaoka, mutual inductance of coaxial circles, II, I2, 26, 27, Table XV and XVI; mutual inductance of coaxial solenoids $64-67,73,89,91-93$; inductance of solenoids, II9-I2I, $130-132$.

Nasmyth, 87.

National Physical Laboratory, current balance of, 107.

Natural logarithms of numbers from I to Ioo, Table XI.

Neumann, inductance of straight cylindrical wire, $15 \mathrm{I}, 159$; mutual inductance of parallel rectangles, $156, I 64$.

Nicholson, inductance and resistance of parallel wires at high frequency, I8o.

Niven. See Rayleigh.

Noninductive Shunts, inductance of, $15^{8}$.

0

Ohm, determination of, 34 ; see also Lorenz apparatus.

Olshausen, absolute formulas for mutual inductance of coaxial coils, 73 .

\section{$P$}

p function of Weierstrass, I7, 65 .

Parallel bars. See Rectangular bars.

Parallel circles. See Coaxial circles.

Parallel lines. See Lines.

Parallel rectangles. See Rectangles.

Parallel squares. See Squares.

Parallel wires, mutual inductance of, 151,160 ; see also Return circuit.

Parameter, in Olshausen's formula, 73.

Permeability. See High frequency formulas, Linear conductors.

Perry, inductance of coil of rectangular cross section, I36, I43.

Pitch of winding, 76, 97, I I 9 .

Plane, coaxial circles in the same, I4, I5, IS.

$q$ Series of Jacobi, II, 65-67.

Quadratures, formula of, $34,35,45,46,90$.

$\mathbf{R}$

Rayleigh (a1so Rayleigh and Niven), formula of quadratures, $34,35,45,46$, 9o; inductance of circular ring, III, II4, II5; inductance of solenoids, II6, I26-129; inductance and resistance of straight wires at high frequency, I 73,177 .

Rectangles, geometric mean distance of, see Rectangular area; inductance of, I54, I55, I63, I64; mutual inductance of parallel rectangles, I55, I64.

Rectangular areas, geometric mean distance of, 167,168 ; geometrical mean distance of two, I 70 . 
Rectangular bars, inductance of, I52, I53, I62; mutual inductance of, I53, I54.

Rectangular cross section. See Coaxial coils of rectangular cross section, Equal coils of rectangular cross section.

Reduction from current sheet to winding of round wires, I22, I28, Tables VII and VIII.

Resistance. See Increase of resistance with the frequency.

Return Circuit, inductance of parallel wire, I5I, I52, I6 T; same at high frequency, I80, I8I, 185,186 ; inductance with rectangular cross section, I54, I62.

Ring, inductance of circular solid, IIO-II2, II4; inductance with elliptical cross section, II3; inductance and resistance with high frequency, I8I, I82, 186.

Ròiti, Mutual inductance of coaxial solenoids, $57,58,80-84,87$.

Rosa, extension of Maxwell's formula for coaxial coils, I 4,30 ; mutual inductance of coils of rectangular cross section, $39,49,50 ;$ Rosa-Weinstein formula for coaxial coils, 40, 4I, 5I, 69-7I, 94-97; extension of Searle and Airey's formula 6I-63, 8286, 94; mutual inductance of circle and solenoid, IOI, IO2, I03, 105,109 ; correction for inductance of toroidal coil, I25, I34, I35; correction of current sheet formulas for winding of round wires, I22, $128-130$, Tables VII and VIII; correction for distribution of current in coil of rectangular cross section, I38, I39, I4I, 144150, Tables IX and X; geometric mean distance, I68-I70; arithmetical mean distance and arithmetical mean square distance, I7I, I72.

Rowland, mutual inductance of coaxial coils, $33,34,44,45$.

Russell, mutual inductance of coaxial solenoids, $67,68,69,83$; self-inductance of solenoids, I2I; formulas for the functions $W, Y$, and $Z, 175,176$; inductance and resistance of concentric main, I79.

\section{$\mathrm{S}$}

Savidge, tables of functions for high frequency calculations, I75, I76.

Searle and Airey, mutual inductance of coaxial solenoids, 61-63, 82-86, 94 .

Self-inductance, mutual inductance by means of, $41,52,71,95,96$; see also detailed headings as Solenoids, Circular coils of rectangular cross sections, etc.

Series formulas for $F$ and $E, 8,9,22$, see Hypergeometric Series, $W, Y$, and $Z$ functions, and other detailed headings.

Short coils, inductance of short solenoids, I16, I20, $126,128,130$.

Short secondary on long primary, mutual inductance of, 68, 69, 93, 94 .

Shunts. See Noninductive shunts.

Simple formula for certain coaxial circles, I8, 32 .

Single layer coils. See Solenoids, Circle and coaxial solenoids.

Solenoids, inductance of infinite, I16; inductance of, II6-I26, 126-135; inductance of solenoid of more than one layer I3S-I4O, I5O; see also Coaxial solenoids.

Solid Ring. See Ring.

Squares, inductance of, I54, I62, I64; mutual inductance of parallel, I55, I56, I64; geometric mean distance of, I67.

Square cross section, mutual inductance of coaxial coils of, 39, 4I, 47, 49.

Stefan, mutual inductance of coaxial coils, 40; inductance of circular coil of rectangular cross section, $137,144^{-147}, 149$; correction for distribution of current in round wires, I4I.

Stirling's constants, I7, I9.

Straight cylindrical wire, inductance of, I50, I51, 159, I60; inductance and resistance of, at high frequency, I73-I79, $183-185$, Tables XXII, XXIII.

Strasser, inductance of single layer coil, I23, I24, I34, I47, Table V.

Strip. See Tape.

Subdivision, calculation of inductance by, 43, 74, 88-90.

Summation formula for inductance of solenoid, I23, I27.

Sumec, inductance of rectangle of rectangular cross section, I55.

\section{$\mathrm{T}$}

Tables of Constants, I89-23o. For detailed summary see Table of contents.

Tape, winding of II9; self and mutual inductance of, $156,157,165$; geometriç mean distance of, 168, 169. 
Taylor's theorem, 33 .

Terezawa, inductance of ring, footnote III.

Theta functions, 66.

Thick current sheet, I38, I39.

Thin strip. See Tape.

Thin-walled Tube. See Tube.

Thomson, J. J., inductance of ring of elliptical section, II3.

Toroidal coil, inductance of, I24, I25, 134 .

Tube, inductance of hollow, tubular ring, II2, II3, II5; straight tube, see High frequency formulas.

$\mathrm{U}$

Unequal circles. See Coaxial circles.

Unequal cross section, choice of formulas for mutual inductance of coils of, 43 .

Uniform magnetic field, inductance of ring rotating in, II3, II4.

Uniform distribution of current in cross section, I40, I4I, 147-149; see also High frequency formulas.

Uniform winding. See Dimensions of equivalent current sheets.

\section{$\mathrm{W}$}

$\mathbf{W}, \mathbf{Y}$, and $\mathbf{Z}$ functions, definitions of, $\mathrm{I74}$; expansions for, $\mathrm{I} 76, \mathbf{I} 77$; see also Table XXII.

Wallis, series expansions for elliptic integrals, 9.

Webster, inductance of a solenoid, I2I, 132 .

Weierstrass, $\mathbf{p}$ functions, 17,65 .

Weinstein, mutual inductance of coaxial circles, $\overline{7}, 25 ;$ mutual inductance of coaxial coils, 40, 4I, 5I, 7x, 96; inductance of coil of rectangular cross section, I37, 143. Wiedemann, mutual inductance of circles, footnote, 13.

Wien, inductance of ring, II I, II2; ring rotating in uniform magnetic field, II3, II4.

Y

$\mathbf{Y}$ function. See $W, Y$, and $Z$ functions.

\section{Z}

$\mathrm{Z}$ function. See $W, Y$, and $Z$ functions.

Zonal harmonics, 59 .

$2{ }^{2} 674^{\circ}-\mathrm{I} 2-\mathrm{I} 6$ 